\title{
AFFINE FLAG VARIETIES AND QUANTUM SYMMETRIC PAIRS
}

\author{
ZHAOBING FAN, CHUN-JU LAI, YIQIANG LI, LI LUO, AND WEIQIANG WANG
}

\begin{abstract}
The quantum groups of finite and affine type $A$ admit geometric realizations in terms of partial flag varieties of finite and affine type $A$. Recently, the quantum group associated to partial flag varieties of finite type $B / C$ is shown to be a coideal subalgebra of the quantum group of finite type $A$. In this paper we study the structures of Schur algebras and Lusztig algebras associated to (four variants of) partial flag varieties of affine type $C$. We show that the quantum groups arising from Lusztig algebras and Schur algebras via stabilization procedures are (idempotented) coideal subalgebras of quantum groups of affine $\mathfrak{s l}$ and $\mathfrak{g l}$ types, respectively. In this way, we provide geometric realizations of eight quantum symmetric pairs of affine types. We construct monomial and canonical bases of all these quantum (Schur, Lusztig, and coideal) algebras. For the idempotented coideal algebras of affine $\mathfrak{s l}$ type, we establish the positivity properties of the canonical basis with respect to multiplication, comultiplication and a bilinear pairing. In particular, we obtain a new and geometric construction of the idempotented quantum affine $\mathfrak{g l}$ and its canonical basis.
\end{abstract}

\section{Contents}

Chapter 1. Introduction

1.1. Background

1.2. The goal: affine type $C$

1.3. An overview

1.4. The organization

Chapter 2. Constructions in affine type $A$

2.1. Lattice presentation of affine flag varieties of type $A \quad 16$

2.2. Monomial basis for quantum affine $\mathfrak{s l}_{n}$

2.3. Algebras $\mathbf{U}_{n}$ and $\dot{\mathbf{U}}_{n}$

\begin{tabular}{ll|l|} 
Chapter 3. & Lattice presentation of affine flag varieties of type $C$ & 23 \\
\hline
\end{tabular}

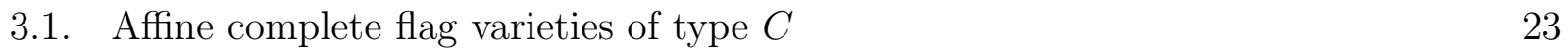

3.2. Affine partial flag varieties of type $C \quad 25$

3.3. Local property at $L_{0} \quad 26$

Chapter 4. Multiplication formulas for Chevalley generators $\quad 28$

4.1. Some dimension computation $\quad 28$

4.2. Standard and canonical bases of Schur algebras 29

4.3. Some multiplication formulas 32

2010 Mathematics Subject Classification. 17B37, 20G25, 14F43.

Key words and phrases. Affine flag variety, affine quantum symmetric pair, canonical basis. 
4.4. The leading term 36

Chapter 5. Coideal algebra type structures of Schur algebras and Lusztig algebras 39

5.1. The Lusztig algebra $\mathbf{U}_{n, d}^{\mathfrak{c}} \quad 39$

5.2. A raw comultiplication 4

5.3. The comultiplication $\Delta^{\mathfrak{c}} \quad 43$

5.4. Monomial and canonical bases of $\mathbf{U}_{n, d}^{\mathfrak{c}} \quad 47$

Part 2. Lusztig algebras and coideal subalgebras of $\mathbf{U}\left(\hat{\mathfrak{s l}}_{n}\right) \quad 49$

Chapter 6. Realization of the idempotented coideal subalgebra $\dot{\mathbf{U}}_{n}^{\mathfrak{c}}$ of $\mathbf{U}\left(\hat{\mathfrak{s l}}_{n}\right) \quad 50$

6.1. The coideal subalgebra $\mathbf{U}_{n}^{\mathfrak{c}}$ of $\mathbf{U}_{n} \quad 50$

6.2. The algebra $\dot{\mathbf{U}}_{n}^{\mathfrak{c}}$ and its monomial basis 52

6.3. Bilinear form on $\dot{\mathbf{U}}_{n}^{\mathfrak{c}} \quad 55$

6.4. The canonical basis of $\dot{\mathbf{U}}_{n}^{\mathfrak{c}}$ and positivity 56

6.5. Another presentation of the algebra $\dot{\mathbf{U}}_{n}^{\mathfrak{c}} \quad 57$

\begin{tabular}{lll|} 
Chapter 7. A second coideal subalgebra of quantum affine $\mathfrak{s l}_{\mathfrak{n}}$ & 59 \\
\hline
\end{tabular}

7.1. The Schur algebras of type $\imath \quad 59$

7.2. The comultiplication $\quad 60$

7.3. The monomial basis of $\mathbf{U}_{\mathfrak{n}, d}^{\jmath \imath} \quad 63$

7.4. The coideal subalgebra of type $\imath \quad 65$

\begin{tabular}{lll|} 
Chapter 8. More variants of coideal subalgebras of quantum affine $\mathfrak{s l}_{n}$ & 68 \\
\hline
\end{tabular}

8.1. The Schur algebras of type $\vartheta$

8.2. Comultiplication and transfer map of type $y \quad 69$

8.3. Quantum symmetric pair $\left(\mathbf{U}\left(\widehat{\mathfrak{s l}}_{\mathfrak{n}}\right), \mathbf{U}_{\mathfrak{n}}^{\imath \jmath}\right)$ and canonical basis on $\dot{\mathbf{U}}_{\mathfrak{n}}$

8.4. The Schur algebras of type $\imath \imath$

8.5. Realization of a new coideal subalgebra $\mathbf{U}_{\eta}^{\imath 2} 74$

$\begin{array}{lll}\text { Part 3. Schur algebras and coideal subalgebras of } \mathbf{U}\left(\hat{\mathfrak{g l}}_{n}\right) & 77\end{array}$

Chapter 9. The stabilization algebra $\dot{\mathbf{K}}_{n}^{\mathfrak{c}}$ arising from Schur algebras $\quad 78$

9.1. Monomial bases for Schur algebras $\quad 78$

9.2. Stabilization of the Schur algebras $\quad 82$

9.3. Comultiplication and stabilization 85

9.4. The algebra $\dot{\mathbf{K}}_{n}^{\mathfrak{c}}$ and its stably canonical basis 86

9.5. The algebra $\dot{\mathbf{K}}_{n}$ of affine type $A$ and its comultiplication $\quad 88$

9.6. The comultiplication on $\dot{\mathbf{K}}_{n}^{\mathfrak{c}} \quad 90$

9.7. A homomorphism from $\dot{\mathbf{K}}_{n}^{\mathfrak{c}}$ to $\mathbf{S}_{n, d}^{\mathfrak{c}}$

9.8. The algebra $\dot{\mathbf{K}}_{n}^{\mathfrak{c}}$ as a subquotient of $\dot{\mathbf{K}}_{\breve{n}}^{\mathfrak{c}}$

Chapter 10. Stabilization algebras arising from other Schur algebras $\quad 94$

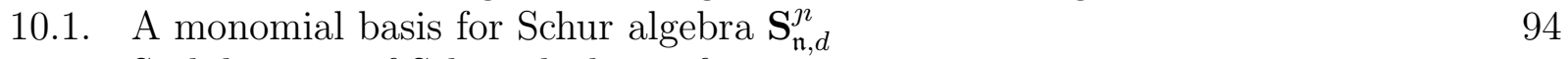

10.2. Stabilization of Schur algebras of type $\imath \imath$

\begin{tabular}{lll} 
10.3. The stabilization algebra $\dot{\mathbf{K}}_{n}^{\jmath \imath}$ & 96 \\
\hline
\end{tabular} 
10.4. Stabilization algebra of type $\vartheta$

10.5. Stabilization algebra of type $\imath \imath$

Appendix A. Constructions in finite type $C$

101

A.1. Multiplication formulas

A.2. Isomorphisms between type $\mathrm{C}$ and type $\mathrm{B}$

A.3. The comultiplication

Appendix. List of Notations 


\section{CHAPTER 1}

\section{Introduction}

\subsection{BACKGROUND}

1.1.1. Iwahori Iw64 provided a geometric realization of Iwahori-Hecke algebras $\mathbf{H}_{W}^{\mathrm{fin}}$ as convolution algebras on pairs of (finite type) complete flags over a finite field. IwahoriMatsumoto IM65 have subsequently realized the affine Hecke algebras using pairs of complete flags of affine (or $p$-adic) type over a local field. These works are foundational for geometric representation theory.

The Drinfeld-Jimbo quantum groups [Dr86, Jim86] have played important roles in many areas of mathematics. Beilinson, Lusztig and MacPherson [BLM90] provided a geometric realization of quantum Schur algebras and the quantum group $\mathbf{U}\left(\mathfrak{g l}_{n}\right)$ of finite type $A$. The BLM construction utilizes the $n$-step flag varieties in an ambient space of dimension $d$, and the convolution algebra on pairs of $n$-step flags can be identified with what became known as quantum Schur algebra $\mathbf{S}_{n, d}^{\text {fin }}$; this can be viewed as a generalization of Iwahori's construction of Hecke algebras in finite type $A$.

Beilinson, Lusztig and MacPherson [BLM90] further established multiplication formulas in $\mathbf{S}_{n, d}^{\mathrm{fin}}$ with divided powers of Chevalley generators, which allows them to observe some remarkable stabilization phenomenon as $d \rightarrow \infty$. A suitable limit construction gives rise to the idempotented quantum group $\dot{\mathbf{U}}\left(\mathfrak{g l}_{n}\right)$ and its (stably) canonical basis. The construction is easily modified further to produce variants such as $\mathbf{U}\left(\mathfrak{g l}_{n}\right), \mathbf{U}\left(\mathfrak{s l}_{n}\right)$, and the idempotented form $\dot{\mathbf{U}}\left(\mathfrak{s l}_{n}\right)$. The idempotented form $\dot{\mathbf{U}}\left(\mathfrak{s l}_{n}\right)$ also has a canonical basis (cf. [Lu93, [K94]), in analog with the Kazhdan-Lusztig bases for Iwahori-Hecke algebras [KL79].

1.1.2. Independently and around the same time, Dipper and James [DJ89, DJ91] introduced the quantum Schur algebra as the endomorphism algebra of a sum of permutation modules of the finite type $A$ Hecke algebra. A version of quantum $G L_{n}$ [DD91] also fits well in this framework. The identification between algebraic and geometric definitions of the quantum Schur algebra follows as either version of quantum Schur algebra forms the centralizer of the Hecke algebra action on the same tensor space [DJ91, GL92]; see [Du92]. Moreover, there are natural surjective homomorphisms

$$
\dot{\mathbf{U}}\left(\mathfrak{g l}_{n}\right) \rightarrow \mathbf{S}_{n, d}^{\text {fin }}, \quad \dot{\mathbf{U}}\left(\mathfrak{s l}_{n}\right) \rightarrow \mathbf{S}_{n, d}^{\text {fin }} .
$$

In this way, we have obtained $q$-Schur duality or Schur-Jimbo duality [Jim86].

1.1.3. There have been some generalizations of the BLM-type construction using the $n$ step (partial) flag varieties of affine type $A$ earlier on; see Ginzburg-Vasserot GV93 and Lusztig Lu99, Lu00, (also cf. [VV99, Mc12, P09, GRV93, for further developments); see also [CP96] for an affine version of Schur-Jimbo duality. We shall refer to the convolution algebra arising this way as affine quantum Schur algebra, denoted by $\mathbf{S}_{n, d}$. However, there is 
a major difference between affine and finite type $A$, which was first made clear by Lusztig. He showed that a natural homomorphism from the quantum affine $\mathfrak{s l}_{n}$ of level zero to the affine quantum Schur algebra,

$$
\dot{\mathbf{U}}\left(\hat{\mathfrak{s l}}_{n}\right) \longrightarrow \mathbf{S}_{n, d}
$$

is no longer surjective (the image of this map is denoted by $\mathbf{U}_{n, d}$ and called Lusztig algebra in this paper). Alternatively, one could characterize $\mathbf{U}_{n, d}$ as the proper subalgebra of $\mathbf{S}_{n, d}$ generated by the Chevalley generators.

There has been a new (algebraic) approach recently developed by [DF13, DF14] (see also [G99]) which allows one to construct a larger algebra $\dot{\mathbf{U}}\left(\widehat{\mathfrak{g l}}_{n}\right)$ (called the idempotented quantum affine $\mathfrak{g l}_{n}$ in this paper; also known as the quantum loop algebra of $\mathfrak{g l}_{n}$ ), from BLM-type stabilization of the affine Schur algebras $\mathbf{S}_{n, d}$.

1.1.4. Since the constructions of Iwahori and Iwahori-Matsumoto are valid for flag varieties of any finite and affine type, it is a natural question since the work of [BLM90] in 1990 to ask for generalization of the above type $A$ constructions to other, say classical, types. The progress in this direction has been made only in recent years. Motivated by [BW13, Bao, Kujawa, and two of the authors [BKLW14, BLW14] provided a geometric construction of Schur-type algebras $\mathbf{i} \mathbf{S}_{n, d}^{\text {fin }}$ (denoted therein by $\mathbf{S}^{\jmath}$ for $n$ odd and $\mathbf{S}^{\imath}$ for $n$ even) in terms of $n$-step flag varieties of type $B_{d}$ (or $C_{d}$ ).

The authors of [BKLW14, BLW14] further established multiplication formulas in the Schur algebras $\mathbf{i} \mathbf{S}_{n, d}^{\text {fin }}$ with divided powers of Chevalley generators, which again enjoy some remarkable stabilization properties as $d \mapsto \infty$. They showed the quantum algebra arising from the stabilization procedure is a coideal subalgebra $\mathbf{i U}\left(\mathfrak{g l}_{n}\right)$ of $\mathbf{U}\left(\mathfrak{g l}_{n}\right)$ (this coideal subalgebra was denoted in loc. cit. as $\mathbf{U}^{\jmath}$ for $n$ odd and $\mathbf{U}^{\imath}$ for $n$ even); the pair $\left(\mathbf{U}\left(\mathfrak{g l}_{n}\right), \mathbf{i} \mathbf{U}\left(\mathfrak{g l}_{n}\right)\right)$ forms a so-called quantum symmetric pair, which we shall explain below.

1.1.5. Let $\mathfrak{g}$ be a symmetrizable Kac-Moody algebra over $\mathbb{C}$ and $\mathbf{U}(\mathfrak{g})$ be the quantized enveloping algebra of $\mathfrak{g}$. Let $\imath$ be a Lie algebra involution on $\mathfrak{g}$ of the second kind (cf., e.g., [Ko14, §2]) and let $\mathfrak{g}^{\imath}$ be the subalgebra of $\imath$-invariants in $\mathfrak{g}$. (For simple Lie algebras $\mathfrak{g}$ of finite type, the classification of $\mathfrak{g}^{2}$ corresponds to the classification of real simple Lie algebras, cf. [OV].) The quantum analogue $\mathbf{i U}(\mathfrak{g})$ of the enveloping algebra $U\left(\mathfrak{g}^{\imath}\right)$ is not a Hopf algebra, but it is a coideal subalgebra of $\mathbf{U}(\mathfrak{g})$ in the sense that the comultiplication $\Delta$ on $\mathbf{U}(\mathfrak{g})$ satisfies

$$
\Delta: \mathrm{iU}(\mathfrak{g}) \longrightarrow \mathrm{iU}(\mathfrak{g}) \otimes \mathbf{U}(\mathfrak{g}) .
$$

By [Le02, Theorem 7.5] and [Ko14, Theorem 10.8], $\mathbf{i U}(\mathfrak{g})$ specializes to $U\left(\mathfrak{g}^{2}\right)$ at $q=1$. The pair $(\mathbf{U}(\mathfrak{g}), \mathbf{i U}(\mathfrak{g}))$ is called a quantum symmetric pair. The algebra $\mathbf{i U}(\mathfrak{g})$ admits a Serre-type presentations which is nevertheless more complicated.

The theory of quantum symmetric pairs was systematically developed by Letzter [Le99, Le02] for finite type (also see [N96] for some early examples). It was subsequently generalized by Kolb [Ko14] to the Kac-Moody setting, and one can find in loc. cit. an informative introduction for the background and extensive references on quantum symmetric pairs.

There are recent and ongoing developments for general coideal algebras $\mathbf{i U}(\mathfrak{g})$ in a way strikingly parallel to the well-known constructions associated to the Drinfeld-Jimbo quantum groups, such as connections to canonical bases, categorification, geometry, and solutions to generalized Yang-Baxter equations (known as reflection equations); cf., e.g., [BK15, BW16] 
for samples. To distinguish from many other different coideal subalgebras in the literature, we shall refer to the coideal subalgebras appearing in quantum symmetric pairs as quantum groups, where $\imath$ stands for involution or isotropic.

1.1.6. An $\left(\mathbf{i U}\left(\mathfrak{g l}_{n}\right), \mathbf{H}_{C_{d}}^{\text {fin }}\right)$-duality (termed as iSchur duality) was discovered algebraically and categorically in BW13] as a crucial ingredient for a new approach to Kazhdan-Lusztig theory of classical type. A new canonical basis (called $\imath$ canonical basis) was constructed in [BW13] for various tensor product modules of $\mathbf{i U}\left(\mathfrak{g l}_{n}\right)$. In the special case of the tensor product of the natural representation of $\mathbf{i U}\left(\mathfrak{g l}_{n}\right)$, the $\imath$ canonical basis was shown to coincide with the Kazhdan-Lusztig basis of type B, and thus it provides a new approach to the irreducible and tilting character problems in the BGG category $\mathcal{O}$. (Similarly, the type D KL basis is identified with $\imath$ canonical basis with a different choice of parameter [Bao16], and thus $\imath$ canonical bases provide a new uniform approach for KL theory of classical type.) We refer to loc. cit. for further applications of $\imath$ canonical basis to KL theory of ortho-symplectic Lie superalgebras.

1.1.7. The iSchur duality has been subsequently realized in [BKLW14] by using mixed pairs of $n$-step flags and complete flags of type B/C. The $\imath$ canonical basis for the idempotented form $\mathbf{i} \dot{\mathbf{U}}\left(\mathfrak{g l}_{n}\right)$ was first constructed in loc. cit.. It has been shown in [FL14 that coideal like algebras together with their $\imath$ canonical bases arise from partial flag varieties of type $D$. There has been a further geometric realization in [LW15] of the idempotented coideal subalgebra $\mathbf{i} \dot{\mathbf{U}}\left(\mathfrak{s l}_{n}\right)$ of $\mathbf{U}\left(\mathfrak{s l}_{n}\right)$ and its $\imath$ canonical basis.

For canonical bases, there is a major difference between $\dot{\mathbf{U}}\left(\mathfrak{g l}_{n}\right)$ and $\dot{\mathbf{U}}\left(\mathfrak{s l}_{n}\right)$, or between idempotented coideal subalgebras of $\mathfrak{g l}$ and $\mathfrak{s l}$ type: the canonical basis of $\dot{\mathbf{U}}\left(\mathfrak{s l}_{n}\right)$ admits remarkable positivity properties with respect to multiplication and a bilinear pairing [SV00, Mc12, LW15] and so does the canonical basis of $\mathbf{i} \dot{U}\left(\mathfrak{s l}_{n}\right)$ [LW15]. It is recently shown in [FL15] that the canonical bases of idempotented quantum (affine) $\mathfrak{s l}_{n}$ and idempotented coideal algebra $\mathbf{i} \dot{\mathbf{U}}\left(\mathfrak{s l}_{n}\right)$ admit positivity property with respect to the comultiplication. In contrast, the canonical bases of $\dot{\mathbf{U}}\left(\mathfrak{g l}_{n}\right)$ and of $\mathbf{i} \dot{\mathbf{U}}\left(\mathfrak{g l}_{n}\right)$ both fail to exhibit a positivity property with respect to multiplication; see [LW15].

\subsection{The GOAL: AfFine TYPe $C$}

1.2.1. The goal of this paper is to initiate the study of the Schur algebras and quantum groups arising from partial flag varieties of classical affine type beyond type $A$, generalizing the constructions in finite type $B / C$ described in Section 1.1 .

In this paper, we focus on the affine type $C$. As we shall see, the affine type $C$ setting already provides a more challenging and much richer setting than the finite type $C$ and the affine type $A$. For each of the two type $A$ quantum affine algebras (of level zero) $\mathbf{U}\left(\widehat{\mathfrak{s l}}_{n}\right)$ and $\mathbf{U}\left(\hat{\mathfrak{g l}}_{n}\right)$, we shall provide geometric realizations of four different (idempotented) coideal

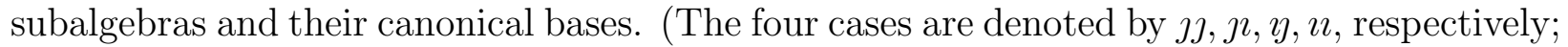
we also write $\mathfrak{c} \equiv \jmath$ J.) The corresponding four Dynkin diagrams with involutions are depicted in Figures 1, 2, 3] and 4, respectively, as follows, where $n=2 r+2,2 r+1,2 r+1$ and $2 r$, respectively. Therefore, in total we have provided a geometric realization of eight distinct quantum symmetric pairs of affine type. 
FIGURE 1. Dynkin diagram of type $A_{2 r+1}^{(1)}$ with involution of type $\jmath \jmath \equiv \mathfrak{c}$.

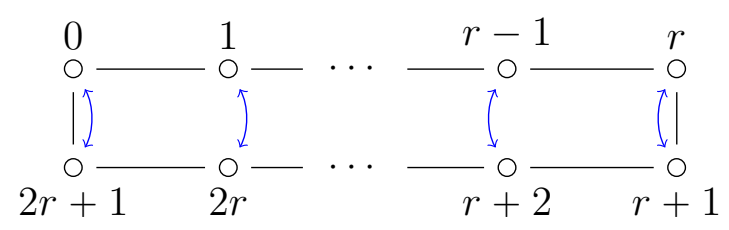

Figure 2. Dynkin diagram of type $A_{2 r}^{(1)}$ with involution of type $\jmath$.

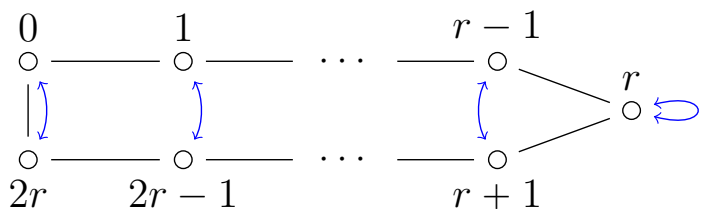

Figure 3. Dynkin diagram of type $A_{2 r}^{(1)}$ with involution of type $\imath$.

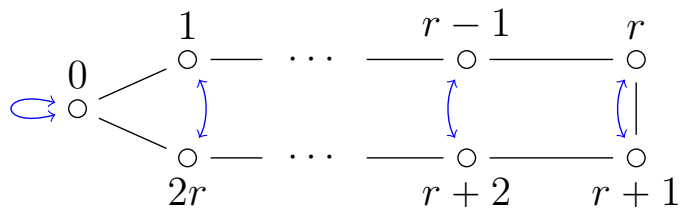

FiguRE 4. Dynkin diagram of type $A_{2 r-1}^{(1)}$ with involution of type $\imath$.

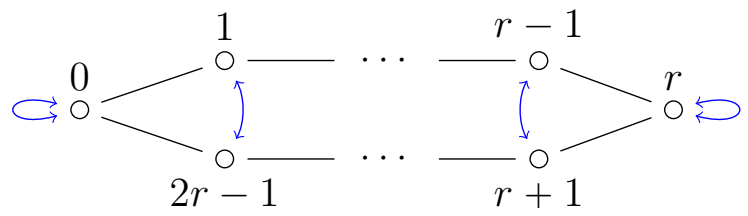

In summary, the quantum algebras behind the various kinds of flag varieties are listed in Table 1, as follows for comparison.

TABle 1. Comparison for various quantum algebras.

\begin{tabular}{|l|l|l|}
\hline Flag variety: & Complete flag & Partial flag \\
\hline \multirow{3}{*}{ of finite type } & \multirow{2}{*}{ Iwahori-Hecke algebra } & Type A: quantum $\mathfrak{g l}_{n}, \mathfrak{s l}_{n}$ \\
\cline { 3 - 3 } of affine type & Affine Iwahori-Hecke algebra $\begin{array}{r}\text { Type } / \text { coideal subalgebras } \\
\text { of quantum } \mathfrak{g l}_{n}, \mathfrak{s l}_{n}\end{array}$ \\
\cline { 3 - 3 } & $\begin{array}{r}\text { Type A: affine quantum } \mathfrak{g l}_{n}, \mathfrak{s l}_{n} \\
\text { of coideal subalgebras } \\
\text { of affine quantum } \mathfrak{g l}_{n}, \mathfrak{s l}_{n}\end{array}$ \\
\hline
\end{tabular}


1.2.2. To help the reader to follow and digest this long paper, we organize various chapters in three parts. Here is a brief summary.

- Part 1 contains the basic constructions of the affine Schur algebra $\mathbf{S}_{n, d}^{\mathfrak{c}}$ and its distinguished Lusztig subalgebra $\mathbf{U}_{n, d}^{\mathfrak{c}}$, as well as their $\imath \imath, \imath \jmath, \imath$-variants. Then we study in depth the multiplicative and coideal like comultiplicative structures of these algebras.

- In Part 2 we study the structures of the family of Lusztig algebras $\mathbf{U}_{n, d}^{\mathfrak{c}}$ (and their $\jmath, \imath \jmath, \imath$-siblings $)$, and show that they lead to coideal subalgebras $\mathbf{U}^{\mathfrak{c}}\left(\widehat{\mathfrak{s l}}_{n}\right)$ of $\mathbf{U}\left(\widehat{\mathfrak{s l}}_{n}\right)$. The corresponding idempotented forms $\dot{\mathbf{U}}^{\mathfrak{c}}\left(\widehat{\mathfrak{s l}}_{n}\right)$ (and their $\imath \imath, \imath, \imath$-siblings) are shown to admit canonical bases with positivity.

- Part 3 is focused on the study of the stabilization properties of the family of Schur

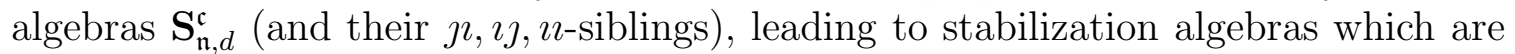
identified as idempotented coideal subalgebras $\mathbf{U}^{\mathfrak{c}}\left(\hat{\mathfrak{g l}}_{n}\right)$ of quantum affine $\mathfrak{g l}_{n}$; these stabilization algebras are shown to admit canonical bases (without positivity).

The following diagram is a brief road map of some main constructions (there are 4 distinct cases where $\mathfrak{c}$ can be replaced by $\jmath \jmath, \jmath \imath, \imath, \imath)$ :

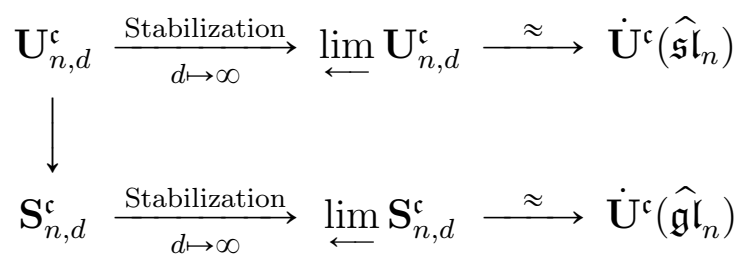

1.2.3. While the quantum algebras arising from partial flags of classical types (except type $A$ ) are not of Drinfeld-Jimbo quantum groups, they are meaningful and significant generalizations of the type $A$ quantum groups because of their geometric origin. There has been an intimately related category $\mathcal{O}$ interpretation and an application of canonical bases arising from quantum symmetric pairs of finite type [BW13] (also cf. [ES13, Bao16] for type D).

It is expected that the quantum symmetric pairs of affine type (and their categorifications) will play a fundamental role in modular representations of algebraic groups and quantum groups of classical type. We also expect a Langlands dual picture of the constructions of this paper, realizing the coideal algebras of affine type in terms of Steinberg-type varieties of finite type (cf. [CG97] for some earlier instances of such dual pictures).

\subsection{AN OVERVIEW}

\subsubsection{An overview of Part 1.}

1.3.1.1. Most of the geometric constructions in [BKLW14, BLW14] (and also [LW15, FL15]) in finite type $B / C$ were treated in two separate cases, depending on the parity of $n$, even though the statements are uniform. The results for $\mathbf{i} \mathbf{S}_{n, d}^{\text {fin }}$ and $\mathbf{i} \dot{\mathbf{U}}\left(\mathfrak{g l}_{n}\right)$ with $n$ odd are established first, and then the subtler even $n$ case is settled by relating to the odd $n$ case.

Before proceeding to the affine type, it is instructive for us to explain informally some of the main ideas of [BKLW14] (and [BLW14]). We shall fix an even positive integer $n$ and set $\mathfrak{n}=n+1$ (which is odd) in this section. We shall write $\mathbf{i} \mathbf{S}_{\mathfrak{n}, d}^{\text {fin }}=\mathbf{S}_{\mathfrak{n}, d}^{\jmath, \text { fin }}, \mathbf{i} \mathbf{U}\left(\mathfrak{g l}_{\mathfrak{n}}\right)=\mathbf{U}^{\jmath}\left(\mathfrak{g l}_{\mathfrak{n}}\right)$, $\mathbf{i} \mathbf{S}_{n, d}^{\text {fin }}=\mathbf{S}_{n, d}^{\imath, \text { fin }}, \mathbf{i} \mathbf{U}\left(\mathfrak{g l}_{n}\right)=\mathbf{U}^{\imath}\left(\mathfrak{g l}_{n}\right)$, and use similar notations for the idempotented forms. 
The Schur algebra $\mathbf{S}_{\mathfrak{n}, d}^{\jmath \text { fin }}$ is most naturally realized via pairs of $\mathfrak{n}$-step type $B$ flags. Even though the geometric realization for $\mathbf{S}_{n, d}^{2, \text { fin }}$ could naturally use $n$-step type $C$ flags, [BKLW14] instead chose to work with $\mathfrak{n}$-step type $B$ flags subject to a maximal isotropic condition on the middle subspaces of flags. This approach of using the type $B$ geometry alone allows one to relate the Schur algebras as well as the coideal algebras with indices $n, \mathfrak{n}$ of different parities.

The Dynkin diagram automorphism of type $\mathfrak{g l}_{\mathfrak{n}}$ has no fixed point as $\mathfrak{n}$ is odd, which is Figure (11) with vertices 0 and $2 r+1$ removed, while it has a fixed point for type $\mathfrak{g l}_{n}$, which is Figure (2) with vertices $0,2 r$ removed. Working with flags subject to maximal isotropic middle constraints can be loosely understood as giving rise to the Schur algebras and coideal algebras with a fixed point; the imbedding of such flags into a variety of flags without maximal isotropic constraints is a way of resolving such a fixed point, and this is how we succeeded in understanding $\mathbf{S}_{n, d}^{\imath, \text { fin }}$ (and respectively, $\dot{\mathbf{U}}^{\imath}\left(\mathfrak{g l}_{n}\right)$ ) through its relation to $\mathbf{S}_{\mathfrak{n}, d}^{\jmath, \text { fin }}$ (and respectively, $\dot{\mathbf{U}}^{\jmath}\left(\mathfrak{g l}_{\mathfrak{n}}\right)$ ).

As a preparation toward affine type $C$, we reformulate the main geometric constructions of [BKLW14, FL15] in the framework of finite type $C$ flags in Appendix A, expanding the outline in [BKLW14, §6]. Recall that $\mathbf{S}_{n, d}^{\imath, \text { fin }}$ can be realized using $n$-step type $C$ flags (note the middle subspace in such a flag is automatically maximal isotropic). To realize $\mathbf{S}_{\mathfrak{n}, d}^{\mathfrak{c}, \text { fin }}$ (recall $\mathfrak{n}=n+1$ ), we employ $\mathfrak{n}$-step type $C$ flags, and then identify an $n$-step flag as an $\mathfrak{n}$-step flag subject to a maximal isotropic condition on the middle subspace. Then all type $B$ constructions in [BKLW14, BLW14, FL15] can be repeated in such a finite type $C$ setting. (This might be regarded a manifestation of Langlands duality philosophy.)

1.3.1.2. Let us return to the affine cases. There is a lattice presentation of the complete and $n$-step flag varieties of affine type $A$ due to Lusztig; see Chapter 2. Such a lattice presentation can be adapted to affine type $C$, on which the symplectic loop group $\operatorname{Sp}_{F}(2 d)$ acts (where $F=k((\epsilon)))$; cf. Sage [Sa99] for complete flags and its variant for the $n$-step partial flag variety $\mathcal{X}_{n, d}^{\mathfrak{c}}$ which is formulated in this paper, for $n$ even.

However, for our purpose we need to define such a $\mathcal{X}_{n, d}^{\mathfrak{c}}$ in a somewhat delicate way, keeping in mind the lesson we learned from finite type $B / C$. That is, $\mathcal{X}_{n, d}^{\mathfrak{c}}$ is defined to avoid "maximal isotropic" constraints and (as shown later) it will give rise to Schur algebras associated to the affine Dynkin diagram automorphism without fixed points in Figure 1; the most obvious candidate of $n$-step flag variety of affine type $C$ will not do.

The orbits for the product $\mathcal{X}_{n, d}^{\mathfrak{c}} \times \mathcal{X}_{n, d}^{\mathfrak{c}}$ under the diagonal action of the group $\operatorname{Sp}_{F}(2 d)$ can be parameterized by the set $\Xi_{n, d}$ of $\mathbb{Z} \times \mathbb{Z}$-matrices with entries in $\mathbb{N}$ satisfying certain natural periodicity and centro-symmetry conditions. Denote by $\Xi_{n, d}^{a p}$ the set of aperiodic matrices in $\Xi_{n, d}$ (recall the notion of aperiodic matrix was introduced in [Lu99] in the affine type $A$ setting).

The Schur algebra $\mathbf{S}_{n, d}^{\mathfrak{c}}$ is by definition the (generic) convolution algebra of pairs of flags in $\mathcal{X}_{n, d}^{\mathfrak{c}}$. It admits a canonical basis (IC basis) which enjoys a positivity with respect to multiplication. We formulate a subalgebra $\mathbf{U}_{n, d}^{\mathfrak{c}}$ of $\mathbf{S}_{n, d}^{\mathfrak{c}}$ generated by the Chevalley generators. We caution that the Chevalley generators do not form a generating set for the algebra $\mathbf{S}_{n, d}^{\mathfrak{c}}$, that is, $\mathbf{U}_{n, d}^{\mathfrak{c}}$ is a proper subalgebra of $\mathbf{S}_{n, d}^{\mathfrak{c}}$ in general. Our first main result is the following. 
Theorem A (Theorem 5.4.3). The algebra $\mathbf{U}_{n, d}^{\mathfrak{c}}$ admits a monomial basis $\left\{\zeta_{A} \mid A \in \Xi_{n, d}^{a p}\right\}$ and a canonical basis $\left\{\{A\}_{d} \mid A \in \Xi_{n, d}^{a p}\right\}$, which are compatible with the corresponding bases in $\mathbf{S}_{n, d}^{\mathfrak{c}}$ under the inclusion $\mathbf{U}_{n, d}^{\mathfrak{c}} \subset \mathbf{S}_{n, d}^{\mathfrak{c}}$.

1.3.2. An overview of Part 2, Generalizing the constructions in affine type $A$ and finite type $C$ [FL15] (see also [Lu00]), we introduce a comultiplication-like homomorphism $\Delta^{\mathfrak{c}}=$ $\Delta_{d^{\prime}, d^{\prime \prime}}^{\mathfrak{c}}: \mathbf{S}_{n, d}^{\mathfrak{c}} \rightarrow \mathbf{S}_{n, d^{\prime}}^{\mathfrak{c}} \otimes \mathbf{S}_{n, d^{\prime \prime}}$, for a composition $d=d^{\prime}+d^{\prime \prime}$. This further leads to a transfer map of affine type $C$ (which is an algebra homomorphism) $\phi_{d, d-n}^{\mathfrak{c}}: \mathbf{S}_{n, d}^{\mathfrak{c}} \rightarrow \mathbf{S}_{d-n, n}^{\mathfrak{c}}$, which is shown to preserve the Chevalley generators. Both homomorphisms $\Delta_{d^{\prime}, d^{\prime \prime}}^{\mathfrak{c}}$ and $\phi_{d, d-n}^{\mathfrak{c}}$ make sense on the level of Schur algebras instead of Lusztig algebras.

The algebra $\mathbf{U}_{n}^{\mathfrak{c}}$ is by definition a suitable subalgebra of the projective limit of the projective system $\left\{\left(\mathbf{U}_{n, d}^{\mathfrak{c}}, \phi_{d, d-n}^{\mathfrak{c}}\right)\right\}_{d \geqslant 1}$, just as $\mathbf{U}_{n}$ is a limit algebra for a similar affine type $A$ projective system. Recall by Proposition 2.3 .2 (due to Lusztig) we have an algebra isomorphism $\mathbf{U}_{n} \cong \mathbf{U}\left(\widehat{\mathfrak{s l}}_{n}\right)$. We show that the family of homomorphisms $\left\{\Delta_{d^{\prime}, d^{\prime \prime}}^{\mathfrak{c}}\right\}$ gives rise to a homomorphism $\Delta^{\mathfrak{c}}: \mathbf{U}_{n}^{\mathfrak{c}} \rightarrow \mathbf{U}_{n}^{\mathfrak{c}} \otimes \mathbf{U}_{n}$ and an injective homomorphism $\jmath_{n}: \mathbf{U}_{n}^{\mathfrak{c}} \rightarrow \mathbf{U}_{n}$, whose images on the Chevalley generators are explicitly given.

Theorem B (Theorem 6.1.4). The algebra $\mathbf{U}_{n}^{\mathfrak{c}}$ is a coideal subalgebra of $\mathbf{U}\left(\widehat{\mathfrak{s l}}_{n}\right)$, and the $\operatorname{pair}\left(\mathbf{U}\left(\hat{\mathfrak{s l}}_{n}\right), \mathbf{U}_{n}^{\mathfrak{c}}\right)$ forms a quantum symmetric pair of affine type in the sense of Letzter and Kolb [Ko14]. (The relevant involution is illustrated in Figure 1.)

Thanks to Theorem B, it makes sense to denote $\mathbf{U}_{n}^{\mathfrak{c}}=\mathbf{U}^{\mathfrak{c}}\left(\widehat{\mathfrak{s l}}_{n}\right)$; note the level for our affine type algebras is always zero. One can also formulate an idempotented form of $\mathbf{U}_{n}^{\mathfrak{c}}$, denoted by $\dot{\mathbf{U}}_{n}^{\mathfrak{c}}$ or $\dot{\mathbf{U}}^{\mathfrak{c}}\left(\widehat{\mathfrak{s l}}_{n}\right)$, which is analogous to the idempotented quantum groups as formulated in [BLM90, Lu93]. Following the approach of [Mc12] in the affine type $A$ setting and [LW15] in the finite type $B$ setting, we construct canonical basis for $\dot{\mathbf{U}}_{n}^{\mathfrak{c}}$ and establish its positivity with respect to the multiplication and a bilinear pairing of geometric origin. Following [FL15] in the finite type $B$ setting, we establish the positivity of the canonical basis for $\mathbf{U}_{n}^{\mathfrak{c}}$ with respect to the comultiplication.

Theorem C (Theorem 6.4.3, Theorem 6.4.5). The algebra $\dot{\mathbf{U}}_{n}^{\mathfrak{c}}$ admits a canonical basis $\dot{\mathbf{B}}_{n}^{\mathbf{c}}$. The structure constants of the canonical basis $\dot{\mathbf{B}}_{n}^{\mathfrak{c}}$ with respect to the multiplication and comultiplication are all positive, that is, they lie in $\mathbb{N}\left[v, v^{-1}\right]$ and so do they with respect to the bilinear pairing, that is, they lie in $\mathbb{N}\left[\left[v^{-1}\right]\right]$.

Recall in the finite type $C$ setting, there are geometric realizations of two quantum symmetric pairs (with superscripts $\jmath$ and $\imath$ ), the superscript $\jmath$ corresponds to the Dynkin diagram involution without fixed point and $\imath$ to the involution with a fixed point. The involution for $\widehat{\mathfrak{s l}}_{n}$ (where $n$ is even) in Figure 1 has no fixed point. In this paper we construct three more variants of quantum symmetric pairs arising from the affine type $C$ flags. The remaining three cases are labelled by superscripts $\jmath \imath, \imath, \imath$ and they correspond to involutions which are illustrated in Figures 2, 3 and 4, respectively (the superscript $\mathfrak{c}$ for the algebras above could be denoted by $\jmath$ ).

In each of the three new variants, we have counterparts of Theorems $\mathrm{A}$, $\mathrm{B}$ and C. The proofs are sometimes more difficult, as it is already clear in the finite rank $\imath$-version BLW14, LW15, FL15. 
There is also a totally different, purely algebraic, construction [BW16] of canonical bases for general quantum symmetric pairs of finite type (which is extendable to cover the QSP of affine type considered here). That approach does not establish the positivity of canonical bases.

1.3.3. An overview of Part 3. In contrast to the finite types, the Schur algebra $\mathbf{S}_{n, d}^{\mathfrak{c}}$ is not generated by the Chevalley generators in general, that is, $\mathbf{U}_{n, d}^{\mathfrak{c}}$ is a proper subalgebra of $\mathbf{S}_{n, d}^{\mathfrak{c}}$ (this phenomenon already happens in affine type $A$ [Lu99]). The next goal (Part 3) is to understand the limit algebra $\dot{\mathbf{K}}_{n}^{\mathfrak{c}}$ arising from the family of Schur algebras $\left\{\mathbf{S}_{n, d}^{\mathfrak{c}}\right\}_{d \geqslant 1}$ as well as its $\imath \imath \imath \jmath, \imath$-variants. One key difficulty we encounter here is that the Schur algebras $\mathbf{S}_{n, d}^{\mathfrak{c}}$ do not have any obvious (finite) generating set to start with, and this makes it tricky to understand the stabilization.

To that end, we introduce a new idea by imbedding $\mathbf{S}_{n, d}^{\mathfrak{c}}$ into the Lusztig algebra $\mathbf{U}_{\breve{n}, d}^{\mathfrak{c}}$ (with $\breve{n}=n+2$ ). The imbedding $\mathbf{S}_{n, d}^{\mathfrak{c}} \rightarrow \mathbf{U}_{\breve{n}, d}^{\mathfrak{c}}$ is constructed as an imbedding $\mathbf{S}_{n, d}^{\mathfrak{c}} \rightarrow \mathbf{S}_{\breve{n}, d}^{\mathfrak{c}}$ (in a way similar to the embedding $\mathbf{S}_{\mathfrak{n}, d}^{\jmath} \rightarrow \mathbf{S}_{n, d}^{\mathfrak{c}}$ earlier) which factors through $\mathbf{U}_{\breve{n}, d}^{\mathfrak{c}}$. As Lusztig algebras have a nice set of Chevalley generators and they are well understood in Part 1 and Part 2, we gain insights about $\mathbf{S}_{n, d}^{\mathfrak{c}}$ this way.

One first result which we obtain via such an imbedding is to establish a (bar invariant) monomial basis $\left\{\mathbf{f}_{A} \mid A \in \Xi_{n, d}\right\}$ for $\mathbf{S}_{n, d}^{\mathfrak{c}}$, and we see that $\mathbf{S}_{n, d}^{\mathfrak{c}}$ is generated by the standard basis elements $[A]_{d}$ with $A$ tridiagonal. (In affine type $A$, it was first shown [DF13] that the Schur algebra is generated by the standard basis elements ${ }^{\mathfrak{a}}[A]_{d}$ for $A$ bidiagonal.) In our affine type $C$ setting, thanks to the centrosymmetry condition of the matrices $A$ parametrizing the basis of $\mathbf{S}_{n, d}^{\mathfrak{c}}$, the appearance of tridiagonal matrices parametrizing a generating set is perhaps not surprising. It does make any possible multiplication formula in affine type $C$ with $[A]$ for $A$ tridiagonal enormously complicated.

The imbedding $\mathbf{S}_{n, d}^{\mathfrak{c}} \rightarrow \mathbf{U}_{\breve{n}, d}^{\mathfrak{c}}$ and the monomial basis for $\mathbf{S}_{n, d}^{\mathfrak{c}}$ further allow us to study fruitfully the stabilization as $d$ goes to infinity of the multiplication, comultiplication, and bar involution on $\mathbf{S}_{n, d}^{\mathfrak{c}}$. The stabilization properties for $\mathbf{S}_{n, d}^{\mathfrak{c}}$ allow us to introduce a limit algebra $\dot{\mathbf{K}}_{n}^{\mathfrak{c}}$ and establish its main properties.

Theorem D (Theorems 9.4.6, 9.7.3). The algebra $\dot{\mathbf{K}}_{n}^{\mathfrak{c}}$ admits a standard basis $\left\{[A] \mid A \in \widetilde{\Xi}_{n}\right\}$, a monomial basis $\left\{\mathbf{f}_{A} \mid A \in \widetilde{\Xi}_{n}\right\}$, and a stably canonical basis $\left\{\{A\} \mid A \in \widetilde{\Xi}_{n}\right\}$. Moreover, there is a natural surjective algebra homomorphism $\Psi_{n, d}: \dot{\mathbf{K}}_{n}^{\mathfrak{c}} \rightarrow \mathbf{S}_{n, d}^{\mathfrak{c}}$ which sends each stably canonical basis element to a canonical basis element or zero.

In a completely analogous way and as a byproduct, we can formulate the stabilization properties of the family of Schur algebras $\mathbf{S}_{n, d}$ of affine type $A$ and introduce its stabilization algebra $\dot{\mathbf{K}}_{n}$, and prove a theorem for $\dot{\mathbf{K}}_{n}$ analogous to Theorem D. Such results in affine type $A$ were first obtained in [DF13, DF14] by a completely different and algebraic approach, and they also identify $\dot{\mathbf{K}}_{n}$ as the idempotented quantum affine $\hat{\mathfrak{g l}}_{n}$. Our geometric approach here offers a shortcut to some main results in loc. cit. and obtains new results on the comultiplication structure.

The stabilization property of the comultiplication on $\mathbf{S}_{n, d}^{\mathfrak{c}}$ leads to the following.

Theorem E (Propositions 9.6.1, 9.6.2, Remark 9.6.3). The pair $\left(\dot{\mathbf{K}}_{n}, \dot{\mathbf{K}}_{n}^{\mathfrak{c}}\right)$ forms a quantum symmetric pair (in an idempotented form). 
Similarly, the other families of Schur algebras $\left\{\mathbf{S}_{\mathfrak{n}, d}^{\jmath \imath}\right\}_{d},\left\{\mathbf{S}_{\mathfrak{n}, d}^{\imath \jmath}\right\}_{d}$, and $\left\{\mathbf{S}_{\eta, d}^{\imath \imath}\right\}_{d}$ admit similar stabilizations which lead to limit algebras $\dot{\mathbf{K}}_{\mathfrak{n}}^{\jmath \imath}, \dot{\mathbf{K}}_{\mathfrak{n}}^{\imath \jmath}, \dot{\mathbf{K}}_{\eta}^{\imath \imath}$, respectively. We also establish the counterparts of Theorems D and E for the algebras $\dot{\mathbf{K}}_{\mathfrak{n}}^{\jmath \imath}, \dot{\mathbf{K}}_{\mathfrak{n}}^{\imath \jmath}, \dot{\mathbf{K}}_{\eta}^{\imath \imath}$. In the process, we actually establish the following interrelations in Section 10.5 (where one finds the precise definition of subquotients) among the algebras $\dot{\mathbf{K}}_{n}^{\mathfrak{c}}, \dot{\mathbf{K}}_{\mathfrak{n}}^{\jmath}, \dot{\mathbf{K}}_{\mathfrak{n}}^{\imath \jmath}, \dot{\mathbf{K}}_{\eta}^{\imath \imath}$ in a conceptual way.

Theorem F (Proposition 9.8.2, Theorems 10.3.6, 10.4.1, 10.5.1). We have the following diagram of subquotient constructions (sq stands for subquotients):

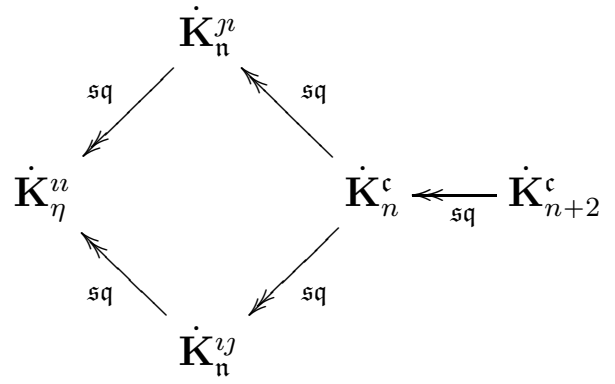

Moreover, all the subquotient constructions are compatible with the stably canonical bases.

We have developed a Hecke-algebraic approach in a companion paper [FLLLW] simultaneously, which redevelops some of the main results of Part 3 of this paper in a completely different way. See also [FL17] for a third approach based on an explicit multiplication formula on tri-diagonal standard basis elements.

\subsection{THE ORGANIZATION}

The paper is divided into three parts. Part 1 consists of Chapters 2,5, and it deals with the Schur algebras and Lusztig algebras arising from convolution algebras on pairs of partial flags of affine type $C$. Part 2 consists of Chapters 6-8, and it studies the limit algebras of each of the four families of Lusztig algebras and identifies them as (idempotented) coideal subalgebras of the quantum affine $\mathfrak{s l}$. Part 3 consists of Chapters 9, $[0$, and it treats the stabilization algebras arising from the four families of Schur algebras, and identify them as (idempotented) coideal subalgebras of the quantum affine $\mathfrak{g l}_{n}$.

In the somewhat preliminary Chapter 2, which is exclusively on affine type $A$, we review the constructions of [Lu99] in affine type $A$ and set up the type $A$ notations. We formulate Lusztig algebra $\mathbf{U}_{n, d}$ as the (proper) subalgebra of the Schur algebra $\mathbf{S}_{n, d}$ generated by Chevalley generators. A new result in this Chapter is a geometric construction of a monomial basis for $\mathbf{U}_{n, d}$ and then for $\mathbf{U}\left(\widehat{\mathfrak{s l}}{ }_{n}\right)$. This makes our approach here and further generalization in affine type $C$ below quite different from those in [Mc12, SV00]. In particular, the approach here does not rely on the crystal basis theory of Kashiwara and Ringel-Hall algebras.

Before proceeding to the remaining chapters, we recommend the reader to browse Appen$\operatorname{dix}$ A. In Appendix A, we review and expand the geometric constructions from [BKLW14, FL15] in finite type $C$. Recall most of the results in loc. cit. were formulated in detail in the geometric setting of finite type $B$.

From now on we take $n$ to be a positive even integer.

In Chapter 3, we present lattice models for the variety $\mathcal{Y}^{\mathfrak{c}}$ of complete flags of affine type $C$, following [Sa99], [H99] and [Lu03]. We also formulate a variety $\mathcal{X}_{n, d}^{\mathfrak{c}}$ of $n$-step flags of 
affine type $C$. Then we classify the orbits of products $\mathcal{X}_{n, d}^{\mathfrak{c}} \times \mathcal{Y}^{\mathfrak{c}}$ and $\mathcal{X}_{n, d}^{\mathfrak{c}} \times \mathcal{X}_{n, d}^{\mathfrak{c}}$ under the diagonal action of the loop symplectic group.

In Chapter 4, we study the Schur algebra $\mathbf{S}_{n, d}^{\mathfrak{c}}$ arising from the convolution algebra of pairs of $n$-step flags of affine type $C$. We present multiplication formulas in $\mathbf{S}_{n, d}^{\mathfrak{c}}$ with the Chevalley generators and with their divided powers. We then specify some general scenarios where these multiplication formulas produce a leading term with coefficient 1 . The results in this chapter are local in the sense that they are analogous to the results in finite types $A$ and $C$.

In Chapter 5, we introduce the Lusztig algebra $\mathbf{U}_{n, d}^{\mathfrak{c}}$ as the (proper) subalgebra of the Schur algebra $\mathbf{S}_{n, d}^{\mathfrak{c}}$ generated by Chevalley generators. We then introduce a coideal algebra type structure which involves both Schur algebras (and respectively, Lusztig algebras) of affine types $C$ and $A$. This leads to an imbedding $\jmath_{n, d}$ from $\mathbf{S}_{n, d}^{\mathfrak{c}}$ to $\mathbf{S}_{n, d}$, and also from $\mathbf{U}_{n, d}^{\mathfrak{c}}$ to $\mathbf{U}_{n, d}$. The canonical basis and monomial basis are shown to be compatible with the inclusion $\mathbf{U}_{n, d}^{\mathfrak{c}} \subset \mathbf{S}_{n, d}^{\mathfrak{c}}$.

In Chapter 6, we introduce the transfer maps $\phi_{d, d-n}^{\mathfrak{c}}$ on Schur algebras $\mathbf{S}_{n, d}^{\mathfrak{c}}$ and Lusztig algebras $\mathbf{U}_{n, d}^{\mathfrak{c}}$. We then construct algebras $\mathbf{U}_{n}^{\mathfrak{c}}\left(\right.$ or $\dot{\mathbf{U}}_{n}^{\mathfrak{c}}$ ) from the projective system of algebras $\left\{\left(\mathbf{U}_{n, d}^{\mathfrak{c}}, \phi_{d, d-n}^{\mathfrak{c}}\right)\right\}_{d \geqslant 0}$. We show that $\mathbf{U}_{n}^{\mathfrak{c}}\left(\right.$ or $\left.\dot{\mathbf{U}}_{n}^{\mathfrak{c}}\right)$ is isomorphic to an (idempotented) coideal subalgebra of $\mathbf{U}\left(\widehat{\mathfrak{s l}}_{n}\right)$, and $\left(\mathbf{U}\left(\widehat{\mathfrak{s l}}_{n}\right), \mathbf{U}_{n}^{\mathfrak{c}}\right)$ forms an affine quantum symmetric pair. The canonical basis of $\dot{\mathbf{U}}_{n}^{\mathfrak{c}}$ is established and shown to admit positivity with respect to multiplication, comultiplication, and a bilinear pairing.

In the remainder of the Introduction we set $\mathfrak{n}=n-1$ (which is odd) and $\eta=n-2$ (which is even).

In Chapter 7 and Chapter 8 , we present several more projective systems $\left\{\left(\mathbf{U}_{\mathfrak{n}, d}^{\jmath n}, \phi_{d, d-\mathfrak{n}}^{\mathfrak{\jmath}}\right)\right\}_{d \geqslant 0}$, $\left\{\left(\mathbf{U}_{\mathfrak{n}, d}^{\imath \jmath}, \phi_{d, d-\mathfrak{n}}^{\imath \jmath}\right)\right\}_{d \geqslant 0}$, and $\left\{\left(\mathbf{U}_{\eta, d}^{\imath \imath}, \phi_{d, d-\eta}^{\imath \imath}\right)\right\}_{d \geqslant 0}$. We emphasize that each of these Lusztig algebras arises from convolution algebras of geometric origin. We obtain the limit algebras $\mathbf{U}_{\mathfrak{n}}^{\jmath \imath}, \mathbf{U}_{\mathfrak{n}}^{\imath \jmath}$, $\mathbf{U}_{\eta}^{\imath \imath}$ and their idempotented counterparts. We show that $\mathbf{U}_{\mathfrak{n}}^{\jmath}$ (respectively, $\mathbf{U}_{\mathfrak{n}}^{\imath}$, or $\mathbf{U}_{\eta}^{\imath \imath}$ ) is isomorphic to a coideal subalgebra of $\mathbf{U}\left(\widehat{\mathfrak{s l}}_{\mathfrak{n}}\right)$ (respectively, $\mathbf{U}\left(\widehat{\mathfrak{s l}}_{\mathfrak{n}}\right)$ or $\mathbf{U}\left(\widehat{\mathfrak{s l}}_{\eta}\right)$ ). The monomial and canonical bases of $\mathbf{U}_{\mathfrak{n}, d}^{\jmath \imath}, \mathbf{U}_{\mathfrak{n}, d}^{\imath \jmath}$, and $\mathbf{U}_{\eta, d}^{\imath \imath}$ are established by relating to their counterparts for $\mathbf{U}_{n, d}^{\mathfrak{c}}$. The canonical bases of $\dot{\mathbf{U}}_{\mathfrak{n}}^{\jmath \imath}, \dot{\mathbf{U}}_{\mathfrak{n}}^{\imath \jmath}$ and $\dot{\mathbf{U}}_{\eta}^{\imath \imath}$ are established and shown to admit positivity with respect to multiplication, comultiplication, and a bilinear pairing.

In Chapter 9, we study the stabilization properties of the family of Schur algebras $\mathbf{S}_{n, d}^{\mathfrak{c}}$ (as $d$ varies). To overcome the difficulty of working with the Schur algebra $\mathbf{S}_{n, d}^{\mathfrak{c}}$ which does not have a good finite generating set, we study $\mathbf{S}_{n, d}^{\mathfrak{c}}$ via an imbedding into a Lusztig algebra of higher rank. This allows us to understand generating sets, monomial bases, multiplication, comultiplication and bar operators of the Schur algebras and their stabilization properties in a conceptual way and lift all these structures to a stabilization algebra $\dot{\mathbf{K}}_{n}^{\mathfrak{c}}$. We show that $\dot{\mathbf{K}}_{n}^{\mathfrak{c}}$ admits a stably canonical basis, and the pair $\left(\dot{\mathbf{K}}_{n}, \dot{\mathbf{K}}_{n}^{\mathfrak{c}}\right)$ forms a quantum symmetric pair in an idempotented form, where $\dot{\mathbf{K}}_{n}$ is isomorphic to the idempotented quantum affine $\mathfrak{g l}_{n}$.

In Chapter 10, we formulate the main results for the stabilizations of the remaining 3 families of Schur algebras of types $\jmath, \imath \jmath, \imath$, following the blueprints in Chapter 9. Moreover, we establish interrelations among all the stabilization algebras $\dot{\mathbf{K}}_{n}^{\mathfrak{c}}, \dot{\mathbf{K}}_{\mathfrak{n}}^{\imath \jmath}, \dot{\mathbf{K}}_{\mathfrak{n}}^{\imath \jmath}$, and $\dot{\mathbf{K}}_{\eta}^{\imath \imath}$ of types $\jmath \jmath, \jmath \imath, \imath \jmath, \imath$, and among their stably canonical bases.

Notation: $\mathbb{N}=\{0,1,2, \ldots\}$. 
Acknowledgement. We thank Huanchen Bao, Jie Du and Xuhua He for helpful discussions. We thank the following institutions whose support and hospitality help to facilitate the progress and completion of this project: East China Normal University (Shanghai), Institute of Mathematics, Academia Sinica (Taipei), University of Virginia. ZF is partially supported by the Fundamental Research Funds for the central universities (grant No. GK2110260131), and by the NSF of China (grant No. 11671108). LL is partially supported by the Science and Technology Commission of Shanghai Municipality (grant No. 13dz2260400) and the NSF of China (grant No. 11671139). WW is partially supported by the NSF grant DMS-1405131 and DMS-1702254. 
Part 1. Affine flag varieties, Schur algebras, and Lusztig algebras 


\section{CHAPTER 2}

\section{Constructions in affine type $A$}

This chapter is preliminary in nature. Most of it has been well known [Lu99, Lu00, SV00, Mc12] (also cf. [DF14]). However we present a new geometric construction of a monomial basis (and hence canonical basis) for the modified quantum group $\dot{\mathbf{U}}\left(\widehat{\mathfrak{s l}}_{n}\right)$, in analogy to the one in [BLM90, Proposition 3.9], without use of crystal basis [K91 and the theory of Ringel-Hall algebras [R90], (see also [Sch06, DDPW08, DDF12], [VV99, LL15]).

\subsection{LATtice PRESENTATiOn of AFFine FlaG VARIETIES OF TYPE $A$}

Let $k$ be a finite field of $q$ elements, where $q$ is a prime power. Let $F=k((\varepsilon))$ be the field of formal Laurent series over $k$ and $\mathfrak{o}=k[[\varepsilon]]$ the ring of formal power series. Let $d$ be a positive integer. Let $\mathrm{GL}_{F}(d)$ (respectively, $\mathrm{GL}_{\mathfrak{o}}(d), \mathrm{GL}_{k}(d)$ ) be the invertible $d \times d$ matrices with coefficients in $F$ (respectively, $\mathfrak{o}, k$ ). Consider a reduction mod- $\varepsilon$ map $\left.\mathrm{ev}\right|_{\varepsilon=0}: \mathrm{GL}_{\mathfrak{o}}(d) \rightarrow \mathrm{GL}_{k}(d), \quad \varepsilon \mapsto 0$. The parahoric subgroups of $\mathrm{GL}_{F}(d)$ are inverse images of parabolic subgroups of $\mathrm{GL}_{k}(d)$ under ev $\left.\right|_{\varepsilon=0}$, and the parahoric subgroups which are inverse images of Borel subgroups are called Iwahori subgroups. The affine partial flag of type $A$ is then defined to be the homogeneous space $\mathrm{GL}_{F}(d) / P$ where $P$ is a parahoric subgroup.

Let $V$ be an $F$-vector space of dimension $d$. A free $\mathfrak{o}$-submodule $\mathcal{L}$ of $V$ of rank $d$ is called a lattice in $V$. Let $\mathcal{Y}^{\mathfrak{a}}$ be the set of all lattice chains $L=\left(L_{i}\right)_{i \in \mathbb{Z}}$ where each $L_{i}$ is a lattice in $V$, such that $L_{i-1} \subset L_{i}$ and $L_{i-d}=\varepsilon L_{i}$ for all $i \in \mathbb{Z}$. We fix a basis $\left\{e_{1}, \ldots, e_{d}\right\}$ for $V$, and we set

$$
e_{m}=\varepsilon^{-s} e_{i}, \quad \text { if } m=s d+i \text { for } i \in[1, d] .
$$

Then we have a total order for $\left(e_{m}\right)_{m \in \mathbb{Z}}$ as follows:

$$
\ldots, \varepsilon e_{1}, \ldots, \varepsilon e_{d}, e_{1}, \ldots, e_{d}, \varepsilon^{-1} e_{1}, \ldots, \varepsilon^{-1} e_{d}, \ldots
$$

Clearly,

$$
\mathrm{E}_{0}=\mathfrak{o} e_{1} \oplus \cdots \oplus \mathfrak{o} e_{d}
$$

is a lattice in $V$. More generally, for $m=s d+i$ with $1 \leqslant i \leqslant d$, we define the lattice

$$
\begin{aligned}
\mathrm{Ł}_{m} & =\left[e_{m+1}, \ldots, e_{m+d}\right]_{\mathfrak{o}} \\
& =\mathfrak{o} \varepsilon^{-s} e_{i+1} \oplus \cdots \oplus \mathfrak{o} \varepsilon^{-s} e_{d} \oplus \mathfrak{o} \varepsilon^{-s-1} e_{1} \oplus \cdots \oplus \mathfrak{o} \varepsilon^{-s-1} e_{i} .
\end{aligned}
$$

We set $\mathrm{E}=\left(\mathrm{E}_{m} \mid m \in \mathbb{Z}\right)$ to be the standard lattice chain. There exists a surjective map

$$
\mathrm{GL}_{F}(d) \rightarrow \mathcal{Y}^{\mathfrak{a}}, \quad g \mapsto g . .
$$

It is clear that the stabilizer $\mathbf{I}^{\mathfrak{a}}$ of $€$ in $\mathrm{GL}_{F}(d)$ consists exactly of the mod- $\varepsilon$ upper triangular matrices. Thus $\mathbf{I}^{\mathfrak{a}}$ is an Iwahori subgroup of $\mathrm{GL}_{F}(d)$. We thus have the identification of affine flag variety of type $A$ :

$$
\mathrm{GL}_{F}(d) / \mathbf{I}_{16}^{\mathfrak{a}} \longrightarrow \mathcal{Y}^{\mathfrak{a}}
$$


There are similar lattice chain models for the partial flag varieties of type $A$.

\subsection{MONOMIAL BASIS FOR QUANTUM AFFine $\mathfrak{s l}_{n}$}

In this section, we shall construct an explicit monomial basis for quantum affine $\mathfrak{s l}_{n}$ (the construction here will be generalized in latter chapters).

For the partial flag cases, the treatment is similar. More generally, we consider the set $\mathcal{X}_{n, d}$ of $n$-periodic lattice chains in $V$. Here $\operatorname{dim}_{F} V=d$ and a sequence $L=\left(L_{i}\right)_{i \in \mathbb{Z}}$ of lattices in $V$ is called an $n$-periodic lattice chain if $L_{i} \subseteq L_{i+1}$ and $L_{i}=\varepsilon L_{i+n}$ for all $i \in \mathbb{Z}$. The group $\mathrm{GL}_{F}(d)$ acts naturally on $\mathcal{X}_{n, d}$ from the left, and then acts on the product $\mathcal{X}_{n, d} \times \mathcal{X}_{n^{\prime}, d}$ diagonally, for a pair $\left(n, n^{\prime}\right)$ of positive integers.

Let $\Theta_{n \mid n^{\prime}, d}$ be the set of all matrices $A=\left(a_{i j}\right)_{i, j \in \mathbb{Z}}$ with non-negative integer entries satisfying the following conditions:

$$
\text { (i) } a_{i j}=a_{i+n, j+n^{\prime}}(\forall i, j \in \mathbb{Z}) ; \quad(i i) \sum_{i=i_{0}}^{i_{0}+n-1} \sum_{j \in \mathbb{Z}} a_{i j}=d, \text { for each (or for all) } i_{0} \in \mathbb{Z} .
$$

The condition $(i i)$ can be equivalently replaced by $\left(i i^{\prime}\right)$ below:

$\left(i i^{\prime}\right)$ For any $j_{0} \in \mathbb{Z}, \sum_{j=j_{0}}^{j_{0}+n^{\prime}-1} \sum_{i \in \mathbb{Z}} a_{i j}=d$.

A matrix $A$ in $\Theta_{n \mid n^{\prime}, d}$ automatically satisfies that, for any $i \in \mathbb{Z}$, the sets $\left\{j \in \mathbb{Z} \mid a_{i j} \neq 0\right\}$ and $\left\{j \in \mathbb{Z} \mid a_{j i} \neq 0\right\}$ are finite.

Following [Lu99], the $\mathrm{GL}_{F}(d)$-orbits in $\mathcal{X}_{n, d} \times \mathcal{X}_{n^{\prime}, d}$ are parametrized by the set $\Theta_{n \mid n^{\prime}, d}$. More precisely, to a pair of $n$-periodic lattices $\left(L, L^{\prime}\right)$, we define a matrix $A=\left(a_{i j}\right)_{i, j \in \mathbb{Z}}$ where

$$
a_{i j}=\operatorname{dim}_{k} L_{i} \cap L_{j}^{\prime} /\left(L_{i-1} \cap L_{j}^{\prime}+L_{i} \cap L_{j-1}^{\prime}\right), \quad(\forall i, j \in \mathbb{Z}) .
$$

This defines a bijection $\mathrm{GL}_{F}(d) \backslash \mathcal{X}_{n, d} \times \mathcal{X}_{n^{\prime}, d} \leftrightarrow \Theta_{n \mid n^{\prime}, d}$. Let $\mathcal{O}_{A}$ denote the associated $\mathrm{GL}_{F}(d)$ orbit indexed by $A$. We are mostly interested in the case when $n^{\prime}=n$, and we shall write

$$
\Theta_{n, d}=\Theta_{n \mid n, d} .
$$

We set

$$
\Lambda_{n, d}=\left\{\lambda=\left(\lambda_{i}\right)_{i \in \mathbb{Z}} \in \mathbb{N}^{\mathbb{Z}} \mid \lambda_{i}=\lambda_{i+n}, \forall i \in \mathbb{Z} ; \sum_{1 \leqslant i \leqslant n} \lambda_{i}=d\right\} .
$$

To each matrix $A \in \Theta_{n, d}$, we define its row/column sum vectors $\operatorname{ro}(A)=\left(\operatorname{ro}(A)_{i}\right)_{i \in \mathbb{Z}}$ and $\operatorname{co}(A)=\left(\operatorname{co}(A)_{i}\right)_{i \in \mathbb{Z}}$ in $\Lambda_{n, d}$ by

$$
\operatorname{ro}(A)_{i}=\sum_{j \in \mathbb{Z}} a_{i j}, \quad \operatorname{co}(A)_{j}=\sum_{i \in \mathbb{Z}} a_{i j}(\forall i, j \in \mathbb{Z})
$$

Let $A, B, C \in \Theta_{n, d}$, we fix $L, L^{\prime} \in \mathcal{X}_{n, d}$ such that $\operatorname{dim}_{k} L_{i} / L_{i-1}=\operatorname{ro}(A)_{i}$ and $\operatorname{dim}_{k} L_{j}^{\prime} / L_{j-1}^{\prime}=$ $\operatorname{co}(B)_{j}$ for all $i, j \in \mathbb{Z}$. We set

$$
g_{A, B}^{C}(\sqrt{q})=\#\left\{\tilde{L} \in \mathcal{X}_{n, d} \mid(L, \tilde{L}) \in \mathcal{O}_{A},\left(\tilde{L}, L^{\prime}\right) \in \mathcal{O}_{B},\left(L, L^{\prime}\right) \in \mathcal{O}_{C}\right\} .
$$

By [Lu99], $g_{A, B}^{C}(\sqrt{q})$ is independent of the choices of $L, L^{\prime}$ and is the specialization of a polynomial $g_{A, B}^{C}(v) \in \mathbb{Z}\left[v, v^{-1}\right]$ at $v=\sqrt{q}$. Note that $g_{A, B}^{C}=0$ for all but finitely many $C$.

We set $\mathcal{A}=\mathbb{Z}\left[v, v^{-1}\right]$. The affine Schur $\mathcal{A}$-algebra of type $A$, denoted by $\mathbf{S}_{n, d ; \mathcal{A}}$, is by definition the (generic) convolution algebra $\mathcal{A}_{\mathrm{GL}_{F}(d)}\left(\mathcal{X}_{n, d} \times \mathcal{X}_{n, d}\right)$. Denote by $e_{A}$ the 
characteristic function of the orbit $\mathcal{O}_{A}$, for $A \in \Theta_{n, d}$. Then the algebra $\mathbf{S}_{n, d ; \mathcal{A}}$ is a free $\mathcal{A}$ module with an $\mathcal{A}$-basis $\left\{e_{A} \mid A \in \Theta_{n, d}\right\}$, with multiplication given by $e_{A} * e_{B}=\sum_{C} g_{A, B}^{C}(v) e_{C}$. We then set

$$
\mathbf{S}_{n, d}=\mathbb{Q}(v) \otimes_{\mathcal{A}} \mathbf{S}_{n, d ; \mathcal{A}}
$$

To $A \in \Theta_{n, d}$, we define

$$
d_{A}^{\mathfrak{a}}=\sum_{1 \leqslant i \leqslant n, i \geqslant k, j<l} a_{i j} a_{k l}
$$

and

$$
[A]=v^{-d_{A}^{\mathfrak{a}}} e_{A} .
$$

The set $\left\{[A] \mid A \in \Theta_{n, d}\right\}$ is the standard basis of $\mathbf{S}_{n, d}$. Let $\left\{\mathfrak{a}\{A\}_{d} \mid A \in \Theta_{n, d}\right\}$ be the canonical basis of $\mathbf{S}_{n, d}\left[\mathrm{Lu} 99\right.$. Given $i, j \in \mathbb{Z}$, let $E^{i j}$ be the $\mathbb{Z} \times \mathbb{Z}$ matrix whose $(k, \ell)$ th entries are 1 , for all $(k, \ell) \equiv(i, j)(\bmod n)$, and 0 otherwise; that is,

$$
E^{i j}=\left(\mathcal{E}_{k, \ell}\right)_{k, \ell \in \mathbb{Z}}, \quad \text { where } \mathcal{E}_{k, \ell}=1 \text { if }(k, \ell) \equiv(i, j)(\bmod n) \text {, otherwise } \mathcal{E}_{k, \ell}=0 .
$$

Definition 2.2.1. The subalgebra of $\mathbf{S}_{n, d}$ generated by the standard basis elements $[X]$ such that either $X$ or $X-E^{i, i+1}$ or $X-E^{i+1, i}$ is diagonal, is denoted by $\mathbf{U}_{n, d}$ and called Lusztig algebra (of affine type $A$ ).

Let $\mathbf{U}_{n, d ; \mathcal{A}}$ be the subalgebra of $\mathbf{S}_{n, d ; \mathcal{A}}$ generated by the standard basis element $[X]$ such that either $X-R E^{i, i+1}$ or $X-R E^{i+1, i}$ is diagonal, for various $R \in \mathbb{N}$. For each $\lambda \in \Lambda_{n, d}$, let $D_{\lambda}$ be the diagonal matrix in $\Theta_{n, d}$ whose diagonal is $\lambda$. For each $R \in \mathbb{N}, i \in \mathbb{Z}$, we set

$$
\mathbf{E}_{i}^{(R)}=\sum[X], \quad \mathbf{F}_{i}^{(R)}=\sum[X], \quad \mathbf{H}_{i}^{ \pm 1}=\sum_{\lambda \in \Lambda_{n, d}} v^{ \pm \lambda_{i}}\left[D_{\lambda}\right], \quad \mathbf{K}_{i}^{ \pm 1}=\mathbf{H}_{i+1}^{ \pm 1} \mathbf{H}_{i}^{\mp 1}
$$

where the first and second sums run over all $X$ such that $X-R E^{i+1, i}$ and $X-R E^{i, i+1}$ are diagonal, respectively. Clearly, we have $\mathbf{E}_{i}^{(R)}=\mathbf{E}_{j}^{(R)}, \mathbf{F}_{i}^{(R)}=\mathbf{F}_{j}^{(R)}, \mathbf{H}_{i}^{ \pm 1}=\mathbf{H}_{j}^{ \pm 1}$ and $\mathbf{K}_{i}^{ \pm 1}=\mathbf{K}_{j}^{ \pm 1}$ for all $i \equiv j(\bmod n)$. For convenience, we also set $1_{\lambda}=\left[D_{\lambda}\right]$,

It is known from [Lu99] that $\mathbf{U}_{n, d ; \mathcal{A}}$ is an $\mathcal{A}$-lattice of $\mathbf{U}_{n, d}$ and generated by $\mathbf{E}_{i}^{(R)}, \mathbf{F}_{i}^{(R)}$ and $\mathbf{K}_{i}^{ \pm 1}$ for all $i$ and $R \in \mathbb{N}$. Recall a $\mathbb{Z} \times \mathbb{Z}$-matrix $A=\left(a_{i j}\right)$ is aperiodic if

$$
\text { for any } p \in \mathbb{Z}-\{0\} \text { there exists } k \in \mathbb{Z} \text { such that } a_{k, k+p}=0 \text {. }
$$

We denote by $\Theta_{n, d}^{a p}$ the set of all aperiodic matrices in $\Theta_{n, d}$. Lusztig [Lu99] showed that $\mathbf{U}_{n, d}$ is a proper subalgebra of $\mathbf{S}_{n, d}$ and further the subset $\left\{\mathfrak{a}\{A\}_{d} \mid A \in \Theta_{n, d}^{a p}\right\}$ of the canonical basis of $\mathbf{S}_{n, d}$ form a canonical basis ${ }^{\mathfrak{a}}\{A\}_{d}$ of $\mathbf{U}_{n, d}$. Note that the latter result is completely nontrivial since the standard basis element $[A]$ for $A$ aperiodic is not in $\mathbf{U}_{n, d}$ in general.

For $a \in \mathbb{Z}$ and $b \in \mathbb{N}$, we define

$$
\left[\begin{array}{l}
a \\
b
\end{array}\right]=\prod_{1 \leqslant i \leqslant b} \frac{v^{2(a-i+1)}-1}{v^{2 i}-1}, \quad \text { and } \quad[a]=\left[\begin{array}{l}
a \\
1
\end{array}\right] .
$$


We define two partial orders " $\leqslant$ alg" and " $\leqslant$ " on $\Theta_{n, d}$ as follows. For any $A=\left(a_{i j}\right), A^{\prime}=$ $\left(a_{i j}^{\prime}\right) \in \Theta_{n, d}$, let

$$
\begin{gathered}
A \leqslant \text { alg } A^{\prime} \Longleftrightarrow \sum_{k \leqslant i, l \geqslant j} a_{k l} \leqslant \sum_{k \leqslant i, l \geqslant j} a_{k l}^{\prime}, \quad \forall i<j, \\
\sum_{k \geqslant i, l \leqslant j} a_{k l} \leqslant \sum_{k \geqslant i, l \leqslant j} a_{k l}^{\prime}, \quad \forall i>j . \\
A \leqslant A^{\prime} \Longleftrightarrow A \leqslant \text { alg } A^{\prime}, \operatorname{ro}(A)=\operatorname{ro}\left(A^{\prime}\right), \operatorname{co}(A)=\operatorname{co}\left(A^{\prime}\right) .
\end{gathered}
$$

We further say that " $A<$ alg $A^{\prime \prime}$ ", (respectively, " $A<A^{\prime \prime}$ ") if $A \leqslant$ alg $A^{\prime}$ (respectively, $A \leqslant A^{\prime}$ ) and $A \neq A^{\prime}$. For convenience, we write " $[A]+$ lower terms" to stand for " $[A]$ plus a linear sum of various $[B]$ with $B<A$ ".

The following lemma is a slightly stronger affine version of [BLM90, Lemma 3.8], which is used to obtain an affine analogue of [BLM90, Proposition 3.9] for quantum affine $\mathfrak{s l}_{n}$.

Lemma 2.2.2. Let $A, B, C \in \Theta_{n, d}$ and $R$ be a positive integer.

(1) Assume that $B-R E^{h, h+1}$ is diagonal for some $h \in[1, n]$ and $\operatorname{co}(B)=\operatorname{ro}(A)$. Assume further that $R=R_{0}+\cdots+R_{l}$ and the matrix $A$ satisfies the following conditions:

$a_{h j}=0, \forall j \geqslant k ; a_{h+1, k+i}=R_{i}, i \in[1, l], a_{h+1, k} \geqslant R_{0}, a_{h+1, j}=0, \forall j>k+l$.

Then we have

$$
[B] *[A]=\left[A+\sum_{i=0}^{l} R_{i}\left(E^{h, k+i}-E^{h+1, k+i}\right)\right]+\text { lower terms. }
$$

(2) Assume that $C-R E^{h+1, h}$ is diagonal for some $h \in[1, n]$ and $\operatorname{co}(C)=\operatorname{ro}(A)$. Assume further that $R=R_{0}+\cdots+R_{l}$ and $A$ satisfies the following conditions:

$a_{h j}=0, \forall j<k, a_{h, k+i}=R_{i}, i \in[0, l-1], a_{h, k+l} \geqslant R_{l} ; a_{h+1, j}=0, \forall j \leqslant k+l$.

Then we have

$$
[C] *[A]=\left[A-\sum_{i=0}^{l} R_{i}\left(E^{h, k+i}-E^{h+1, k+i}\right)\right]+\text { lower terms. }
$$

Proof. By [Lu99, Section 3], we have

$$
[B] *[A]=\sum_{t} v^{\beta(t)} \prod_{u \in \mathbb{Z}} \overline{\left[\begin{array}{c}
a_{h u}+t_{u} \\
t_{u}
\end{array}\right]}\left[A+\sum_{u \in \mathbb{Z}} t_{u}\left(E^{h u}-E^{h+1, u}\right)\right],
$$

where $\beta(t)=\sum_{j \geqslant u} a_{h j} t_{u}-\sum_{j>u} a_{h+1, j} t_{u}+\sum_{j<u} t_{j} t_{u}$. Here the bar is the involution on $\mathbb{Q}(v)$ defined by $\bar{v}=v^{-1}$. Observe that $A+\sum_{i=0}^{l} R_{i}\left(E^{h, k}-E^{h+1, k}\right)$ is the leading term for the right hand side of (2.2.10).

We shall show its coefficient is 1 . Note that the leading term is determined by

$$
t_{k+i}=R_{i}, t_{j}=0, \forall i \in[0, l], j \notin[k, k+l] .
$$


In this case, we have $\prod_{u \in \mathbb{Z}} \overline{\left[\begin{array}{c}a_{h u}+t_{u} \\ t_{u}\end{array}\right]}=1$ and

$$
\beta(t)=\sum_{j \geqslant u}\left(t_{j}-a_{h+1, j}\right) t_{u}=\sum_{i=0}^{l} \sum_{j>k+i}\left(t_{j}-a_{h+1, j}\right) R_{i}=0 .
$$

This shows (1). Part (2) can be proved similarly.

A product of standard basis elements $\left[G_{1}\right] *\left[G_{2}\right] * \cdots *\left[G_{m}\right]$ in $\mathbf{S}_{n, d}$ is called an aperiodic monomial if for each $i$, either $G_{i}-R E^{j, j+1}$ or $G_{i}-R E^{j+1, j}$ is diagonal for some $R \in \mathbb{N}$ and $j \in \mathbb{Z}$. The following proposition is a missing piece in the affine generalization of [BLM90], corresponding to Proposition 3.9 in the loc. cit. We refer to [DD05] and the references therein for early treatments using Ringel-Hall algebras and generic extension.

Proposition 2.2.3. For any $A \in \Theta_{n, d}^{a p}$, there exists (and we shall fix) an aperiodic monomial $\zeta_{A}^{\mathfrak{a}}$ such that $\zeta_{A}^{\mathfrak{a}}=[A]+$ lower terms. Moreover, the set $\left\{\zeta_{A}^{\mathfrak{a}} \mid A \in \Theta_{n, d}^{a p}\right\}$ is a basis for $\mathbf{U}_{n, d}$.

Proof. Recall [Lu99] that $\left\{\mathfrak{a}\{A\}_{d} \mid A \in \Theta_{n, d}^{a p}\right\}$ forms a canonical basis for $\mathbf{U}_{n, d}$. Assuming the first statement on the existence of such $\zeta_{A}^{\mathfrak{a}}$, we then have $\zeta_{A}^{\mathfrak{a}}={ }^{\mathfrak{a}}\{A\}_{d}+$ lower terms in $\mathbf{U}_{n, d}$, and hence $\left\{\zeta_{A}^{\mathfrak{a}} \mid A \in \Theta_{n, d}^{a p}\right\}$ forms a basis for $\mathbf{U}_{n, d}$.

It remains to prove the existence of such an aperiodic monomial $\zeta_{A}^{\mathfrak{a}}$. Let us fix some notations. Given a matrix $A=\left(a_{i j}\right) \in \Theta_{n, d}$, we define a matrix

$$
f_{k ; s, t}(A)=A-\sum_{s \leqslant j \leqslant t} a_{k-1, j}\left(E^{k-1, j}-E^{k, j}\right) \in \Theta_{n, d} .
$$

Let $\Psi(A)=\sum_{i \in[1, n]}|j-i| a_{i j}$. It is clear that $\Psi\left(f_{k ; s, t}(A)\right) \leqslant \Psi(A)$ for all $k, s$ and $t$ with $k \leqslant s \leqslant t$, where the equality holds if and only if

$$
a_{k-1, j}=0, \forall s \leqslant j \leqslant t .
$$

We are now ready to prove the existence of such an aperiodic monomial $\zeta_{A}^{\mathfrak{a}}$ by induction on $\Psi(A)$. If $\Psi(A)=0$, then $A$ is a diagonal matrix, and $\zeta_{A}^{\mathfrak{a}}=[A]$.

We now assume that $\Psi(A) \geqslant 1$ and that the existence of such $\zeta_{A^{\prime}}^{\mathfrak{a}}$ for all aperiodic matrices $A^{\prime}$ with $\Psi\left(A^{\prime}\right)<\Psi(A)$. Set $m=\min \left\{l \in \mathbb{N} \mid a_{i j}=0\right.$ for all $\left.|i-j|>l\right\}$. If there exists $k \in \mathbb{Z}$ such that $a_{k, k+m}=0$ and $a_{k-1, k-1+m} \neq 0$. By (2.2.11), we have $\Psi\left(f_{k ; s, t}(A)\right)<\Psi(A)$ for all $k \leqslant s \leqslant t$.

Let $u=\max \left\{s \leqslant k+m-1 \mid f_{k ; s, k+m-1}(A)\right.$ is aperiodic $\}$. We have $a_{k l}=0$ for all $l>u$. (Otherwise, there exists $j>u$ such that $a_{k j} \neq 0$. Then $f_{k ; j, k+m-1}(A)$ is aperiodic, which contradicts with the definition of $u$.) By Lemma 2.2.2 (1), we have

$$
[B] *\left[f_{k, u, k+m-1}(A)\right]=[A]+\text { lower terms, }
$$

where $B$ is the matrix such that $\operatorname{co}(B)=\operatorname{ro}\left(f_{k, u, k+m-1}(A)\right)$ and $B-\sum_{l=u}^{k+m-1} a_{k-1, l} E^{k-1, k}$ is diagonal.

If there exists $k \in \mathbb{Z}$ such that $a_{k, k-m} \neq 0$ and $a_{k-1, k-1-m}=0$, we can prove a statement similar to (2.2.12) by using Lemma 2.2.2(2). By induction on $\Psi(A)$, the existence of $\zeta_{A}^{\mathfrak{a}}$ follows. 
Example 2.2.4. Let $n=2$. Let $A$ be a lower triangular matrix whose nonzero entries are located at $(5,5),(6, j)(\bmod 2)$, for $2 \leqslant j \leqslant 6$, which are

$$
a_{55}=1, a_{62}=2, a_{63}=3, a_{64}=2, a_{65}=1, a_{66}=2 .
$$

Let $A^{\prime}=\left(a_{i j}^{\prime}\right)$ be the lower triangular matrix whose nonzero entries are specified by

$$
a_{52}^{\prime}=2, a_{53}^{\prime}=3, a_{54}^{\prime}=2, a_{55}^{\prime}=2, a_{66}^{\prime}=2 .
$$

Applying the algorithm in the proof, $\mathbf{E}_{1}^{(8)} *\left[A^{\prime}\right]=[A]+$ lower terms. Inductively, we have

$$
\mathbf{E}_{1}^{(8)} * \mathbf{E}_{0}^{(7)} * \mathbf{E}_{1}^{(5)} * \mathbf{E}_{0}^{(2)} * 1_{\operatorname{co}(A)}=[A]+\text { lower terms. }
$$

\subsection{AlgeBras $\mathbf{U}_{n}$ AND $\dot{\mathbf{U}}_{n}$}

Recall a transfer map $\phi_{d, d-n}: \mathbf{U}_{n, d} \rightarrow \mathbf{U}_{n, d-n}$ was introduced in [Lu00] by sending the generators $\mathbf{E}_{i}^{(R)}, \mathbf{F}_{i}^{(R)}$ and $\mathbf{K}_{i}^{ \pm 1}$ to the respective generators. Let us define a partial order $\leqslant_{n}$ on $\mathbb{N}$ by declaring that

$$
a \leqslant_{n} b \text { iff } b-a=p n \quad \text { for some } p \geqslant 0 .
$$

Then $\left\{\left(\mathbf{U}_{n, d}, \phi_{d, d-n}\right)\right\}_{d \in \mathbb{N}}$ form a projective system over the poset $\left(\mathbb{N}, \leqslant_{n}\right)$. We shall consider its projective limit:

$$
\mathbf{U}_{n, \infty} \equiv \lim _{d} \mathbf{U}_{n, d}=\left\{x \equiv\left(x_{d}\right)_{d \in \mathbb{N}} \in \prod_{d \in \mathbb{N}} \mathbf{U}_{n, d} \mid \phi_{d, d-n}\left(x_{d}\right)=x_{d-n} \quad \forall d\right\} .
$$

The bar involution on $\mathbf{U}_{n, d}$ induces a bar involution ${ }^{-}: \mathbf{U}_{n, \infty} \rightarrow \mathbf{U}_{n, \infty}$, since it commutes with the transfer map [Lu00]. Similarly, we have an integral version: $\mathbf{U}_{n, \infty ; \mathcal{A}}=\lim _{d} \mathbf{U}_{n, d ; \mathcal{A}}$. Since $\mathbb{Q}(v) \otimes_{\mathcal{A}} \mathbf{U}_{n, d ; \mathcal{A}}=\mathbf{U}_{n, d}$ for all $d$, we have $\mathbb{Q}(v) \otimes_{\mathcal{A}} \mathbf{U}_{n, \infty ; \mathcal{A}}=\mathbf{U}_{n, \infty}$.

As we deal with all $d \in \mathbb{N}$ simultaneously, we will write

$$
1_{\lambda, d}, \mathbf{E}_{i, d}, \mathbf{F}_{i, d}, \mathbf{K}_{i, d}^{ \pm 1}, \quad \forall 1 \leqslant i \leqslant n
$$

for the generators in $\mathbf{U}_{n, d}$, which are denoted without $d$ in the subscript previously. Since the transfer map sends generators $\mathbf{E}_{i, d}, \mathbf{F}_{i, d}$ and $\mathbf{K}_{i, d}^{ \pm 1}$ to the respective generators, we can define elements $\mathbf{E}_{i}, \mathbf{F}_{i}$ and $\mathbf{K}_{i}^{ \pm 1}$ for all $1 \leqslant i \leqslant n$ in $\mathbf{U}_{n, \infty}$ by declaring that their $d$-th component is $\mathbf{E}_{i, d}, \mathbf{F}_{i, d}$ and $\mathbf{K}_{i, d}^{ \pm 1}$, respectively. (Similarly, we can define the $a$-th divided power $\mathbf{E}_{i}^{(a)}$ and $\mathbf{F}_{i}^{(a)}$.)

Definition 2.3.1. Let $\mathbf{U}_{n}$ be the subalgebra of $\mathbf{U}_{n, \infty}$ generated by $\mathbf{E}_{i}, \mathbf{F}_{i}$ and $\mathbf{K}_{i}^{ \pm 1}$ for all $1 \leqslant i \leqslant n$.

Clearly, the restriction of the natural projection $\phi_{d}: \mathbf{U}_{n, \infty} \rightarrow \mathbf{U}_{n, d}$ gives us a surjective algebra homomorphism:

$$
\phi_{d}: \mathbf{U}_{n} \longrightarrow \mathbf{U}_{n, d}
$$

We set

$$
\mathbb{Z}_{n}=\left\{\lambda=\left(\lambda_{i}\right)_{i \in \mathbb{Z}} \mid \lambda_{i} \in \mathbb{Z}, \lambda_{i}=\lambda_{i+n}, \forall i\right\} .
$$

We define an equivalence relation $\sim$ on $\mathbb{Z}_{n}$ by

$$
\lambda \sim \mu \Leftrightarrow \lambda-\mu=(\ldots, p, p, p, \ldots), \text { for some } p \in \mathbb{Z} \text {. }
$$


Let $\mathbb{Z}_{n} / \sim$ be the set of equivalence classes and $\bar{\lambda}$ be the equivalence class of $\lambda$. Let

$$
\mathbb{X}=\mathbb{Z}_{n} / \sim, \quad \mathbb{Y}=\left\{\nu \in \mathbb{Z}_{n} \mid \sum_{1 \leqslant i \leqslant n} \nu_{i}=0\right\}
$$

Then the standard dot product on $\mathbb{Z}_{n}$ induces a pairing $\cdot: \mathbb{Y} \times \mathbb{X} \longrightarrow \mathbb{Z}$. Set $I=\{1, \ldots, n\}$. We define two injective maps $I \rightarrow \mathbb{Y}, \quad I \rightarrow \mathbb{X}$, by letting

$$
i \mapsto-\epsilon_{i}+\epsilon_{i+1}, i \mapsto-\bar{\epsilon}_{i}+\bar{\epsilon}_{i+1}, \quad \forall 1 \leqslant i \leqslant n,
$$

respectively, where $\epsilon_{i}$ is the $i$-th standard basis element in $\mathbb{Z}_{n}$, that is $\left(\epsilon_{i}\right)_{j}=\delta_{\bar{i}, \bar{j}}$. We thus obtain a root datum of affine type $A_{n-1}$ in [Lu93, 2.2].

For each $\bar{\lambda} \in \mathbb{X}$, we define an element $1_{\bar{\lambda}}$ in $\mathbf{U}_{n, \infty}$ by setting $\left(1_{\bar{\lambda}}\right)_{d}=0$ unless $|\lambda|:=$ $\sum_{1 \leqslant i \leqslant n} \lambda_{i}=d \bmod n$, and in which case $\left(1_{\bar{\lambda}}\right)_{d}=1_{\mu, d}$ where $\mu \in \bar{\lambda}$ and $|\mu|=d$. We define $\dot{\mathbf{U}}_{n}$ to be the $\mathbf{U}_{n}$-bimodule in $\mathbf{U}_{n, \infty}$ generated by $1_{\bar{\lambda}}$ for all $\bar{\lambda} \in \mathbb{X}$. It is clear then that $\dot{\mathbf{U}}_{n}$ is naturally a subalgebra in $\mathbf{U}_{n, \infty}$. The algebra $\dot{\mathbf{U}}_{n}$ admits a decomposition

$$
\dot{\mathbf{U}}_{n}=\bigoplus_{\bar{\lambda} \in \mathbb{X}} \mathbf{U}_{n} 1_{\bar{\lambda}}=\bigoplus_{\bar{\mu}, \bar{\lambda} \in \mathbb{X}} \bar{\mu}\left(\dot{\mathbf{U}}_{n}\right)_{\bar{\lambda}},
$$

where ${ }_{\bar{\mu}}\left(\dot{\mathbf{U}}_{n}\right)_{\bar{\lambda}}=1_{\bar{\mu}} \dot{\mathbf{U}}_{n} 1_{\bar{\lambda}}$.

Let $\mathbf{U}\left(\widehat{\mathfrak{s l}}_{n}\right)$ be the affine quantum group of type $A_{n-1}$ (of level zero) attached to the above root datum. Let $\dot{\mathbf{U}}\left(\widehat{\mathfrak{s l}}_{n}\right)$ be its modified form. The following result is due to Lusztig [Lu00, Proposition 3.5] (more precisely, the first one was explicitly written down therein, while the second one is folklore as it follows in the same way as in the finite type $A$ case [BLM90].)

Proposition 2.3.2. We have the algebra isomorphisms $\dot{\mathbf{U}}\left(\hat{\mathfrak{s l}}_{n}\right) \simeq \dot{\mathbf{U}}_{n}$, and $\mathbf{U}\left(\hat{\mathfrak{s l}}_{n}\right) \simeq \mathbf{U}_{n}$.

Proof. We regard $\dot{\mathbf{U}}_{n}$ as the left modules of $\mathbf{U}_{n}$ and $\left.\mathbf{U}(\widehat{\mathfrak{s l}})_{n}\right)$. Then we have two algebra homomorphisms $\mathbf{U}_{n} \rightarrow \operatorname{End}\left(\dot{\mathbf{U}}_{n}\right)$, and $\mathbf{U}\left(\hat{\mathfrak{s l}}_{n}\right) \rightarrow \operatorname{End}\left(\dot{\mathbf{U}}_{n}\right)$. Both maps are injective and have obviously the same image, so they must be isomorphic. (In short, $\mathbf{U}_{n}$ and $\mathbf{U}\left(\widehat{\mathfrak{s l}}_{n}\right)$ act faithfully on $\dot{\mathbf{U}}_{n}$.)

Therefore the geometric pair $\left(\mathbf{U}_{n}, \dot{\mathbf{U}}_{n}\right)$ is identified with the algebraic pair $\left(\mathbf{U}\left(\widehat{\mathfrak{s l}}_{n}\right), \dot{\mathbf{U}}\left(\widehat{\mathfrak{s l}}_{n}\right)\right)$. 


\section{CHAPTER 3}

\section{Lattice presentation of affine flag varieties of type $C$}

We present lattice models for the variety $\mathcal{Y}^{\mathfrak{c}}$ of affine complete flags and a variety $\mathcal{X}_{n, d}^{\mathfrak{c}}$ of $n$-step flags in an $F$-vector space $V$ of affine type $C$, for $n$ even. Then we classify the $\operatorname{Sp}_{F}(V)$-orbits on $\mathcal{X}_{n, d}^{\mathfrak{c}} \times \mathcal{X}_{n, d}^{\mathfrak{c}}, \mathcal{X}_{n, d}^{\mathfrak{c}} \times \mathcal{Y}^{\mathfrak{c}}$, and $\mathcal{Y}^{\mathfrak{c}} \times \mathcal{Y}^{\mathfrak{c}}$.

\subsection{Affine COMPlete Flag VARIETIES OF TYPE $C$}

Recall $k$ is a finite field of odd $q$ elements, $F=k((\varepsilon))$ is the field of formal Laurent series over $k$, and $\mathfrak{o}=k[[\varepsilon]]$ the ring of formal power series. Let $d$ be a positive integer. Let

$$
J=\left(\begin{array}{ccccc}
0 & 0 & \cdots & 0 & 1 \\
0 & 0 & \cdots & 1 & 0 \\
\cdot & . & \cdots & . & . \\
1 & 0 & \cdots & 0 & 0
\end{array}\right)_{d \times d}, \quad M=M_{2 d}=\left(\begin{array}{cc}
0 & J \\
-J & 0
\end{array}\right)
$$

Let $V=F^{2 d}$ be a symplectic vector space over $F$ with a symplectic form $():, V \times V \longrightarrow F$ specified by $M$. Let ${ }^{t} g$ be the transpose of a matrix $g$. We define the symplectic group with coefficients in $F$

$$
\operatorname{Sp}_{F}(2 d)=\left\{g \in \mathrm{GL}_{F}(2 d) \mid g=M^{t} g^{-1} M^{-1}\right\} .
$$

We also define $\operatorname{Sp}_{\mathfrak{o}}(2 d)$ and $\operatorname{Sp}_{k}(2 d)$ similarly. By our choice of $M$, we see that $P \cap \operatorname{Sp}_{F}(2 d)$ is parahoric if $P$ is parahoric in $\mathrm{GL}_{F}(2 d)$. In particular, $\mathbf{I}^{\mathfrak{c}}=\mathbf{I}^{\mathfrak{a}} \cap \operatorname{Sp}_{F}(2 d)$ is Iwahori and it is the stabilizer of the standard lattice chain $\mathrm{E}$ in $\operatorname{Sp}_{F}(V)$. Therefore, we have the bijection

$$
\operatorname{Sp}_{F}(2 d) / \mathbf{I}^{\mathfrak{c}} \simeq \operatorname{Sp}_{F}(2 d) \cdot \mathrm{E} \equiv \tilde{\mathcal{Y}}^{\mathfrak{c}}
$$

So the lattice presentation of affine flag variety of type $C$ is reduced to a description of $\tilde{\mathcal{Y}}^{c}$. For any lattice $\mathcal{L}$ of $V$, we set

$$
\mathcal{L}^{\#}=\{v \in V \mid(v, \mathcal{L}) \subset \mathfrak{o}\} .
$$

Then the o-module $\mathcal{L}^{\#}$ is again a lattice of $V$ and $\left(\mathcal{L}^{\#}\right)^{\#}=\mathcal{L}$. We shall use freely the following properties: for any two lattices $\mathcal{L}$ and $\mathcal{M}$

$$
(\mathcal{L}+\mathcal{M})^{\#}=\mathcal{L}^{\#} \cap \mathcal{M}^{\#}, \quad(\mathcal{L} \cap \mathcal{M})^{\#}=\mathcal{L}^{\#}+\mathcal{M}^{\#}
$$

Following Sage Sa99, we call a lattice alternating if $\mathcal{L} \subseteq \mathcal{L}^{\#}$ or $\mathcal{L} \supseteq \mathcal{L}^{\#}$. An alternating lattice $\mathcal{L}$ is called sympletic if $\mathcal{L}$ or $\mathcal{L}^{\#}$ is homothetic to a lattice $\Lambda$, i.e., $\mathcal{L}$ or $\mathcal{L}^{\#}$ is equal to $\varepsilon^{a} \Lambda$ for some $a \in \mathbb{Z}$, such that

$$
\varepsilon \Lambda \subseteq \Lambda^{\#} \subseteq \Lambda
$$

Clearly $\mathrm{E}_{z}$ are symplectic for $z \in \mathbb{Z}$. The following proposition can be found in [H99, Sa99, Lu03. 
Proposition 3.1.1. The set $\tilde{\mathcal{Y}}^{\mathfrak{c}}$ is the set of all collections $L=\left(L_{z}\right)_{z \in \mathbb{Z}}$ of symplectic lattices in $V$ subject to the following conditions:

$$
L_{z} \subset L_{z+1}, \quad \operatorname{dim}_{k} L_{z+1} / L_{z}=1, \quad L_{z}=\varepsilon L_{z+2 d}, \quad L_{z}^{\#}=L_{-z} \quad(\forall z \in \mathbb{Z}) .
$$

For our purpose later, we define a variant of the set $\tilde{\mathcal{Y}}^{\mathfrak{c}}$ as follows. Let $\mathcal{Y}^{\mathfrak{c}}$ be the set of all chains $L=\left(L_{z} \mid z \in \mathbb{Z}\right)$ of symplectic lattices subject to the following conditions:

$$
\begin{aligned}
& \operatorname{dim}_{k} L_{z+1} / L_{z}= \begin{cases}0, & \text { if } z \equiv-1, d \bmod 2 d+2, \\
1, & \text { otherwise; }\end{cases} \\
& L_{z} \subset L_{z+1}, \quad L_{z}=\varepsilon L_{z+2 d+2}, \quad L_{z}^{\#}=L_{-z-1} \quad(\forall z \in \mathbb{Z}) .
\end{aligned}
$$

Clearly, we have a natural bijection: $\tilde{\mathcal{Y}}^{\mathfrak{c}} \simeq \mathcal{Y}^{\mathfrak{c}}$.

Via the identification $\operatorname{Sp}_{F}(2 d) / \mathbf{I}^{\mathfrak{c}} \cong \mathcal{Y}^{\mathfrak{c}}$, there is a left action of $\operatorname{Sp}_{F}(2 d)$ on $\mathcal{Y}^{\mathfrak{c}}$ which is transitive. Let $\operatorname{Sp}_{F}(2 d)$ act on the product $\mathcal{Y}^{\mathfrak{c}} \times \mathcal{Y}^{\mathfrak{c}}$ diagonally. We shall describe the $\operatorname{Sp}_{F}(2 d)$-orbits in $\mathcal{Y}^{\mathfrak{c}} \times \mathcal{Y}^{\mathfrak{c}}$.

Recall a set $\Theta_{n \mid n^{\prime}, d}$ was defined in (2.2.1) for any positive integers $d, n, n^{\prime}$. Let ${ }^{\mathfrak{c}} \Sigma_{d}$ be the following subset of $\Theta_{2 d+2 \mid 2 d+2,2 d}$ of matrices with entries being 0 or 1:

$$
\begin{aligned}
{ }^{c} \Sigma_{d}=\left\{A \in \operatorname{Mat}_{\mathbb{Z} \times \mathbb{Z}}(\{0,1\}) \mid a_{-i,-j}=a_{i j}=a_{i+2 d+2, j+2 d+2}(\forall i, j \in \mathbb{Z}),\right. \\
\text { the } 0 \text { th and }(d+1) \text { st rows/columns are zero, } \\
\quad \exists \text { exactly one nonzero entry per row/column } i \in[0,2 d+1] \backslash\{0, d+1\}\} .
\end{aligned}
$$

We define a map from the set of $\operatorname{Sp}_{F}(2 d)$-orbits in $\mathcal{Y}^{\mathfrak{c}} \times \mathcal{Y}^{\mathfrak{c}}$ to ${ }^{\mathfrak{c}} \Sigma_{d}$ :

$$
\varphi: \operatorname{Sp}_{F}(2 d) \backslash \mathcal{Y}^{\mathfrak{c}} \times \mathcal{Y}^{\mathfrak{c}} \longrightarrow{ }^{\mathfrak{c}} \Sigma_{d}
$$

by sending the orbit $\operatorname{Sp}_{F}(2 d) .\left(L, L^{\prime}\right)$ to $A=\left(a_{i j}\right)_{i, j \in \mathbb{Z}}$ where

$$
a_{i j}=\operatorname{dim}_{k} \frac{L_{i-1}+L_{i} \cap L_{j}^{\prime}}{L_{i-1}+L_{i} \cap L_{j-1}^{\prime}} .
$$

By the definition of $a_{i j}$, we have

$$
\begin{aligned}
a_{-i,-j} & =\operatorname{dim}_{k} \frac{L_{-i-1}+L_{-i} \cap L_{-j}^{\prime}}{L_{-i-1}+L_{-i} \cap L_{-j-1}^{\prime}}=\operatorname{dim}_{k} \frac{L_{i}^{\#}+L_{i-1}^{\#} \cap L_{j-1}^{\prime \#}}{L_{i}^{\#}+L_{i-1}^{\#} \cap L_{j}^{\prime \#}} \\
& =\operatorname{dim}_{k} \frac{\left(L_{i} \cap\left(L_{i-1}+L_{j-1}^{\prime}\right)\right)^{\#}}{\left(L_{i} \cap\left(L_{i-1}+L_{j}^{\prime}\right)\right)^{\#}}=\operatorname{dim}_{k} \frac{L_{i} \cap\left(L_{i-1}+L_{j}^{\prime}\right)}{L_{i} \cap\left(L_{i-1}+L_{j-1}^{\prime}\right)} \\
& =\operatorname{dim}_{k} \frac{L_{i-1}+L_{i} \cap L_{j}^{\prime}}{L_{i-1}+L_{i} \cap L_{j-1}^{\prime}}=a_{i j} .
\end{aligned}
$$

So the map $\varphi$ is well defined. The following proposition can be found in [H99], see also [Lu99].

Proposition 3.1.2. [H99, Proposition 2.6] Let $A=\left(a_{i j}\right)_{i, j \in \mathbb{Z}}$ be the associated matrix of $\left(L, L^{\prime}\right)$ under $\varphi$. Then we can decompose $V$ into $V=\oplus_{i, j \in \mathbb{Z}} V_{i j}$ as $k$-vector spaces satisfying that $\operatorname{dim}_{k} V_{i j}=a_{i j}$,

$$
L_{i}=\bigoplus_{k, l \in \mathbb{Z}: k \leqslant i} V_{k l}, \quad L_{j}^{\prime}=\bigoplus_{k, l \in \mathbb{Z}: l \leqslant j} V_{k l}, \quad \forall i, j \in \mathbb{Z}
$$


Moreover, there exists a basis $\left\{e_{i j}^{m} \mid 1 \leqslant m \leqslant a_{i j}\right\}$ of $V_{i j}$ such that

$$
\begin{aligned}
e_{i, j}^{m} & =\varepsilon e_{i+2 d+2, j+2 d+2}^{m}, \quad \forall i, j \in \mathbb{Z}, 1 \leqslant m \leqslant a_{i j}, \\
\left(e_{i j}^{m}, e_{k l}^{m^{\prime}}\right) & =-\left(e_{k l}^{m^{\prime}}, e_{i j}^{m}\right), \quad \forall i, j, k, l \in \mathbb{Z}, 1 \leqslant m \leqslant a_{i j}, 1 \leqslant m^{\prime} \leqslant a_{k l}, \\
\left(e_{i j}^{m}, e_{k l}^{m^{\prime}}\right) & =\varepsilon\left(e_{i j}^{m}, e_{k+(2 d+2), l+(2 d+2)}^{m^{\prime}}\right), \quad \forall i, j, k, l \in \mathbb{Z}, 1 \leqslant m \leqslant a_{i j}, 1 \leqslant m^{\prime} \leqslant a_{k l}, \\
\left(e_{i j}^{m}, e_{k l}^{m^{\prime}}\right) & =\delta_{m, 1} \delta_{m^{\prime}, 1} \varepsilon^{-2}, \quad \forall 1 \leqslant i<k \leqslant 2 d+2, i+k=2 d+2, j+l=2 d+2 .
\end{aligned}
$$

From the above proposition, we have the Iwahori-Bruhat decomposition for the group $\operatorname{Sp}_{F}(V)$.

Proposition 3.1.3. The map $\varphi: \operatorname{Sp}_{F}(2 d) \backslash \mathcal{Y}^{\mathfrak{c}} \times \mathcal{Y}^{\mathfrak{c}} \longrightarrow{ }^{\mathfrak{c}} \Sigma_{d}$ in 3.1.7) is a bijection.

Proof. By Proposition 3.1.2, $\varphi$ is clearly surjective. Assume now that the associated matrix of two pairs $\left(L, L^{\prime}\right)$ and $\left(\tilde{L}, \tilde{L}^{\prime}\right)$ of symplectic lattice chains is the same matrix, say $A$. By Proposition 3.1 .2 , we can find bases $\left\{e_{i j}^{m}\right\}$ and $\left\{f_{i j}^{m}\right\}$ for the pairs $\left(L, L^{\prime}\right)$ and $\left(\tilde{L}, \tilde{L}^{\prime}\right)$, respectively, subject to the conditions (3.1.8) and (3.1.9). We define a map $g: V \rightarrow V$ by sending $e_{i j}^{m}$ to $f_{i j}^{m}$ for all $i, j \in \mathbb{Z}$ and $1 \leqslant m \leqslant a_{i j}$. Then we have $g \in \operatorname{Sp}_{F}(2 d)$ and $g\left(L, L^{\prime}\right)=\left(\tilde{L}, \tilde{L}^{\prime}\right)$. So $\varphi$ is injective. The proposition is proved.

\subsection{Affine partial Flag varieties of type $C$}

Now we fix an even positive integer

$$
n=2 r+2, \quad \text { for some } r \in \mathbb{N} \text {. }
$$

Let $\mathcal{X}_{n, d}^{\mathfrak{c}}$ be the set of all chains $L=\left(L_{z}\right)_{z \in \mathbb{Z}}$ of symplectic lattices in $V$ subject to the following conditions:

$$
L_{z} \subset L_{z+1}, \quad L_{z}=\varepsilon L_{z+n}, \quad L_{z}^{\#}=L_{-z-1} \quad(\forall z \in \mathbb{Z}) .
$$

Remark 3.2.1. The shift by -1 in the condition $L_{z}^{\#}=L_{-z-1}$ in definition of $\mathcal{X}_{n, d}^{\mathfrak{c}}$ (see (3.2.1) ) allows the valuation at $L_{0}$ to vary. In contrast the valuation at $L_{0}$ is always zero in the case of $\tilde{\mathcal{Y}}^{c}$.

The group $\operatorname{Sp}_{F}(2 d)$ acts transitively from the left on $\mathcal{X}_{n, d}^{\mathfrak{c}}$ in a standard way. Let $\operatorname{Sp}_{F}(2 d)$ act diagonally on the products $\mathcal{X}_{n, d}^{\mathfrak{c}} \times \mathcal{X}_{n, d}^{\mathfrak{c}}$ and $\mathcal{X}_{n, d}^{\mathfrak{c}} \times \mathcal{Y}^{\mathfrak{c}}$. Let $\Pi_{n, d}$ be the subset of $\Theta_{n \mid 2 d+2,2 d}$ (for $\Theta_{n \mid n^{\prime}, d}$ see $(\underline{2.2 .1})$ ), which consists of all matrices $A=\left(a_{i j}\right) \in \operatorname{Mat}_{\mathbb{Z} \times \mathbb{Z}}(\mathbb{N})$ such that

$$
a_{-i,-j}=a_{i j}=a_{i+n, j+2 d+2}(\forall i, j \in \mathbb{Z}), \quad \sum_{l \in \mathbb{Z}} a_{l j}= \begin{cases}0, & \forall j \equiv 0, d+1(\bmod 2 d+2) \\ 1, & \text { otherwise. }\end{cases}
$$

Similar to (3.1.7), we have a map

$$
\operatorname{Sp}_{F}(2 d) \backslash \mathcal{X}_{n, d}^{\mathfrak{c}} \times \mathcal{Y}^{\mathfrak{c}} \longrightarrow \Pi_{n, d}
$$

More generally, let ${ }^{\mathfrak{c}} \Xi_{n, d}$ be the subset of $\Theta_{n, 2 d}$ given by

$$
\begin{aligned}
{ }^{\mathfrak{c}} \Xi_{n, d}=\left\{\left(a_{i j}\right) \in \operatorname{Mat}_{\mathbb{Z} \times \mathbb{Z}}(\mathbb{N}) \mid\right. & a_{-i,-j}=a_{i j}=a_{i+n, j+n}, \quad(\forall i, j), \\
& \left.\sum_{1 \leqslant i \leqslant n} \sum_{j \in \mathbb{Z}} a_{i j}=2 d, a_{00}, a_{r+1, r+1} \in 2 \mathbb{Z}\right\} .
\end{aligned}
$$


Similar to (3.1.7) again, we have a map

$$
\operatorname{Sp}_{F}(2 d) \backslash \mathcal{X}_{n, d}^{\mathfrak{c}} \times \mathcal{X}_{n, d}^{\mathfrak{c}} \longrightarrow{ }^{\mathfrak{c}} \Xi_{n, d}
$$

Proposition 3.2.2. The maps in (3.2.3) and (3.2.5) are bijective.

Proof. Note that the first bijection (3.2.3) is a special case of the second bijection (3.2.5). So we only need to prove the second bijection, and we shall deduce it from Proposition 3.1 .3 as follows. For a given matrix $A \in{ }^{\mathfrak{c}} \Xi_{n, d}$, we can delete all its zero rows and zero columns. Let us denote the resulting matrix by $\operatorname{dlt}(A)$, which is essentially determined by the stripe $[1, a] \times \mathbb{Z}$ of $\operatorname{dlt}(A)$ for some $a \leqslant 2 d$. It is then possible to find a (nonunique) matrix $w \in{ }^{\mathfrak{c}} \Sigma_{d}$ (see (3.1.6) ) such that $A$ can be obtained from $w$ by adding consecutive rows between $[1,2 d+2]$. Now pick a representative, say $\left(L, L^{\prime}\right)$, in the orbit $\mathcal{O}_{w}$. We can construct a pair of partial flags by removing subspaces in $L$ and $L^{\prime}$ corresponding to the summations of consecutive rows, whose associated matrix is $\operatorname{dlt}(A)$. This shows that the map (3.2.5) is surjective.

Now if there are two pairs, say $x, y$, of flags whose associated matrix is $A$, we fix a matrix $w \in{ }^{\mathfrak{c}} \Sigma$ such that it can be merged to $A$, and use the above process to find two pairs, $x^{\prime}, y^{\prime}$ of flags in $\mathcal{O}_{w}$ such that they can reach $x, y$, respectively, by throwing away certain steps. Moreover, $w$ can be chosen to be the one obtained from $A$ by blowing up the entries in $A$ of value strictly greater than 1 to an identity matrix locally. For example, if $a_{i j}=2$ and $x=\left(L, L^{\prime}\right)$, we can find a vector $u \in L_{i} \cap L_{j}^{\prime}-\left(L_{i-1} \cap L_{j}^{\prime}+L_{i} \cap L_{j-1}^{\prime}\right)$ such that $L_{i-1}+\mathfrak{o} u$ and $L_{j-1}^{\prime}+\mathfrak{o} u$ are symplectic lattices. We expand $L$ by plugging the lattice $L_{i}+\mathfrak{o} u$ in between $L_{i}$ and $L_{i+1}$. Similarly, we can expand $L^{\prime}$. Then the matrix of the resulting pair will be the one by blowing up $A$ at $(i, j)$ to be a $2 \times 2$ identity matrix locally. By repeating the above process, we have the desired pair $x^{\prime}$ for $x$ whose matrix is $w$. Since $x^{\prime}$ and $y^{\prime}$ are in the same orbit, there is a $g \in \operatorname{Sp}_{F}(2 d)$ such that $g \cdot x^{\prime}=y^{\prime}$, which induces that $g \cdot x=y$. So $x$ and $y$ are in the same orbit. Therefore the map (3.2.5) is injective, and hence a bijection. The proposition is proved.

\subsection{LOCAL PROPERTY AT $L_{0}$}

Lemma 3.3.1. Let $L=\left(L_{z}\right)_{z \in \mathbb{Z}} \in \mathcal{X}_{n, d}^{\mathfrak{c}}$. We have

$$
v\left(L_{r}\right) \in[-d, 0], \quad v\left(L_{r+1}\right) \in[-2 d,-d], \quad \text { and } \quad v\left(L_{r}\right)+v\left(L_{r+1}\right)=-2 d .
$$

Proof. Note that the valuation of $L_{0}$ is non-positive because $L_{-1}=L_{0}^{\#}$. So the valuation $v\left(L_{r}\right) \leqslant 0$.

By definition, for any lattice $\mathcal{L}$, we have

$$
v\left(\mathcal{L}^{\#}\right)=-v(\mathcal{L}), \quad v(\varepsilon \mathcal{L})=2 d+v(\mathcal{L})
$$

So we have

$$
v\left(L_{r+1}\right)=v\left(\varepsilon^{-1} L_{-(r+1)}\right)=-2 d+v\left(L_{-(r+1)}\right)=-2 d+v\left(L_{r}^{\#}\right)=-2 d-v\left(L_{r}\right) .
$$

Since $v\left(L_{r+1}\right) \leqslant v\left(L_{r}\right)$, we have $v\left(L_{r}\right) \geqslant-d$, and $-d \geqslant v\left(L_{r+1}\right) \geqslant-2 d$.

More generally, we have $v\left(L_{i}\right) \in[-d, 0], v\left(L_{i}\right)+v\left(L_{n-1-i}\right)=-2 d, \quad \forall i \in[0, r]$, by the same type of argument above. But we do not need this general fact. 
Then, we can find a 'maximal isotropic' lattice $L_{r^{\dagger}}$ isometric to

$$
\mathrm{七}_{d}=\left[e_{d+1}, \ldots, e_{2 d}, \varepsilon^{-1} e_{1}, \ldots, \varepsilon^{-1} e_{d}\right]_{\mathfrak{o}},
$$

such that

$$
L_{r} \subseteq L_{r^{\dagger}} \subseteq L_{r+1}
$$

Here the basis $\left\{e_{i} \mid 1 \leqslant i \leqslant 2 d\right\}$ is chosen such that the associated matrix of the symplectic form on $V$ is given by (3.1.1). The lattice $\mathrm{E}_{d}$ satisfies the following properties:

$$
\left(\mathrm{E}_{d}, \mathrm{E}_{d}\right)=\varepsilon^{-1} \mathfrak{o}, \quad\left(\mathrm{E}_{d}, \varepsilon \mathrm{E}_{d}\right)=\mathfrak{o}, \quad\left(\varepsilon \mathrm{E}_{d}, \mathrm{E}_{d}\right)=\mathfrak{o} .
$$

So the map

$$
\begin{aligned}
(-,-)_{r^{\dagger}} & : L_{r^{\dagger}} / \varepsilon L_{r^{\dagger}} \times L_{r^{\dagger}} / \varepsilon L_{r^{\dagger}} \rightarrow k \\
(\bar{x}, \bar{y})_{r^{\dagger}} & =\left.\mathrm{ev}\right|_{\varepsilon=0} \varepsilon(x, y)
\end{aligned}
$$

is a non-degenerate symplectic form on $L_{r^{\dagger}} / \varepsilon L_{r^{\dagger}} \simeq k^{2 d}$.

Moreover, $L_{-1} / \varepsilon L_{r^{\dagger}}$ and $L_{0} / \varepsilon L_{r^{\dagger}}$ are orthogonal complements to each other with respect to the above form $(-,-)_{r^{\dagger}}$ on $L_{r^{\dagger}} / \varepsilon L_{r^{\dagger}}$.

Lemma 3.3.2. We have the following bijection

$$
\begin{aligned}
& \left\{\text { lattices } L^{\prime} \text { in } V \mid L_{-1} \subseteq\left(L^{\prime}\right)^{\#} \subseteq L^{\prime} \stackrel{1}{\subset} L_{0}\right\} \stackrel{\sim}{\longrightarrow} \\
& \left\{k \text {-subspaces } W \text { of } L_{0} / \varepsilon L_{r^{\dagger}} \mid L_{-1} / \varepsilon L_{r^{\dagger}} \subseteq W \stackrel{1}{\subset} L_{0} / \varepsilon L_{r^{\dagger}}, W^{\perp} \subseteq W\right\},
\end{aligned}
$$

which sends $L^{\prime}$ to $L^{\prime} / \varepsilon L_{r^{\dagger}}$. (Here and below $\stackrel{1}{\subset}$ denotes subspaces of codimension 1.$)$

Therefore, the computation at $L_{0}$ is exactly the same as the computation at $L_{r}$. In particular, we have the following lemma which we shall use freely.

Lemma 3.3.3. (1) Suppose that $L$ is a lattice such that $L_{-1} \subset L \subset L_{0}$ and $\operatorname{dim}_{k} L_{0} / L=$ 1 , then the lattice $L$ is symplectic and $L^{\#} \subset L$.

(2) If the pair $\left(L_{-1}, L_{0}\right)$ is replaced by $\left(L_{r}, L_{r+1}\right)$ such that $\operatorname{dim}_{k} L / L_{r}=1$, then $L$ is symplectic and $L \subset L^{\#}$. 


\section{CHAPTER 4}

\section{Multiplication formulas for Chevalley generators}

In this chapter, we study the convolution algebra $\mathbf{S}_{n, d}^{\mathfrak{c}}$ of pairs of $n$-step flags of affine type $C$. We present multiplication formulas in $\mathbf{S}_{n, d}^{\mathfrak{c}}$ with (the divided powers of) Chevalley generators. We then specify some general scenarios when these multiplication formulas produce a leading term with coefficient 1.

\subsection{Some Dimension COMPUTATION}

Fix $L \in \mathcal{X}_{n, d}^{\mathfrak{c}}$. For $A \in{ }^{\mathfrak{c}} \Xi_{n, d}$ (which was defined in (3.2.4)), we define

$$
X_{A}^{L}=\left\{L^{\prime} \in \mathcal{X}_{n, d}^{\mathfrak{c}} \mid\left(L, L^{\prime}\right) \in \mathcal{O}_{A}\right\}
$$

This is an orbit of the stabilizer subgroup $\operatorname{Stab}_{\mathrm{Sp}(V)}(L)$ of $\operatorname{Sp}(V)$, and one can associate to it a structure of quasi-projective algebraic variety. We are interested in computing its dimension $d_{A}^{\mathfrak{c}}$ (in order to define the standard basis element [A] later on). We have the following affine type $C$ analogue of [Lu99, Lemma 4.3].

Lemma 4.1.1. Fix $L \in \mathcal{X}_{n, d}^{\mathfrak{c}}$. For $A \in{ }^{\mathfrak{c}} \Xi_{n, d}$, the dimension of $X_{A}^{L}$ is given by

$$
d_{A}^{\mathfrak{c}}=\frac{1}{2}\left(\sum_{\substack{i \geqslant k, j<l \\ i \in[1, n]}} a_{i j} a_{k l}+\sum_{i \geqslant 0>j} a_{i j}+\sum_{i \geqslant r+1>j} a_{i j}\right) .
$$

Proof. The proof is similar to that of in [Lu99, Lemma 4.3]. Indeed, we can fix a decomposition $V=\oplus_{i, j \in \mathbb{Z}} V_{i j}$ as $k$-vector spaces such that $\operatorname{dim}_{k} V_{i j}=a_{i j}$. We can further assume that $V_{i j}$ admits a $k$-basis $\left\{e_{i j}^{m} \mid 1 \leqslant m \leqslant a_{i j}\right\}$ satisfying

$$
\varepsilon e_{i j}^{m}=e_{i-n, j-n}^{m}, \quad \forall i, j \in \mathbb{Z}, m \in\left[1, a_{i j}\right] .
$$

We define a symplectic $F$-form on $V$ by, for any $i, k \in[1, n], j, l \in \mathbb{Z}$,

$$
\left(e_{i j}^{m}, e_{k l}^{m^{\prime}}\right)= \begin{cases}\delta_{m, m^{\prime}} \varepsilon^{-2}, & \text { if } i+k=n, j+l=n, i<r+1, \\ -\delta_{m, m^{\prime}} \varepsilon^{-2}, & \text { if } i+k=n, j+l=n, i>r+1, \\ \delta_{m, m^{\prime}} \varepsilon^{-2}, & \text { if } i+k=n, j+l=n, i=r+1, j<r+1, \\ -\delta_{m, m^{\prime}} \varepsilon^{-2}, & \text { if } i+k=n, j+l=n, i=r+1, j>r+1, \\ \delta_{m, a_{r+1, r+1}+1-m^{\prime}} \varepsilon^{-2}, & \text { if }(i, j)=(k, l)=(r+1, r+1), m \leqslant a_{r+1, r+1} / 2, \\ -\delta_{m, a_{r+1, r+1}+1-m^{\prime}} \varepsilon^{-2}, & \text { if }(i, j)=(k, l)=(r+1, r+1), m \geqslant a_{r+1, r+1} / 2+1, \\ \varepsilon\left(e_{i j}^{m}, e_{k-n, l-n}^{m^{\prime}}\right) . & \end{cases}
$$

Now set $L=\left(L_{i}\right)_{i \in \mathbb{Z}}$ and $L^{\prime}=\left(L_{j}^{\prime}\right)_{j \in \mathbb{Z}}$, where

$$
L_{i}=\bigoplus_{k, l \in \mathbb{Z}: k \leqslant i} V_{k l}, \quad L_{j}^{\prime}=\bigoplus_{k, l \in \mathbb{Z}: l \leqslant j} V_{k l}, \quad \forall i, j \in \mathbb{Z}
$$


We see that $\left(L, L^{\prime}\right) \in \mathcal{O}_{A}$. Let

$$
X=\{x \in \mathfrak{s p}(V) \mid x(L) \subseteq L\}, \quad X^{\prime}=\left\{x \in \mathfrak{s p}(V) \mid x(L) \subseteq L, x\left(L^{\prime}\right) \subseteq L^{\prime}\right\}
$$

We have

$$
\operatorname{dim} X_{A}^{L}=\operatorname{dim}_{k} X / X^{\prime} .
$$

Now $x=\left(x_{(i, j),(k, l)}: V_{i j} \rightarrow V_{k l}\right) \in \mathfrak{s p}(V)$ if and only if the following conditions are satisfied:

$$
\begin{aligned}
x_{(n+i, n+j),(n+k, n+l)}(u) & =\varepsilon^{-1} x_{(i, j),(k, l)}(\varepsilon u), \quad \forall u \in V_{n-i, n-j}, \\
\left(x(u), u^{\prime}\right)+\left(u, x\left(u^{\prime}\right)\right) & =0, \quad \forall u, u^{\prime} \in V .
\end{aligned}
$$

The second condition in (4.1.2) is equivalent to

$$
{ }^{t} x_{(i, j),(k, l)} M+M x_{(s n-k, s n-l),(s n-i, s n-j)}=0, \quad \forall i, j, k, l, s \in \mathbb{Z},
$$

where $M$ is a certain matrix associated to the symplectic form $(\cdot, \cdot)$.

In particular, if $i+k \neq s n$ or $j+l \neq s n, \forall s \in \mathbb{Z}$, then the linear map $x_{(i, j),(k, l)}$ completely determines $x_{(s n-k, s n-l),(s n-i, s n-j)}$ for all $s \in \mathbb{Z}$. So the contribution for these linear maps in $\operatorname{dim} X / X^{\prime}$ is

$$
\frac{1}{2} \sum_{\substack{i \geqslant k, j<l, i \in[0, n-1] \\ i+k \neq s n \text { or } j+l \neq s n}} a_{i j} a_{k l} .
$$

If we have $i+k=s n$ or $j+l=s n$ for some $s \in \mathbb{Z}$, then the equation (4.1.3) becomes ${ }^{t} x_{(i, j),(k, l)} M+M x_{(i, j),(k, l)}=0$. By (4.1.2), the collection of linear maps $x_{(i, j),(k, l)}$ such that $i+k=s n$ and $j+l=s n$ for some $s \in \mathbb{Z}$ determines the collection of linear maps $x_{(i, j),(k, l)}$ such that $i+k=(s+2) n$ and $j+l=(s+2) n$. So they are determined by the following two subsets:

$$
\left\{x_{(i j),(k l)} \mid i+k=0, j+l=0\right\}, \quad\left\{x_{(i j),(k l)} \mid i+k=n, j+l=n\right\} .
$$

So the contribution of these kind of linear maps to $\operatorname{dim} X / X^{\prime}$ is

$$
\frac{1}{2} \sum_{\substack{i \geqslant k, j<l, i \in[0, n-1] \\ i+k=s n, j+l=s n}} a_{i j} a_{k l}+\frac{1}{2} \sum_{i \geqslant 0>j} a_{i j}+\frac{1}{2} \sum_{i \geqslant r+1>j} a_{i j} .
$$

The lemma follows by summing up (4.1.4) and (4.1.5).

\subsection{Standard AND CANONiCAL BASEs of Schur Algebras}

It turns out a "type $B$ " parametrization in place of the "type $C$ " parametrization via ${ }^{c} \Xi_{n, d}$ is more natural, for the $\operatorname{Sp}(V)$-orbits in $\mathcal{X}_{n, d}^{\mathfrak{c}} \times \mathcal{X}_{n, d}^{\mathfrak{c}}$ and then for bases of the Schur algebras later on. (Such a phenomenon already occurred in the finite type; cf. [BKLW14, [FL15].) We introduce the "type $B$ " parametrization set

$$
\Xi_{n, d}=\left\{A+E^{00}+E^{r+1, r+1} \mid A \in{ }^{\mathfrak{c}} \Xi_{n, d}\right\} .
$$

That is, $\Xi_{n, d}$ is the set of matrices $A \in \operatorname{Mat}_{\mathbb{Z} \times \mathbb{Z}}(\mathbb{N})$ subject to the following conditions:

$$
\begin{aligned}
& a_{i j}=a_{-i,-j}=a_{i-n, j-n}(\forall i, j \in \mathbb{Z}), \quad a_{00}, a_{r+1, r+1} \in 2 \mathbb{Z}+1, \\
& \sum_{i=i_{0}+1}^{i_{0}+n} \sum_{j \in \mathbb{Z}} a_{i j}=2 d+2, \quad \text { for one (or each) } i_{0} \in \mathbb{Z} .
\end{aligned}
$$


By definition we have a bijection

$$
{ }^{\mathfrak{c}} \Xi_{n, d} \longleftrightarrow \Xi_{n, d}, \quad A \mapsto A+E^{00}+E^{r+1, r+1}
$$

From now on, we shall switch to the indexing set $\Xi_{n, d}$ for the rest of the paper. Hence by abuse of notations, an $\operatorname{Sp}_{F}(2 d)$-orbit on $\mathcal{X}_{n, d}^{\mathfrak{c}} \times \mathcal{X}_{n, d}^{\mathfrak{c}}$ is denoted by $\mathcal{O}_{A}$ and the set in (4.1.1) is denoted by $X_{A}^{L}$, now for $A \in \Xi_{n, d}$.

Lemma 4.2.1. The dimension of $X_{A}^{L}$ for $A \in \Xi_{n, d}$ is given by

$$
d_{A}=\frac{1}{2}\left(\sum_{\substack{i \geqslant k, j<l \\ i \in[0, n-1]}} a_{i j} a_{k l}-\sum_{i \geqslant 0>j} a_{i j}-\sum_{i \geqslant r+1>j} a_{i j}\right) .
$$

Proof. Let $A=\left(a_{i j}\right) \in{ }^{\mathfrak{c}} \Xi_{n, d}$. We shall denote $A^{\prime}=\left(a_{i j}^{\prime}\right) \in \Xi_{n, d}$ corresponding to $A$ via the bijection (4.2.3). Thus we have

$$
a_{i j}^{\prime}=a_{i j}+\delta_{i j} \sum_{k \in \mathbb{Z}} \delta_{0, i+k n}+\delta_{i j} \sum_{k \in \mathbb{Z}} \delta_{r+1, i+k n} .
$$

It follows from Lemma 4.1.1 that

$$
\begin{aligned}
d_{A^{\prime}}=d_{A}^{\mathfrak{c}}= & \frac{1}{2}\left(\sum_{\substack{i \geqslant k, j<l \\
i \in[1, n]}} a_{i j} a_{k l}+\sum_{i \geqslant 0>j} a_{i j}+\sum_{i \geqslant r+1>j} a_{i j}\right) \\
= & \frac{1}{2}\left(\sum_{\substack{i \geqslant k, j<l \\
i \in[1, n]}} a_{i j}^{\prime} a_{k l}^{\prime}-\sum_{l>0 \geqslant k} a_{k l}^{\prime}-\sum_{l>r+1 \geqslant k} a_{k l}^{\prime}-\sum_{\substack{i, j, k: i \geqslant k n>j \\
i \in[1, n]}} a_{i j}^{\prime}\right. \\
& \left.-\sum_{\substack{i, j, k: i \geqslant r+1+k n>j \\
i \in[1, n]}} a_{i j}^{\prime}+\sum_{i \geqslant 0>j} a_{i j}^{\prime}+\sum_{i \geqslant r+1>j} a_{i j}^{\prime}\right) \\
= & \frac{1}{2}\left(\sum_{\substack{i \geqslant k, j<l \\
i \in[1, n]}} a_{i j}^{\prime} a_{k l}^{\prime}-\sum_{i \geqslant 0>j} a_{i j}^{\prime}-\sum_{i \geqslant r+1>j} a_{i j}^{\prime}\right) .
\end{aligned}
$$

The lemma is proved.

We also introduce

$$
\begin{aligned}
\Sigma_{d}=\left\{A \in \operatorname{Mat}_{\mathbb{Z} \times \mathbb{Z}}(\{0,1\}) \mid\right. & a_{-i,-j}=a_{i j}=a_{i+2 d+2, j+2 d+2}(\forall i, j \in \mathbb{Z}), \\
& \exists \text { exactly one nonzero entry per row/column }\} .
\end{aligned}
$$

Note the description of $\Sigma_{d}$ is much cleaner than ${ }^{\mathrm{c}} \Sigma_{d}$, cf. (3.1.6). Nevertheless, the bijection (4.2.3) induces a natural bijection

$$
{ }^{\mathfrak{c}} \Sigma_{d} \longleftrightarrow \Sigma_{d}, \quad A \mapsto A+E^{00}+E^{r+1, r+1} .
$$

The bijection $\varphi: \operatorname{Sp}_{F}(2 d) \backslash \mathcal{Y}^{\mathfrak{c}} \times \mathcal{Y}^{\mathfrak{c}} \longrightarrow{ }^{\mathfrak{c}} \Sigma_{d}$ in Proposition 3.1.3 can be reformulated using $\Sigma_{d}$ in place of ${ }^{\mathfrak{c}} \Sigma_{d}$. 
Recall the Schur algebra of affine type $A, \mathbf{S}_{n, d ; \mathcal{A}}$, was defined in Section 2.2 . The $\mathcal{A}$-algebra $\mathbf{S}_{n, d ; \mathcal{A}}^{\mathfrak{c}}$ is defined in the same way, now as the (generic) convolution algebra $\mathcal{A}_{\mathrm{Sp}_{F}(2 d)}\left(\mathcal{X}_{n, d}^{\mathfrak{c}} \times\right.$ $\left.\mathcal{X}_{n, d}^{\mathfrak{c}}\right)$ attached to the variety $\mathcal{X}_{n, d}^{\mathfrak{c}}$ introduced in the previous chapter. We then set

$$
\mathbf{S}_{n, d}^{\mathfrak{c}}=\mathbb{Q}(v) \otimes_{\mathcal{A}} \mathbf{S}_{n, d ; \mathcal{A}}^{\mathfrak{c}} .
$$

The algebras $\mathbf{S}_{n, d ; \mathcal{A}}^{\mathfrak{c}}$ and $\mathbf{S}_{n, d}^{\mathfrak{c}}$ are called the Schur algebras (of affine type $C$ ). Denote by $e_{A}$ the characteristic function of the orbit $\mathcal{O}_{A}$, for $A \in \Xi_{n, d}$. Then $\left\{e_{A} \mid A \in \Xi_{n, d}\right\}$ forms a basis for $\mathbf{S}_{n, d ; \mathcal{A}}^{\mathfrak{c}}$ and $\mathbf{S}_{n, d}^{\mathfrak{c}}$. Set

$$
[A]=v^{-d_{A}} e_{A}, \quad \text { for } A \in \Xi_{n, d}
$$

Remark 4.2.2. We have

$$
d_{A}-d_{t_{A}}=\frac{1}{4}\left(\sum_{1 \leqslant i \leqslant n}\left(\operatorname{ro}(A)_{i}^{2}-\operatorname{co}(A)_{i}^{2}\right)-\left(\operatorname{ro}(A)_{0}-\operatorname{co}(A)_{0}\right)-\left(\operatorname{ro}(A)_{r+1}-\operatorname{co}(A)_{r+1}\right)\right) \text {. }
$$

Hence the assignment $[A] \mapsto\left[{ }^{t} A\right]$ defines an anti-involution $\Psi: \mathbf{S}_{n, d}^{\mathfrak{c}} \rightarrow \mathbf{S}_{n, d}^{\mathfrak{c}}$. Note from [Lu99, $1.6(\mathrm{a})]$ that

$$
\sum_{i \geqslant-(r+1)>j} a_{i j}=\operatorname{dim}_{k} \frac{L_{-r-2}^{\prime}}{L_{-r-2} \cap L_{-r-2}^{\prime}}, \quad \sum_{i \geqslant r+1>j} a_{i j}=\operatorname{dim}_{k} \frac{L_{r}^{\prime}}{L_{r} \cap L_{r}^{\prime}},
$$

for any $\left(L, L^{\prime}\right) \in \mathcal{O}_{A}$.

Recall the partial orders $\leqslant_{\text {alg }}$ and $\leqslant$ on $\Theta_{n, d}$ from (2.2.8) and (2.2.9). These two partial orders restrict to similar ones on $\Xi_{n, d}$, still denoted by the same notations. Since any matrix $A$ in $\Xi_{n, d}$ satisfies that $a_{i j}=a_{-i,-j}$ for all $i, j \in \mathbb{Z}$. The two conditions in (2.2.8) are equivalent to each other. Hence, the partial order $\leqslant_{\text {alg }}$ on $\Xi_{n, d}$ can be simplified as follows. Given any $A=\left(a_{i j}\right), A^{\prime}=\left(a_{i j}^{\prime}\right) \in \Xi_{n, d}$, one has

$$
A \leqslant \text { alg } A^{\prime} \Longleftrightarrow \sum_{k \leqslant i, l \geqslant j} a_{k l} \leqslant \sum_{k \leqslant i, l \geqslant j} a_{k l}^{\prime}, \quad \forall i<j .
$$

Since the Bruhat order of affine type $C$ is compatible with the Bruhat order of affine type $A$, we see that the partial order " $\leqslant$ " is compatible with (though possibly weaker than) the Bruhat order of affine type $C$.

Assume for now that the ground field is $\overline{\mathbb{F}}_{q}$. Let $I C_{A}$ be the intersection cohomology complex of the closure $\overline{X_{A}^{L}}$ of $X_{A}^{L}$, taken in certain ambient algebraic variety over $\overline{\mathbb{F}}_{q}$, such that the restriction of the stratum $I C_{A}$ to $X_{A}^{L}$ is the constant sheaf on $X_{A}^{L}$. We refer to BBD82 for the precise definition of intersection complexes. The restriction of the $i$-th cohomology sheaf $\mathscr{H}_{X_{B}^{L}}^{i}\left(I C_{A}\right)$ of $I C_{A}$ to $X_{B}^{L}$ for $B \leqslant A$ is a trivial local system, whose rank is denoted by $n_{B, A, i}$. We set

$$
\{A\}_{d}=\sum_{B \leqslant A} P_{B, A}[B], \quad \text { where } \quad P_{B, A}=\sum_{i \in \mathbb{Z}} n_{B, A, i} v^{i-d_{A}+d_{B}} .
$$

The polynomials $P_{B, A}$ satisfy

$$
P_{A, A}=1 \quad \text { and } \quad P_{B, A} \in v^{-1} \mathbb{Z}\left[v^{-1}\right] \text { for any } B<A .
$$


Recall $\left\{[A] \mid A \in \Xi_{n, d}\right\}$ forms an $\mathbb{Q}(v)$-basis of $\mathbf{S}_{n, d}^{\mathfrak{c}}$. In light of [BBD82, Lu97], we have the following.

Proposition 4.2.3. The set $\left\{\{A\}_{d} \mid A \in \Xi_{n, d}\right\}$ forms an $\mathcal{A}$-basis of $\mathbf{S}_{n, d ; \mathcal{A}}^{\mathfrak{c}}$ and a $\mathbb{Q}(v)$-basis of $\mathbf{S}_{n, d}^{\mathfrak{c}}$ (called the canonical basis). Moreover, the structure constants of $\mathbf{S}_{n, d}^{\mathfrak{c}}$ with respect to the canonical basis are in $\mathbb{N}\left[v, v^{-1}\right]$.

\subsection{Some multiplication formulas}

Recalling $E^{i j}$ from (2.2.4), we set

$$
E_{\theta}^{i j}=E^{i j}+E^{-i,-j}
$$

Note that we have

$$
E_{\theta}^{00}=2 E^{00}, \quad E_{\theta}^{r+1, r+1}=2 E^{r+1, r+1} .
$$

We have the following affine analogue of [BKLW14, Lemma 3.2], whose proof also explains why the formula therein is the same as those in [BLM90].

Lemma 4.3.1. Assume that $i \in \mathbb{Z}$ and $A, B, C \in \Xi_{n, d}$.

(1) If $\operatorname{ro}(A)=\operatorname{co}(B)$ and $B-E_{\theta}^{i, i+1}$ is diagonal, then we have

$$
e_{B} * e_{A}=\sum_{\substack{p \in \mathbb{Z} \\ a_{i+1, p} \geqslant\left(E_{\theta}^{i+1, p}\right)_{i+1, p}}} v^{2 \sum_{j>p} a_{i j}} \frac{v^{2\left(1+a_{i p}\right)}-1}{v^{2}-1} e_{A+E_{\theta}^{i p}-E_{\theta}^{i+1, p} .}
$$

(2) If $\operatorname{ro}(A)=\operatorname{co}(C)$ and $C-E_{\theta}^{i+1, i}$ is diagonal, then we have

$$
e_{C} * e_{A}=\sum_{\substack{p \in \mathbb{Z} \\ a_{i, p} \geqslant\left(E_{\theta}^{i, p}\right)_{i, p}}} v^{2 \sum_{j<p} a_{i+1, j}} \frac{v^{2\left(1+a_{i+1, p}\right)}-1}{v^{2}-1} e_{A-E_{\theta}^{i p}+E_{\theta}^{i+1, p} .}
$$

Proof. The proof is essentially the same as that of [Lu99, Proposition 3.5]. Obtaining the structure constant in the first formula is reduced to computing the orders of the following two sets:

$$
\begin{gathered}
\left\{U \text { symplectic lattice } \mid L_{i-1}+\left(L_{i} \cap L_{p-1}^{\prime}\right) \subseteq U \subseteq L_{i} \operatorname{dim}_{k} L_{i} / U=1\right\} \\
\left\{U \text { symplectic lattice } \mid L_{i-1}+\left(L_{i} \cap L_{p}^{\prime}\right) \subseteq U \subseteq L_{i}, \operatorname{dim}_{k} L_{i} / U=1\right\}
\end{gathered}
$$

Since the lattices $U$ such that $L_{i-1} \subseteq U \stackrel{1}{\subset} L_{i}$ are automatically symplectic (and $U^{\#} \subseteq U$ ) by Lemma 3.3.3, the computations in loc. cit. still work and we have the first formula.

For the second formula, it is reduced to computing the difference of the orders of the following two sets:

$$
\begin{gathered}
\left\{U \text { symplectic lattice } \mid L_{i} \subseteq U \subseteq L_{i}+\left(L_{i+1} \cap L_{p}^{\prime}\right), \operatorname{dim}_{k} U / L_{i}=1\right\}, \\
\left\{U \text { symplectic lattice } \mid L_{i} \subseteq U \subseteq L_{i}+\left(L_{i+1} \cap L_{p-1}^{\prime}\right), \operatorname{dim}_{k} U / L_{i}=1\right\} .
\end{gathered}
$$

And again in this case, the lattices involved are automatically symplectic and thus the computations in loc. cit. work here again. The second formula is obtained.

We now generalize Lemma 4.3.1 to a multiplication formula by "divided powers" of Chevalley generators. 
Lemma 4.3.2. Assume that $A, B, C \in \Xi_{n, d}$ and $R \in \mathbb{N}$.

(1) If $\operatorname{ro}(A)=\operatorname{co}(B)$ and $B-R E_{\theta}^{i, i+1}$ is diagonal for some $i \in[1, r]$, then we have

$$
e_{B} * e_{A}=\sum_{t} v^{2 \sum_{j>u} a_{i j} t_{u}} \prod_{u \in \mathbb{Z}}\left[\begin{array}{c}
a_{i u}+t_{u} \\
t_{u}
\end{array}\right] e_{A+\sum_{u \in \mathbb{Z}} t_{u}\left(E_{\theta}^{i u}-E_{\theta}^{i+1, u}\right)},
$$

where the sum is over all sequences $t=\left(t_{u} \mid u \in \mathbb{Z}\right)$ such that $t_{u} \in \mathbb{N}$ and $\sum_{u \in \mathbb{Z}} t_{u}=R$.

(2) If $\operatorname{ro}(A)=\operatorname{co}(B)$ and $B-R E_{\theta}^{0,1}$ is diagonal, then we have

$$
\begin{aligned}
e_{B} * e_{A}=\sum_{t} v^{2 \sum_{j>u} a_{0 j} t_{u}+2 \sum_{j<u<-j} t_{j} t_{u}+\sum_{u<0} t_{u}\left(t_{u}-1\right)} \\
\cdot \prod_{u>0}\left[\begin{array}{c}
a_{0 u}+t_{u}+t_{-u} \\
t_{u}
\end{array}\right] \prod_{u<0}\left[\begin{array}{c}
a_{0 u}+t_{u} \\
t_{u}
\end{array}\right] \prod_{i=0}^{t_{0}-1} \frac{\left[a_{00}+1+2 i\right]}{[i+1]} e_{A+\sum_{u \in \mathbb{Z}} t_{u}\left(E_{\theta}^{0 u}-E_{\theta}^{1 u}\right)} .
\end{aligned}
$$

(3) If $\operatorname{ro}(A)=\operatorname{co}(C)$ and $C-R E_{\theta}^{i+1, i}$ is diagonal for some $i \in[0, r-1]$, then we have

$$
e_{C} * e_{A}=\sum_{t} v^{2 \sum_{j<u} a_{i+1, j} t_{u}} \prod_{u \in \mathbb{Z}}\left[\begin{array}{c}
a_{i+1, u}+t_{u} \\
t_{u}
\end{array}\right] e_{A-\sum_{u \in \mathbb{Z}} t_{u}\left(E_{\theta}^{i u}-E_{\theta}^{i+1, u}\right)} .
$$

(4) If $\operatorname{ro}(A)=\operatorname{co}(C)$ and $C-R E_{\theta}^{r+1, r}$ is diagonal, then we have

$$
\begin{aligned}
e_{C} * e_{A} & =\sum_{t} v^{2 \sum_{j<u} a_{r+1, j} t_{u}+2 \sum_{n-j<u<j} t_{u} t_{j}+\sum_{u>r+1} t_{u}\left(t_{u}-1\right)} \prod_{u<r+1}\left[\begin{array}{c}
a_{r+1, u}+t_{u} \\
t_{u}
\end{array}\right] \\
& \cdot \prod_{u>r+1}\left[\begin{array}{c}
a_{r+1, u}+t_{u}+t_{n-u} \\
t_{u}
\end{array}\right] \prod_{i=0}^{t_{r+1}-1} \frac{\left[a_{r+1, r+1}+1+2 i\right]}{[i+1]} e_{A-\sum_{u \in \mathbb{Z}} t_{u}\left(E_{\theta}^{r u}-E_{\theta}^{r+1, u}\right)},
\end{aligned}
$$

where the sum is taken over $t=\left(t_{u} \mid u \in \mathbb{Z}\right)$ such that $t_{u} \in \mathbb{N}$ and $\sum_{u \in \mathbb{Z}} t_{u}=R$.

Proof. The proofs of (1), (3) and (4) are essentially the same as that of [BKLW14, Proposition 3.3], while the proof of (2) is similar to the proof of (4). Let us give a proof of (2) and skip (1), (3) and (4). We shall prove by induction on $R$. When $R=1$, we have (2) by Lemma 4.3.1. Write $B_{R}$ for $B$ in order to keep track of the $R$, and $A_{t}$ for the matrix $A+\sum_{u \in \mathbb{Z}} t_{u}\left(E_{\theta}^{0 u}-E_{\theta}^{1 u}\right)$ associated with $t$. Let $G_{A, t}$ denote the coefficient of $e_{A_{t}}$ in (2). By Lemma 4.3.1, we have $e_{B_{1}} * e_{B_{R}}=[R+1] e_{B_{R+1}}$. So

$$
e_{B_{R+1}} * e_{A}=\frac{1}{[R+1]} \sum_{t, \underline{p}} G_{A, t} G_{A_{t}, \underline{p}} e_{A_{t+\underline{p}}}
$$

where $p \in \mathbb{N}^{\mathbb{Z}}$ is the sequence whose nonzero entry is 1 at the position $p$.

It suffices to show that

$$
\frac{1}{[R+1]} \sum_{t, \underline{p}: t+\underline{p}=s} G_{A, t} G_{A_{t}, \underline{p}}=G_{A, s}
$$

for any sequence $s \in \mathbb{N}^{\mathbb{Z}}$ such that $\sum_{u \in \mathbb{Z}} s_{u}=R+1$. By Lemma 4.3.1, the coefficient $G_{A_{t}, \underline{p}}$ is equal to $v^{2 \sum_{j>p} a_{0 j}+2 \sum_{j>p}\left(t_{j}+t_{-j}\right)}\left[a_{0 p}+t_{p}+t_{-p}+1\right]$. The $v$-power terms of $G_{A, t}$ and $G_{A_{t}, \underline{p}}$ together yield the $v$-power term of $G_{A, s}$ multiplying with $v^{2 \sum_{j>p} t_{j}}$. The $v$-binomial 
coefficients of $G_{A, t}$ and $G_{A_{t}, \underline{p}}$ yield the $v$-binomial coefficient of $G_{A, s}$ multiplying with $\left[s_{p}\right]$. So we have

$$
\frac{1}{[R+1]} \sum_{t+\underline{p}=s} G_{A, t} G_{A_{t}, \underline{p}}=G_{A, s} \frac{1}{[R+1]} \sum_{p \in \mathbb{Z}} v^{2 \sum_{j>p} t_{j}}\left[r_{p}\right]=G_{A, s} .
$$

By induction, we have proved (2).

Lemma 4.3 .2 can be rewritten in terms of the standard basis $[A]$ as follows. Recall that we have a bar involution ${ }^{-}: \mathbb{Q}(v) \rightarrow \mathbb{Q}(v)$ defined by $\bar{v}=v^{-1}$.

Proposition 4.3.3. Assume that $A, B, C \in \Xi_{n, d}$ and $R \in \mathbb{N}$.

(1) If $\operatorname{ro}(A)=\operatorname{co}(B)$ and $B-R E_{\theta}^{i, i+1}$ is diagonal for some $i \in[1, r]$, then we have

$$
\begin{gathered}
{[B] *[A]=\sum_{t} v^{\beta_{t}} \prod_{u \in \mathbb{Z}} \overline{\left[\begin{array}{c}
a_{i u}+t_{u} \\
t_{u}
\end{array}\right]}\left[A+\sum_{u \in \mathbb{Z}} t_{u}\left(E_{\theta}^{i u}-E_{\theta}^{i+1, u}\right)\right],} \\
\beta_{t}=\sum_{j \geqslant u} a_{i j} t_{u}-\sum_{j>u} a_{i+1, j} t_{u}+\sum_{j<u} t_{j} t_{u}+\frac{1}{2} \delta_{i, r}\left(\sum_{j+u<n} t_{j} t_{u}+\sum_{j<r+1} t_{j}\right),
\end{gathered}
$$

where the sum is over all sequences $t=\left(t_{u} \mid u \in \mathbb{Z}\right)$ such that $t_{u} \in \mathbb{N}$ and $\sum_{u \in \mathbb{Z}} t_{u}=R$.

(2) If $\operatorname{ro}(A)=\operatorname{co}(B)$ and $B-R E_{\theta}^{0,1}$ is diagonal, then we have

$[B] *[A]$

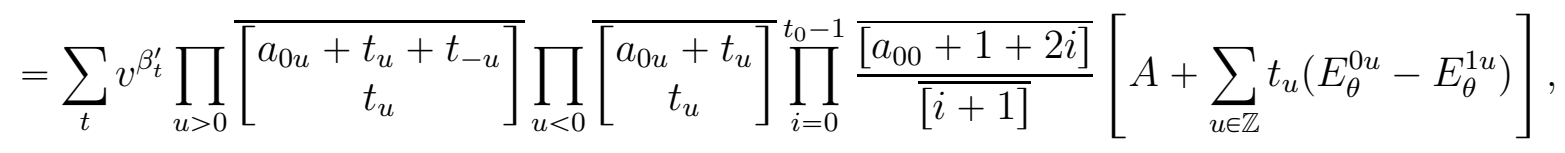

where the sum is over all sequences $t=\left(t_{u} \mid u \in \mathbb{Z}\right)$ such that $t_{u} \in \mathbb{N}$ and $\sum_{u \in \mathbb{Z}} t_{u}=R$, and

$$
\beta_{t}^{\prime}=\sum_{j \geqslant u} a_{0 j} t_{u}-\sum_{j>u} a_{1 j} t_{u}+\sum_{j<u, j+u \leqslant 0} t_{j} t_{u}-\sum_{j>0} \frac{t_{j}^{2}-t_{j}}{2}+\frac{R^{2}-R}{2} .
$$

(3) If $\operatorname{ro}(A)=\operatorname{co}(C)$ and $C-R E_{\theta}^{i+1, i}$ is diagonal for some $i \in[0, r-1]$, then we have

$$
[C] *[A]=\sum_{t} v^{\gamma_{t}} \prod_{u \in \mathbb{Z}} \overline{\left[\begin{array}{c}
a_{i+1, u}+t_{u} \\
t_{u}
\end{array}\right]}\left[A-\sum_{u \in \mathbb{Z}} t_{u}\left(E_{\theta}^{i u}-E_{\theta}^{i+1, u}\right)\right],
$$

where the sum is over all sequences $t=\left(t_{u} \mid u \in \mathbb{Z}\right)$ such that $t_{u} \in \mathbb{N}$ and $\sum_{u \in \mathbb{Z}} t_{u}=R$, and

$$
\gamma_{t}=\sum_{j \leqslant u} a_{i+1, j} t_{u}-\sum_{j<u} a_{i j} t_{u}+\sum_{j<u} t_{j} t_{u}+\frac{1}{2} \delta_{i, 0}\left(\sum_{j+u>0} t_{j} t_{u}+\sum_{j>0} t_{j}\right) .
$$

(4) If $\operatorname{ro}(A)=\operatorname{co}(C)$ and $C-R E_{\theta}^{r+1, r}$ is diagonal, then we have

$$
\begin{array}{r}
{[C] *[A]=\sum_{t} v^{\gamma_{t}^{\prime}} \prod_{u<r+1} \overline{\left[\begin{array}{c}
a_{r+1, u}+t_{u} \\
t_{u}
\end{array}\right]} \prod_{u>r+1} \overline{\left[\begin{array}{c}
a_{r+1, u}+t_{u}+t_{n-u} \\
t_{u}
\end{array}\right]}} \\
\prod_{i=0}^{t_{r+1}-1} \frac{\overline{\left[a_{r+1, r+1}+1+2 i\right]}}{\overline{[i+1]}}\left[A-\sum_{u \in \mathbb{Z}} t_{u}\left(E_{\theta}^{r u}-E_{\theta}^{r+1, u}\right)\right],
\end{array}
$$


where

$$
\gamma_{t}^{\prime}=\sum_{j \leqslant u} a_{r+1, j} t_{u}-\sum_{j<u} a_{r j} t_{u}+\sum_{j<u, j+u \geqslant n} t_{j} t_{u}-\sum_{j<r+1} \frac{t_{j}^{2}-t_{j}}{2}+\frac{R^{2}-R}{2},
$$

and $t=\left(t_{u} \mid u \in \mathbb{Z}\right)$ such that $t_{u} \in \mathbb{N}$ and $\sum_{u \in \mathbb{Z}} t_{u}=R$.

Proof. Let us prove (1). By definition, we have $d_{B}=R b_{i i}=\sum_{j, u} a_{i j} t_{u}$. Let us denote $(k, l)$-th entry in $A+\sum_{u \in \mathbb{Z}} t_{u}\left(E_{\theta}^{i u}-E_{\theta}^{i+1, u}\right)$ by ${ }^{i} a_{k l}$. A lengthy calculation yields

$$
\sum{ }^{i} a_{i j}{ }^{i} a_{k l}-\sum a_{i j} a_{k l}=2 \sum_{j<u} a_{i j} t_{u}-2 \sum_{j>u} a_{i+1, j} t_{u}+2 \sum_{j<u} t_{j} t_{u}+\delta_{i, r} \sum_{j+u<n} t_{j} t_{u},
$$

where the sums on the left-hand side run over all $(i, j, k, l)$ such that $i \geqslant k, j<l$ and $i \in[0, n-1]$. We also have

$$
\begin{aligned}
\sum_{i \geqslant 0>j}{ }^{i} a_{i j} & =\sum_{i \geqslant 0>j} a_{i j}, \\
\sum_{i \geqslant r+1>j}{ }^{i} a_{i j} & =\sum_{i \geqslant r+1>j} a_{i j}-\delta_{i r} \sum_{j<r+1} t_{j} .
\end{aligned}
$$

Putting the above computations together, we have

$$
d_{A_{t}}-d_{A}=\sum_{j<u} a_{i j} t_{u}-\sum_{j>u} a_{i+1, j} t_{u}+\sum_{j<u} t_{j} t_{u}+\frac{1}{2} \delta_{i, r}\left(\sum_{j+u<n} t_{j} t_{u}+\sum_{j<r+1} t_{j}\right),
$$

where $A_{t}=A+\sum_{u \in \mathbb{Z}} t_{u}\left(E_{\theta}^{i u}-E_{\theta}^{i+1, u}\right)$. Now from Lemma 4.3.2(1), we have

$$
\beta_{t}=-d_{B}+d_{A_{t}}-d_{A}+2 \sum_{j>u} a_{i j} t_{u}+2 \sum_{u \in \mathbb{Z}} a_{i u} t_{u} .
$$

The above calculations give rise to the formula for $\beta_{t}$, and (1) follows.

We now prove (2). We set $A_{0, t}=A+\sum_{u \in \mathbb{Z}} t_{u}\left(E_{\theta}^{0 u}-E_{\theta}^{1 u}\right)$ and write its $(i, j)$-entry by ${ }^{0} a_{i j}$. We have

$$
\beta_{t}^{\prime}=-d_{B}-d_{A}+d_{A_{0, t}}+2 \sum_{j \geqslant u} a_{0 j} t_{u}+2 \sum_{j<u, j+u \leqslant 0} t_{j} t_{u}+\sum_{u \leqslant 0} t_{u}\left(t_{u}-1\right) .
$$

By definition, we have $d_{B}=\sum_{j, u} a_{0 j} t_{u}+\frac{R^{2}-R}{2}$. Moreover, we have

$$
d_{A_{0, t}}-d_{A}=\sum_{j<u} a_{0 j} t_{u}-\sum_{j>u} a_{1 j} t_{u}+\sum_{j<u} t_{j} t_{u}+\frac{1}{2}\left(\sum_{j+u>0} t_{j} t_{u}-\sum_{j>0} t_{j}\right) .
$$

Thus,

$$
\begin{aligned}
\beta_{t}^{\prime} & =\sum_{j \geqslant u} a_{0 j} t_{u}-\sum_{j>u} a_{1 j} t_{u}-\frac{R^{2}-R}{2}+\sum_{j<u} t_{j} t_{u} \\
& +2 \sum_{j<u, j+u \leqslant 0} t_{j} t_{u}+\sum_{u \leqslant 0} t_{u}\left(t_{u}-1\right)+\frac{1}{2}\left(\sum_{j+u>0} t_{j} t_{u}-\sum_{j>0} t_{j}\right) \\
& =\sum_{j \geqslant u} a_{0 j} t_{u}-\sum_{j>u} a_{1 j} t_{u}+\frac{R^{2}-R}{2}+\sum_{j<u, j+u \leqslant 0} t_{j} t_{u}-\frac{1}{2} \sum_{j>0} t_{j}^{2}-t_{j}
\end{aligned}
$$


So we have proved (2).

For (3), we have $d_{C}=\sum_{j, u} a_{i+1, j} t_{u}$, and

$$
d_{A-\sum_{u \in \mathbb{Z}} t_{u}\left(E_{\theta}^{i u}-E_{\theta}^{i+1, u}\right)}-d_{A}=\sum_{j>u} a_{i+1, j} t_{u}-\sum_{j<u} a_{i j} t_{u}+\sum_{j<u} t_{j} t_{u}+\frac{1}{2} \delta_{i, 0}\left(\sum_{j+u>0} t_{j} t_{u}+\sum_{j>0} t_{j}\right) .
$$

So we have the formula for $\gamma_{t}$ in (3).

For (4), we have $d_{C}=\sum_{j, u} a_{r+1, j} t_{u}+\frac{R^{2}-R}{2}$, and

$$
d_{A-\sum_{u \in \mathbb{Z}} t_{u}\left(E_{\theta}^{r u}-E_{\theta}^{r+1, u}\right)}-d_{A}=\sum_{j>u} a_{r+1, j} t_{u}-\sum_{j<u} a_{r j} t_{u}+\sum_{j<u} t_{j} t_{u}+\frac{1}{2}\left(\sum_{j+u<n} t_{j} t_{u}-\sum_{j<r+1} t_{j}\right) .
$$

So we have the formula for $\gamma_{t}^{\prime}$ in (4).

\subsection{THE LEADING TERM}

We have the following affine generalization of [BKLW14, Lemma 3.9].

Lemma 4.4.1. Let $A, B, C \in \Xi_{n, d}$. Let $R$ be a positive integer.

(1) Assume that $B-R E_{\theta}^{h, h+1}$ is diagonal for some $h \in[0, r]$ and $\operatorname{co}(B)=\operatorname{ro}(A)$. Assume further that the matrix A satisfies one of the following conditions:

$a_{0 j}=0, \forall j \geqslant k ; a_{1 k}=R, a_{1 j}=0, \forall j>k, \quad$ if $h=0, k \geqslant 0 ;$ or

$a_{h j}=0, \forall j \geqslant k ; a_{h+1, k}=R, a_{h+1, j}=0, \forall j>k, \quad$ if $h \in[1, r-1] ;$ or

$a_{r j}=0, \forall j \geqslant k ; a_{r+1, k}=R, a_{r+1, j}=0, \forall j>k, \quad$ if $h=r, k>r+1$; or

$a_{r j}=0, \forall j \geqslant r+1 ; a_{r+1, r+1} \geqslant 2 R, a_{r+1, j}=0, \forall j>r+1, \quad$ if $h=r, k=r+1$.

Then we have $[B] *[A]=\left[A+R\left(E_{\theta}^{h, k}-E_{\theta}^{h+1, k}\right)\right]+$ lower terms.

(2) Assume that $C-R E_{\theta}^{h+1, h}$ is diagonal for some $h \in[0, r]$ and $\operatorname{co}(C)=\operatorname{ro}(A)$. Assume further that $A$ satisfies one of the following conditions:

$$
\begin{aligned}
& a_{1 j}=0, \quad \forall j \leqslant k ; a_{0 k}=R, a_{0 j}=0, \quad \forall j<k, \quad \text { if } h=0, k<0 ; \text { or } \\
& a_{1 j}=0, \quad \forall j \leqslant k ; a_{0 k} \geqslant 2 R, a_{0 j}=0, \quad \forall j<k, \quad \text { if } h=0, k=0 ; \text { or } \\
& a_{h j}=0, \quad \forall j<k ; a_{h k}=R ; a_{h+1, j}=0, \quad \forall j \leqslant k, \quad \text { if } h \in[1, r-1] ; \text { or } \\
& a_{r j}=0, \quad \forall j<k ; a_{r k}=R ; a_{r+1, j}=0, \quad \forall j \leqslant k, \quad \text { if } h=r, k \leqslant r .
\end{aligned}
$$

Then we have $[C] *[A]=\left[A-R\left(E_{\theta}^{h, k}-E_{\theta}^{h+1, k}\right)\right]+$ lower terms.

Proof. We prove (1). Set

$$
M=A+R\left(E_{\theta}^{h, k}-E_{\theta}^{h+1, k}\right), \quad M^{\prime}=A+\sum_{u \in \mathbb{Z}} t_{u}\left(E_{\theta}^{h u}-E_{\theta}^{h+1, u}\right),
$$

with $\sum_{u \in \mathbb{Z}} t_{u}=R$. By an argument similar to the proof of [BLM90, Lemma 3.8], it is enough to show that $M^{\prime} \leqslant$ alg $M$. Assume that $h \in[1, r-1]$. By definition, the $(r, s)$-th entry $m_{r s}$ of $M$ is

$$
m_{r s}=a_{r s}+R \sum_{l \in \mathbb{Z}} \delta_{s, k+l n}\left(\delta_{r, h+l n}-\delta_{r, h+1+l n}\right)+R \sum_{l \in \mathbb{Z}} \delta_{s, n-k+l n}\left(\delta_{r, n-h+l n}-\delta_{r, n-1-h+l n}\right) .
$$


Observe that

$$
\begin{aligned}
& \sum_{r \leqslant i, s \geqslant j} R \sum_{l \in \mathbb{Z}} \delta_{s, k+l n}\left(\delta_{r, h+l n}-\delta_{r, h+1+l n}\right) \\
&= \begin{cases}R, & \text { if } i=h+l_{1} n, j \leqslant k+l_{1} n, \text { for some } l_{1}, \\
0, & \text { otherwise. }\end{cases} \\
& \sum_{r \leqslant i, s \geqslant j}+R \sum_{l \in \mathbb{Z}} \delta_{s, n-k+l n}\left(\delta_{r, n-h+l n}-\delta_{r, n-1-h+l n}\right) \\
&= \begin{cases}-R, & \text { if } i=n-1-h+l_{1} n, j \leqslant n-k+l_{1} n, \text { for some } l_{1}, \\
0, & \text { otherwise. }\end{cases}
\end{aligned}
$$

On the other hand, the $(r, s)$-th entry $m_{r, s}^{\prime}$ of $M^{\prime}$ is equal to

$$
m_{r s}^{\prime}=a_{r s}+\sum_{l \in \mathbb{Z}} t_{s-l n}\left(\delta_{r, h+l n}-\delta_{r, h+1+l n}\right)+\sum_{l \in \mathbb{Z}} t_{n-s+l n}\left(\delta_{r, n-h+l n}-\delta_{r, n-1-h+l n}\right) .
$$

Notice that

$$
\begin{aligned}
& \sum_{r \leqslant i, s \geqslant j} \sum_{l \in \mathbb{Z}} t_{s-l n}\left(\delta_{r, h+l n}-\delta_{r, h+1+l n}\right) \\
& = \begin{cases}\sum_{s+l_{1} n \geqslant j} t_{s}, & \text { if } i=h+l_{1} n, \text { for some } l_{1}, \\
0, & \text { otherwise. }\end{cases} \\
& \sum_{r \leqslant i, s \geqslant j} \sum_{l \in \mathbb{Z}} t_{n-s+l n}\left(\delta_{r, n-h+l n}-\delta_{r, n-1-h+l n}\right) \\
& = \begin{cases}-\sum_{n-s+l_{1} n \geqslant j} t_{s}, & \text { if } i=n-1-h+l_{1} n, \\
0, & \text { otherwise. }\end{cases}
\end{aligned}
$$

To show that $M^{\prime} \leqslant$ alg $M$ when $A$ is subject to the second condition, it suffices to show that (4.4.1) $\geqslant(4.4 .3)$ and (4.4.2) $\geqslant(4.4 .4)$ when $i<j$. Indeed, since $A$ satisfies the second condition, we have $t_{u}=0$ unless $u \leqslant k$. If $i<j$ and $i=h+l_{1} n$ for some $l_{1}$, we have $\sum_{s+l_{1} n \geqslant j} t_{s} \leqslant \sum_{s>h} t_{s} \leqslant R$. If, moreover, $h \geqslant k$, then $\sum_{s>h} t_{s} \leqslant \sum_{s>k} t_{s}=0$. From these data, we see that (4.4.3) $\leqslant$ (4.4.1) when $i<j$. When $i<j$, we see that (4.4.2) is equal to $-R$ when $j \leqslant n-k+l_{1} n$, and in this case (4.4.4) is also equal to $-R$. So we have (4.4.4) $\leqslant$ (4.4.2) when $i<j$. Therefore we have (1) when $A$ is subject to the second condition.

For $A$ subject to either of the remaining conditions, the proof of (1) is entirely similar and is left to the readers.

We now prove (2) for $h \in[1, r-1]$, i.e., when $A$ is subject to the second condition. Suppose that $M=A-R\left(E_{\theta}^{h k}-E_{\theta}^{h+1, k}\right)$ and $M^{\prime}=A-\sum_{u \in \mathbb{Z}} t_{u}\left(E_{\theta}^{h u}-E_{\theta}^{h+1, u}\right)$ with $\sum_{u \in \mathbb{Z}} t_{u}=R$. It suffices to show that $M^{\prime} \leqslant$ alg $M$. Similar to the proof of (1), it is reduced to show that (4.4.1) $\leqslant(4.4 .3)$ and $(4.4 .2) \leqslant(4.4 .4)$ when $i<j$. By assumption, we see that $t_{u}=0$ unless $u \geqslant k$. When (4.4.1) takes value $R$, then $j \leqslant k+l_{1} n$, which implies that $\sum_{s+l_{1} n \geqslant j} t_{s}=\sum_{s \geqslant k} t_{s}=R$. Hence (4.4.1) $\leqslant(4.4 .3)$ in this case. When (4.4.2) takes value 0 , we have either $j>n-k+l_{1} n$ for some $l_{1}$ or $i \neq n-1-h+l_{1} n$ for any $l_{1}$. For the latter case, (4.4.4) is always zero. For the former, we have $\sum_{n-s+l_{1} n} t_{s}=\sum_{s<k} t_{s}=0$. Thus we have (4.4.2) $\leqslant$ (4.4.4). Therefore we have proved (2) if $A$ satisfies the second condition. 
For the remaining cases, the proof of (2) is again similar and skipped.

The following lemma is the counterpart of Lemma 2.2.2.

Lemma 4.4.2. Let $A, B, C \in \Xi_{n, d}$. Let $R$ be a positive integer.

(1) Assume that $B-R E_{\theta}^{h, h+1}$ is diagonal for some $h \in[0, r]$ and $\operatorname{co}(B)=\operatorname{ro}(A)$. Assume further that $R=R_{0}+\cdots+R_{l}$ and the matrix $A$ satisfy one of the following conditions:

$$
\begin{cases}a_{0 m}=0, a_{1, k+i}=R_{i}, a_{1 k} \geqslant R_{0}, a_{1 j}=0, & \text { if } h=0, k \geqslant 1 ; \\ a_{h m}=0, a_{h+1, k+i}=R_{i}, a_{h+1, k} \geqslant R_{0}, a_{h+1, j}=0, & \text { if } h \in[1, r-1] ; \\ a_{r m}=0, a_{r+1, k+i}=R_{i}, a_{r+1, k} \geqslant R_{0}, a_{r+1, j}=0, & \text { if } h=r, k>r+1 ; \\ a_{r m}=0, a_{r+1, k+i}=R_{i}, a_{r+1, k} \geqslant 2 R_{0}, a_{r+1, j}=0, & \text { if } h=r, k=r+1\end{cases}
$$

for all $m \geqslant k, i \in[1, l]$ and $j>k+l$. Then we have

$$
[B] *[A]=\left[A+\sum_{i=0}^{l} R_{i}\left(E_{\theta}^{h, k+i}-E_{\theta}^{h+1, k+i}\right)\right]+\text { lower terms. }
$$

(2) Assume that $C-R E_{\theta}^{h+1, h}$ is diagonal for some $h \in[0, r]$ and $\operatorname{co}(C)=\operatorname{ro}(A)$. Assume further that $R=R_{0}+\cdots+R_{l}$ and $A$ satisfy one of the following conditions:

$$
\begin{cases}a_{1 m}=0, a_{0, k+i}=R_{i}, a_{0, k+l} \geqslant R_{l}, a_{0 j}=0, & \text { if } h=0, k+l<0 ; \\ a_{1 m}=0, a_{0, k+i}=R_{i}, a_{00} \geqslant 2 R_{l}, a_{0 j}=0, & \text { if } h=0, k+l=0 ; \\ a_{h+1, m}=0, a_{h, k+i}=R_{i}, a_{h, k+l} \geqslant R_{l}, a_{h j}=0, & \text { if } h \in[1, r-1] \\ a_{r+1, m}=0, a_{r, k+i}=R_{i}, a_{r, k+l} \geqslant R_{l}, a_{r j}=0, & \text { if } h=r, k<r .\end{cases}
$$

for all $m \leqslant k+l, i \in[0, l-1]$ and $j<k$. Then we have

$$
[C] *[A]=\left[A-\sum_{i=0}^{l} R_{i}\left(E_{\theta}^{h, k+i}-E_{\theta}^{h+1, k+i}\right)\right]+\text { lower terms. }
$$

Proof. We show (1). By a similar argument as that for Lemma 4.4.1, the leading term is $\left[A+\sum_{i=0}^{l} R_{i}\left(E_{\theta}^{h, k+i}-E_{\theta}^{h+1, k+i}\right)\right]$. It remains to show that its coefficient is 1. In this case, we have

$$
t_{k+i}=R_{i}, \forall i \in[0, l], \quad \text { and } \quad t_{j}=0, \forall j \notin[k, k+l] .
$$

By Proposition 4.3.3, we have

$$
\begin{cases}\prod_{u>0} \overline{\left[\begin{array}{c}
a_{0 u}+t_{u}+t_{-u} \\
t_{u}
\end{array}\right]} \prod_{u<0} \overline{\left[\begin{array}{c}
a_{0 u}+t_{u} \\
t_{u}
\end{array}\right]} \prod_{i=0}^{t_{0}-1 \overline{\frac{\left[a_{00}+1+2 i\right]}{\overline{[i+1]}}}=1,} & \text { if } h=0, \\
\prod_{u \in \mathbb{Z}} \overline{\left[\begin{array}{c}
\left.a_{h u}+t_{u}\right] \\
t_{u}
\end{array}\right]=1,} & \text { if } h \neq 0 .\end{cases}
$$

Moreover, we have

$$
\begin{cases}\beta_{t}=\sum_{j>u}\left(t_{j}-a_{h+1, j}\right) t_{u}+\frac{1}{2}\left(\sum_{j+u<n+1} t_{j} t_{u}-\sum_{j<r+1} t_{j}\right)=0, & \text { if } h \neq 0, \\ \beta_{t}^{\prime}=-\sum_{j>u} a_{1 j} t_{u}-\sum_{j>0} \frac{t_{j}^{2}-t_{j}}{2}+\frac{R^{2}-R}{2}=\frac{1}{2}\left(R^{2}-\sum_{j} t_{j}^{2}-2 \sum_{j>u} t_{j} t_{u}\right)=0, & \text { if } h=0 .\end{cases}
$$

In each case, the leading coefficient is 1 , and whence (1). A similar proof of (2) is skipped. 
CHAPTER 5

\section{Coideal algebra type structures of Schur algebras and Lusztig algebras}

In this chapter, we formulate a coideal algebra type structure which involves Schur algebras of both affine type $C$ and $A$, and its behavior on the Chevalley generators. This leads to an imbedding $\jmath_{n, d}$ from $\mathbf{S}_{n, d}^{\mathfrak{c}}$ to $\mathbf{S}_{n, d}$ (Schur algebra of affine type $A$ ). We show the comultiplication and $\jmath_{n, d}$ behave well when replacing Schur algebras by Lusztig subalgebras. The canonical bases and monomial bases are shown to be compatible under the inclusion $\mathbf{U}_{n, d}^{\mathfrak{c}} \subset \mathbf{S}_{n, d}^{\mathfrak{c}}$.

\subsection{The Lusztig Algebra $\mathbf{U}_{n, d}^{\mathfrak{c}}$}

We now set

$$
n=2 r+2, \quad(r \in \mathbb{N}) .
$$

Recall the Schur algebra $\mathbf{S}_{n, d}^{\mathfrak{c}}$ from (4.2.8). Let $\mathbf{U}_{n, d}^{\mathfrak{c}}$ be the subalgebra of $\mathbf{S}_{n, d}^{\mathfrak{c}}$ generated by all elements $[B]$ such that $B, B-E_{\theta}^{h, h+1}$ or $B-E_{\theta}^{h+1, h}$ is diagonal for various $h$. Let $\mathbf{U}_{n, d ; \mathcal{A}}^{\mathfrak{c}}$ denote the $\mathcal{A}$-subalgebra of $\mathbf{S}_{n, d}^{\mathfrak{c}}$ generated by all elements [B] such that $B, B-R E_{\theta}^{h, h+1}$ or $B-R E_{\theta}^{h+1, h}$ is diagonal for various $h$ and $R \in \mathbb{N}$. Following the affine type $A$ setting, we make the following definition.

Definition 5.1.1. The algebra $\mathbf{U}_{n, d}^{\mathfrak{c}}$ is called the Lusztig algebra (of affine type $C$ ).

For $i \in[0, r]$ and $a \in[-1, r+1]$, we define the following functions (with the notation $\stackrel{1}{\subset}, \stackrel{1}{\supset}$ denoting inclusions of codimension 1 and $|W|$ for the dimension of a $k$-vector space): for any $L=\left(L_{i}\right)_{i \in \mathbb{Z}}, L^{\prime}=\left(L_{i}^{\prime}\right)_{i \in \mathbb{Z}} \in \mathcal{X}_{n, d}^{\mathfrak{c}}$

$$
\begin{aligned}
\mathbf{e}_{i}\left(L, L^{\prime}\right) & = \begin{cases}v^{-\left|L_{i+1}^{\prime} / L_{i}^{\prime}\right|-\delta_{i, r},} & \text { if } L_{i} \stackrel{1}{\subset} L_{i}^{\prime}, L_{j}=L_{j^{\prime}}, \forall j \in[0, r] \backslash\{i\} ; \\
0, & \text { otherwise. }\end{cases} \\
\mathbf{f}_{i}\left(L, L^{\prime}\right) & = \begin{cases}v^{-\left|L_{i}^{\prime} / L_{i-1}^{\prime}\right|-\delta_{i, 0}}, & \text { if } L_{i} \stackrel{1}{\supset} L_{i}^{\prime}, L_{j}=L_{j}^{\prime}, \forall j \in[0, r] \backslash\{i\} ; \\
0, & \text { otherwise. }\end{cases} \\
\mathbf{h}_{a}^{ \pm 1}\left(L, L^{\prime}\right) & =v^{ \pm\left(\left|L_{a}^{\prime} / L_{a-1}^{\prime}\right|+\delta_{a, 0}+\delta_{a, r+1}\right)} \delta_{L, L^{\prime}} . \\
\mathbf{k}_{i} & =\mathbf{h}_{i+1} \mathbf{h}_{i}^{-1} .
\end{aligned}
$$

It follows by the definition that for $i \in[0, r]$ and $a \in[0, r+1]$,

$$
\mathbf{e}_{i}=\sum[A], \quad \mathbf{f}_{i}=\sum[A], \quad \mathbf{h}_{a}=\sum_{\lambda \in \Lambda_{n, d}^{\mathfrak{c}}} v^{\lambda_{a}} 1_{\lambda}, \quad \mathbf{k}_{i}=\sum_{\lambda \in \Lambda_{n, d}^{\mathrm{c}}} v^{\lambda_{i+1}-\lambda_{i}} 1_{\lambda},
$$


where the first two sums run over all $A \in \Xi_{n, d}$ such that $A-E_{\theta}^{i+1, i}$ and $A-E_{\theta}^{i, i+1}$ are diagonal, respectively, $1_{\lambda}$ stands for the standard basis element of a diagonal matrix whose diagonal is $\lambda$, and

$$
\Lambda_{n, d}^{\mathfrak{c}}=\left\{\left(a_{i}\right)_{i \in \mathbb{Z}} \mid a_{i} \in \mathbb{N}, a_{i}=a_{-i}, a_{i}=a_{n-i}, \sum_{1 \leqslant i \leqslant n} a_{i}=2 d+2, a_{0}, a_{r+1} \text { odd }\right\} .
$$

So we have $\mathbf{e}_{i}, \mathbf{f}_{i}, \mathbf{k}_{i}^{ \pm 1}, \mathbf{h}_{a}^{ \pm 1} \in \mathbf{U}_{n, d}^{\mathfrak{c}}$.

By the local property of $L_{0}$ in Section 3.3, one can obtain the following relations (5.1.6) by using a similar argument in [BKLW14, Proposition 3.1] for the relations related to the generators $\mathbf{e}_{r}, \mathbf{f}_{r}, \mathbf{h}_{r}$ and $\mathbf{h}_{r+1}$ for $r \geqslant 1$. Note that the generators $\mathbf{e}_{0}, \mathbf{f}_{0}, \mathbf{h}_{0}$ and $\mathbf{h}_{1}$ play the roles of the respective generators $\mathbf{f}_{r}, \mathbf{e}_{r}, \mathbf{h}_{r+1}$ and $\mathbf{h}_{r}$ in the argument, by comparing items (1) and (2) in Lemma 3.3.3. For $r \geqslant 1$, we have

$$
\begin{aligned}
\mathbf{h}_{0} \mathbf{f}_{0} & =v^{2} \mathbf{f}_{0} \mathbf{h}_{0}, \quad \mathbf{h}_{0} \mathbf{e}_{0}=v^{-2} \mathbf{e}_{0} \mathbf{h}_{0}, \\
\mathbf{e}_{0}^{2} \mathbf{f}_{0}+\mathbf{f}_{0} \mathbf{e}_{0}^{2} & =\left(v+v^{-1}\right)\left(\mathbf{e}_{0} \mathbf{f}_{0} \mathbf{e}_{0}-\left(v \mathbf{h}_{1}^{-1} \mathbf{h}_{0}+v^{-1} \mathbf{h}_{1} \mathbf{h}_{0}^{-1}\right) \mathbf{e}_{0}\right), \\
\mathbf{f}_{0}^{2} \mathbf{e}_{0}+\mathbf{e}_{0} \mathbf{f}_{0}^{2} & =\left(v+v^{-1}\right)\left(\mathbf{f}_{0} \mathbf{e}_{0} \mathbf{f}_{0}-\mathbf{f}_{0}\left(v \mathbf{h}_{1}^{-1} \mathbf{h}_{0}+v^{-1} \mathbf{h}_{1} \mathbf{h}_{0}^{-1}\right)\right) .
\end{aligned}
$$

For $i, j \in[0, r]$, we denote the Cartan integers by

$$
\mathrm{c}_{i j}=2 \delta_{i j}-\delta_{i, j+1}-\delta_{i, j-1} .
$$

Proposition 5.1.2. Let $r \geqslant 1$. The elements $\mathbf{e}_{i}, \mathbf{f}_{i}$, and $\mathbf{k}_{i}^{ \pm 1}$ for $i \in[0, r]$ satisfy the following relations in $\mathbf{U}_{n, d}^{\mathfrak{c}}$, for all $i, j \in[0, r]$ :

$$
\begin{aligned}
\mathbf{k}_{0}\left(\mathbf{k}_{1}^{2} \cdots \mathbf{k}_{r-1}^{2}\right) \mathbf{k}_{r}=1, \\
\mathbf{k}_{i} \mathbf{k}_{i}^{-1}=1, \quad \mathbf{k}_{i} \mathbf{k}_{j}=\mathbf{k}_{j} \mathbf{k}_{i}, \\
\mathbf{k}_{i} \mathbf{e}_{j} \mathbf{k}_{i}^{-1}=v^{c_{i j}+\delta_{i, 0} \delta_{j, 0}+\delta_{i, r} \delta_{j, r}} \mathbf{e}_{j}, \\
\mathbf{k}_{i} \mathbf{f}_{j} \mathbf{k}_{i}^{-1}=v^{-c_{i j}-\delta_{i, 0} \delta_{j, 0}-\delta_{i, r} \delta_{j, r}} \mathbf{f}_{j}, \\
\mathbf{e}_{i} \mathbf{e}_{j}=\mathbf{e}_{j} \mathbf{e}_{i}, \quad \mathbf{f}_{i} \mathbf{f}_{j}=\mathbf{f}_{j} \mathbf{f}_{i}, \quad \forall|i-j|>1, \\
\mathbf{e}_{i}^{2} \mathbf{e}_{j}+\mathbf{e}_{j} \mathbf{e}_{i}^{2}=\left(v+v^{-1}\right) \mathbf{e}_{i} \mathbf{e}_{j} \mathbf{e}_{i}, \quad \forall|i-j|=1, \\
\mathbf{f}_{i}^{2} \mathbf{f}_{j}+\mathbf{f}_{j} \mathbf{f}_{i}^{2}=\left(v+v^{-1}\right) \mathbf{f}_{i} \mathbf{f}_{j} \mathbf{f}_{i}, \quad \forall|i-j|=1, \\
\mathbf{e}_{i} \mathbf{f}_{j}-\mathbf{f}_{j} \mathbf{e}_{i}=\delta_{i j} \frac{\mathbf{k}_{i}-\mathbf{k}_{i}^{-1}}{v-v^{-1}}, \quad \forall(i, j) \neq(0,0),(r, r), \\
\mathbf{e}_{0}^{2} \mathbf{f}_{0}+\mathbf{f}_{0} \mathbf{e}_{0}^{2}=\left(v+v^{-1}\right)\left(\mathbf{e}_{0} \mathbf{f}_{0} \mathbf{e}_{0}-\left(v \mathbf{k}_{0}+v^{-1} \mathbf{k}_{0}^{-1}\right) \mathbf{e}_{0}\right), \\
\mathbf{e}_{r}^{2} \mathbf{f}_{r}+\mathbf{f}_{r} \mathbf{e}_{r}^{2}=\left(v+v^{-1}\right)\left(\mathbf{e}_{r} \mathbf{f}_{r} \mathbf{e}_{r}-\mathbf{e}_{r}\left(v \mathbf{k}_{r}+v^{-1} \mathbf{k}_{r}^{-1}\right)\right), \\
\mathbf{f}_{0}^{2} \mathbf{e}_{0}+\mathbf{e}_{0} \mathbf{f}_{0}^{2}=\left(v+v^{-1}\right)\left(\mathbf{f}_{0} \mathbf{e}_{0} \mathbf{f}_{0}-\mathbf{f}_{0}\left(v \mathbf{k}_{0}+v^{-1} \mathbf{k}_{0}^{-1}\right)\right), \\
\mathbf{f}_{r}^{2} \mathbf{e}_{r}+\mathbf{e}_{r} \mathbf{f}_{r}^{2}=\left(v+v^{-1}\right)\left(\mathbf{f}_{r} \mathbf{e}_{r} \mathbf{f}_{r}-\left(v \mathbf{k}_{r}+v^{-1} \mathbf{k}_{r}^{-1}\right) \mathbf{f}_{r}\right) .
\end{aligned}
$$

Proof. In light of (5.1.6) the verification of the relations is essentially reduced to the finite type computations, which is given in [BKLW14, Proposition 3.1]. We skip the detail.

The following lemma is an analogue of [BKLW14, Corollary 3.13] which follows by a standard Vandermonde determinant type argument. 
Lemma 5.1.3. The algebra $\mathbf{U}_{n, d}^{\mathfrak{c}}$ is generated by $\mathbf{e}_{i}, \mathbf{f}_{i}$ and $\mathbf{k}_{i}^{ \pm 1}$ for all $i \in[0, r]$.

We will refer to the generators of the algebra $\mathbf{U}_{n, d}^{\mathfrak{c}}$ given by the above lemma as Chevalley generators.

\subsection{A RAW COMULtiplication}

In this section, we shall give the definition of a raw comultiplication $\widetilde{\Delta}^{\mathfrak{c}}$, which is a key component in the construction of a refined comultiplication $\Delta^{\mathfrak{c}}$ in Section 5.3 .

The raw comultiplication is originally defined by Lusztig in [Lu00] (though this terminology was not used therein), which has geometric origin, while it is incompatible with algebraic comultiplication in literature. To prove the positivity of the algebraic comultiplication, in [FL15, the raw comultiplication is modified to be the one, called refined comultiplication, which is compatible with its algebraic definition. This construction is generalized to affine type C setting in Section 5.3 .

We fix some notations to begin with. Let $k=\mathbb{F}_{q}, F=k((\varepsilon))$, and $\mathfrak{o}=k[[\varepsilon]]$ where $q$ is odd. Let $V$ be a symplectic $F$-vector space of dimension $2 d$ with the form $(-,-)$. Let $V^{\prime \prime}$ be an isotropic $F$-subspace of $V$ of dimension $d^{\prime \prime}$, and so $V^{\prime}=V^{\prime \prime} \perp / V^{\prime \prime}$ is a symplectic space of dimension $2 d^{\prime}$ with its symplectic form induced from $V$; note that $d^{\prime}=d-d^{\prime \prime}$.

Given a periodic chain $L$ in $\mathcal{X}_{n, d}^{\mathfrak{c}}$, we can define a periodic chain $L^{\prime \prime}:=\pi^{\prime \prime}(L) \in \mathcal{X}_{n, d^{\prime \prime}}$ (of affine type $A$ ) by setting $L_{i}^{\prime \prime}=L_{i} \cap V^{\prime \prime}$ for all $i$. We can also define a periodic chain $L^{\prime}=\pi^{\natural}(L) \in \mathcal{X}_{n, d^{\prime}}^{\mathfrak{c}}$ by setting $L_{i}^{\prime}=\left(L_{i} \cap V^{\prime \prime \perp}+V^{\prime \prime}\right) / V^{\prime \prime}$ for all $i$. Given any pair $\left(L^{\prime}, L^{\prime \prime}\right) \in$ $\mathcal{X}_{n, d^{\prime}}^{\mathfrak{c}} \times \mathcal{X}_{n, d^{\prime \prime}}$, we set

$$
\mathcal{Z}_{L^{\prime}, L^{\prime \prime}}^{\mathfrak{c}}=\left\{L \in \mathcal{X}_{n, d}^{\mathfrak{c}} \mid \pi^{\natural}(L)=L^{\prime}, \pi^{\prime \prime}(L)=L^{\prime \prime}\right\}
$$

We can define a map

$$
\widetilde{\Delta}^{\mathfrak{c}}: \mathbf{S}_{n, d}^{\mathfrak{c}} \longrightarrow \mathbf{S}_{n, d^{\prime}}^{\mathfrak{c}} \otimes \mathbf{S}_{n, d^{\prime \prime}}, \quad \forall d^{\prime}+d^{\prime \prime}=d,
$$

such that, when specializing the parameter $v$ at $v=\sqrt{q}$, it is given by

$$
\widetilde{\Delta}^{\mathfrak{c}}(f)\left(L^{\prime}, \tilde{L}^{\prime}, L^{\prime \prime}, \tilde{L}^{\prime \prime}\right)=\sum_{\tilde{L} \in \mathcal{Z}_{\tilde{L}^{\prime}, \tilde{L}^{\prime \prime}}} f(L, \tilde{L}), \quad \forall L^{\prime}, \tilde{L}^{\prime} \in \mathcal{X}_{n, d^{\prime}}^{\mathfrak{c}}, L^{\prime \prime}, \tilde{L}^{\prime \prime} \in \mathcal{X}_{n, d^{\prime \prime}},
$$

where $L$ is a fixed element in $\mathcal{Z}_{L^{\prime}, L^{\prime \prime}}^{\mathrm{c}}$. Note the appearance of $\mathbf{S}_{n, d^{\prime \prime}}$ in (5.2.1), which is an Schur algebra of affine type $A$ defined in (2.2.3).

By applying Proposition 3.1.2, we have the following analogue of [Lu00, Lemma 1.3].

Lemma 5.2.1. Suppose that $V^{\prime \prime}$ is an isotropic subspace of the symplectic space $V$ and $L=\left(L_{i}\right)_{i \in \mathbb{Z}} \in \mathcal{X}_{n, d}^{\mathfrak{c}}$. Then we can find a pair $(T, W)$ of subspaces in $V$ such that

(1) $V=V^{\prime \prime} \oplus T \oplus W,\left(V^{\prime \prime}\right)^{\perp}=V^{\prime \prime} \oplus T$,

(2) $W$ is isotropic, $(T, W)=0$,

(3) There exist bases $\left\{z_{1}, \ldots, z_{s}\right\}$ and $\left\{w_{1}, \ldots, w_{s}\right\}$ of $V^{\prime \prime}$ and $W$, respectively, such that $\left(z_{i}, w_{j}\right)=\delta_{i j}$ for any $i, j \in[1, s]$,

(4) $L_{i}=\left(L_{i} \cap V^{\prime \prime}\right) \oplus\left(L_{i} \cap T\right) \oplus\left(L_{i} \cap W\right)$, for any $i \in \mathbb{Z}$.

We can now show that the definition (5.2.2) is well defined (i.e., it is independent of the choice of $L$ ), following the argument in [Lu00, 1.2]; see also [FL15, 3.2]. For fixed $L \in \mathcal{X}_{n, d}^{\mathfrak{c}}$, let $\tilde{\mathcal{Z}}_{L}^{\mathfrak{c}}$ be the set of all pairs $(T, W)$ satisfying the first three conditions in Lemma 5.2.1, 
Note that given a pair $(T, W)$ in $\tilde{\mathcal{Z}}_{L}^{\mathfrak{c}}$, we have an isomorphism $\pi: T \rightarrow V^{\prime \prime \perp} / V^{\prime \prime}$. Now if $L \in \mathcal{Z}_{L^{\prime}, L^{\prime \prime}}^{\mathfrak{c}}$, we define a map

$$
\psi: \tilde{\mathcal{Z}}_{L}^{\mathfrak{c}} \rightarrow \mathcal{Z}_{L^{\prime}, L^{\prime \prime}}^{\mathfrak{c}}, \quad(T, W) \mapsto L^{T, W}
$$

where

$$
L_{i}^{T, W}=L_{i}^{\prime \prime} \oplus \pi^{-1}\left(L_{i}^{\prime}\right) \oplus\left(L_{-i-1}^{\prime \prime}\right)_{W}^{\#}, \quad\left(L_{-i-1}^{\prime \prime}\right)_{W}^{\#}=\left\{w \in W \mid\left(w, L_{-i-1}^{\prime \prime}\right) \in \mathfrak{o}\right\}, \quad \forall i \in \mathbb{Z} .
$$

By Lemma 5.2.1, the map $\psi$ is surjective.

Let $P_{V^{\prime \prime}}$ be the stabilizer of the flag $V^{\prime \prime} \subseteq V^{\prime \prime} \perp$ in $\operatorname{Sp}_{F}(V)$. Let $\mathcal{U}=\mathcal{U}_{V^{\prime \prime}}$ be its unipotent radical, i.e., the set of all $g \in \operatorname{Sp}_{F}(V)$ such that $g(x)=x$ for all $x \in V^{\prime \prime}$ and $g(y)-y \in V^{\prime \prime}$ for all $y \in V^{\prime \prime \perp}$. The $\operatorname{Sp}_{F}(V)$-actions on $\mathcal{X}_{n, d}^{\mathfrak{c}}$ and $V$ restrict to the $\mathcal{U}$-actions on $\tilde{\mathcal{Z}}_{L}^{\mathfrak{c}}$ and $\mathcal{Z}_{L^{\prime}, L^{\prime \prime}}^{\mathfrak{c}}$, respectively. Clearly, $\psi$ is $\mathcal{U}$-equivariant and $\mathcal{U}$ acts transitively on $\tilde{Z}_{L}^{\mathfrak{c}}$, and so $\mathcal{U}$ acts transitively on $\mathcal{Z}_{L^{\prime}, L^{\prime \prime}}^{\mathfrak{c}}$. This means that if $\hat{L} \in \mathcal{Z}_{L^{\prime}, L^{\prime \prime}}^{\mathfrak{c}}$, there is $g \in \mathcal{U}$ such that $g \hat{L}=L$. From this, we have for all $\hat{L} \in \mathcal{Z}_{L^{\prime}, L^{\prime \prime}}^{\mathfrak{c}}$,

$$
\sum_{\tilde{L} \in \mathcal{Z}_{\tilde{L}^{\prime}, \tilde{L}^{\prime \prime}}^{\mathfrak{c}}} f(\hat{L}, \tilde{L})=\sum_{\tilde{L} \in \mathcal{Z}_{\tilde{L}^{\prime}, \tilde{L}^{\prime \prime}}^{\mathfrak{c}}} f\left(L, g^{-1} \tilde{L}\right)=\sum_{\tilde{L} \in \mathcal{Z}_{\tilde{L}^{\prime}, \tilde{L}^{\prime \prime}}^{\mathfrak{c}}} f(L, \tilde{L}) .
$$

Therefore the definition of (5.2.2) and hence $\tilde{\Delta}^{\mathfrak{c}}$ is independent of the choice of $L$.

Following the argument of [Lu00, Proposition 1.5], which is formal and not reproduced here, we have the following proposition.

Proposition 5.2.2. The map $\widetilde{\Delta}^{\mathfrak{c}}$ is an algebra homomorphism.

Now we determine how the map $\widetilde{\Delta}^{\mathfrak{c}}$ acts on the generators. Recall from Chapter 2 the Chevalley generators $\mathbf{H}_{i}, \mathbf{E}_{i}$ and $\mathbf{F}_{i}$ for Lusztig algebra $\mathbf{U}_{n, d}$ of affine type $A$ (a subalgebra of the Schur algebra $\mathbf{S}_{n, d}$ of affine type $A$ ), and that $\mathbf{H}_{n+i}=\mathbf{H}_{i}, \mathbf{E}_{n+i}=\mathbf{E}_{i}$ and $\mathbf{F}_{n+i}=\mathbf{F}_{i}$.

Proposition 5.2.3. For any $i \in[0, r]$, we have

$$
\begin{aligned}
\widetilde{\Delta}^{\mathfrak{c}}\left(\mathbf{e}_{i}\right) & =\mathbf{e}_{i}^{\prime} \otimes \mathbf{H}_{i+1}^{\prime \prime} \mathbf{H}_{n-1-i}^{\prime \prime-1}+\mathbf{h}_{i+1}^{\prime-1} \otimes \mathbf{E}_{i}^{\prime \prime} \mathbf{H}_{n-1-i}^{\prime \prime-1}+\mathbf{h}_{i+1}^{\prime} \otimes \mathbf{F}_{n-1-i}^{\prime \prime} \mathbf{H}_{i+1}^{\prime \prime} . \\
\widetilde{\Delta}^{\mathfrak{c}}\left(\mathbf{f}_{i}\right) & =\mathbf{f}_{i}^{\prime} \otimes \mathbf{H}_{i}^{\prime \prime-1} \mathbf{H}_{n-i}^{\prime \prime}+\mathbf{h}_{i}^{\prime} \otimes \mathbf{F}_{i}^{\prime \prime} \mathbf{H}_{n-i}^{\prime \prime}+\mathbf{h}_{i}^{\prime-1} \otimes \mathbf{E}_{n-1-i}^{\prime \prime} \mathbf{H}_{i}^{\prime \prime-1} . \\
\widetilde{\Delta}^{\mathfrak{c}}\left(\mathbf{k}_{i}\right) & =\mathbf{k}_{i}^{\prime} \otimes \mathbf{K}_{i}^{\prime \prime} \mathbf{K}_{n-1-i}^{\prime \prime-1} .
\end{aligned}
$$

Here the superscripts ' and "indicate that the underlying Chevalley generators lie in $\mathbf{S}_{n, d^{\prime}}^{\mathfrak{c}}$ and $\mathbf{S}_{n, d^{\prime \prime}}$, respectively.

Proof. For any $L \in \mathcal{X}_{n, d}^{\mathfrak{c}}$, we have

$$
\left|L_{i+1} / L_{i}\right|=\left|L_{i+1}^{\prime} / L_{i}^{\prime}\right|+\left|L_{i+1}^{\prime \prime} / L_{i}^{\prime \prime}\right|+\left|L_{n-1-i}^{\prime \prime} / L_{n-2-i}^{\prime \prime}\right| .
$$

The proposition in the cases for $i \in[1, r]$ follows directly from Proposition A.3.2 for the finite type; also cf. [FL15. The case for $i=0$ follows from a similar argument to that of the case for $i=r$. Note that when $r=0$, one uses the non-degenerate symplectic form on $L_{1} / L_{0}=\varepsilon^{-1} L_{0}^{\#} / L_{0}$, inherited from that of $V$ (see [Lu03]). 


\subsection{The COMUltiplication $\Delta^{\mathfrak{c}}$}

Recall $\Lambda_{n, d}$ and $\Lambda_{n, d}^{\mathfrak{c}}$ from (2.2.2) and (5.1.5), respectively. The set $\mathcal{X}_{n, d}$ can be decomposed as follows:

$$
\mathcal{X}_{n, d}=\bigsqcup_{\mathbf{a}=\left(a_{i}\right) \in \Lambda_{n, d}} \mathcal{X}_{n, d}(\mathbf{a}), \quad \text { where } \mathcal{X}_{n, d}(\mathbf{a})=\left\{V \in \mathcal{X}_{n, d}|| V_{i} / V_{i-1} \mid=a_{i}, \forall 1 \leqslant i \leqslant n\right\} .
$$

Similarly the set $\mathcal{X}_{n, d}^{\mathfrak{c}}$ admits the following decomposition:

$\mathcal{X}_{n, d}^{\mathfrak{c}}=\bigsqcup_{\mathbf{a}=\left(a_{i}\right) \in \Lambda_{n, d}^{\mathfrak{c}}} \mathcal{X}_{n, d}^{\mathfrak{c}}(\mathbf{a}), \quad$ where $\mathcal{X}_{n, d}^{\mathfrak{c}}(\mathbf{a})=\left\{V \in \mathcal{X}_{n, d}^{\mathfrak{c}}|| V_{i} / V_{i-1} \mid=a_{i}-\delta_{i, r+1}-\delta_{i, n}, \forall 1 \leqslant i \leqslant n\right\}$.

Given $\mathbf{a}, \mathbf{b} \in \Lambda_{n, d}^{\mathfrak{c}}$, let $\mathbf{S}_{n, d}^{\mathfrak{c}}(\mathbf{b}, \mathbf{a})$ be the subspace of $\mathbf{S}_{n, d}^{\mathfrak{c}}$ spanned by the standard basis elements $[A]$ such that $\operatorname{ro}(A)=\mathbf{b}$ and $\operatorname{co}(A)=\mathbf{a}$. Similarly, for $\mathbf{a}, \mathbf{b} \in \Lambda_{n, d}$, we define the

affine type $A$ counterpart $\mathbf{S}_{n, d}(\mathbf{b}, \mathbf{a})$. Let $\widetilde{\Delta}_{\mathbf{b}^{\prime}, \mathbf{a}^{\prime}, \mathbf{b}^{\prime \prime}, \mathbf{a}^{\prime \prime}}^{\mathfrak{c}}$ be the component of $\widetilde{\Delta}^{\mathfrak{c}}$ from $\mathbf{S}_{n, d}^{\mathfrak{c}}(\mathbf{b}, \mathbf{a})$ to $\mathbf{S}_{n, d^{\prime}}^{\mathfrak{c}}\left(\mathbf{b}^{\prime}, \mathbf{a}^{\prime}\right) \otimes \mathbf{S}_{n, d^{\prime \prime}}\left(\mathbf{b}^{\prime \prime}, \mathbf{a}^{\prime \prime}\right)$ such that $b_{i}=b_{i}^{\prime}+b_{i}^{\prime \prime}+b_{-i}^{\prime \prime}, a_{i}=a_{i}^{\prime}+a_{i}^{\prime \prime}+a_{-i}^{\prime \prime}$, for $i \in \mathbb{Z}$. We set

$$
s\left(\mathbf{b}^{\prime}, \mathbf{a}^{\prime}, \mathbf{b}^{\prime \prime}, \mathbf{a}^{\prime \prime}\right)=\sum_{1 \leqslant k \leqslant j \leqslant n} b_{k}^{\prime} b_{j}^{\prime \prime}-a_{k}^{\prime} a_{j}^{\prime \prime},
$$

and

$$
u\left(\mathbf{b}^{\prime \prime}, \mathbf{a}^{\prime \prime}\right)=\frac{1}{2}\left(\sum_{\substack{1 \leqslant k, j \leqslant n-1 \\ k+j \geqslant n}} b_{k}^{\prime \prime} b_{j}^{\prime \prime}-a_{k}^{\prime \prime} a_{j}^{\prime \prime}+\sum_{n-1 \geqslant k \geqslant r+1} a_{k}^{\prime \prime}-b_{k}^{\prime \prime}\right),
$$

for all $\mathbf{b}^{\prime}, \mathbf{a}^{\prime} \in \Lambda_{n, d^{\prime}}^{\mathfrak{c}}$ and $\mathbf{b}^{\prime \prime}, \mathbf{a}^{\prime \prime} \in \Lambda_{n, d^{\prime \prime}}$. We renormalize the raw comultiplication $\widetilde{\Delta}^{\mathfrak{c}}$ to be $\widetilde{\Delta}^{\mathfrak{c} \dagger}$ by letting

$$
\begin{aligned}
\Delta_{\mathbf{b}^{\prime}, \mathbf{a}^{\prime}, \mathbf{b}^{\prime \prime}, \mathbf{a}^{\prime \prime}}^{\mathfrak{c} \dagger} & =v^{s\left(\mathbf{b}^{\prime}, \mathbf{a}^{\prime}, \mathbf{b}^{\prime \prime}, \mathbf{a}^{\prime \prime}\right)+u\left(\mathbf{b}^{\prime \prime}, \mathbf{a}^{\prime \prime}\right)} \widetilde{\Delta}_{\mathbf{b}^{\prime}, \mathbf{a}^{\prime}, \mathbf{b}^{\prime \prime}, \mathbf{a}^{\prime \prime}}^{\mathfrak{c}}, \\
\Delta^{\mathfrak{c} \dagger} & =\bigoplus_{\mathbf{b}^{\prime}, \mathbf{a}^{\prime}, \mathbf{b}^{\prime \prime}, \mathbf{a}^{\prime \prime}} \Delta_{\mathbf{b}^{\prime}, \mathbf{a}^{\prime}, \mathbf{b}^{\prime \prime}, \mathbf{a}^{\prime \prime}}^{\mathfrak{c} \dagger}: \mathbf{S}_{n, d}^{\mathfrak{c}} \rightarrow \mathbf{S}_{n, d^{\prime}}^{\mathfrak{c}} \otimes \mathbf{S}_{n, d^{\prime \prime}}
\end{aligned}
$$

Proposition 5.3.1. Let $d=d^{\prime}+d^{\prime \prime}$. For all $i \in[0, r]$, we have

$$
\begin{aligned}
\Delta^{\mathfrak{c} \dagger}\left(\mathbf{e}_{i}\right) & =v^{\delta_{i, 0} d^{\prime \prime}} \mathbf{e}_{i}^{\prime} \otimes \mathbf{K}_{i}^{\prime \prime}+1 \otimes v^{-\delta_{i, 0}\left(2 d^{\prime}+2\right)} \mathbf{E}_{i}^{\prime \prime}+\mathbf{k}_{i}^{\prime} \otimes v^{\delta_{i, 0}\left(d^{\prime \prime}-1\right)+\delta_{i, 0}} \mathbf{F}_{n-1-i}^{\prime \prime} \mathbf{K}_{i}^{\prime \prime} . \\
\Delta^{\mathfrak{c} \dagger}\left(\mathbf{f}_{i}\right) & =v^{-\delta_{i, 0} d^{\prime \prime}} \mathbf{f}_{i}^{\prime} \otimes \mathbf{K}_{n-1-i}^{\prime \prime}+\mathbf{k}_{i}^{\prime-1} \otimes v^{\delta_{i, 0}\left(2 d^{\prime}+2\right)-\delta_{i, 0}} \mathbf{K}_{n-1-i}^{\prime \prime} \mathbf{F}_{i}^{\prime \prime}+1 \otimes v^{-\delta_{i, 0}\left(d^{\prime \prime}-1\right)} \mathbf{E}_{n-1-i}^{\prime \prime} . \\
\Delta^{\mathfrak{c} \dagger}\left(\mathbf{k}_{i}\right) & =\mathbf{k}_{i}^{\prime} \otimes \mathbf{K}_{i}^{\prime \prime} \mathbf{K}_{n-1-i}^{\prime \prime-1} .
\end{aligned}
$$

Here the superscripts follow the same convention as in Proposition 5.2.3.

Proof. The third formula on $\Delta^{\mathfrak{c} \dagger}\left(\mathbf{k}_{i}\right)$ is clear.

Suppose that the quadruple $\left(\mathbf{b}^{\prime}, \mathbf{a}^{\prime}, \mathbf{b}^{\prime \prime}, \mathbf{a}^{\prime \prime}\right)$ satisfies the following conditions:

$$
b_{k}^{\prime}=a_{k}^{\prime}-\delta_{\bar{k}, \bar{i}}+\delta_{\bar{k}, \overline{i+1}}+\delta_{\bar{k}, \overline{n-1-i}}-\delta_{\bar{k}, \overline{n-i}}, \quad b_{k}^{\prime \prime}=a_{k}^{\prime \prime}, \quad \forall k, \text { some } i \in[0, r],
$$

where $\bar{k}, \bar{i}$ 's are in $\mathbb{Z} / n \mathbb{Z}$. So we have $s\left(\mathbf{b}^{\prime}, \mathbf{a}^{\prime}, \mathbf{b}^{\prime \prime}, \mathbf{a}^{\prime \prime}\right)=-a_{i}^{\prime \prime}+a_{n-1-i}^{\prime \prime}+\delta_{i, 0} d^{\prime \prime}$ and $u\left(\mathbf{b}^{\prime \prime}, \mathbf{a}^{\prime \prime}\right)=0$.

Suppose that the quadruple $\left(\mathbf{b}^{\prime}, \mathbf{a}^{\prime}, \mathbf{b}^{\prime \prime}, \mathbf{a}^{\prime \prime}\right)$ satisfies the following conditions:

$$
b_{k}^{\prime}=a_{k}^{\prime}, \quad b_{k}^{\prime \prime}=a_{k}^{\prime \prime}-\delta_{\bar{k}, \bar{i}}+\delta_{\bar{k}, \bar{i}+1}, \forall k, \text { some } i \in[0, r] .
$$

Then we have $s\left(\mathbf{b}^{\prime}, \mathbf{a}^{\prime}, \mathbf{b}^{\prime \prime}, \mathbf{a}^{\prime \prime}\right)=a_{i+1}^{\prime}-\delta_{i, 0}\left(2 d^{\prime}+2\right)$, and $u\left(\mathbf{b}^{\prime \prime}, \mathbf{a}^{\prime \prime}\right)=a_{n-1-i}^{\prime \prime}$. 
Suppose that the quadruple $\left(\mathbf{b}^{\prime}, \mathbf{a}^{\prime}, \mathbf{b}^{\prime \prime}, \mathbf{a}^{\prime \prime}\right)$ satisfies the following conditions:

$$
b_{k}^{\prime}=a_{k}^{\prime}, \quad b_{k}^{\prime \prime}=a_{k}^{\prime \prime}+\delta_{\bar{k}, \overline{n-1-i}}-\delta_{\bar{k}, \overline{n-i}}, \quad \forall k, \text { some } i \in[0, r] .
$$

Then we have $s\left(\mathbf{b}^{\prime}, \mathbf{a}^{\prime}, \mathbf{b}^{\prime \prime}, \mathbf{a}^{\prime \prime}\right)=-a_{i}^{\prime}$, and $u\left(\mathbf{b}^{\prime \prime}, \mathbf{a}^{\prime \prime}\right)=-a_{i}^{\prime \prime}+\delta_{i, 0} d^{\prime \prime}$. The above computations lead to the first formula on $\Delta^{\text {c† }}\left(\mathbf{e}_{i}\right)$.

The second formula on $\Delta^{\mathfrak{c} \dagger}\left(\mathbf{f}_{i}\right)$ follows from the following computations. Suppose that the quadruple $\left(\mathbf{b}^{\prime}, \mathbf{a}^{\prime}, \mathbf{b}^{\prime \prime}, \mathbf{a}^{\prime \prime}\right)$ satisfies the following conditions:

$$
b_{k}^{\prime}=a_{k}^{\prime}+\delta_{\bar{k}, \bar{i}}-\delta_{\bar{k}, \overline{i+1}}-\delta_{\bar{k}, \overline{n-1-i}}+\delta_{\bar{k}, \overline{n-i}}, \quad b_{k}^{\prime \prime}=a_{k}^{\prime \prime}, \quad \forall k, \text { some } i \in[0, r] .
$$

Then we have $s\left(\mathbf{b}^{\prime}, \mathbf{a}^{\prime}, \mathbf{b}^{\prime \prime}, \mathbf{a}^{\prime \prime}\right)=a_{i}^{\prime \prime}-a_{n-1-i}^{\prime \prime}-\delta_{i, 0} d^{\prime \prime}$ and $u\left(\mathbf{b}^{\prime \prime}, \mathbf{a}^{\prime \prime}\right)=0$.

Suppose that the quadruple $\left(\mathbf{b}^{\prime}, \mathbf{a}^{\prime}, \mathbf{b}^{\prime \prime}, \mathbf{a}^{\prime \prime}\right)$ satisfies the following conditions:

$$
b_{k}^{\prime}=a_{k}^{\prime}, \quad b_{k}^{\prime \prime}=a_{k}^{\prime \prime}+\delta_{\bar{k}, \bar{i}}-\delta_{\bar{k}, \bar{i}+1}, \forall k, \text { some } i \in[0, r] .
$$

Then we have $s\left(\mathbf{b}^{\prime}, \mathbf{a}^{\prime}, \mathbf{b}^{\prime \prime}, \mathbf{a}^{\prime \prime}\right)=-a_{i+1}^{\prime}+\delta_{i, 0}\left(2 d^{\prime}+2\right)$, and $u\left(\mathbf{b}^{\prime \prime}, \mathbf{a}^{\prime \prime}\right)=-a_{n-1-i}^{\prime \prime}$.

Suppose that the quadruple $\left(\mathbf{b}^{\prime}, \mathbf{a}^{\prime}, \mathbf{b}^{\prime \prime}, \mathbf{a}^{\prime \prime}\right)$ satisfies the following conditions.

$$
b_{k}^{\prime}=a_{k}^{\prime}, \quad b_{k}^{\prime \prime}=a_{k}^{\prime \prime}-\delta_{\bar{k}, \overline{n-1-i}}+\delta_{\bar{k}, \overline{n-i}}, \quad \forall k, \text { some } i \in[0, r] .
$$

Then we have $s\left(\mathbf{b}^{\prime}, \mathbf{a}^{\prime}, \mathbf{b}^{\prime \prime}, \mathbf{a}^{\prime \prime}\right)=a_{i}^{\prime}$, and $u\left(\mathbf{b}^{\prime \prime}, \mathbf{a}^{\prime \prime}\right)=a_{i}^{\prime \prime}-\delta_{i, 0}\left(d^{\prime \prime}-1\right)$.

The proposition is proved.

The above formulas are indeed compatible with the ones in the finite type case for $i \in[1, r]$; cf. [FL15] and Proposition A.3.2, Recall $\xi_{d, i, c}: \mathbf{S}_{n, d} \rightarrow \mathbf{S}_{n, d}$ in affine type $A$ from [FL15]. We generalize it to the affine type $C$ as

$$
\begin{aligned}
\xi_{d, i, c}^{\mathfrak{c}}: \mathbf{S}_{n, d}^{\mathfrak{c}} \longrightarrow \mathbf{S}_{n, d}^{\mathfrak{c}}, & \text { for } i \in[0, r], c \in \mathbb{Z}, \\
\xi_{d, i, c}^{\mathfrak{c}}([A])=v^{c \varepsilon_{i}(A)}[A], & \text { for } A \in \Xi_{n, d},
\end{aligned}
$$

where

$$
\varepsilon_{i}(A)=\sum_{r \leqslant i<s} a_{r s}-\sum_{r>i \geqslant s} a_{r s}
$$

In particular, we have

$$
\xi_{d, i, c}^{\mathfrak{c}}\left(\mathbf{e}_{j}\right)=v^{-c \delta_{i, j}} \mathbf{e}_{j}, \quad \xi_{d, i, c}^{\mathfrak{c}}\left(\mathbf{f}_{j}\right)=v^{c \delta_{i, j}} \mathbf{f}_{j}, \quad \xi_{d, i, c}^{\mathfrak{c}}\left(\mathbf{k}_{j}\right)=\mathbf{k}_{j}
$$

We define the algebra homomorphism (which is a refined comultiplication from the raw multiplication $\widetilde{\Delta}^{\mathfrak{c}}$ )

$$
\Delta^{\mathfrak{c}} \equiv \Delta_{d^{\prime}, d^{\prime \prime}}^{\mathfrak{c}}=\left(\xi_{d^{\prime}, 0, d^{\prime \prime}}^{\mathfrak{c}} \otimes \xi_{d^{\prime \prime}, 0,-\left(2 d^{\prime}+2\right)} \xi_{d^{\prime \prime}, n-1,-\left(d^{\prime \prime}-1\right)}\right) \circ \Delta^{\mathfrak{c} \dagger}: \mathbf{S}_{n, d}^{\mathfrak{c}} \longrightarrow \mathbf{S}_{n, d^{\prime}}^{\mathfrak{c}} \otimes \mathbf{S}_{n, d^{\prime \prime}}
$$

Proposition 5.3.2. For all $i \in[0, r]$ and $A \in \Xi_{n, d}$, we have $\xi_{d, i, c}^{\mathfrak{c}}\left(\{A\}_{d}\right)=v^{c \varepsilon_{i}(A)}\{A\}_{d}$. 
Proof. By the definition (5.3.5) and using $a_{r s}=a_{-r,-s}$, we have

$$
\begin{aligned}
\varepsilon_{i}(A) & =\left(\sum_{r \leqslant i<s}-\sum_{r<-i \leqslant s}\right) a_{r s}=\left(\sum_{\substack{i \leqslant r \leqslant i \\
i<s}}-\sum_{\substack{-i \leqslant s \leqslant i \\
r<-i}}\right) a_{r s} \\
& =\frac{1}{2}\left(\sum_{\substack{i \leqslant r \leqslant i \\
i<s}}+\sum_{\substack{-i \leqslant r \leqslant i \\
s<-i}}-\sum_{\substack{i \leqslant s \leqslant i \\
r<-i}}-\sum_{\substack{-i \leqslant s \leqslant i \\
r>i}}\right) a_{r s} \\
& =\frac{1}{2}\left(\sum_{-i \leqslant r \leqslant i}-\sum_{-i \leqslant s \leqslant i}\right) a_{r s}=\frac{1}{2} \sum_{-i \leqslant s \leqslant i} \operatorname{ro}(A)_{s}-\operatorname{co}(A)_{s} .
\end{aligned}
$$

Now if the polynomial $P_{A, B}$ in (4.2.12) is not zero, then $\operatorname{ro}(B)=\operatorname{ro}(A)$ and $\operatorname{co}(B)=\operatorname{co}(A)$, and hence $\varepsilon_{i}(A)=\varepsilon_{i}(B)$. Therefore, we have

$$
\begin{aligned}
\xi_{d, i, c}^{\mathfrak{c}}\left(\{A\}_{d}\right) & =\xi_{d, i, c}^{\mathfrak{c}}\left(\sum_{B \leqslant A} P_{A, B}[B]\right)=\sum_{B \leqslant A} P_{A, B} v^{c \varepsilon_{i}(B)}[B] \\
& =v^{c \varepsilon_{i}(A)} \sum_{B \leqslant A} P_{A, B}[B]=v^{c \varepsilon_{i}(A)}\{A\}_{d} .
\end{aligned}
$$

The proposition is proved.

Let $\mathbf{a}, \mathbf{b} \in \Lambda_{n, d}^{\mathfrak{c}}$. Fix $L \in \mathcal{X}_{n, d}^{\mathfrak{c}}(\mathbf{b})$ (which was defined in (15.3.2) ), and let $P_{\mathbf{b}}=\operatorname{Stab}_{\operatorname{Sp}_{F}(2 d)}(L)$. We have a natural embedding

$$
\iota_{\mathbf{b}, \mathbf{a}}: \mathcal{X}_{n, d}^{\mathfrak{c}}(\mathbf{a}) \longrightarrow \mathcal{X}_{n, d}^{\mathfrak{c}}(\mathbf{b}) \times \mathcal{X}_{n, d}^{\mathfrak{c}}(\mathbf{a}), \quad L^{\prime} \mapsto\left(L, L^{\prime}\right)
$$

It is well known that $\iota_{\mathbf{b}, \mathbf{a}}$ induces the following isomorphism of $\mathcal{A}$-modules:

$$
\iota_{\mathbf{b}, \mathbf{a}}^{*}: \mathcal{A}_{\operatorname{Sp}_{F}(2 d)}\left(\mathcal{X}_{n, d}^{\mathfrak{c}}(\mathbf{b}) \times \mathcal{X}_{n, d}^{\mathfrak{c}}(\mathbf{a})\right) \longrightarrow \mathcal{A}_{P_{\mathbf{b}}}\left(\mathcal{X}_{n, d}^{\mathfrak{c}}(\mathbf{a})\right) .
$$

Let

$$
\mathcal{X}_{\mathbf{a}, \mathbf{a}^{\prime}, \mathbf{a}^{\prime \prime}}^{\mathfrak{c}}=\left\{L \in \mathcal{X}_{n, d}^{\mathfrak{c}}(\mathbf{a}) \mid \pi^{\natural}(L) \in \mathcal{X}_{n, d^{\prime}}^{\mathfrak{c}}\left(\mathbf{a}^{\prime}\right), \pi^{\prime \prime}(L) \in \mathcal{X}_{n, d^{\prime \prime}}\left(\mathbf{a}^{\prime \prime}\right)\right\} .
$$

Then we have the following diagram

$$
\mathcal{X}_{n, d}^{\mathfrak{c}}(\mathbf{a}) \longleftarrow \stackrel{\iota}{\longleftarrow} \mathcal{X}_{\mathbf{a}, \mathbf{a}^{\prime}, \mathbf{a}^{\prime \prime}}^{\mathfrak{c}} \longrightarrow \mathcal{X}_{n, d^{\prime}}^{\mathfrak{c}}\left(\mathbf{a}^{\prime}\right) \times \mathcal{X}_{n, d^{\prime \prime}}\left(\mathbf{a}^{\prime \prime}\right),
$$

where $\iota$ is the imbedding and $\pi(L)=\left(\pi^{\natural}(L), \pi^{\prime \prime}(L)\right)$. By identifying $\mathcal{A}_{P_{\mathbf{b}^{\prime}} \times P_{\mathbf{b}^{\prime \prime}}}\left(\mathcal{X}_{n, d^{\prime}}^{\mathfrak{c}}\left(\mathbf{a}^{\prime}\right) \times\right.$ $\left.\mathcal{X}_{n, d^{\prime \prime}}\left(\mathbf{a}^{\prime \prime}\right)\right)=\mathcal{A}_{P_{\mathbf{b}^{\prime}}}\left(\mathcal{X}_{n, d^{\prime}}^{\mathfrak{c}}\left(\mathbf{a}^{\prime}\right)\right) \times \mathcal{A}_{P_{\mathbf{b}^{\prime \prime}}}\left(\mathcal{X}_{n, d^{\prime \prime}}\left(\mathbf{a}^{\prime \prime}\right)\right)$, we have the following linear map

$$
\pi ! \iota^{*}: \mathcal{A}_{P_{\mathbf{b}}}\left(\mathcal{X}_{n, d}^{\mathfrak{c}}(\mathbf{a})\right) \longrightarrow \mathcal{A}_{P_{\mathbf{b}^{\prime}}}\left(\mathcal{X}_{n, d^{\prime}}^{\mathfrak{c}}\left(\mathbf{a}^{\prime}\right)\right) \times \mathcal{A}_{P_{\mathbf{b}^{\prime \prime}}}\left(\mathcal{X}_{n, d^{\prime \prime}}\left(\mathbf{a}^{\prime \prime}\right)\right)
$$

By a similar argument as for [FL15, Lemma 1.3.5], the following diagram commutes:

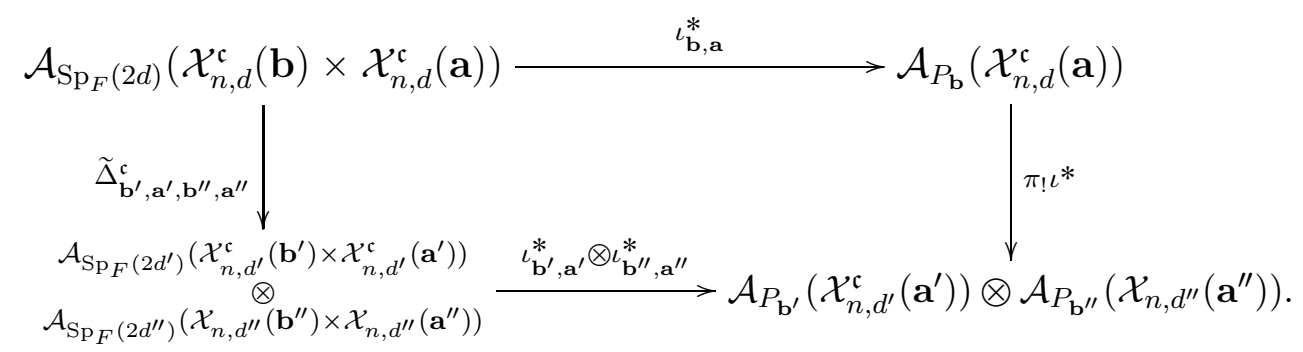

Recall $\Delta^{\mathfrak{c}}: \mathbf{S}_{n, d}^{\mathfrak{c}} \longrightarrow \mathbf{S}_{n, d^{\prime}}^{\mathfrak{c}} \otimes \mathbf{S}_{n, d^{\prime \prime}}$ from (5.3.6). 
Proposition 5.3.3. For $A \in \Xi_{n, d}$, write

$$
\Delta^{\mathfrak{c}}\left(\{A\}_{d}\right)=\sum_{A^{\prime} \in \Xi_{n, d^{\prime}}, A^{\prime \prime} \in \Theta_{n, d^{\prime \prime}}} h_{A}^{A^{\prime}, A^{\prime \prime}}\left\{A^{\prime}\right\}_{d^{\prime}} \otimes{ }^{\mathfrak{a}}\left\{A^{\prime \prime}\right\}_{d^{\prime \prime}}
$$

Then $h_{A}^{A^{\prime}, A^{\prime \prime}} \in \mathbb{N}\left[v, v^{-1}\right]$ for all $A, A^{\prime}$ and $A^{\prime \prime}$.

Proof. By Proposition 5.3.2, the proof is reduced to showing the same type of positivity with respect to $\widetilde{\Delta}^{\mathfrak{c}}$. By an argument similar to [FL15, Section 2.4] and (5.3.7), the positivity for $\widetilde{\Delta}^{\mathfrak{c}}$ follows from [Br03, Theorem 8]. The proposition is proved.

Now let us study the restriction of $\Delta^{\mathfrak{c}}$ to $\mathbf{U}_{n, d^{\prime}}^{\mathfrak{c}}$.

Proposition 5.3.4. Let $d=d^{\prime}+d^{\prime \prime}$. We have a homomorphism $\Delta^{\mathfrak{c}}: \mathbf{U}_{n, d}^{\mathfrak{c}} \longrightarrow \mathbf{U}_{n, d^{\prime}}^{\mathfrak{c}} \otimes \mathbf{U}_{n, d^{\prime \prime}}$. More precisely, for all $i \in[0, r]$, we have

$$
\begin{aligned}
\Delta^{\mathfrak{c}}\left(\mathbf{e}_{i}\right) & =\mathbf{e}_{i}^{\prime} \otimes \mathbf{K}_{i}^{\prime \prime}+1 \otimes \mathbf{E}_{i}^{\prime \prime}+\mathbf{k}_{i}^{\prime} \otimes v^{\delta_{i, 0}} \mathbf{F}_{n-1-i}^{\prime \prime} \mathbf{K}_{i}^{\prime \prime} \\
\Delta^{\mathfrak{c}}\left(\mathbf{f}_{i}\right) & =\mathbf{f}_{i}^{\prime} \otimes \mathbf{K}_{n-1-i}^{\prime \prime}+\mathbf{k}_{i}^{\prime-1} \otimes v^{-\delta_{i, 0}} \mathbf{K}_{n-1-i}^{\prime \prime} \mathbf{F}_{i}^{\prime \prime}+1 \otimes \mathbf{E}_{n-1-i}^{\prime \prime}, \\
\Delta^{\mathfrak{c}}\left(\mathbf{k}_{i}\right) & =\mathbf{k}_{i}^{\prime} \otimes \mathbf{K}_{i}^{\prime \prime} \mathbf{K}_{n-1-i}^{\prime \prime-1} .
\end{aligned}
$$

Recall the comultiplication $\Delta$ in the affine type $A$ from [FL15] (see also [Lu00] for a related construction). This is an algebra homomorphism

$$
\Delta: \mathbf{S}_{n, d} \longrightarrow \mathbf{S}_{n, d^{\prime}} \otimes \mathbf{S}_{n, d^{\prime \prime}}
$$

defined by

$$
\begin{aligned}
\Delta\left(\mathbf{E}_{i}\right) & =\mathbf{E}_{i}^{\prime} \otimes \mathbf{K}_{i}^{\prime \prime}+1 \otimes \mathbf{E}_{i}^{\prime \prime}, \\
\Delta\left(\mathbf{F}_{i}\right) & =\mathbf{F}_{i}^{\prime} \otimes 1+\mathbf{K}_{i}^{\prime-1} \otimes \mathbf{F}_{i}^{\prime \prime}, \\
\Delta\left(\mathbf{K}_{i}\right) & =\mathbf{K}_{i}^{\prime} \otimes \mathbf{K}_{i}^{\prime \prime}, \quad \forall 0 \leqslant i \leqslant n-1 .
\end{aligned}
$$

Here the superscripts follow the same convention in Proposition 5.2.3.

Proposition 5.3.5. The following coassociativity holds on $\mathbf{U}_{n, d}^{\mathfrak{c}}$ :

$$
(1 \otimes \Delta) \Delta^{\mathfrak{c}}=\left(\Delta^{\mathfrak{c}} \otimes 1\right) \Delta^{\mathfrak{c}}
$$

Proof. Beyond type $A$ or finite type $B / C$ we only need to check the desired identity when acting on $\mathbf{e}_{0}, \mathbf{f}_{0}$ and $\mathbf{k}_{0}^{ \pm}$. This can be verified directly.

Now setting $d^{\prime}=0$, we have $\mathbf{e}_{i}^{\prime}=0, \mathbf{f}_{i}^{\prime}=0, \mathbf{k}_{i}^{\prime}=v^{-\delta_{i, 0}+\delta_{i, r}}$ in $\mathbf{S}_{n, 0}^{\mathfrak{c}}$, and $\Delta^{\mathfrak{c}}$ becomes the following algebra homomorphism

$$
\begin{aligned}
\jmath_{n, d} & : \mathbf{S}_{n, d}^{\mathfrak{c}} \longrightarrow \mathbf{S}_{n, d} \\
\jmath_{n, d}\left(\mathbf{e}_{i}\right) & =\mathbf{E}_{i}+v^{-\delta_{i, 0}} \mathbf{K}_{i} \mathbf{F}_{n-1-i}, \\
\jmath_{n, d}\left(\mathbf{f}_{i}\right) & =\mathbf{E}_{n-1-i}+v^{\delta_{i, 0}} \mathbf{F}_{i} \mathbf{K}_{n-1-i}, \\
\jmath_{n, d}\left(\mathbf{k}_{i}\right) & =v^{-\delta_{i, 0}+\delta_{i, r}} \mathbf{K}_{i} \mathbf{K}_{n-1-i}^{-1}, \quad \forall i \in[0, r] .
\end{aligned}
$$

It follows by restriction that we have also a homomorphism $\jmath_{n, d}: \mathbf{U}_{n, d}^{\mathfrak{c}} \rightarrow \mathbf{U}_{n, d}$. Thanks to Propositions 2.2 .3 and 5.4.1, the same argument as in finite type $B / C$ [FL15] gives us the following. 
Proposition 5.3.6. The homomorphism $\jmath_{n, d}: \mathbf{S}_{n, d}^{\mathfrak{c}} \rightarrow \mathbf{S}_{n, d}\left(\right.$ and $\jmath_{n, d}: \mathbf{U}_{n, d}^{\mathfrak{c}} \rightarrow \mathbf{U}_{n, d}$ ) is injective.

Proposition 5.3 .3 in our setting of $d^{\prime}=0$ gives us the following.

Proposition 5.3.7. The map $\jmath_{n, d}$ sends a canonical basis element in $\mathbf{S}_{n, d}^{\mathfrak{c}}$ to a sum of canonical basis elements of $\mathbf{S}_{n, d}$ with coefficients in $\mathbb{N}\left[v, v^{-1}\right]$.

\subsection{Monomial and CAnONical Bases of $\mathbf{U}_{n, d}^{\mathfrak{c}}$}

Recall $\Xi_{n, d}$ from (4.2.1) and the notion of aperiodic matrices from (2.2.6). We denote

$$
\Xi_{n, d}^{a p}=\left\{A \in \Xi_{n, d} \mid A \text { is aperiodic }\right\} .
$$

A product of standard basis elements $\left[G_{1}\right] *\left[G_{2}\right] * \cdots *\left[G_{m}\right]$ in $\mathbf{S}_{n, d}^{\mathfrak{c}}$ is called an aperiodic monomial if for each $i, G_{i}-R E_{\theta}^{j, j+1}$ is diagonal for some $R \in \mathbb{N}$ and $j \in \mathbb{Z}$. The following aperiodic monomial is an analogue of $\zeta_{A}^{\mathfrak{a}}$ for $\mathbf{U}_{n, d}$ (see Proposition [2.2.3).

Proposition 5.4.1. For any $A \in \Xi_{n, d}^{a p}$, there exists an aperiodic monomial $\zeta_{A} \in \mathbf{U}_{n, d}^{\mathfrak{c}}$ such that

$$
\zeta_{A}=[A]+\text { lower terms. }
$$

Proof. With the help of Lemma 4.4.2, the proof is the same as that for Proposition 2.2.3.

While the aperiodic monomial $\zeta_{A}$ with (5.4.2) is not unique, we shall fix one for each $A$.

The following type $C$ aperiodicity follows from two kinds of positivity properties and the highly nontrivial affine type $A$ aperiodicity in [Lu99, Proposition 6.5].

Proposition 5.4.2. Let $M$ be an aperiodic monomial in $\mathbf{S}_{n, d}^{\mathfrak{c}}$. Suppose that $M=\sum c_{A}\{A\}_{d}$ where $c_{A} \in \mathbb{Z}\left[v, v^{-1}\right]$. If $c_{A} \neq 0$, then $A$ must be aperiodic.

Proof. Recall the canonical basis elements in $\mathbf{S}_{n, d}$ (and in $\mathbf{U}_{n, d}$ ) are denoted by $\mathfrak{a}\{A\}_{d}$, and note that $\mathbf{U}_{n, d}$ is generated by $\mathbf{E}_{i}, \mathbf{F}_{i}$ and $\mathbf{K}_{i}^{ \pm 1}$ for all $1 \leqslant i \leqslant n$. By (5.3.9), we have $\jmath_{n, d}(\mathrm{M}) \in \mathbf{U}_{n, d}$. By [Lu99, Proposition 6.5], we see that

$$
\jmath_{n, d}(\mathrm{M})=\sum_{B \text { aperiodic }} g_{B}{ }^{\mathfrak{a}}\{B\}_{d}, \quad \text { where } g_{B} \in \mathbb{N}\left[v, v^{-1}\right] .
$$

For $A=\left(a_{i j}\right) \in \Xi_{n, d}$, we set

$$
\Xi_{n, d}(A)=\left\{B=\left(b_{i j}\right) \in \Theta_{n, d} \mid b_{i j}=0, \forall i<j, b_{i j}=a_{i j}, \forall i>j, \operatorname{co}(B) \models \operatorname{co}(A)\right\},
$$

where the notation ' $\mathbf{b} \models \mathbf{a}$ ' stands for $b_{i}+b_{-i}+\delta_{i, n}+\delta_{i, r+1}=a_{i}$ for all $1 \leqslant i \leqslant n$. In particular, if $A$ is aperiodic, so is any matrix in $\Xi_{n, d}(A)$. Since $\zeta_{A}=\{A\}_{d}+$ lower terms by Proposition 5.4.1, it implies that

$$
\jmath_{n, d}\left(\{A\}_{d}\right)=\sum_{A^{-} \in \Xi_{n, d}(A)}{ }^{\mathfrak{a}}\left\{A^{-}\right\}_{d}+\sum_{A^{-} \in \Xi_{n, d}(A)} \sum_{B<A^{-}} h_{A^{-}, B}{ }^{\mathfrak{a}}\{B\}_{d}+R_{A}, \quad h_{A^{-}, B} \in \mathbb{N}\left[v, v^{-1}\right],
$$

where $R_{A}$ is a linear combination of ${ }^{\mathfrak{a}}\{B\}_{d}$ over $\mathbb{N}\left[v, v^{-1}\right]$ for those $B$ not lower triangular. Indeed, this can be proved by induction on the length of the monomial $\zeta_{A}$ and utilizing the fact that the action of the Chevalley generators $\mathbf{F}_{i}^{(a)}$ on a standard basis element of a lower triangular matrix $A$ gives rise to a linear combination of standard basis element of 
either lower triangular matrices $A^{\prime}<A$ or non-lower-triangular matrices. The latter is an observation from the multiplication formula for the Chevalley generator $\mathbf{F}_{i}^{(a)}$ in $(2.2 .10)$. So

$$
\begin{aligned}
\jmath_{n, d}(\mathrm{M}) & =\jmath_{n, d}\left(\sum_{A} c_{A}\{A\}_{d}\right) \\
& =\sum_{A} \sum_{A^{-} \in \Xi_{n, d}(A)} c_{A}{ }^{\mathfrak{a}}\left\{A^{-}\right\}_{d}+\sum_{A} \sum_{A^{-} \in \Xi_{n, d}(A)} \sum_{B<A^{-}} c_{A} h_{A^{-}, B}{ }^{\mathfrak{a}}\{B\}_{d}+\sum_{A} c_{A} R_{A} .
\end{aligned}
$$

Observe also that $c_{A} \in \mathbb{N}\left[v, v^{-1}\right]$ due to the geometric interpretation of $\mathrm{M}$. This implies that the coefficient of ${ }^{\mathfrak{a}}\left\{A^{-}\right\}_{d}$ in (5.4.5) is $c_{A}$ plus some terms in $\mathbb{N}\left[v, v^{-1}\right]$ since $h_{A^{-}, B} \in \mathbb{N}\left[v, v^{-1}\right]$, hence nonzero. By comparing (5.4.3) and (5.4.5), we see that $A^{-}$are all aperiodic. Therefore $A$ is aperiodic. The proposition is proved.

Theorem 5.4.3. The set $\left\{\{A\}_{d} \mid A \in \Xi_{n, d}^{a p}\right\}$ forms a basis (called the canonical basis) of $\mathbf{U}_{n, d}^{\mathfrak{c}}$. Also, the set $\left\{\zeta_{A} \mid A \in \Xi_{n, d}^{a p}\right\}$ forms a basis (called a monomial basis) of $\mathbf{U}_{n, d}^{\mathfrak{c}}$.

Proof. For $A \in \Xi_{n, d}^{a p}$, we have $\zeta_{A}=[A]+$ lower terms by Proposition 5.4.1, and so $\zeta_{A} \in\{A\}_{d}+$ $\sum_{A^{\prime}<A} \mathcal{A}\left\{A^{\prime}\right\}_{d}$; this sum can be additionally restricted to $A^{\prime} \in \Xi_{n, d}^{a p}$ by Proposition 5.4.2. Hence by an induction on $A$ by the partial ordering, we conclude that $\{A\}_{d} \in \mathbf{U}_{n, d}^{\mathfrak{c}}$. Since $\left\{\{A\}_{d} \mid A \in \Xi_{n, d}^{a p}\right\}$ is clearly linearly independent and it forms a spanning set of $\mathbf{U}_{n, d}^{\mathfrak{c}}$ by Proposition 5.4.2, it is a basis of $\mathbf{U}_{n, d}^{\mathfrak{c}}$.

Since the transition matrix from $\left\{\zeta_{A} \mid A \in \Xi_{n, d}^{a p}\right\}$ to the canonical basis is uni-triangular, $\left\{\zeta_{A} \mid A \in \Xi_{n, d}^{a p}\right\}$ forms a basis as well.

The next proposition follows from Propositions 5.3.3, 5.3.4 and Theorem 5.4.3.

Proposition 5.4.4. For $B \in \Xi_{n, d}^{a p}$, write $\Delta^{\mathfrak{c}}\left(\{B\}_{d}\right)=\sum_{C \in \Xi_{n, d^{\prime}}^{a p}, A \in \Theta_{n, d^{\prime \prime}}^{a p}} \hat{m}_{B}^{C, A}\{C\}_{d^{\prime}} \otimes \mathfrak{a}\{A\}_{d^{\prime \prime}}$. Then we have $\hat{m}_{B}^{C, A} \in \mathbb{N}\left[v, v^{-1}\right]$.

We also have the following corollary of Proposition 5.3.7 and Theorem 5.4.3.

Corollary 5.4.5. The image of $\jmath_{n, d}$ of a canonical basis element in $\mathbf{U}_{n, d}^{\mathfrak{c}}$ is a sum of canonical basis elements of $\mathbf{U}_{n, d}$ with coefficients in $\mathbb{N}\left[v, v^{-1}\right]$. 
Part 2. Lusztig algebras and coideal subalgebras of $\mathbf{U}\left(\widehat{\mathfrak{s l}}_{n}\right)$ 


\section{CHAPTER 6}

\section{Realization of the idempotented coideal subalgebra $\dot{\mathbf{U}}_{n}^{\mathfrak{c}}$ of $\mathbf{U}\left(\hat{\mathfrak{s l}}_{n}\right)$}

In this chapter we introduce the transfer maps $\phi_{d, d-n}^{\mathfrak{c}}$ on Schur algebras $\mathbf{S}_{n, d}^{\mathfrak{c}}$ and Lusztig algebras $\mathbf{U}_{n, d}^{\mathfrak{c}}$. We then construct algebras $\mathbf{U}_{n}^{\mathfrak{c}}\left(\right.$ or $\dot{\mathbf{U}}_{n}^{\mathfrak{c}}$ ) from the projective system of algebras $\left\{\left(\mathbf{U}_{n, d}^{\mathfrak{c}}, \phi_{d, d-n}^{\mathfrak{c}}\right)\right\}_{d \geqslant 0}$. We show that $\mathbf{U}_{n}^{\mathfrak{c}}\left(\right.$ or $\left.\dot{\mathbf{U}}_{n}^{\mathfrak{c}}\right)$ is isomorphic to an (idempotented) coideal subalgebra of $\mathbf{U}\left(\widehat{\mathfrak{s l}}_{n}\right)$, and $\left(\mathbf{U}\left(\widehat{\mathfrak{s l}}_{n}\right), \mathbf{U}_{n}^{\mathfrak{c}}\right)$ forms an affine quantum symmetric pair. The canonical basis of $\dot{\mathbf{U}}_{n}^{\mathfrak{c}}$ is established and shown to admit positivity with respect to multiplication, comultiplication, and a bilinear pairing.

\subsection{The COIDEAL SUBAlgebra $\mathbf{U}_{n}^{\mathfrak{c}}$ oF $\mathbf{U}_{n}$}

Recall [Lu00] there exists a homomorphism $\chi_{n}: \mathbf{S}_{n, n} \rightarrow \mathbb{Q}(v)$ such that

$$
\chi_{n}\left(\mathbf{E}_{i}\right)=\chi_{n}\left(\mathbf{F}_{i}\right)=0, \chi_{n}\left(\mathbf{H}_{i}\right)=v .
$$

Following Lusztig [Lu00], we introduce the transfer map of affine type $C$,

$$
\phi_{d, d-n}^{\mathfrak{c}}: \mathbf{S}_{n, d}^{\mathfrak{c}} \rightarrow \mathbf{S}_{n, d-n}^{\mathfrak{c}},
$$

which is by definition the composition of the following homomorphisms (for $d \geqslant n$ )

$$
\phi_{d, d-n}^{\mathfrak{c}}: \mathbf{S}_{n, d}^{\mathfrak{c}} \stackrel{\widetilde{\Delta}^{\mathfrak{c}}}{\longrightarrow} \mathbf{S}_{n, d-n}^{\mathfrak{c}} \otimes \mathbf{S}_{n, n} \stackrel{1 \otimes \chi_{n}}{\longrightarrow} \mathbf{S}_{n, d-n}^{\mathfrak{c}}
$$

The following can be proved similarly to [Lu00] in affine type $A$ and [FL15] in type $B / C$.

Proposition 6.1.1. For $i \in[0, r]$, we have $\phi_{d, d-n}^{\mathfrak{c}}\left(\mathbf{e}_{i}\right)=\mathbf{e}_{i}^{\prime}, \phi_{d, d-n}^{\mathfrak{c}}\left(\mathbf{f}_{i}\right)=\mathbf{f}_{i}^{\prime}, \phi_{d, d-n}^{\mathfrak{c}}\left(\mathbf{k}_{i}\right)=\mathbf{k}_{i}^{\prime}$.

Now we consider the projective system $\left\{\left(\mathbf{U}_{n, d}^{\mathfrak{c}}, \phi_{d, d-n}^{\mathfrak{c}}\right)\right\}_{d \geqslant 0}$ and its projective limit:

$$
\mathbf{U}_{n, \infty}^{\mathfrak{c}}:=\lim _{d} \mathbf{U}_{n, d}^{\mathfrak{c}}=\left\{x \equiv\left(x_{d}\right)_{d \in \mathbb{N}} \in \prod_{d \in \mathbb{N}} \mathbf{U}_{n, d}^{\mathfrak{c}} \mid \phi_{d, d-n}^{\mathfrak{c}}\left(x_{d}\right)=x_{d-n} \quad \forall d\right\} .
$$

Denote by $\phi_{d}^{\mathfrak{c}}: \mathbf{U}_{n, \infty}^{\mathfrak{c}} \rightarrow \mathbf{U}_{n, d}^{\mathfrak{c}}$ the natural projection. The bar involution on $\mathbf{U}_{n, d}^{\mathfrak{c}}$ induces a bar involution ${ }^{-}: \mathbf{U}_{n, \infty}^{\mathfrak{c}} \rightarrow \mathbf{U}_{n, \infty}^{\mathfrak{c}}$, since it commutes with the transfer map (6.1.1). Similarly, we have an integral version: $\mathbf{U}_{n, \infty ; \mathcal{A}}^{\mathfrak{c}}=\lim _{d} \mathbf{U}_{n, d ; \mathcal{A}}^{\mathfrak{c}}$. Since $\mathbb{Q}(v) \otimes_{\mathcal{A}} \mathbf{U}_{n, d ; \mathcal{A}}^{\mathfrak{c}}=\mathbf{U}_{n, d}^{\mathfrak{c}}$ for all $d$, we have $\mathbb{Q}(v) \otimes_{\mathcal{A}} \mathbf{U}_{n, \infty ; \mathcal{A}}^{\mathfrak{c}}=\mathbf{U}_{n, \infty}^{\mathfrak{c}}$.

Recall from Section 2.3 the counterparts of the above constructions in the affine type $A$ setting, where we drop the superscript $\mathfrak{c}$. We have the following commutative diagram

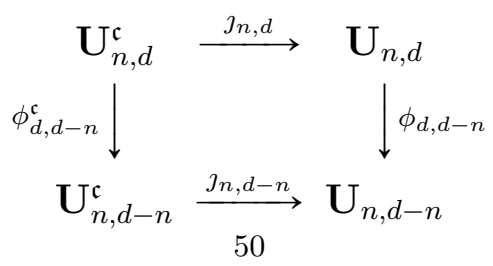


That is, $\phi_{d, d-n} \circ \jmath_{n, d}=\jmath_{n, d-n} \circ \phi_{d, d-n}^{\mathfrak{c}}$. Thus by the universality of $\mathbf{U}_{n, \infty}$, we have a unique algebra homomorphism

such that $\phi_{d} \circ \jmath_{n}=\jmath_{n, d} \circ \phi_{d}^{\mathfrak{c}}$.

$$
\jmath_{n}: \mathbf{U}_{n, \infty}^{\mathfrak{c}} \longrightarrow \mathbf{U}_{n, \infty}
$$

We define elements $\mathbf{e}_{i}, \mathbf{f}_{i}$ and $\mathbf{k}_{i}^{ \pm 1}$ for all $0 \leqslant i \leqslant r$ in $\mathbf{U}_{n, \infty}^{\mathfrak{c}}$ by

$$
\left(\mathbf{e}_{i}\right)_{d}=\mathbf{e}_{i, d},\left(\mathbf{f}_{i}\right)_{d}=\mathbf{f}_{i, d},\left(\mathbf{k}_{i}^{ \pm 1}\right)_{d}=\mathbf{k}_{i, d}^{ \pm 1}, \quad \forall d \in \mathbb{N},
$$

where the $d$ in the subscript of $\mathbf{e}_{i, d}$ etc. indicates $\mathbf{e}_{i, d}$ is a copy of the Chevalley generator $\mathbf{e}_{i}$ in $\mathbf{U}_{n, d}^{\mathfrak{c}}$. Let $\mathbf{U}_{n}^{\mathfrak{c}}$ be the subalgebra of $\mathbf{U}_{n, \infty}^{\mathfrak{c}}$ generated by (the Chevalley generators) $\mathbf{e}_{i}, \mathbf{f}_{i}$ and $\mathbf{k}_{i}^{ \pm 1}$ for all $0 \leqslant i \leqslant r$. Since $\jmath_{n, d}$ is injective for all $d$, so is $\jmath_{n}: \mathbf{U}_{n, \infty}^{\mathfrak{c}} \rightarrow \mathbf{U}_{n, \infty}$. It follows by (5.3.9) that the image of $\mathbf{U}_{n}^{\mathfrak{c}}$ under $\jmath_{n}$ lies in $\mathbf{U}_{n}$. Summarizing, we have obtained the following.

Proposition 6.1.2. There is a unique algebra imbedding $\jmath_{n}: \mathbf{U}_{n}^{\mathfrak{c}} \rightarrow \mathbf{U}_{n}$ such that

$$
\begin{aligned}
\jmath_{n}\left(\mathbf{e}_{i}\right) & =\mathbf{E}_{i}+v^{-\delta_{i, 0}} \mathbf{K}_{i} \mathbf{F}_{n-1-i}, \\
\jmath_{n}\left(\mathbf{f}_{i}\right) & =\mathbf{E}_{n-1-i}+v^{\delta_{i, 0}} \mathbf{F}_{i} \mathbf{K}_{n-1-i}, \\
\jmath_{n}\left(\mathbf{k}_{i}\right) & =v^{-\delta_{i, 0}+\delta_{i, r}} \mathbf{K}_{i} \mathbf{K}_{n-1-i}^{-1}, \quad \forall i \in[0, r] .
\end{aligned}
$$

Recall from Proposition 2.3.2 that $\mathbf{U}_{n} \cong \mathbf{U}\left(\widehat{\mathfrak{s l}}_{n}\right)$. At the $v=1$ limit, the images of the generators under $\jmath_{n}$ are in the fixed point subalgebra by an involution $\theta^{\jmath \jmath}$ of $\widehat{\mathfrak{s l}}_{n}$ (which switches E's and F's); for an illustration of $\theta^{\jmath \jmath}$ see Figure 1.

Recall $\Delta^{\mathfrak{c}}$ from (5.3.6). We have the following commutative diagram

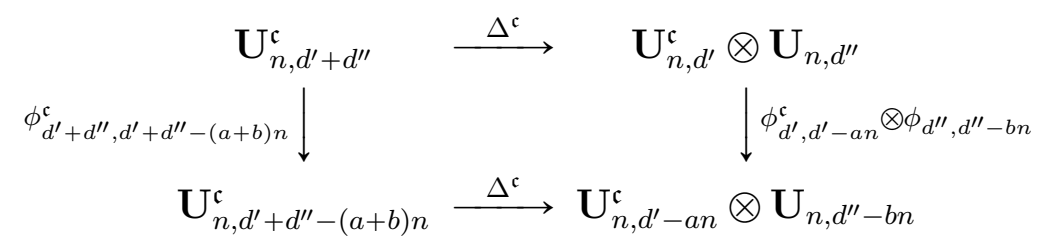

for any $a, b \in \mathbb{N}$. By universality, these $\Delta^{\mathfrak{c}}\left(\right.$ for $\left.d^{\prime}, d^{\prime \prime}, n\right)$ induce an algebra homomorphism

$$
\Delta^{\mathfrak{c}}: \mathbf{U}_{n, \infty}^{\mathfrak{c}} \longrightarrow \mathbf{U}_{n, \infty}^{\mathfrak{c}} \otimes \mathbf{U}_{n, \infty}
$$

Moreover, the image of $\mathbf{U}_{n}^{\mathfrak{c}}$ under $\Delta_{n}^{\mathfrak{c}}$ is contained in $\mathbf{U}_{n}^{\mathfrak{c}} \otimes \mathbf{U}_{n}$ by Proposition 5.3.4. Summarizing, we have the following.

Proposition 6.1.3. There is a unique algebra homomorphism $\Delta^{\mathfrak{c}}: \mathbf{U}_{n}^{\mathfrak{c}} \longrightarrow \mathbf{U}_{n}^{\mathfrak{c}} \otimes \mathbf{U}_{n}$ such that, for all $i \in[0, r]$,

$$
\begin{aligned}
\Delta^{\mathfrak{c}}\left(\mathbf{e}_{i}\right) & =\mathbf{e}_{i} \otimes \mathbf{K}_{i}+1 \otimes \mathbf{E}_{i}+\mathbf{k}_{i} \otimes v^{\delta_{i, 0}} \mathbf{F}_{n-1-i} \mathbf{K}_{i} . \\
\Delta^{\mathfrak{c}}\left(\mathbf{f}_{i}\right) & =\mathbf{f}_{i} \otimes \mathbf{K}_{n-1-i}+\mathbf{k}_{i}^{-1} \otimes v^{-\delta_{i, 0}} \mathbf{K}_{n-1-i} \mathbf{F}_{i}+1 \otimes \mathbf{E}_{n-1-i} . \\
\Delta^{\mathfrak{c}}\left(\mathbf{k}_{i}\right) & =\mathbf{k}_{i} \otimes \mathbf{K}_{i} \mathbf{K}_{n-1-i}^{-1} .
\end{aligned}
$$

This algebra homomorphism is coassociative by Proposition 5.3.5 in the sense that

$$
(1 \otimes \Delta) \Delta^{\mathfrak{c}}=\left(\Delta^{\mathfrak{c}} \otimes 1\right) \Delta^{\mathfrak{c}}
$$

As a degenerate case for (6.1.6), we also have

$$
\Delta \circ \jmath_{n}=\left(\jmath_{n} \otimes 1\right) \circ \Delta^{\mathfrak{c}} .
$$


Summarizing the results from Propositions 6.1.2 and 6.1.3, we have proved the following.

Theorem 6.1.4. The algebra $\mathbf{U}_{n}^{\mathfrak{c}}$ is a coideal subalgebra of $\mathbf{U}_{n}$, and $\left(\mathbf{U}_{n}, \mathbf{U}_{n}^{\mathfrak{c}}\right)$ forms an affine quantum symmetric pair in the sense of Kolb-Letzter [Ko14]. (see Figure 1 for the relevant involution.)

The following is a variant of [Ko14, Theorem 7.1] in our setting and our notation.

Proposition 6.1.5. For $n=2 r+2$ with $r \geqslant 1$, the $\mathbb{Q}(v)$-algebra $\mathbf{U}_{n}^{\mathfrak{c}}$ has a presentation with generators $\mathbf{e}_{i}, \mathbf{f}_{i}$, and $\mathbf{k}_{i}^{ \pm 1}$ for $i \in[0, r]$ and relations given in Proposition 5.1.2.

Note that the first relation in Proposition 5.1.2 (which is not present in [Ko14]) simply reflects the fact that various quantum affine algebras arising from geometry in this paper are always of level zero.

For $n=2$ (i.e., $r=0$ ), the imbedding $\jmath_{2}: \mathbf{U}_{2}^{\mathfrak{c}} \rightarrow \mathbf{U}_{2}=\mathbf{U}\left(\widehat{\mathfrak{s l}_{2}}\right)$ in $(\underline{6.1 .2})$ is defined by

$$
\mathbf{e}_{0} \mapsto \mathbf{E}_{0}+v^{-1} \mathbf{K}_{0} \mathbf{F}_{1}, \quad \mathbf{f}_{0} \mapsto \mathbf{E}_{1}+v^{-1} \mathbf{K}_{1} \mathbf{F}_{0}, \quad \mathbf{k}_{0} \mapsto \mathbf{K}_{0} \mathbf{K}_{1}^{-1} .
$$

We shall give a presentation for $\mathbf{U}_{2}^{\mathfrak{c}}$, which was excluded from Proposition 6.1.5 above.

Proposition 6.1.6. The $\mathbb{Q}(v)$-algebra $\mathbf{U}_{2}^{\mathfrak{c}}$ has a presentation with generators $\mathbf{e}_{0}, \mathbf{f}_{0}$, and $\mathbf{k}_{0}^{ \pm 1}$ and the following relations.

$$
\begin{gathered}
\mathbf{k}_{0} \mathbf{k}_{0}^{-1}=1, \quad \mathbf{k}_{0} \mathbf{e}_{0}=v^{4} \mathbf{e}_{0} \mathbf{k}_{0}, \quad \mathbf{k}_{0} \mathbf{f}_{0}=v^{-4} \mathbf{f}_{0} \mathbf{k}_{0}, \\
\mathbf{e}_{0}^{3} \mathbf{f}_{0}-\llbracket 3 \rrbracket \mathbf{e}_{0}^{2} \mathbf{f}_{0} \mathbf{e}_{0}+\llbracket 3 \rrbracket \mathbf{e}_{0} \mathbf{f}_{0} \mathbf{e}_{0}^{2}-\mathbf{f}_{0} \mathbf{e}_{0}^{3}=\llbracket 3 \rrbracket !\left(v-v^{-1}\right) \mathbf{e}_{0}\left(\mathbf{k}_{0}-\mathbf{k}_{0}^{-1}\right) \mathbf{e}_{0}, \\
\mathbf{f}_{0}^{3} \mathbf{e}_{0}-\llbracket 3 \rrbracket \mathbf{f}_{0}^{2} \mathbf{e}_{0} \mathbf{f}_{0}+\llbracket 3 \rrbracket \mathbf{f}_{0} \mathbf{e}_{0} \mathbf{f}_{0}^{2}-\mathbf{e}_{0} \mathbf{f}_{0}^{3}=-\llbracket 3 \rrbracket !\left(v-v^{-1}\right) \mathbf{f}_{0}\left(\mathbf{k}_{0}-\mathbf{k}_{0}^{-1}\right) \mathbf{f}_{0} .
\end{gathered}
$$

Here $\llbracket i \rrbracket=\frac{v^{i}-v^{-i}}{v-v^{-1}}$ and $\llbracket a \rrbracket !=\prod_{1 \leqslant i \leqslant a} \llbracket i \rrbracket$.

Proof. Note that $\mathbf{U}_{2}$ is of level zero, so we have $\mathbf{K}_{0} \mathbf{K}_{1}=1$. Thus $\jmath_{n}\left(\mathbf{k}_{0}\right)=\mathbf{K}_{0}^{2}$. From this, we have the identity (6.1.7).

We now prove the identity (6.1.8). Since $\jmath_{n}$ is injective, it suffices to show that (6.1.8) holds after applying $\jmath_{n}$. In other words, it suffices to prove the identity in $\mathbf{U}_{2}$. Let $S\left(\mathbf{e}_{0}, \mathbf{f}_{0}\right)$ denote the left hand side in (6.1.8). We define $S\left(\mathbf{e}_{0}, \mathbf{E}_{1}\right)$ and $S\left(\mathbf{e}_{0}, v^{-1} \mathbf{K}_{1} \mathbf{F}_{0}\right)$ in a similar fashion. By a lengthy calculation involving $4 \times 2^{4}=64$ terms, we have

$$
S\left(\mathbf{e}_{0}, \mathbf{E}_{1}\right)=\llbracket 3 \rrbracket !\left(v-v^{-1}\right) \mathbf{e}_{0} \mathbf{k}_{0} \mathbf{e}_{0} .
$$

Similarly, we have

$$
S\left(\mathbf{e}_{0}, v^{-1} \mathbf{K}_{1} \mathbf{F}_{0}\right)=-\llbracket 3 \rrbracket !\left(v-v^{-1}\right) \mathbf{e}_{0} \mathbf{k}_{0}^{-1} \mathbf{e}_{0} .
$$

So the relation (6.1.8) follows by adding (6.1.10) and (6.1.11). Similarly, one can show Eq. (6.1.9) and we leave the detail to the reader.

Now we invoke [Ko14, Theorem 7.1], which says no additional relations are needed. This finishes the proof.

\subsection{The ALGEBRA $\dot{\mathbf{U}}_{n}^{\mathfrak{c}}$ AND ITS MONOMIAL BASIS}

Let

$$
\mathbb{Z}_{n}^{\mathfrak{c}}=\left\{\lambda=\left(\lambda_{i}\right)_{i \in \mathbb{Z}} \mid \lambda_{i} \in \mathbb{Z}, \lambda_{i}=\lambda_{i+n}, \lambda_{i}=\lambda_{-i}, \forall i, \lambda_{0}, \lambda_{r+1} \text { odd }\right\}
$$


Let $|\lambda|=\lambda_{1}+\ldots+\lambda_{n}$. Define an equivalence relation $\approx$ on $\mathbb{Z}_{n}^{\mathfrak{c}}$ by letting $\lambda \approx \mu$ if and only if $\lambda-\mu=(\ldots, p, p, p, \ldots)$, for some even integer $p$. Let $\mathbb{Z}_{n}^{\mathfrak{c}} / \approx$ be the set of equivalence classes with respect to the equivalence relation $\approx$; and let $\hat{\lambda}$ be the equivalence class of $\lambda$.

Fix $\hat{\lambda} \in \mathbb{Z}_{n}^{\mathfrak{c}} / \approx$, we define the element $1_{\hat{\lambda}} \in \mathbf{U}_{n, \infty}^{\mathfrak{c}}$ as follows. $\left(1_{\hat{\lambda}}\right)_{d}=0$ if $d \not \equiv|\lambda|(\bmod 2 n)$. If $d=|\lambda|+p n$ for some even integer $p$, we have $\left(1_{\hat{\lambda}}\right)_{d}=1_{\lambda+p I}$. Here $\lambda+p I$ is understood as $\lambda+(\ldots, p, p, p, \ldots)$, and $1_{\lambda+p I} \in \mathbf{U}_{n, d}^{\mathfrak{c}}$ is understood to be zero if there is a negative entry in $\lambda+p I$.

Definition 6.2.1. Let $\dot{\mathbf{U}}_{n}^{\mathfrak{c}}$ be the $\mathbf{U}_{n}^{\mathfrak{c}}$-bimodule in $\mathbf{U}_{n, \infty}^{\mathfrak{c}}$ generated by $1_{\hat{\lambda}}$ for all $\hat{\lambda} \in \mathbb{Z}_{n}^{\mathfrak{c}} / \approx$.

It is clear that $\dot{\mathbf{U}}_{n}^{\mathfrak{c}}$ is a subalgebra of $\mathbf{U}_{n, \infty}^{\mathfrak{c}}$ generated by $1_{\hat{\lambda}}, \mathbf{e}_{i} 1_{\hat{\lambda}}$ and $\mathbf{f}_{i} 1_{\hat{\lambda}}$ for all $i \in[0, r]$ and $\hat{\lambda} \in \mathbb{Z}_{n}^{\mathfrak{c}} / \approx$. Similarly, we define the $\mathcal{A}$-subalgebra ${ }_{\mathcal{A}} \dot{\mathbf{U}}_{n}^{\mathfrak{c}}$ of $\mathbf{U}_{n, \infty}^{\mathfrak{c}}$ generated by $\mathbf{e}_{i}^{(a)} 1_{\hat{\lambda}}$ and $\mathbf{f}_{i}^{(a)} 1_{\hat{\lambda}}$, for all $i \in[0, r]$ and $a \in \mathbb{N}$. So we have $\mathbb{Q}(v) \otimes_{\mathcal{A}}{ }_{\mathcal{A}} \dot{\mathbf{U}}_{n}^{\mathfrak{c}}=\dot{\mathbf{U}}_{n}^{\mathfrak{c}}$. The bar involution on $\mathbf{U}_{n, \infty}^{\mathfrak{c}}$ induces a bar involution on $\dot{\mathbf{U}}_{n}^{\mathfrak{c}}$, which we denote by ${ }^{-}: \dot{\mathbf{U}}_{n}^{\mathfrak{c}} \longrightarrow \dot{\mathbf{U}}_{n}^{\mathfrak{c}}$. Note that it leaves the elements $\mathbf{e}_{i}^{(a)} 1_{\hat{\lambda}}$ and $\mathbf{f}_{i}^{(a)} 1_{\hat{\lambda}}$ fixed, and hence we have ${ }^{-}:{ }_{\mathcal{A}} \dot{\mathbf{U}}_{n}^{\mathfrak{c}} \longrightarrow{ }_{\mathcal{A}} \dot{\mathbf{U}}_{n}^{\mathfrak{c}}$.

We denote

$$
\begin{aligned}
& \widetilde{\Xi}_{n}=\left\{A=\left(a_{i j}\right) \in \operatorname{Mat}_{\mathbb{Z} \times \mathbb{Z}}(\mathbb{Z}) \mid a_{0,0}, a_{r+1, r+1} \in 2 \mathbb{Z}+1,\right. \\
& \left.\qquad a_{i j} \geqslant 0(i \neq j), a_{i j}=a_{-i,-j}=a_{i+n, j+n}(\forall i, j)\right\}, \\
& \widetilde{\Xi}_{n}^{a p}=\left\{A \in \widetilde{\Xi}_{n} \mid A \text { is aperiodic }\right\} .
\end{aligned}
$$

For $A \in \widetilde{\Xi}_{n}$, we shall denote by

$$
|A|=d
$$

if $\sum_{i=i_{0}+1}^{i_{0}+n} \sum_{j \in \mathbb{Z}} a_{i j}=2 d+2$ for some (or each) $i_{0} \in \mathbb{Z}$. We set, for $d \in \mathbb{Z}$,

$$
\widetilde{\Xi}_{n, d}=\left\{A \in \widetilde{\Xi}_{n}|| A \mid=d\right\}, \quad \widetilde{\Xi}_{n}=\sqcup_{d} \widetilde{\Xi}_{n, d} .
$$

Also clearly we have $\Xi_{n, d} \subset \widetilde{\Xi}_{n, d}$.

We define an equivalence relation $\approx$ on $\widetilde{\Xi}_{n}^{a p}$ by

$$
A \approx B \text { iff } A-B=p I_{n} \text {, for some even integer } p,
$$

where $I_{n}=\sum_{1 \leqslant i \leqslant n} E^{i i}$, and let $\widehat{A}$ be the equivalence class of $A$. Whenever there causes no ambiguity, we write $I$ for $I_{n}$. We define $\operatorname{ro}(\widehat{A})=\widehat{\operatorname{ro}(A)}$ and $\operatorname{co}(\widehat{A})=\widehat{\operatorname{co}(A)}$, and they are elements in $\mathbb{Z}_{n}^{\mathfrak{c}} / \approx$. We can then define the element $\zeta_{\hat{A}}$ in $\dot{\mathbf{U}}_{n}^{\mathfrak{c}}$ by $\left(\zeta_{\hat{A}}\right)_{d}=0$ unless $d=|A|$ $\bmod 2 n$, and if $|A|=d+p / 2 n$ for some even integer $p,\left(\zeta_{\widehat{A}}\right)_{d}=\zeta_{A+p I}$, where $\zeta_{A+p I}$ is the monomial basis attached to $A+p I$ in Theorem 5.4.3. Since $\phi_{d, d-n}^{\mathfrak{c}}\left(\zeta_{A+p I}\right)=\zeta_{A+(p-2) I}$, we see that $\zeta_{\hat{A}} \in \dot{\mathbf{U}}_{n}^{\mathfrak{c}}$.

The following linear independence is reduced to the counterpart at the Schur algebra level, by an argument similar to [LW15, Theorem 5.5].

Proposition 6.2.2. The set $\left\{\zeta_{\widehat{A}} \mid \widehat{A} \in \widetilde{\Xi}_{n}^{a p} / \approx\right\}$ is linearly independent.

To show that $\zeta_{\hat{A}}$ is indeed a basis for $\dot{\mathbf{U}}_{n}^{\mathfrak{c}}$, let us take a closer look at the behavior of the monomials at the Schur algebra level. For simplicity, we write $\mathbf{f}_{n-(i+1)}$ for $\mathbf{e}_{i}$ for all $i \in[0, r]$. 
For $\lambda \in \Lambda_{n, d}^{\mathfrak{c}}$ and a pair $(\mathbf{i}, \mathbf{a})$ where $\mathbf{i}=\left(i_{1}, \ldots, i_{s}\right)$ and $\mathbf{a}=\left(a_{1}, \ldots, a_{s}\right)$ with $0 \leqslant i_{j} \leqslant n$ and $a_{j} \in \mathbb{N}$ for all $j$, we set

$$
{ }_{d} \mathrm{M}_{\mathbf{i}, \mathbf{a}, \lambda}=\mathbf{f}_{i_{1}}^{\left(a_{1}\right)} \mathbf{f}_{i_{2}}^{\left(a_{2}\right)} \cdots \mathbf{f}_{i_{s}}^{\left(a_{s}\right)} 1_{\lambda} \in \mathbf{U}_{n, d}^{\mathfrak{c}},
$$

where $1_{\lambda}=\left[D_{\lambda}\right]_{d}$ is the standard basis element of the diagonal matrix whose diagonal is $\lambda$. Then ${ }_{d} \mathbf{M}_{\mathbf{i}, \mathbf{a}, \lambda}$ exhaust all possible monomials in $\mathbf{U}_{n, d}^{\mathfrak{c}}$. The following proposition is crucial in showing that the various $\xi_{\hat{A}}$ forms a basis for $\dot{\mathbf{U}}_{n}^{\mathfrak{c}}$. Recall that $I=\sum_{1 \leqslant i \leqslant n} E_{\theta}^{i i}$.

Proposition 6.2.3. Fix a triple $(\mathbf{i}, \mathbf{a}, \lambda)$ with $|\lambda|=d$. There is a finite subset $\mathcal{I}_{\mathbf{i}, \mathbf{a}, \lambda}$ of $\left\{A \in \widetilde{\Xi}_{n}^{a p}|| A \mid=d\right\}$ such that

$$
d+p n M_{\mathbf{i}, \mathbf{a}, \lambda+2 p I}=\sum_{A \in \mathcal{I}_{\mathbf{i}, \mathbf{a}, \lambda}} c_{A} \zeta_{2 p} A, \forall p, \text { where } c_{A} \in \mathcal{A} \text { is independent of } p .
$$

Proof. By the multiplication formula for simple generators, we see that the standard basis element $[A]$, possibly periodic, appearing in ${ }_{d+p n} \mathbf{M}_{\mathbf{i}, \mathbf{a}, \lambda+2 p I}$ is stabilized for $p \gg 0$. In other words, there is a finite set $\mathcal{J}_{\mathbf{i}, \mathbf{a}, \lambda}$ in $\widetilde{\Xi}_{n}$ consisting of certain $A$ subject to $|A|=d$ and

$$
d+p n \mathbf{M}_{\mathbf{i}, \mathbf{a}, \lambda+2 p I}=\sum_{A \in \mathcal{J}_{\mathbf{i}, \mathbf{a}, \lambda}} g_{A, p}[A+2 p I], \quad \forall p
$$

where $g_{A, p} \in \mathcal{A}$ depends on $p$ in general.

Note that $\mathcal{J}_{\mathbf{i}, \mathbf{a}, \lambda}$ can be constructed in the following way. Fix a $p$ large enough, so that when we multiply out the monomial ${ }_{d+p n} \mathrm{M}_{\mathbf{i}, \mathbf{a}, \lambda+2 p I}$ in terms of standard basis, we do not miss a term because that term has a negative entry in its diagonal. Collect all the matrices, say $A$, parametrizing the standard basis element appearing in ${ }_{d+p n} \mathrm{M}_{\mathbf{i}, \mathbf{a}, \lambda+2 p I}$, and further throwing into this set all matrices $B$ such that $B<_{\text {alg }} A$. This resulting set is again finite. $\mathcal{J}_{\mathbf{i}, \mathbf{a}, \lambda}$ is then defined to be the set of matrices obtained by subtracting the matrices in the previous set by $2 p I$.

Let $\mathcal{I}_{\mathbf{i}, \mathbf{a}, \lambda}$ be the subset of $\mathcal{J}_{\mathbf{i}, \mathbf{a}, \lambda}$ consisting of aperiodic elements. It follows by Theorem 5.4 .3 that

$$
{ }_{d+p n} \mathrm{M}_{\mathbf{i}, \mathbf{a}, \lambda+2 p I}=\sum_{A \in \mathcal{I}_{\mathbf{i}, \mathbf{a}, \lambda}} c_{A, p} \zeta_{2 p} A, \quad \forall p,
$$

where $c_{A, p} \in \mathcal{A}$ depends on $p$ in general.

By definition, we have

$$
\begin{aligned}
\phi_{d+p n, d+p n-n}^{\mathfrak{c}}\left({ }_{d+p n} \mathrm{M}_{\mathbf{i}, \mathbf{a}, \lambda+2 p I}\right) & ={ }_{d+p n-n} \mathrm{M}_{\mathbf{i}, \mathbf{a}, \lambda+2 p I-2 I}, \\
\phi_{d+p n, d+p n-n}^{\mathfrak{c}}\left(\zeta_{2 p} A\right) & =\zeta_{p-2} A, \quad \forall p .
\end{aligned}
$$

This implies that

$$
c_{A, p}=c_{A, p-1}, \quad \text { if } \zeta_{2(p-1)} A \neq 0 \in \mathbf{U}_{n, d+(p-1) n}^{\mathfrak{c}} .
$$

For large enough $p, \zeta_{2(p-1)} A$ is obviously nonzero, and so $c_{A, p}=c_{A}$ is independent of $p \gg 0$. Recall that the set $\mathcal{I}_{\mathbf{i}, \mathbf{a}, \lambda}$ is finite. So we can find a $p_{0}$ such that $c_{A, p}=c_{A}$ for all $p \geqslant p_{0}$ and for all $A \in \mathcal{I}_{\mathbf{i}, \mathbf{a}, \lambda}$. The proposition is thus proved.

Now we return from Lusztig algebras to the algebra $\dot{\mathbf{U}}_{n}^{\mathfrak{c}}$.

Proposition 6.2.4. The set $\left\{\zeta_{\hat{A}} \mid \widehat{A} \in \widetilde{\Xi}_{n}^{a p} / \approx\right\}$ forms a basis for $\dot{\mathbf{U}}_{n}^{\mathfrak{c}}$ and an $\mathcal{A}$-basis for ${ }_{\mathcal{A}} \dot{\mathbf{U}}_{n}^{\mathfrak{c}}$. 
Proof. Similar to the element ${ }_{d} \mathbf{M}_{\mathbf{i}, \mathbf{a}, \lambda}$, we can define its limit version $\mathbf{M}_{\mathbf{i}, \mathbf{a}, \hat{\lambda}}$ in $\dot{\mathbf{U}}_{n}^{\mathfrak{c}}$. Moreover, these monomials exhaust all the possible monomials in $\dot{\mathbf{U}}_{n}^{\mathfrak{c}}$. The proposition now follows from Proposition 6.2.3.

\subsection{BILINEAR FORM ON $\dot{\mathbf{U}}_{n}^{\mathfrak{c}}$}

Recall that for $i \in[0, r], \mathbf{e}_{i}=\sum[A]$ where $A-E_{\theta}^{i+1, i}$ is diagonal, $\mathbf{f}_{i}=\sum[A]$ where $A-E_{\theta}^{i, i+1}$ is diagonal, and $\mathbf{k}_{i}=\sum_{\lambda \in \Lambda_{n, d}^{\mathrm{c}}} v^{\lambda_{i+1}-\lambda_{i}} 1_{\lambda}$.

Imitating McGerty [Mc12] in affine type $A$, we define a bilinear form $\langle\cdot, \cdot\rangle_{d}$ on $\mathbf{S}_{n, d}^{\mathfrak{c}}$ as follows:

$$
\left\langle[A],\left[A^{\prime}\right]\right\rangle_{d}=\delta_{A, A^{\prime}} v^{-2 d_{A^{t}}} \# X_{A^{t}}^{L^{\prime}},
$$

where $L^{\prime} \in \mathcal{X}_{n, d}^{\mathfrak{c}}\left(\operatorname{ro}\left(A^{t}\right)\right)$. With the help of the identity (4.2.10), the same argument as in [Mc12, Proposition 3.2] gives us the following.

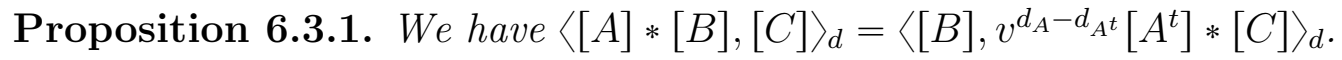

Corollary 6.3.2. For all $i \in[0, r]$, we have the following:

(1) $\left\langle\mathbf{e}_{i}\left[A_{1}\right],\left[A_{2}\right]\right\rangle_{d}=\left\langle\left[A_{1}\right], v \mathbf{k}_{i} \mathbf{f}_{i}\left[A_{2}\right]\right\rangle_{d}$.

(2) $\left\langle\mathbf{f}_{i}\left[A_{1}\right],\left[A_{2}\right]\right\rangle_{d}=\left\langle\left[A_{1}\right], v^{-1} \mathbf{e}_{i} \mathbf{k}_{i}^{-1}\left[A_{2}\right]\right\rangle_{d}$.

(3) $\left\langle\mathbf{k}_{i}\left[A_{1}\right],\left[A_{2}\right]\right\rangle_{d}=\left\langle\left[A_{1}\right], \mathbf{k}_{i}\left[A_{2}\right]\right\rangle_{d}$.

Proof. We prove (1). If $A-E_{\theta}^{i+1, i}$ is diagonal for some $i \in[0, r]$, then

$$
d_{A}=\operatorname{co}(A)_{i+1} \quad \text { and } \quad d_{A^{t}}=\operatorname{ro}(A)_{i}=\operatorname{co}(A)_{i}-1 .
$$

Hence $d_{A}-d_{A^{t}}=\operatorname{co}(A)_{i+1}-\operatorname{co}(A)_{i}+1$. Thus, we have

$$
v \mathbf{k}_{i}\left(L, L^{\prime}\right)=\delta_{L, L^{\prime}} v^{1+\operatorname{co}(A)_{i+1}-\operatorname{co}(A)_{i}}=\delta_{L, L^{\prime}} v^{d_{A}-d_{A^{t}}}, \forall L, L^{\prime} \in \mathcal{X}_{n, d}^{\mathfrak{c}}(\operatorname{co}(A)),
$$

which implies (1).

We now prove (2). If $A-E_{\theta}^{i, i+1}$ is diagonal for some $i \in[0, r]$, then

$$
d_{A}=\operatorname{co}(A)_{i} \quad \text { and } \quad d_{A^{t}}=\operatorname{ro}(A)_{i+1}=\operatorname{co}(A)_{i+1}-1-\delta_{i, 0}-\delta_{i, n} .
$$

So $d_{A}-d_{A^{t}}=\operatorname{co}(A)_{i}-\operatorname{co}(A)_{i+1}+1+\delta_{i, 0}+\delta_{i, n}$. Hence, if $\left(L, L^{\prime}\right)$ subject to $L \in \mathcal{X}_{n, d}^{\mathfrak{c}}(\operatorname{co}(A))$, $L_{i} \subseteq L_{i}^{\prime}, L_{j}=L_{j}^{\prime}$ for all $j \in[0, r] \backslash\{i\}$, then

$$
\begin{aligned}
v^{d_{A}-d_{A^{t}}} \mathbf{e}_{i}\left(L, L^{\prime}\right) & =v^{1+\delta_{i, 0}+\delta_{i, n}} \mathbf{k}_{i}^{-1} \mathbf{e}_{i}\left(L, L^{\prime}\right) \\
& =v^{1+\delta_{i, 0}+\delta_{i, n}} v^{-2-\delta_{i, 0}-\delta_{i, n}} \mathbf{e}_{i} \mathbf{k}_{i}^{-1}\left(L, L^{\prime}\right)=v^{-1} \mathbf{e}_{i} \mathbf{k}_{i}^{-1}\left(L, L^{\prime}\right) .
\end{aligned}
$$

Part (2) follows.

Part (3) follows from the fact that $d_{A}=d_{A^{t}}=0$ if $A$ is diagonal.

The same argument as in [Mc12] shows that there is a well-defined bilinear form $\langle\cdot, \cdot\rangle$ on $\dot{\mathbf{U}}_{n}^{\mathfrak{c}}$ given by

$$
\langle x, y\rangle=\sum_{d=1}^{n} \lim _{p \rightarrow \infty}\left\langle x_{d+p n}, y_{d+p n}\right\rangle_{d+p n}, \quad \forall x=\left(x_{d}\right), y=\left(y_{d}\right) \in \dot{\mathbf{U}}_{n}^{\mathfrak{c}} .
$$

Remark 6.3.3. The same adjointness property as in Proposition 6.3.1 holds for the bilinear form $\langle\cdot, \cdot\rangle$ on $\dot{\mathbf{U}}_{n}^{\mathfrak{c}}$. 


\subsection{The CANONICAL BASIS OF $\dot{\mathbf{U}}_{n}^{\mathfrak{c}}$ AND POSITIVITY}

As we have set up all the preliminary preparation, the constructions and properties of the canonical basis for $\dot{\mathbf{U}}_{n}^{\mathfrak{c}}$ can be established without further difficulty. Actually as the technical proofs for the intermediate steps are literally the same as in the affine type $A$ setting [Mc12] and/or in the finite type $B / C$ setting [LW15, FL15], we will formulate the statements while referring to those papers for detailed proofs.

With the help of the bilinear form $\langle\cdot, \cdot\rangle$ and Theorem 5.4.3, the same arguments as in [Mc12], or [LW15] prove the following.

Proposition 6.4.1. For any $A \in \Xi_{n, d}^{a p}$, we have

$$
\phi_{d+p n, d+(p-1) n}^{\mathfrak{c}}\left(\left\{{ }_{2 p} A\right\}_{d+p n}\right)=\left\{{ }_{2 p-2} A\right\}_{d+(p-1) n}, \quad \forall p \gg 0 .
$$

Moreover, we have

$$
\left\{{ }_{2 p} A\right\}_{d+p n}=\zeta_{2 p} A+\sum_{B \in \widetilde{\Xi}_{n}^{a p}: B<A} c_{A, B, p} \zeta_{2 p} B
$$

with $c_{A, B, p} \in \mathcal{A}$ independent of $p$ for $p \gg 0$.

Recall $\widetilde{\Xi}_{n}$ and $\widetilde{\Xi}_{n}^{a p}$ from $(\underline{6.2 .2})$.

Definition 6.4.2. For any $\widehat{A} \in \widetilde{\Xi}_{n}^{a p} / \approx$, an element $b_{\widehat{A}} \in \dot{\mathbf{U}}_{n}^{\mathfrak{c}}$ is defined as follows: $\left(b_{\widehat{A}}\right)_{d}=0$ if $d \neq|A| \bmod 2 n$; If $|A|=d+$ sn for some integer $s$, we set

$$
\left(b_{\widehat{A}}\right)_{d+s n+p n}=\left\{{ }_{2 p} A\right\}_{d+s n+p n}, \quad \forall p \geqslant p_{0}, \text { for some fixed } p_{0},
$$

and for general $q<p_{0}$, we set $\left(b_{\widehat{A}}\right)_{d+s n+q n}=\phi_{d+s n+p_{0} n, d+r n+q n}^{\mathfrak{c}}\left(\left\{2 p_{0} A\right\}_{d+s n+p_{0} n}\right)$.

The fact that $b_{\hat{A}}$ as defined above lies in $\dot{\mathbf{U}}_{n}^{\mathfrak{c}}$ follows from Proposition 6.4.1. Moreover, $\zeta_{\hat{A}}=b_{\widehat{A}}+$ lower terms. The next theorem now follows from the existence of the monomial basis $\left\{\zeta_{\widehat{A}}\right\}$ for $\dot{\mathbf{U}}_{n}^{\mathfrak{c}}$; cf. Proposition 6.2.4,

Theorem 6.4.3. The set $\dot{\mathbf{B}}_{n}^{\mathfrak{c}}:=\left\{b_{\widehat{A}} \mid \widehat{A} \in \widetilde{\Xi}_{n}^{a p} / \approx\right\}$ forms a basis for $\dot{\mathbf{U}}_{n}^{\mathfrak{c}}$.

The basis $\dot{\mathbf{B}}_{n}^{\mathfrak{c}}$ is called the canonical basis of $\dot{\mathbf{U}}_{n}^{\mathfrak{c}}$.

As a consequence, we deduce formally the following results by the same arguments in [LW15] and [FL15].

Proposition 6.4.4. The signed canonical basis $\left\{ \pm b_{\widehat{A}} \mid \widehat{A} \in \widetilde{\Xi}_{n}^{a p} / \approx\right\}$ is characterized by the bar-invariance, integrality (i.e. $b_{\widehat{A}} \in{ }_{\mathcal{A}} \dot{\mathbf{U}}_{n}^{\mathfrak{c}}$ ), and almost orthonormality (i.e., $\left\langle b_{\widehat{A}}, b_{\hat{A}^{\prime}}\right\rangle=\delta_{\hat{A}, \hat{A}^{\prime}}$ $\left.\bmod v^{-1} \mathbb{Z}\left[\left[v^{-1}\right]\right]\right)$.

The canonical basis of $\dot{\mathbf{U}}_{n}^{\mathfrak{c}}$ enjoys several remarkable positivity properties as follows. The proofs use the same arguments as in [LW15] and [FL15]. In particular, for the positivity with respect to comultiplication, the positivity of the canonical basis in the Lusztig algebra $\mathbf{U}_{n, d}^{\mathfrak{c}}$ as in Proposition 5.4.4 is used.

Theorem 6.4.5. The structure constants of the canonical basis $\dot{\mathbf{B}}_{n}^{\mathbf{c}}$ lie in $\mathbb{N}\left[v, v^{-1}\right]$ with respect to the multiplication and comultiplication, and in $v^{-1} \mathbb{N}\left[\left[v^{-1}\right]\right]$ with respect to the bilinear pairing. 


\subsection{Another presentation of the Algebra $\dot{\mathbf{U}}_{n}^{\mathfrak{c}}$}

We shall give a more familiar description of the algebra $\dot{\mathbf{U}}_{n}^{\mathfrak{c}}$. We start with introducing the limit version of the imbeddings $\jmath_{n, d}$.

Recall $\mathbb{Z}_{n}$ from (2.3.1) and $\mathbb{Z}_{n}^{\mathfrak{c}}$ from (6.2.1), and there is an inclusion $\mathbb{Z}_{n}^{\mathfrak{c}} \subset \mathbb{Z}_{n}$. Recall the notation $\models$ from (5.4.4), and we extend it further to $\mathbb{Z}_{n}^{\mathfrak{c}} \times \mathbb{Z}_{n}$ as follows. Given a pair $\left(\lambda, \lambda^{\prime}\right) \in \mathbb{Z}_{n}^{\mathfrak{c}} \times \mathbb{Z}_{n}$, we write $\lambda^{\prime} \models \lambda$ if

$$
\lambda_{i}=\lambda_{i}^{\prime}+\lambda_{n-i}^{\prime}+\delta_{i, n}+\delta_{i, r+1}, \quad \forall 1 \leqslant i \leqslant n .
$$

We write $\bar{\lambda}^{\prime} \models \hat{\lambda}$ if $\lambda^{\prime} \models \tilde{\lambda}$ for some $\tilde{\lambda}$ in the equivalence class $\hat{\lambda}$ and the notation $\bar{\lambda}$ is defined in Section 2.3. (In this case, we shall assume that $\lambda^{\prime} \models \lambda$.)

$\operatorname{Recall}_{\bar{\mu}}\left(\dot{\mathbf{U}}_{n}\right)_{\bar{\lambda}}$ from Section 2.3, We set $\hat{\mu}\left(\dot{\mathbf{U}}_{n}^{\mathfrak{c}}\right)_{\hat{\lambda}}=1_{\hat{\mu}} \dot{\mathbf{U}}_{n}^{\mathfrak{c}} 1_{\hat{\lambda}}$. For a quadruple $\left(\hat{\lambda}, \widehat{\mu}, \bar{\lambda}^{\prime}, \bar{\mu}^{\prime}\right)$ such that $\bar{\lambda}^{\prime} \models \widehat{\lambda}$ and $\bar{\mu}^{\prime} \models \widehat{\mu}$, we define a linear map

$$
J_{\bar{\lambda}^{\prime}, \bar{\mu}^{\prime}, \hat{\lambda}, \hat{\mu}}: \hat{\mu}\left(\dot{\mathbf{U}}_{n}^{\mathfrak{c}}\right)_{\hat{\lambda}} \longrightarrow \bar{\mu}^{\prime}\left(\dot{\mathbf{U}}_{n}\right)_{\bar{\lambda}^{\prime}}
$$

to be the composition

$$
\widehat{\mu}\left(\dot{\mathbf{U}}_{n}^{\mathfrak{c}}\right)_{\hat{\lambda}} \hookrightarrow \dot{\mathbf{U}}_{n}^{\mathfrak{c}} \stackrel{\jmath_{n}}{\longrightarrow} \mathbf{U}_{n, \infty} \rightarrow \bar{\mu}^{\prime}\left(\dot{\mathbf{U}}_{n}\right)_{\bar{\lambda}^{\prime}}
$$

where the first map is a natural inclusion and the third one is the projection. Set

$$
J_{\hat{\lambda}, \hat{\mu}}=\prod_{\bar{\lambda}^{\prime} \models \hat{\lambda}, \bar{\mu}^{\prime} \models \widehat{\mu}} J_{\bar{\lambda}^{\prime}, \bar{\mu}^{\prime}, \hat{\lambda}, \hat{\mu}}: \hat{\mu}\left(\dot{\mathbf{U}}_{n}^{\mathfrak{c}}\right)_{\hat{\lambda}} \longrightarrow \prod_{\bar{\lambda}^{\prime} \models \hat{\lambda}, \bar{\mu}^{\prime} \models \widehat{\mu}} \bar{\mu}^{\prime}\left(\dot{\mathbf{U}}_{n}\right)_{\bar{\lambda}^{\prime}} .
$$

Recall the imbedding $\jmath_{n}: \mathbf{U}_{n, \infty}^{\mathfrak{c}} \rightarrow \mathbf{U}_{n, \infty}$ from Section 6.1. We have

$$
J_{n}\left(\hat{\mu}\left(\dot{\mathbf{U}}_{n}^{\mathfrak{c}}\right)_{\hat{\lambda}}\right) \subseteq \prod_{\bar{\lambda}^{\prime} \models \widehat{\lambda}, \bar{\mu}^{\prime} \models \widehat{\mu}} \bar{\mu}^{\prime}\left(\dot{\mathbf{U}}_{n}\right)_{\bar{\lambda}^{\prime}}
$$

The injectivity of $\jmath_{n}$ implies that the homomorphism $\jmath_{\hat{\lambda}, \hat{\mu}}$ is injective.

Now a modified form of $\mathbf{U}_{n}^{\mathfrak{c}}$, denoted by $\dot{\mathbf{U}}_{n \text {,alg }}^{\mathfrak{c}}$, can be defined algebraically in a standard way as

$$
\dot{\mathbf{U}}_{n, \mathrm{alg}}^{\mathfrak{c}}=\oplus_{\widehat{\mu}, \hat{\lambda} \in \mathbb{Z}_{n}^{\mathfrak{c}} / \approx \hat{\mu}}\left(\mathbf{U}_{n, \text { alg }}^{\mathfrak{c}}\right)_{\hat{\lambda}}
$$

where

$$
\widehat{\mu}\left(\dot{\mathbf{U}}_{n, \text { alg }}^{\mathfrak{c}}\right)_{\hat{\lambda}}=\mathbf{U}_{n}^{\mathfrak{c}} /\left(\sum_{0 \leqslant i \leqslant r}\left(\mathbf{k}_{i}-v^{-\mu_{i}+\mu_{i+1}}\right) \mathbf{U}_{n}^{\mathfrak{c}}+\sum_{0 \leqslant i \leqslant r} \mathbf{U}_{n}^{\mathfrak{c}}\left(\mathbf{k}_{i}-v^{-\lambda_{i}+\lambda_{i+1}}\right)\right) .
$$

The algebra homomorphism $\jmath_{n}: \mathbf{U}_{n}^{\mathfrak{c}} \rightarrow \mathbf{U}_{n}$ then induces a linear map

$$
\tilde{J}_{\bar{\lambda}^{\prime}, \bar{\mu}^{\prime}, \hat{\lambda}, \hat{\mu}}: \hat{\mu}\left(\dot{\mathbf{U}}_{n, \operatorname{alg}}^{\mathfrak{c}}\right)_{\hat{\lambda}} \longrightarrow \bar{\mu}^{\prime}\left(\dot{\mathbf{U}}_{n}\right)_{\bar{\lambda}^{\prime}}
$$

such that the following diagram commutes:

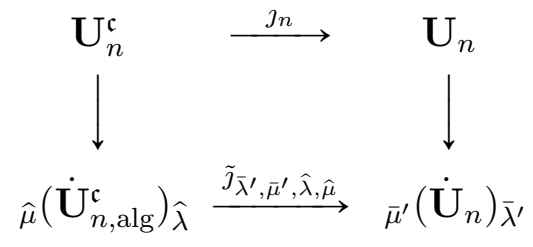


Set

$$
\tilde{j}_{\hat{\lambda}, \hat{\mu}}=\prod_{\substack{\bar{\lambda}^{\prime} \in \hat{\lambda} \\ \bar{\mu}^{\prime} \models \hat{\mu}}} \tilde{j}_{\bar{\lambda}^{\prime}, \bar{\mu}^{\prime}, \hat{\lambda}, \hat{\mu}}: \hat{\mu}\left(\dot{\mathbf{U}}_{n, \operatorname{alg}}^{\mathrm{c}}\right)_{\hat{\lambda}} \longrightarrow \prod_{\substack{\bar{\lambda}^{\prime} \in \hat{\lambda} \\ \bar{\mu}^{\prime} \models \hat{\mu}}} \bar{\mu}^{\prime}\left(\dot{\mathbf{U}}_{n}\right)_{\bar{\lambda}^{\prime}} \cdot
$$

Since $\jmath_{n}$ is injective, $\tilde{\jmath}_{\hat{\lambda}, \widehat{\mu}}$ is injective.

By definition, there exists a unique linear map

$$
\phi_{d, \mathrm{alg}, \hat{\mu}, \hat{\lambda}}: \hat{\mu}\left(\dot{\mathbf{U}}_{n, \mathrm{alg}}^{\mathfrak{c}}\right)_{\hat{\lambda}} \longrightarrow \oplus_{\mathbf{b} \in \hat{\mu}, \mathbf{a} \in \hat{\lambda}} \mathbf{U}_{n, d}^{\mathfrak{c}}(\mathbf{b}, \mathbf{a}),
$$

where $\mathbf{U}_{n, d}^{\mathfrak{c}}(\mathbf{b}, \mathbf{a})=1_{\mathbf{b}}\left(\mathbf{U}_{n, d}^{\mathfrak{c}}\right) 1_{\mathbf{a}}$, such that the following diagram commutes:

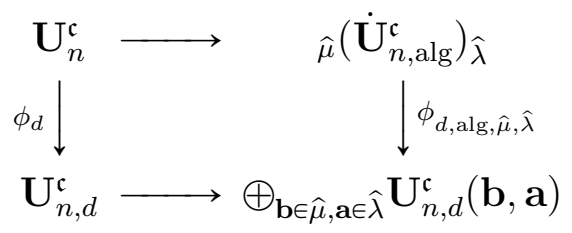

From this we have constructed an algebra homomorphism

$$
\phi_{d, \text { alg }}:=\oplus_{\widehat{\mu}, \hat{\lambda} \in \mathbb{Z}_{n}^{\mathfrak{c}} / \approx} \phi_{d, \text { alg, }, \hat{\mu}, \hat{\lambda}}: \dot{\mathbf{U}}_{n, \text { alg }}^{\mathfrak{c}} \longrightarrow \mathbf{U}_{n, d}^{\mathfrak{c}} \text {. }
$$

Since $\phi_{d, \text { alg }}$ commutes with the transfer maps, i.e., $\phi_{d-n, \text { alg }}=\phi_{d, d-n} \phi_{d, \text { alg }}$. we obtain an algebra homomorphism $\psi: \dot{\mathbf{U}}_{n \text {,alg }}^{\mathfrak{c}} \rightarrow \mathbf{U}_{n, \infty}^{\mathfrak{c}}$. Observe that the image of this homomorphism is exactly $\dot{\mathbf{U}}_{n}^{\mathfrak{c}}$ by considering the image of the idempotents $1_{\hat{\lambda}}$. Therefore, we have a surjective algebra homomorphism: $\psi: \dot{\mathbf{U}}_{n, \text { alg }}^{\mathfrak{c}} \longrightarrow \dot{\mathbf{U}}_{n}^{\mathfrak{c}}$. By restriction, we have $\psi_{\widehat{\mu}, \hat{\lambda}}: \hat{\mu}\left(\dot{\mathbf{U}}_{n, \text { alg }}^{\mathfrak{c}}\right)_{\hat{\lambda}} \rightarrow \hat{\mu}\left(\dot{\mathbf{U}}_{n}^{\mathfrak{c}}\right)_{\hat{\lambda}}$, for various $\widehat{\mu}, \hat{\lambda}$. Since $J_{\hat{\lambda}, \widehat{\mu}}$ and $\tilde{\jmath}_{\hat{\lambda}, \hat{\mu}}$ are injective, and $\tilde{\jmath}_{\hat{\lambda}, \hat{\mu}}=J_{\hat{\lambda}, \hat{\mu}} \circ \psi_{\widehat{\mu}, \hat{\lambda}}$, we conclude that $\psi_{\widehat{\mu}, \hat{\lambda}}$ and hence $\psi$ is injective. Summarizing, we have established the following.

Proposition 6.5.1. The map $\psi: \dot{\mathbf{U}}_{n, a l g}^{\mathfrak{c}} \rightarrow \dot{\mathbf{U}}_{n}^{\mathfrak{c}}$ is an algebra isomorphism.

Therefore, a presentation of $\dot{\mathbf{U}}_{n}^{\mathfrak{c}}$ is reduced to finding a presentation of $\dot{\mathbf{U}}_{n, \text { alg }}^{\mathfrak{c}}$, and the latter can be obtained by modifying the definition/presentation of $\mathbf{U}_{n}^{\mathfrak{c}}$ as given in Propositions 6.1.5 and 6.1.6. in a way similar to Lusztig's presentation for modified quantum groups [Lu93]. The finite type counterpart of a presentation of $\dot{\mathbf{U}}_{n, \text { alg }}^{\mathfrak{c}}$ can be found in [BKLW14]. 


\section{CHAPTER 7}

\section{A second coideal subalgebra of quantum affine $\mathfrak{s l}_{\mathfrak{n}}$}

In this chapter, setting $\mathfrak{n}=n-1$ we consider a subvariety of $\mathcal{X}_{n, d}^{\mathfrak{c}}$, and study its corresponding convolution algebra $\mathbf{S}_{\mathfrak{n}, d}^{\jmath v}$ which is a subalgebra of $\mathbf{S}_{n, d}^{\mathfrak{c}}$. We introduce Lusztig

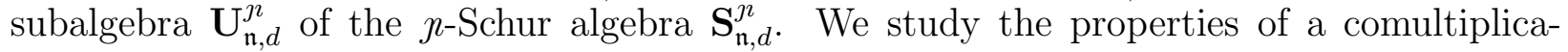
tion on $\mathbf{U}_{\mathfrak{n}, d}^{\jmath \imath}$, which allow us to form a projective system $\left\{\left(\mathbf{U}_{\mathfrak{n}, d}^{\jmath n}, \phi_{d, d-\mathfrak{n}}^{\jmath n}\right)\right\}_{d \geqslant 0}$ and then two distinguished algebras $\mathbf{U}_{\mathfrak{n}}^{\jmath n}$ and $\dot{\mathbf{U}}_{\mathfrak{n}}^{\jmath n}$. We show that $\left(\mathbf{U}\left(\widehat{\mathfrak{s l}}_{\mathfrak{n}}\right), \mathbf{U}_{\mathfrak{n}}^{\jmath n}\right)$ forms an affine quantum symmetric pair. The canonical basis of $\dot{\mathbf{U}}_{\mathfrak{n}}^{\jmath n}$ is established and shown to admit positivity with respect to multiplication, comultiplication, and a bilinear pairing.

Recall $n=2 r+2$, and we now set

$$
\mathfrak{n}=n-1=2 r+1 \quad(r \geqslant 1) .
$$

\subsection{The Schur algebras of TyPe $\imath$}

We shall construct Schur algebras $\mathbf{S}_{\mathfrak{n}, d}^{j n}$ and Lusztig algebras $\mathbf{U}_{\mathfrak{n}, d}^{n n}$. These algebras are defined as the affine counterpart of [BKLW14], and many basic properties of these algebras are established following [FL15, Section 5].

Recall the set $\Xi_{n, d}$ from (4.2.1). We introduce a subset $\Xi_{\mathfrak{n}, d}^{\mathfrak{j}}$ which consists of matrices $A \in \Xi_{n, d}$ whose $(r+1)$ st row and $(r+1)$ st column entries are all zero except $a_{r+1, r+1}=1$, i.e.,

$$
\Xi_{\mathfrak{n}, d}^{\mathfrak{n}}=\left\{A \in \Xi_{n, d} \mid a_{r+1, j}=\delta_{r+1, j}, a_{i, r+1}=\delta_{i, r+1}, \forall i, j \in \mathbb{Z}\right\} .
$$

Introduce the following idempotent in the algebra $\mathbf{S}_{n, d}^{\mathfrak{c}}$ :

$$
\mathbf{j}_{r}=\sum_{A \in \Xi_{\mathfrak{n}, d}^{\jmath}: A \text { diagonal }}[A],
$$

and form the following subalgebra of $\mathbf{S}_{n, d}^{\mathfrak{c}}$ :

$$
\mathbf{S}_{\mathfrak{n}, d}^{\jmath}=\mathbf{j}_{r} \mathbf{S}_{n, d}^{\mathfrak{c}} \mathbf{j}_{r} .
$$

Then $\mathbf{j}_{r}$ becomes the identity of $\mathbf{S}_{\mathfrak{n}, d}^{\jmath \imath}$, which will sometimes be denoted by 1 when there is no ambiguity. Note that the algebra $\mathbf{S}_{\mathfrak{n}, d}^{j n}$ is the generic version of the convolution algebra on pairs of lattice chains in the set $\mathcal{X}_{\mathfrak{n}, d}^{j n}:=\left\{L \in \mathcal{X}_{n, d}^{\mathfrak{c}} \mid L_{r}=L_{r+1}\right\}$. The set $\left\{[A] \mid A \in \Xi_{\mathfrak{n}, d}^{\jmath n}\right\}$ forms a basis of $\mathbf{S}_{\mathfrak{n}, d}^{\jmath n}$. 
Introduce the following elements in $\mathbf{S}_{\mathfrak{n}, d}^{\jmath \imath}$ :

$$
\begin{aligned}
\check{\mathbf{e}}_{i} & =\mathbf{j}_{r} \mathbf{e}_{i} \mathbf{j}_{r}, \quad \check{\mathbf{f}}_{i}=\mathbf{j}_{r} \mathbf{f}_{i} \mathbf{j}_{r}, \\
\check{\mathbf{k}}_{i}^{ \pm 1} & =\mathbf{j}_{r} \mathbf{k}_{i}^{ \pm 1} \mathbf{j}_{r}, \quad \forall i \in[0, r-1], \\
\check{\mathbf{h}}_{a}^{ \pm 1} & =\mathbf{j}_{r} \mathbf{h}_{a}^{ \pm 1} \mathbf{j}_{r}, \quad \forall a \in[0, r], \\
\check{\mathbf{t}}_{r} & =\mathbf{j}_{r}\left(\mathbf{f}_{r} \mathbf{e}_{r}+\frac{\mathbf{k}_{r}-\mathbf{k}_{r}^{-1}}{v-v^{-1}}\right) \mathbf{j}_{r} .
\end{aligned}
$$

We note that

$$
\mathbf{j}_{r} \mathbf{e}_{r} \mathbf{j}_{r}=0, \quad \mathbf{j}_{r} \mathbf{f}_{r} \mathbf{j}_{r}=0, \quad \mathbf{j}_{r} \mathbf{e}_{r} \mathbf{f}_{r} \mathbf{j}_{r}=0 .
$$

Lusztig algebra (of type $n$ ) $\mathbf{U}_{\mathfrak{n}, d}^{\jmath \imath}$ is defined to be the subalgebra of $\mathbf{S}_{\mathfrak{n}, d}^{\jmath \imath}$ generated by the Chevalley generators $\check{\mathbf{e}}_{i}, \check{\mathbf{f}}_{i}, \check{\mathbf{k}}_{i}^{ \pm 1}$, for all $i \in[0, r-1]$, and $\check{\mathbf{t}}_{r}$.

Now let us present the type $A$ analogue of the above construction. Recall from Section 2.2 in Chapter 2 that $\Theta_{n, d}$ parametrizes a basis of $\mathbf{S}_{n, d}$. We set

$$
\Theta_{\mathfrak{n}, d}^{\jmath n}=\left\{A \in \Theta_{n, d} \mid a_{i, r+1}=0, a_{r+1, j}=0, \quad \forall i, j \in \mathbb{Z}\right\} .
$$

Similar to $\mathbf{j}_{r}$, we define the following idempotent in $\mathbf{S}_{n, d}$ :

$$
\mathbf{J}_{r}=\sum_{A \in \Theta_{\mathfrak{n}, d}^{\jmath 2}: A \text { diagonal }}[A] .
$$

As the algebra $\mathbf{J}_{r} \mathbf{S}_{n, d} \mathbf{J}_{r}$ is canonically isomorphic to $\mathbf{S}_{\mathfrak{n}, d}$ (recall $\mathfrak{n}=n-1$ ), we shall simply identify $\mathbf{S}_{\mathfrak{n}, d} \equiv \mathbf{J}_{r} \mathbf{S}_{n, d} \mathbf{J}_{r}$ below. Let

$$
\begin{aligned}
& \check{\mathbf{E}}_{i}= \begin{cases}\mathbf{J}_{r} \mathbf{E}_{i} \mathbf{J}_{r}, & \text { if } i \in[0, r-1], \\
\mathbf{J}_{r} \mathbf{E}_{r+1} \mathbf{E}_{r} \mathbf{J}_{r}, & \text { if } i=r, \\
\mathbf{J}_{r} \mathbf{E}_{i+1} \mathbf{J}_{r}, & \text { if } i \in[r+1, \mathfrak{n}-1] .\end{cases} \\
& \check{\mathbf{F}}_{i}= \begin{cases}\mathbf{J}_{r} \mathbf{F}_{i} \mathbf{J}_{r}, & \text { if } i \in[0, r-1], \\
\mathbf{J}_{r} \mathbf{F}_{r} \mathbf{F}_{r+1} \mathbf{J}_{r}, & \text { if } i=r, \\
\mathbf{J}_{r} \mathbf{F}_{i+1} \mathbf{J}_{r}, & \text { if } i \in[r+1, \mathfrak{n}-1] .\end{cases} \\
& \check{\mathbf{K}}_{i}^{ \pm 1}= \begin{cases}\mathbf{J}_{r} \mathbf{K}_{i}^{ \pm 1} \mathbf{J}_{r}, & \text { if } i \in[0, r-1], \\
\mathbf{J}_{r} \mathbf{K}_{r}^{ \pm 1} \mathbf{K}_{r+1}^{ \pm 1} \mathbf{J}_{r}, & \text { if } i=r, \\
\mathbf{J}_{r} \mathbf{K}_{i+1}^{ \pm 1} \mathbf{J}_{r}, & \text { if } i \in[r+1, \mathfrak{n}-1] .\end{cases} \\
& \check{\mathbf{H}}_{a}^{ \pm 1}= \begin{cases}\mathbf{J}_{r} \mathbf{H}_{a}^{ \pm 1} \mathbf{J}_{r}, & \text { if } a \in[0, r], \\
\mathbf{J}_{r} \mathbf{H}_{a+1}^{ \pm 1} \mathbf{J}_{r}, & \text { if } a \in[r+1, \mathfrak{n}] .\end{cases}
\end{aligned}
$$

For convenience, one can extend the range of index $i$ from the interval $[0, \mathfrak{n}-1]$ to $\mathbb{Z}$ by setting $\check{\mathbf{E}}_{i}=\check{\mathbf{E}}_{i+\mathfrak{n}}$ for all $i \in \mathbb{Z}$, etc. We shall identify $\mathbf{U}_{\mathfrak{n}, d}$ with the subalgebra of $\mathbf{S}_{\mathfrak{n}, d}$ generated by $\check{\mathbf{E}}_{i}, \check{\mathbf{F}}_{i}$ and $\check{\mathbf{K}}_{i}^{ \pm 1}$ for all $i \in[0, \mathfrak{n}-1]$.

\subsection{The comultiplication}

Recall the algebra homomorphism $\widetilde{\Delta}^{\mathfrak{c}}: \mathbf{S}_{n, d}^{\mathfrak{c}} \rightarrow \mathbf{S}_{n, d^{\prime}}^{\mathfrak{c}} \otimes \mathbf{S}_{n, d^{\prime \prime}}$ from (15.2.1), for $d^{\prime}, d^{\prime \prime}$ such that $d=d^{\prime}+d^{\prime \prime}$. We shall show its restriction to the subalgebra $\mathbf{U}_{\mathfrak{n}, d}^{\jmath}$ (denoted by the same notation) relates to the constructions above in Section 7.1 . 
Lemma 7.2.1. We have an algebra homomorphism $\widetilde{\Delta}^{\mathfrak{c}}: \mathbf{U}_{\mathfrak{n}, d}^{\jmath \imath} \longrightarrow \mathbf{U}_{\mathfrak{n}, d^{\prime}}^{\jmath \imath} \otimes \mathbf{U}_{\mathfrak{n}, d^{\prime \prime}}$. More explicitly, for $i \in[0, r-1]$, we have

$$
\begin{aligned}
\widetilde{\Delta}^{\mathfrak{c}}\left(\check{\mathbf{e}}_{i}\right) & =\check{\mathbf{\mathbf { e }}}_{i}^{\prime} \otimes \check{\mathbf{H}}_{i+1}^{\prime \prime} \check{\mathbf{H}}_{\mathfrak{n}-1-i}^{\prime \prime-1}+\check{\mathbf{h}}_{i+1}^{\prime-1} \otimes \check{\mathbf{E}}_{i}^{\prime \prime} \check{\mathbf{H}}_{\mathfrak{n}-1-i}^{\prime \prime-1}+\check{\mathbf{h}}_{i+1}^{\prime} \otimes \check{\mathbf{F}}_{\mathfrak{n}-1-i}^{\prime \prime} \check{\mathbf{H}}_{i+1}^{\prime \prime} \\
\widetilde{\Delta}^{\mathfrak{c}}\left(\check{\mathbf{f}}_{i}\right) & =\check{\mathbf{f}}_{i}^{\prime} \otimes \check{\mathbf{H}}_{i}^{\prime \prime-1} \check{\mathbf{H}}_{\mathfrak{n}-i}^{\prime \prime}+\check{\mathbf{h}}_{i}^{\prime} \otimes \check{\mathbf{F}}_{i}^{\prime \prime} \check{\mathbf{H}}_{\mathfrak{n}-1-i}^{\prime \prime}+\check{\mathbf{h}}_{i}^{\prime-1} \otimes \check{\mathbf{E}}_{\mathfrak{n}-1-i}^{\prime \prime} \check{\mathbf{H}}_{i}^{\prime \prime-1} \\
\widetilde{\Delta}^{\mathfrak{c}}\left(\check{\mathbf{k}}_{i}\right) & =\check{\mathbf{k}}_{i}^{\prime} \otimes \check{\mathbf{K}}_{i}^{\prime \prime} \check{\mathbf{K}}_{\mathfrak{n}-1-i}^{\prime \prime-1} . \\
\widetilde{\Delta}^{\mathfrak{c}}\left(\check{\mathbf{t}}_{r}\right) & =\check{\mathbf{t}}_{r}^{\prime} \otimes \check{\mathbf{K}}_{r}^{\prime \prime}+v^{2} \check{\mathbf{k}}_{r}^{\prime-1} \otimes \check{\mathbf{H}}_{r+1}^{\prime \prime} \check{\mathbf{F}}_{r}^{\prime \prime}+v^{-2} \check{\mathbf{k}}_{r}^{\prime} \otimes \check{\mathbf{H}}_{r}^{\prime \prime-1} \check{\mathbf{E}}_{r}^{\prime \prime}
\end{aligned}
$$

Proof. The fact $\widetilde{\Delta}^{\mathfrak{c}}\left(\mathbf{U}_{\mathfrak{n}, d}^{\jmath \imath}\right) \subseteq \mathbf{U}_{\mathfrak{n}, d^{\prime}}^{\jmath} \otimes \mathbf{U}_{\mathfrak{n}, d^{\prime \prime}}$ follows once we establish these explicit formulas.

We observe that $\widetilde{\Delta}^{\mathfrak{c}}\left(\mathbf{j}_{r}\right)=\mathbf{j}_{r}^{\prime} \otimes \mathbf{J}_{r}^{\prime \prime}$. So, by Proposition 5.2 .3 ,

$$
\begin{array}{r}
\widetilde{\Delta}^{\mathfrak{c}}\left(\check{\mathbf{e}}_{i}\right)=\mathbf{j}_{r}^{\prime} \otimes \mathbf{J}_{r}^{\prime \prime}\left(\mathbf{e}_{i}^{\prime} \otimes \mathbf{H}_{i+1}^{\prime \prime} \mathbf{H}_{n-1-i}^{\prime \prime-1}+\mathbf{h}_{i+1}^{\prime-1} \otimes \mathbf{E}_{i}^{\prime \prime} \mathbf{H}_{n-1-i}^{\prime \prime-1}+\mathbf{h}_{i+1}^{\prime} \otimes \mathbf{F}_{n-1-i}^{\prime \prime} \mathbf{H}_{i+1}^{\prime \prime}\right) \mathbf{j}_{r}^{\prime} \otimes \mathbf{J}_{r}^{\prime \prime} \\
=\check{\mathbf{e}}_{i}^{\prime} \otimes \check{\mathbf{H}}_{i+1}^{\prime \prime} \check{\mathbf{H}}_{\mathfrak{n}-1-i}^{\prime \prime-1}+\check{\mathbf{h}}_{i+1}^{\prime-1} \otimes \check{\mathbf{E}}_{i}^{\prime \prime} \check{\mathbf{H}}_{\mathfrak{n}-1-i}^{\prime \prime-1}+\check{\mathbf{h}}_{i+1}^{\prime} \otimes \check{\mathbf{F}}_{\mathfrak{n}-1-i}^{\prime \prime} \check{\mathbf{H}}_{i+1}^{\prime \prime}
\end{array}
$$

The formulas for $\widetilde{\Delta}^{\mathfrak{c}}\left(\check{\mathbf{f}}_{i}\right)$ and $\widetilde{\Delta}^{\mathfrak{c}}\left(\check{\mathbf{k}}_{i}\right)$ are similarly proved. The last formula can be proved in exactly the same manner as that of [FL15, Lemma 5.1.1].

Following the definition of $\phi_{d, d-n}^{\mathfrak{c}}$ in (6.1.1), we define the transfer map

$$
\phi_{d, d-\mathfrak{n}}^{\mathfrak{\jmath}}: \mathbf{S}_{\mathfrak{n}, d}^{\jmath \imath} \longrightarrow \mathbf{S}_{\mathfrak{n}, d-\mathfrak{n}}^{\jmath \imath}
$$

to be the composition

$$
\phi_{d, d-\mathfrak{n}}^{\mathfrak{\jmath}}: \mathbf{S}_{\mathfrak{n}, d}^{\jmath \imath} \stackrel{\widetilde{\Delta}^{\mathfrak{c}}}{\longrightarrow} \mathbf{S}_{\mathfrak{n}, d-\mathfrak{n}}^{\jmath \imath} \otimes \mathbf{S}_{\mathfrak{n}, \mathfrak{n}} \stackrel{1 \otimes \chi_{\mathfrak{n}}}{\longrightarrow} \mathbf{S}_{\mathfrak{n}, d-\mathfrak{n}}^{\jmath \imath}
$$

where the homomorphism $\chi_{\mathfrak{n}}: \mathbf{S}_{\mathfrak{n}, \mathfrak{n}} \longrightarrow \mathbb{Q}(v)$ is the generalized signed representation of $\mathbf{S}_{\mathfrak{n}, \mathfrak{n}}$. We have $\chi_{\mathfrak{n}}\left(\check{\mathbf{E}}_{i}\right)=\chi_{\mathfrak{n}}\left(\check{\mathbf{F}}_{i}\right)=0$ and $\chi_{\mathfrak{n}}\left(\check{\mathbf{H}}_{a}\right)=v$ for all $i \in[0, \mathfrak{n}-1], a \in[0, \mathfrak{n}]$. Thus by Lemma 7.2.1, we have for all $i \in[0, r-1]$,

$$
\phi_{d, d-\mathfrak{n}}^{\jmath \imath}\left(\check{\mathbf{e}}_{i}\right)=\check{\mathbf{e}}_{i}^{\prime}, \quad \phi_{d, d-\mathfrak{n}}^{\jmath \imath}\left(\check{\mathbf{f}}_{i}\right)=\check{\mathbf{f}}_{i}^{\prime}, \quad \phi_{d, d-\mathfrak{n}}^{\jmath \imath}\left(\check{\mathbf{k}}_{i}\right)=\check{\mathbf{k}}_{i}^{\prime}, \quad \phi_{d, d-\mathfrak{n}}^{\jmath \imath}\left(\check{\mathbf{t}}_{r}\right)=\check{\mathbf{t}}_{r}^{\prime} .
$$

Recall $\Delta^{\mathfrak{c}}: \mathbf{S}_{n, d}^{\mathfrak{c}} \longrightarrow \mathbf{S}_{n, d^{\prime}}^{\mathfrak{c}} \otimes \mathbf{S}_{n, d^{\prime \prime}}$ from (5.3.6). Let us consider the restriction $\left.\Delta^{\mathfrak{c}}\right|_{\mathbf{S}_{\mathfrak{n}, d}^{j 2}}$, which will be denoted by $\Delta^{u}$.

Proposition 7.2.2. We have an algebra homomorphism $\Delta^{\jmath \imath}: \mathbf{S}_{\mathfrak{n}, d} \longrightarrow \mathbf{S}_{\mathfrak{n}, d^{\prime}} \otimes \mathbf{S}_{\mathfrak{n}, d^{\prime \prime}}$, and by

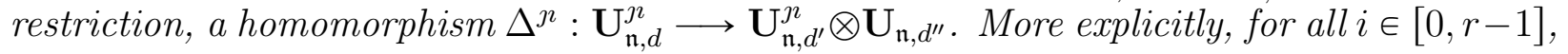
we have

$$
\begin{aligned}
\Delta^{\jmath}\left(\check{\mathbf{e}}_{i}\right) & =\check{\mathbf{e}}_{i}^{\prime} \otimes \check{\mathbf{K}}_{i}^{\prime \prime}+1 \otimes \check{\mathbf{E}}_{i}^{\prime \prime}+\check{\mathbf{k}}_{i}^{\prime} \otimes v^{\delta_{i, 0}} \check{\mathbf{F}}_{\mathfrak{n}-1-i}^{\prime \prime} \check{\mathbf{K}}_{i}^{\prime \prime} \\
\Delta^{\jmath}\left(\check{\mathbf{f}}_{i}\right) & =\check{\mathbf{f}}_{i}^{\prime} \otimes \check{\mathbf{K}}_{\mathfrak{n}-1-i}^{\prime \prime}+\check{\mathbf{k}}_{i}^{\prime-1} \otimes v^{-\delta_{i, 0}} \check{\mathbf{K}}_{\mathfrak{n}-1-i}^{\prime \prime} \check{\mathbf{F}}_{i}^{\prime \prime}+1 \otimes \check{\mathbf{E}}_{\mathfrak{n}-1-i}^{\prime \prime} \\
\Delta^{\jmath}\left(\check{\mathbf{k}}_{i}\right) & =\check{\mathbf{k}}_{i}^{\prime} \otimes \check{\mathbf{K}}_{i}^{\prime \prime} \check{\mathbf{K}}_{\mathfrak{n}-1-i}^{\prime \prime-1}, \\
\Delta^{\jmath}\left(\check{\mathbf{t}}_{r}\right) & =\check{\mathbf{t}}_{r}^{\prime} \otimes \check{\mathbf{K}}_{r}^{\prime \prime}+1 \otimes \check{\mathbf{E}}_{r}^{\prime \prime}+1 \otimes v \check{\mathbf{K}}_{r}^{\prime \prime} \check{\mathbf{F}}_{r}^{\prime \prime}
\end{aligned}
$$

Proof. Since $\Delta^{\jmath}\left(\mathbf{j}_{r}\right)=\mathbf{j}_{r}^{\prime} \otimes \mathbf{J}_{r}^{\prime \prime}$, we see that $\Delta^{\jmath}\left(\mathbf{S}_{\mathfrak{n}, d}^{\jmath \imath}\right) \subseteq \mathbf{S}_{\mathfrak{n}, d^{\prime}}^{\jmath \imath} \otimes \mathbf{S}_{\mathfrak{n}, d^{\prime \prime}}$.

So it remains to establish the formulas. The first three follow by $\Delta^{\jmath}\left(\mathbf{j}_{r}\right)=\mathbf{j}_{r}^{\prime} \otimes \mathbf{J}_{r}^{\prime \prime}$ and Proposition 5.3.4. We now prove the last one on $\Delta^{n}\left(\check{\mathbf{t}}_{r}\right)$. The superscripts ' and "are dropped for simplicity for the rest of the proof. 
By applying Proposition 5.3.4 and using that $\mathbf{j}_{r} \mathbf{e}_{r} \mathbf{j}_{r}=0$ and $\mathbf{j}_{r} \mathbf{f}_{r} \mathbf{j}_{r}=0$, we have

$$
\begin{aligned}
& \Delta^{\jmath}\left(\mathbf{j}_{r} \mathbf{f}_{r} \mathbf{e}_{r} \mathbf{j}_{r}\right)=\mathbf{j}_{r} \mathbf{f}_{r} \mathbf{e}_{r} \mathbf{j}_{r} \otimes \mathbf{J}_{r} \mathbf{K}_{r+1} \mathbf{K}_{r} \mathbf{J}_{r}+\mathbf{j}_{r} \mathbf{k}_{r}^{-1} \mathbf{j}_{r} \otimes \mathbf{J}_{r} \mathbf{K}_{r+1} \mathbf{F}_{r} \mathbf{E}_{r} \mathbf{J}_{r} \\
& +1 \otimes \mathbf{J}_{r} \mathbf{E}_{r+1} \mathbf{E}_{r} \mathbf{J}_{r}+1 \otimes \mathbf{J}_{r} \mathbf{K}_{r+1} \mathbf{F}_{r} \mathbf{F}_{r+1} \mathbf{K}_{r} \mathbf{J}_{r}+\mathbf{j}_{r} \mathbf{k}_{r} \mathbf{j}_{r} \otimes \mathbf{J}_{r} \mathbf{E}_{r+1} \mathbf{F}_{r+1} \mathbf{K}_{r} \mathbf{J}_{r} .
\end{aligned}
$$

By using the fact that

$$
\mathbf{J}_{r} \mathbf{K}_{r} \mathbf{J}_{r}=\check{\mathbf{H}}_{r}^{-1}, \mathbf{J}_{r} \mathbf{K}_{r+1} \mathbf{J}_{r}=\check{\mathbf{H}}_{r+1}, \mathbf{J}_{r} \mathbf{E}_{r} \mathbf{F}_{r} \mathbf{J}_{r}=0, \mathbf{J}_{r} \mathbf{F}_{r+1} \mathbf{E}_{r+1} \mathbf{J}_{r}=0,
$$

we have

$$
\begin{aligned}
& \mathbf{J}_{r} \mathbf{K}_{r+1} \mathbf{F}_{r} \mathbf{E}_{r} \mathbf{J}_{r}=\check{\mathbf{H}}_{r+1} \mathbf{J}_{r}\left(\mathbf{E}_{r} \mathbf{F}_{r}-\frac{\mathbf{K}_{r}-\mathbf{K}_{r}^{-1}}{v-v^{-1}}\right) \mathbf{J}_{r}=\check{\mathbf{H}}_{r+1} \frac{\check{\mathbf{H}}_{r}-\check{\mathbf{H}}_{r}^{-1}}{v-v^{-1}} \\
& \mathbf{J}_{r} \mathbf{K}_{r+1} \mathbf{F}_{r} \mathbf{F}_{r+1} \mathbf{K}_{r} \mathbf{J}_{r}=\check{\mathbf{H}}_{r+1} \check{\mathbf{F}}_{r} \check{\mathbf{H}}_{r}^{-1}=v \check{\mathbf{K}}_{r} \check{\mathbf{F}}_{r}, \\
& \mathbf{J}_{r} \mathbf{E}_{r+1} \mathbf{F}_{r+1} \mathbf{K}_{r} \mathbf{J}_{r}=\mathbf{J}_{r}\left(\mathbf{F}_{r+1} \mathbf{E}_{r+1}+\frac{\mathbf{K}_{r+1}-\mathbf{K}_{r+1}^{-1}}{v-v^{-1}}\right) \mathbf{J}_{r} \check{\mathbf{H}}_{r}^{-1}=\frac{\check{\mathbf{H}}_{r+1}-\check{\mathbf{H}}_{r+1}^{-1} \check{\mathbf{H}}_{r}^{-1} .}{v-v^{-1}}
\end{aligned}
$$

So we can rewrite (17.2.3) as follows:

$$
\begin{aligned}
\Delta^{\jmath}\left(\mathbf{j}_{r} \mathbf{f}_{r} \mathbf{e}_{r} \mathbf{j}_{r}\right) & =\mathbf{j}_{r} \mathbf{f}_{r} \mathbf{e}_{r} \mathbf{j}_{r} \otimes \check{\mathbf{K}}_{r}+1 \otimes \check{\mathbf{E}}_{r}+1 \otimes v \check{\mathbf{K}}_{r} \check{\mathbf{F}}_{r} \\
& +\mathbf{j}_{r} \mathbf{k}_{r}^{-1} \mathbf{j}_{r} \otimes \check{\mathbf{H}}_{r+1} \frac{\check{\mathbf{H}}_{r}-\check{\mathbf{H}}_{r}^{-1}}{v-v^{-1}}+\mathbf{j}_{r} \mathbf{k}_{r} \mathbf{j}_{r} \otimes \frac{\check{\mathbf{H}}_{r+1}-\check{\mathbf{H}}_{r+1}^{-1}}{v-\check{\mathbf{H}}_{r}^{-1}} \\
= & \check{\mathbf{t}}_{r} \otimes \check{\mathbf{K}}_{r}+1 \otimes \check{\mathbf{E}}_{r}+1 \otimes v \check{\mathbf{K}}_{r} \check{\mathbf{F}}_{r}+\mathbf{j}_{r} \mathbf{k}_{r}^{-1} \mathbf{j}_{r} \otimes \frac{\check{\mathbf{H}}_{r} \check{\mathbf{H}}_{r+1}}{v-v^{-1}}-\mathbf{j}_{r} \mathbf{k}_{r} \mathbf{j}_{r} \otimes \frac{\check{\mathbf{H}}_{r}^{-1} \check{\mathbf{H}}_{r+1}^{-1}}{v-v^{-1}}
\end{aligned}
$$

Finally, we have

$$
\Delta^{\jmath}\left(\mathbf{j}_{r} \frac{\mathbf{k}_{r}-\mathbf{k}_{r}^{-1}}{v-v^{-1}} \mathbf{j}_{r}\right)=\mathbf{j}_{r} \mathbf{k}_{r} \mathbf{j}_{r} \otimes \frac{\check{\mathbf{H}}_{r}^{-1} \check{\mathbf{H}}_{r+1}^{-1}}{v-v^{-1}}-\mathbf{j}_{r} \mathbf{k}_{r}^{-1} \mathbf{j}_{r} \otimes \frac{\check{\mathbf{H}}_{r} \check{\mathbf{H}}_{r+1}}{v-v^{-1}} .
$$

The formula for $\Delta^{\jmath}\left(\check{\mathbf{t}}_{r}\right)$ follows by adding the above two equations.

Now set $d^{\prime}=0$. Since $\mathbf{S}_{\mathfrak{n}, 0}^{\jmath \imath}=\mathbb{Q}(v)$, we obtain an algebra homomorphism

$$
n_{\mathfrak{n}, d}:=\left.\Delta^{\jmath \imath}\right|_{d^{\prime}=0}: \mathbf{S}_{\mathfrak{n}, d}^{\jmath \imath} \longrightarrow \mathbf{S}_{\mathfrak{n}, d},
$$

which is injective by Proposition 5.3.6. Moreover,

$$
\check{\mathbf{e}}_{i}=\check{\mathbf{f}}_{i}=0, \check{\mathbf{k}}_{i}=v^{-\delta_{i, 0}}, \check{\mathbf{t}}_{r}=1 \in \mathbf{S}_{\mathfrak{n}, 0}^{\jmath \imath}, \quad \forall i \in[0, r-1] .
$$

The following can now be read off from Proposition 7.2.2, while the injectivity of $\mu_{\mathfrak{n}, d}$ follows from a similar argument in [FL15].

Proposition 7.2.3. We have an imbedding of algebras

$$
n_{\mathfrak{n}, d}: \mathbf{S}_{\mathfrak{n}, d}^{\jmath \imath} \longrightarrow \mathbf{S}_{\mathfrak{n}, d}
$$

Moreover, for all $i \in[0, r-1]$, we have

$$
\begin{array}{cl}
n_{\mathfrak{n}, d}\left(\check{\mathbf{e}}_{i}\right)=\check{\mathbf{E}}_{i}+v^{-\delta_{i, 0}} \check{\mathbf{K}}_{i} \check{\mathbf{F}}_{\mathfrak{n}-1-i}, & n_{\mathfrak{n}, d}\left(\check{\mathbf{f}}_{i}\right)=\check{\mathbf{E}}_{\mathfrak{n}-1-i}+v^{\delta_{i, 0}} \check{\mathbf{F}}_{i} \check{\mathbf{K}}_{\mathfrak{n}-1-i}, \\
n_{\mathfrak{n}, d}\left(\check{\mathbf{k}}_{i}\right)=v^{-\delta_{i, 0}} \check{\mathbf{K}}_{i} \check{\mathbf{K}}_{\mathfrak{n}-1-i}^{-1}, & n_{\mathfrak{n}, d}\left(\check{\mathbf{t}}_{r}\right)=\check{\mathbf{E}}_{r}+v \check{\mathbf{K}}_{r} \check{\mathbf{F}}_{r}+\check{\mathbf{K}}_{r} .
\end{array}
$$

In particular, we have an imbedding of algebras $n_{\mathfrak{n}, d}: \mathbf{U}_{\mathfrak{n}, d}^{\jmath n} \rightarrow \mathbf{U}_{\mathfrak{n}, d}$. 


\subsection{The MONOMial BASIS OF $\mathbf{U}_{\mathfrak{n}, d}^{\jmath}$}

Next, we shall construct a $\jmath \imath$-monomial basis of $\mathbf{U}_{\mathfrak{n}, d}^{\jmath \imath}$, which is bar invariant and preserved by $\phi_{d, d-\mathfrak{n}}^{\mathfrak{n}}$. The compatibility of a monomial basis with $\phi_{d, d-\mathfrak{n}}^{\mathfrak{n}}$ requires additional work in the current $\imath$ setting than the previous c-case (compare Theorem 5.4.3), and this will be carried out by a similar procedure as in finite type $\imath$-version in [LW15].

Let $A$ be a matrix in $\Xi_{\mathfrak{n}, d}^{\jmath 2}$. Let $\operatorname{dlt}_{i}(A)$ be the $\mathbb{Z} \times \mathbb{Z}$ matrix obtained from $A$ by deleting the $k$ th rows and columns for all $k \equiv i \bmod n$. Note that $\operatorname{dlt}_{r+1}(A)$ and $A$ share the same $[-r, r] \times[-r, r]$-minors. The resulting matrix $\mathrm{dlt}_{r+1}(A)=\left(b_{i j}\right)$ satisfies

$$
b_{-i,-j}=b_{i j}=b_{i+\mathfrak{n}, j+\mathfrak{n}}, \sum_{i \in[1, \mathfrak{n}], j \in \mathbb{Z}} b_{i j}=2 d+1, b_{00} \in 2 \mathbb{Z}+1 .
$$

We shall denote by

$$
\check{\Xi}_{\mathfrak{n}, d}^{\jmath \imath}=\left\{B=\left(b_{i j}\right) \in \operatorname{Mat}_{\mathbb{Z} \times \mathbb{Z}}(\mathbb{N}) \mid B \text { satisfies (7.3.1) }\right\} .
$$

In particular, we have a bijection

$$
\operatorname{dlt}_{r+1}: \Xi_{\mathfrak{n}, d}^{\mathfrak{\jmath}} \longrightarrow \check{\Xi}_{\mathfrak{n}, d}^{\jmath}, \quad A \mapsto \operatorname{dlt}_{r+1}(A) .
$$

Definition 7.3.1. A matrix $A$ in $\Xi_{\mathfrak{n}, d}^{\jmath \imath}$ is called $\jmath$-aperiodic if $\operatorname{dlt}_{r+1}(A)$ is aperiodic.

Toward the construction of a suitable monomial basis, it is convenient for us to freely use parametrization of standard basis for $\mathbf{S}_{\mathfrak{n}, d}^{\jmath n}$ by matrices in $\check{\Xi}_{\mathfrak{n}, d}^{\jmath n}$ or $\Xi_{\mathfrak{n}, d}^{\jmath n}$ under such a bijection,

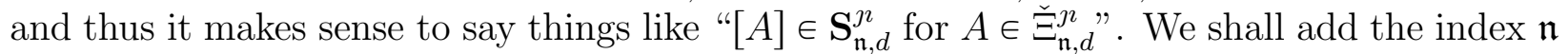
to the old notation to denote $E_{\mathfrak{n}}^{h, h+1}, E_{\theta, \mathfrak{n}}^{h, h+1}=E_{\mathfrak{n}}^{h, h+1}+E_{\mathfrak{n}}^{-h,-(h+1)}$ corresponding to $E^{h, h+1}$, $E_{\theta}^{h, h+1}$, and so on, under the bijection. (Note that the former has period $\mathfrak{n}$, while the latter has period $n$.)

Lemma 7.3.2. Let $A, B, C \in \check{\Xi}_{\mathfrak{n}, d}^{\jmath}$. Let $R$ be a positive integer.

(1) Assume that $B-R E_{\theta, \mathfrak{n}}^{h, h+1}$ is diagonal for some $h \in[0, r]$ and $\operatorname{co}(B)=\operatorname{ro}(A)$. Assume further that $R=R_{0}+\cdots+R_{l}$ and the matrix $A$ satisfy one of the following conditions:

$$
\begin{cases}a_{0 m}=0, a_{1, k+i}=R_{i}, a_{1 k} \geqslant R_{0}, a_{1 j}=0, & \text { if } h=0, k \geqslant 1 ; \\ a_{h m}=0, a_{h+1, k+i}=R_{i}, a_{h+1, k} \geqslant R_{0}, a_{h+1, j}=0, & \text { if } h \in[1, r-1] ; \\ a_{r m}=0, a_{r+1, k+i}=R_{i}, a_{r+1, k} \geqslant 2 R_{0}, a_{r+1, j}=0, & \text { if } h=r, k \geqslant r+1 ;\end{cases}
$$

for all $m \geqslant k, i \in[1, l]$ and $j>k+l$. Then we have

$$
[B] *[A]=\left[A+\sum_{i=0}^{l} R_{i}\left(E_{\theta, \mathfrak{n}}^{h, k+i}-E_{\theta, \mathfrak{n}}^{h+1, k+i}\right)\right]+\text { lower terms. }
$$

(2) Assume that $C-R E_{\theta, \mathfrak{n}}^{h+1, h}$ is diagonal for some $h \in[0, r-1]$ and $\operatorname{co}(C)=\operatorname{ro}(A)$. Assume further that $R=R_{0}+\cdots+R_{l}$ and $A$ satisfy one of the following conditions:

$$
\begin{cases}a_{1 m}=0, a_{0, k+i}=R_{i}, a_{0, k+l} \geqslant R_{l}, a_{0 j}=0, & \text { if } h=0, k+l<0 \\ a_{1 m}=0, a_{0, k+i}=R_{i}, a_{00} \geqslant 2 R_{l}, a_{0 j}=0, & \text { if } h=0, k+l=0 \\ a_{h+1, m}=0, a_{h, k+i}=R_{i}, a_{h, k+l} \geqslant R_{l}, a_{h j}=0, & \text { if } h \in[1, r-1]\end{cases}
$$


for all $m \leqslant k+l, i \in[0, l-1]$ and $j<k$. Then we have

$$
[C] *[A]=\left[A-\sum_{i=0}^{l} R_{i}\left(E_{\theta, \mathfrak{n}}^{h, k+i}-E_{\theta, \mathfrak{n}}^{h+1, k+i}\right)\right]+\text { lower terms. }
$$

Note the above multiplication formula for $h=r$ corresponds to multiplication with the new generator $\check{\mathbf{t}}_{r}$ in $\mathbf{S}_{\mathfrak{n}, d}^{\jmath \imath}$.

Proof. All cases are directly taken from Lemma 4.4.2, except the third case in (1), which can be obtained by applying Lemma 4.4.2(1), Cases 3-4, and Lemma 4.4.2(2), Case 3.

A $\jmath$-aperiodic monomial is by definition of the form $\left[X_{1}\right] * \cdots *\left[X_{m}\right]$ in $\mathbf{S}_{\mathfrak{n}, d}^{\jmath \imath}$ where $X_{i} \in \check{\Xi}_{\mathfrak{n}, d}^{\jmath \imath}$ satisfies the conditions that either $X_{i}-R E_{\theta, \mathfrak{n}}^{h, h+1}$ for $h \in[0, r]$ or $X_{i}-R E_{\theta, \mathfrak{n}}^{h+1, h}$ for $h \in[0, r-1]$ is diagonal for each $i$. The same argument as for Proposition 2.2.3 (or Theorem 5.4.3) gives us the following.

Proposition 7.3.3. For each aperiodic matrix $A$ in $\check{\Xi}_{\mathfrak{n}, d}^{\jmath}$, there exists a jl-aperiodic monomial $y_{A}$ in $\mathbf{S}_{\mathfrak{n}, d}^{\jmath \imath}$ such that $y_{A}=[A]+$ lower terms.

We freely switch the index set for $\left\{y_{A}\right\}$ back to $A \in \Xi_{\mathfrak{n}, d}^{\jmath \imath}$ under the bijection (7.3.3). By

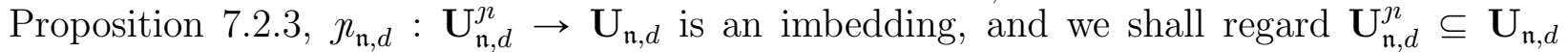
by identifying $\mathbf{U}_{\mathfrak{n}, d}^{\jmath \jmath}$ with its image under $n_{\mathfrak{n}, d}$. Recall that $\mathbf{U}_{\mathfrak{n}, d}$ admits a canonical basis $\left\{\{A\}_{d} \mid A \in \Xi_{\mathfrak{n}, d}\right.$ ll-aperiodic $\}$.

We have the following analogue of Theorem 5.4.3.

Proposition 7.3.4. The set $\left\{\{A\}_{d} \mid A \in \Xi_{\mathfrak{n}, d}^{\jmath}\right.$ l-aperiodic $\}$ forms a (canonical) basis for $\mathbf{U}_{\mathfrak{n}, d}^{\jmath \imath}$. Also, $\left\{y_{A} \mid A \in \Xi_{\mathfrak{n}, d}^{\jmath n}\right.$ l-aperiodic $\}$ forms a monomial basis for $\mathbf{U}_{\mathfrak{n}, d}^{\jmath n}$.

Proof. We have an imbedding $n_{\mathfrak{n}, d}: \mathbf{U}_{\mathfrak{n}, d}^{\jmath \imath} \rightarrow \mathbf{U}_{\mathfrak{n}, d}$ by Proposition 7.2.3, A counterpart of Proposition 5.4.2 makes sense in our setting. We also have Proposition 7.3.3. Therefore we have all the three key ingredients available to rerun the argument for Theorem 5.4.3. The proposition is proved.

Note that $y_{A}$ is not bar invariant in general. As in the finite $\imath$-setting [LW15], this monomial basis $\left\{y_{A}\right\}$ is not preserved by the transfer map $\phi_{\mathfrak{n}, \mathfrak{n}-d}^{\mathfrak{n}}$, and thus this basis is not directly applicable for studying the limiting algebra $\mathbf{U}_{\mathfrak{n}}^{\jmath \imath}$ in the following Section 7.4 . To overcome this obstacle, we introduce the hybrid monomial $h_{A}$ obtained from $y_{A}$ by replacing every factor $\left[X_{i}\right]$ in the monomial $y_{A}$ by its associated canonical basis element $\left\{X_{i}\right\}_{d}$ if $X_{i}$ is of the form $X_{i}=X(D, R):=D+R E_{\theta, \mathfrak{n}}^{r, r+1}$ for some diagonal matrix $D$ and for some $R$. We still have $\left\{X_{i}\right\}_{d} \in \mathbf{S}_{\mathfrak{n}, d}^{\jmath}$ thanks to the fact that $\left\{X_{i}\right\}_{d} \in\left[X_{i}\right]+\sum_{0 \leqslant k<R} \mathcal{A} X\left(D_{k}, k\right)$ for some diagonal matrices $D_{k}$; see [LW15]. Hence we have $h_{A}=y_{A}+$ lower terms $\in \mathbf{S}_{\mathfrak{n}, d}^{\jmath \imath}$. Thus we have obtained the following.

Proposition 7.3.5. For each aperiodic matrix $A$ in $\check{\Xi}_{\mathfrak{n}, d}^{\jmath}$, there exists a l-aperiodic hybrid monomial $h_{A}$ in $\mathbf{S}_{\mathfrak{n}, d}^{\jmath \imath}$ such that $h_{A}=[A]+$ lower terms, $\overline{h_{A}}=h_{A}$, and $\phi_{d, d-\mathfrak{n}}^{\jmath n}\left(h_{A}\right)=h_{A-2 I_{\mathfrak{n}}}$, with $I_{\mathfrak{n}}=\sum_{1 \leqslant i \leqslant \mathfrak{n}} E_{\mathfrak{n}}^{i i}$. Moreover, $\left\{h_{A} \mid A \in \check{\Xi}_{\mathfrak{n}, d}^{\jmath \mathfrak{l}}\right.$ lu-aperiodic $\}$ forms a (hybrid) monomial basis for $\mathbf{U}_{\mathfrak{n}, d}^{\mathfrak{l}}$.

(It is understood above that $h_{A-2 I_{\mathfrak{n}}}=0$ if $A-2 I_{\mathfrak{n}}$ contains some negative entry.) 
Example 7.3.6. Set $r=2$ and so $\mathfrak{n}=5$. Consider the $\jmath$-aperiodic matrix $A \in \check{\Xi}_{5, d}^{\jmath \imath}$ :

$A=$\begin{tabular}{c|c|c|c|c|c|c|c|c|c|c|c} 
& $\mathrm{c}-3$ & $\mathrm{c}-2$ & $\mathrm{c}-1$ & $\mathrm{c} 0$ & $\mathrm{c} 1$ & $\mathrm{c} 2$ & $\mathrm{c} 3$ & $\mathrm{c} 4$ & $\mathrm{c} 5$ & $\mathrm{c} 6$ & $\mathrm{c} 7$ \\
\hline & & & & & & & & & & & \\
\hline $\mathrm{r} 0$ & & 3 & 1 & $*$ & 1 & 3 & & & & & \\
\hline $\mathrm{r} 1$ & & & 0 & 6 & $*$ & 0 & 4 & & & & \\
\hline $\mathrm{r} 2$ & & & & 8 & 7 & $*$ & 2 & 5 & & & \\
\hline $\mathrm{r} 3$ & & & & & 5 & 2 & $*$ & 7 & 8 & & \\
\hline $\mathrm{r} 4$ & & & & & & 4 & 0 & $*$ & 6 & 0 & \\
\hline $\mathrm{r} 5$ & & & & & & & 3 & 1 & $*$ & 1 & 3 \\
\hline & & & & & & & & & & &
\end{tabular}

where 'ri' and 'cj' in the table indicate the $i$-th row and $j$-th column of the matrix $A$, respectively. We have

$$
\begin{aligned}
y_{A} & =\check{\mathbf{e}}_{1}^{(8)} * \check{\mathbf{t}}_{2}^{[5]} * \check{\mathbf{f}}_{1}^{(4)} * \check{\mathbf{f}}_{0}^{(4)} * \check{\mathbf{e}}_{0}^{(14)} * \check{\mathbf{e}}_{1}^{(12)} * \check{\mathbf{t}}_{2}^{[6]} * \check{\mathbf{f}}_{0}^{(3)} * 1_{\operatorname{co}(A)}=[A]+\text { lower terms, } \\
h_{A} & =\check{\mathbf{e}}_{1}^{(8)} * \check{\mathbf{t}}_{2}^{\{5\}} * \check{\mathbf{f}}_{1}^{(4)} * \check{\mathbf{f}}_{0}^{(4)} * \check{\mathbf{e}}_{0}^{(14)} * \check{\mathbf{e}}_{1}^{(12)} * \check{\mathbf{t}}_{2}^{\{6\}} * \check{\mathbf{f}}_{0}^{(3)} * 1_{\operatorname{co}(A)}=[A]+\text { lower terms, }
\end{aligned}
$$

where $\check{\mathbf{t}}_{2}^{[R]}$ and $\check{\mathbf{t}}_{2}^{\{R\}}$ denote $\sum_{X}[X]$ and $\sum_{X}\{X\}_{d}$, respectively, with the sum taken over $X$ such that $X-R E_{\theta, \mathfrak{n}}^{r, r+1}$ is diagonal.

\subsection{The Coideal SubalgeBra of TYPE $\jmath$}

Now that the results at the $\jmath$-Schur algebra level are established (which is the counterpart of Chapter 5), we will formulate the $\imath$-analogue of Chapter 6. As most of these are straightforward, we will skip some of the details.

Starting with the projective system $\left\{\left(\mathbf{U}_{\mathfrak{n}, d}^{\jmath n}, \phi_{d, d-\mathfrak{n}}^{\mathfrak{n}}\right)\right\}_{d \in \mathbb{N}}$, we construct two distinguished algebras $\mathbf{U}_{\mathfrak{n}}^{\jmath \imath}$ and $\dot{\mathbf{U}}_{\mathfrak{n}}^{\jmath \imath}$ out of the associated projective limit algebra $\mathbf{U}_{\mathfrak{n}, \infty}^{\jmath \imath}$; the Chevalley generators of $\mathbf{U}_{\mathfrak{n}}^{\jmath n}$ will be denoted again by $\check{\mathbf{e}}_{i}, \check{\mathbf{f}}_{i}, \check{\mathbf{k}}_{i}^{ \pm 1}(i \in[0, r-1])$, and $\check{\mathbf{t}}_{r}$. The family of imbeddings $\left\{n_{\mathfrak{n}, d}: \mathbf{U}_{\mathfrak{n}, d}^{\mathfrak{n}} \rightarrow \mathbf{U}_{\mathfrak{n}, d}\right\}_{d \geqslant 0}$ induces an algebra imbedding $n_{\mathfrak{n}}: \mathbf{U}_{\mathfrak{n}}^{\mathfrak{n}} \rightarrow \mathbf{U}_{\mathfrak{n}}$. The family of $\Delta^{u}$ (for various $d^{\prime}+d^{\prime \prime}=d$ ) induce an algebra homomorphism (which is coassociative in a suitable sense) $\Delta^{\jmath}: \mathbf{U}_{\mathfrak{n}}^{\jmath \imath} \rightarrow \mathbf{U}_{\mathfrak{n}}^{\jmath \imath} \otimes \mathbf{U}_{\mathfrak{n}}$, whose action on the Chevalley generators can be presented explicitly. Recall the algebra isomorphism $\mathbf{U}_{\mathfrak{n}} \cong \mathbf{U}\left(\widehat{\mathfrak{s l}}_{\mathfrak{n}}\right)$. Summarizing we have established the following.

Theorem 7.4.1. The pair $\left(\mathbf{U}\left(\widehat{\mathfrak{s l}}_{\mathfrak{n}}\right), \mathbf{U}_{\mathfrak{n}}^{\jmath n}\right)$ forms an affine quantum symmetric pair. (see Figure 2 for the relevant involution.)

Recall the Cartan integers $c_{i j}$ from (5.1.7). We give a presentation for the algebra $\mathbf{U}_{\mathfrak{n}}^{\jmath \imath}$, which is a counterpart of Proposition 6.1.5 for $\mathbf{U}_{n}^{\mathfrak{c}}$. This presentation is a variant of [Ko14, Theorem 7.1] in our setting and our notation. Recall we always assume $r \geqslant 1$ so $\mathfrak{n} \geqslant 3$. 
Proposition 7.4.2. The $\mathbb{Q}(v)$-algebra $\mathbf{U}_{\mathfrak{n}}^{\jmath n}$ has a presentation with generators $\check{\mathbf{e}}_{i}, \check{\mathbf{f}}_{i}$, and $\check{\mathbf{k}}_{i}^{ \pm 1}$ $(i \in[0, r-1])$ and $\check{\mathbf{t}}_{r}$, and the following relations: for all $i, j \in[0, r-1]$,

$$
\begin{aligned}
& \check{\mathbf{k}}_{0}\left(\check{\mathbf{k}}_{1}^{2} \cdots \check{\mathbf{k}}_{r-1}^{2}\right)=v^{-1} \\
& \check{\mathbf{k}}_{i} \check{\mathbf{k}}_{i}^{-1}=1, \quad \check{\mathbf{k}}_{i} \check{\mathbf{k}}_{j}=\check{\mathbf{k}}_{j} \check{\mathbf{k}}_{i}, \quad \check{\mathbf{k}} \check{\mathbf{t}}_{r}=\check{\mathbf{t}} \check{\mathbf{k}}_{r}, \\
& \check{\mathbf{k}}_{i} \check{\mathbf{e}}_{j} \check{\mathbf{k}}_{i}^{-1}=v^{c_{i j}+\delta_{i, 0} \delta_{j, 0}} \check{\mathbf{e}}_{j} \text {, } \\
& \check{\mathbf{k}}_{i} \check{\mathbf{f}}_{j} \check{\mathbf{k}}_{i}^{-1}=v^{-c_{i j}-\delta_{i, 0} \delta_{j, 0}} \check{\mathbf{f}}_{j}, \\
& \check{\mathbf{e}}_{i} \check{\mathbf{e}}_{j}=\check{\mathbf{e}}_{j} \check{\mathbf{e}}_{i}, \quad \check{\mathbf{f}}_{i} \check{\mathbf{f}}_{j}=\check{\mathbf{f}}_{j} \check{\mathbf{f}}_{i}, \quad \forall|i-j|>1, \\
& \check{\mathbf{e}}_{i} \check{\mathbf{t}}_{r}=\check{\mathbf{t}}_{r} \check{\mathbf{e}}_{i}, \quad \check{\mathbf{f}}_{i} \check{\mathbf{t}}_{r}=\check{\mathbf{t}}_{r} \check{\mathbf{f}}_{i}, \quad \forall i \leqslant r-2, \\
& \check{\mathbf{e}}_{i}^{2} \check{\mathbf{e}}_{j}+\check{\mathbf{e}}_{j} \check{\mathbf{e}}_{i}^{2}=\left(v+v^{-1}\right) \check{\mathbf{e}}_{i} \check{\mathbf{e}}_{j} \check{\mathbf{e}}_{i}, \quad \forall|i-j|=1, \\
& \check{\mathbf{f}}_{i}^{2} \check{\mathbf{f}}_{j}+\check{\mathbf{f}}_{j} \check{\mathbf{f}}_{i}^{2}=\left(v+v^{-1}\right) \check{\mathbf{f}}_{i} \check{\mathbf{f}}_{j} \check{\mathbf{f}}_{i}, \quad \forall|i-j|=1, \\
& \check{\mathbf{e}}_{r-1}^{2} \check{\mathbf{t}}_{r}+\check{\mathbf{t}}_{r} \check{\mathbf{e}}_{r-1}^{2}=\left(v+v^{-1}\right) \check{\mathbf{e}}_{r-1} \check{\mathbf{t}}_{r} \check{\mathbf{e}}_{r-1}, \\
& \check{\mathbf{f}}_{r-1}^{2} \check{\mathbf{t}}_{r}+\check{\mathbf{t}}_{r} \check{\mathbf{f}}_{r-1}^{2}=\left(v+v^{-1}\right) \check{\mathbf{f}}_{r-1} \check{\mathbf{t}}_{r} \check{\mathbf{f}}_{r-1}, \\
& \check{\mathbf{t}}_{r}^{2} \check{\mathbf{e}}_{r-1}+\check{\mathbf{e}}_{r-1} \check{\mathbf{t}}_{r}^{2}=\left(v+v^{-1}\right) \check{\mathbf{e}}_{r-1} \check{\mathbf{t}}_{r} \check{\mathbf{e}}_{r-1}+\check{\mathbf{e}}_{r-1} \text {, } \\
& \check{\mathbf{t}}_{r}^{2} \check{\mathbf{f}}_{r-1}+\check{\mathbf{f}}_{r-1} \check{\mathbf{t}}_{r}^{2}=\left(v+v^{-1}\right) \check{\mathbf{f}}_{r-1} \check{\mathbf{t}}_{r} \check{\mathbf{f}}_{r-1}+\check{\mathbf{f}}_{r-1}, \\
& \check{\mathbf{e}}_{i} \check{\mathbf{f}}_{j}-\check{\mathbf{f}}_{j} \check{\mathbf{e}}_{i}=\delta_{i j} \frac{\check{\mathbf{k}}_{i}-\check{\mathbf{k}}_{i}^{-1}}{v-v^{-1}}, \quad \forall(i, j) \neq(0,0), \\
& \check{\mathbf{e}}_{0}^{2} \check{\mathbf{f}}_{0}+\check{\mathbf{f}}_{0} \check{\mathbf{e}}_{0}^{2}=\left(v+v^{-1}\right)\left(\check{\mathbf{e}}_{0} \check{\mathbf{f}}_{0} \check{\mathbf{e}}_{0}-\left(v \check{\mathbf{k}}_{0}+v^{-1} \check{\mathbf{k}}_{0}^{-1}\right) \check{\mathbf{e}}_{0}\right), \\
& \check{\mathbf{f}}_{0}^{2} \check{\mathbf{e}}_{0}+\check{\mathbf{e}}_{0} \check{\mathbf{f}}_{0}^{2}=\left(v+v^{-1}\right)\left(\check{\mathbf{f}}_{0} \check{\mathbf{e}}_{0} \check{\mathbf{f}}_{0}-\check{\mathbf{f}}_{0}\left(v \check{\mathbf{k}}_{0}+v^{-1} \check{\mathbf{k}}_{0}^{-1}\right)\right) \text {. }
\end{aligned}
$$

Proof. We verify directly the above relations for Lusztig algebras $\mathbf{U}_{\mathfrak{n}, d}^{\jmath \imath}$, and it follows that the relations hold for $\mathbf{U}_{\mathfrak{n}}^{\jmath}$ by construction. Then we use Theorem 7.4.1 and [Ko14, Theorem 7.1] to conclude that we do not need additional relations.

Now the construction of canonical basis with positivity for the coideal algebra in Section 6.4 can be repeated. Recalling $\widetilde{\Xi}_{n}$ from $(\underline{6.2 .2})$, we introduce the following subsets of $\widetilde{\Xi}_{n}$ :

$$
\begin{aligned}
\widetilde{\Xi}_{\mathfrak{n}}^{\jmath} & =\left\{A=\left(a_{i j}\right) \in \widetilde{\Xi}_{n} \mid a_{r+1, j}=\delta_{r+1, j}, a_{i, r+1}=\delta_{i, r+1}\right\}, \\
\widetilde{\Xi}_{\mathfrak{n}}^{\jmath, a p} & =\left\{A \in \widetilde{\Xi}_{\mathfrak{n}}^{\jmath l} \mid A \text { is } \jmath \text {-aperiodic }\right\} .
\end{aligned}
$$

Recalling $\widetilde{\Xi}_{n, d}$ from (6.2.3), we further introduce, for $d \in \mathbb{Z}$,

$$
\widetilde{\Xi}_{\mathfrak{n}, d}^{\jmath n}=\left\{A \in \widetilde{\Xi}_{\mathfrak{n}}^{\jmath \imath}|| A \mid=d\right\}, \quad \widetilde{\Xi}_{\mathfrak{n}}^{\jmath \imath}=\bigcup_{d} \widetilde{\Xi}_{\mathfrak{n}, d}^{\jmath}
$$

We define an equivalence relation $\approx$ on $\widetilde{\Xi}_{\mathfrak{n}}^{\jmath, a p}$ as in (6.2.4) and let $\hat{A}$ be the equivalence class of $A$. The hybrid monomial basis $\left\{h_{A}\right\}$ for $\mathbf{S}_{\mathfrak{n}, d}^{\mathfrak{\imath}}$ (cf. Proposition 7.3.5) gives rise to a monomial basis $\left\{h_{\widehat{A}} \mid \widehat{A} \in \widetilde{\Xi}_{\mathfrak{n}}^{\jmath, a p} / \approx\right\}$ for the algebra $\dot{\mathbf{U}}_{\mathfrak{n}}^{\jmath \imath}$. A bilinear form $\langle\cdot, \cdot\rangle$ on $\dot{\mathbf{U}}_{\mathfrak{n}}^{\jmath \imath}$ can be defined similarly as in Section 6.4 and shown to be non-degenerate. The following is a $\jmath$-analogue of Theorems 6.4.3 and 6.4.5. 
Theorem 7.4.3. There exists a canonical basis $\dot{\mathbf{B}}_{\mathfrak{n}}^{\jmath n}=\left\{b_{\hat{A}} \mid \widehat{A} \in \widetilde{\Xi}_{\mathfrak{n}}^{\jmath n, a p} / \approx\right\}$ for $\dot{\mathbf{U}}_{\mathfrak{n}}^{\jmath n}$, whose transition matrix with respect to the monomial basis $\left\{h_{\hat{A}} \mid \widehat{A} \in \widetilde{\Xi}_{\mathfrak{n}}^{\jmath l, a p} / \approx\right\}$ is uni-triangular. Moreover, the structure constants of the canonical basis $\dot{\mathbf{B}}_{\mathfrak{n}}^{j n}$ lie in $\mathbb{N}\left[v, v^{-1}\right]$ with respect to the multiplication and comultiplication, and in $v^{-1} \mathbb{N}\left[\left[v^{-1}\right]\right]$ with respect to the bilinear pairing. 


\section{CHAPTER 8}

\section{More variants of coideal subalgebras of quantum affine $\mathfrak{s l}_{n}$}

This chapter offers two more variants of geometric origin (denoted by types $\vartheta$ and $\imath$ ) of the constructions in Chapters 6 and 7 . Set

$$
\eta=\mathfrak{n}-1=n-2=2 r(r \geqslant 1) .
$$

Schur algebras $\mathbf{S}_{\mathfrak{n}, d}^{\imath \jmath}$ and Lusztig algebras $\mathbf{U}_{\mathfrak{n}, d}^{\imath \jmath}$ are introduced, and the family of Lusztig algebras gives rise to algebras $\mathbf{U}_{\mathfrak{n}}^{\imath \jmath}$ and $\dot{\mathbf{U}}_{\mathfrak{n}}^{\imath \jmath}$. We show that $\left(\mathbf{U}\left(\widehat{\mathfrak{s l}}_{\mathfrak{n}}\right), \mathbf{U}_{\mathfrak{n}}^{\imath \jmath}\right)$ forms an affine quantum symmetric pair. In addition, a family of algebras $\mathbf{U}_{\eta, d}^{\imath \imath} \subset \mathbf{S}_{\eta, d}^{\imath \imath}$ is introduced and gives rise to algebras $\mathbf{U}_{\eta}^{\imath}$ and $\dot{\mathbf{U}}_{\eta}^{\imath}$. Then $\left(\mathbf{U}\left(\widehat{\mathfrak{s l}}_{\eta}\right), \mathbf{U}_{\eta}^{\imath \imath}\right)$ forms an affine quantum symmetric pair. The canonical bases of both algebras $\dot{\mathbf{U}}_{\mathfrak{n}}^{\imath \jmath}$ and $\dot{\mathbf{U}}_{\eta}^{\imath \imath}$ admit positivity with respect to multiplication, comultiplication, and a bilinear pairing.

\subsection{The Schur algebras of TyPe $\vartheta$}

Recall the set $\Xi_{n, d}$ from (4.2.1). We set

$$
\Xi_{\mathfrak{n}, d}^{\imath \jmath}=\left\{A \in \Xi_{n, d} \mid a_{0, j}=\delta_{0, j}, a_{i, 0}=\delta_{i, 0}, \forall i, j \in \mathbb{Z}\right\} .
$$

Introduce the idempotent $\mathbf{j}_{0}$ in the algebra $\mathbf{S}_{n, d}^{\mathfrak{c}}$ given by $\mathbf{j}_{0}=\sum_{A \in \Xi_{\mathfrak{n}, d}^{\imath \jmath} \text { diagonal }}[A]$, and form the following subalgebra of $\mathbf{S}_{n, d}^{\mathfrak{c}}$ (called Schur algebra of type $\eta$ ):

$$
\mathbf{S}_{\mathfrak{n}, d}^{\imath \jmath}=\mathbf{j}_{0} \mathbf{S}_{n, d}^{\mathfrak{c}} \mathbf{j}_{0} .
$$

We further introduce the following elements in $\mathbf{S}_{\mathfrak{n}, d}^{\imath \jmath}$ :

$$
\begin{aligned}
\hat{\mathbf{e}}_{i} & =\mathbf{j}_{0} \mathbf{e}_{i} \mathbf{j}_{0}, \quad \hat{\mathbf{f}}_{i}=\mathbf{j}_{0} \mathbf{f}_{i} \mathbf{j}_{0}, \\
\hat{\mathbf{k}}_{i}^{ \pm 1} & =\mathbf{j}_{0} \mathbf{k}_{i}^{ \pm 1} \mathbf{j}_{0}, \quad \forall i \in[1, r], \\
\hat{\mathbf{h}}_{a}^{ \pm 1} & =\mathbf{j}_{0} \mathbf{h}_{a}^{ \pm 1} \mathbf{j}_{0}, \quad \forall a \in[0, r], \\
\hat{\mathbf{t}}_{0} & =\mathbf{j}_{0}\left(\mathbf{e}_{0} \mathbf{f}_{0}+\frac{\mathbf{k}_{0}^{-1}-\mathbf{k}_{0}}{v-v^{-1}}\right) \mathbf{j}_{0} .
\end{aligned}
$$

We also have the following vanishing results in $\mathbf{S}_{\mathfrak{n}, d}^{\imath \jmath}$, which will be used freely:

$$
\mathbf{j}_{0} \mathbf{e}_{0} \mathbf{j}_{0}=0, \quad \mathbf{j}_{0} \mathbf{f}_{0} \mathbf{j}_{0}=0, \quad \mathbf{j}_{0} \mathbf{f}_{0} \mathbf{e}_{0} \mathbf{j}_{0}=0 .
$$

The Lusztig algebra $\mathbf{U}_{\mathfrak{n}, d}^{\imath \jmath}$ is defined to be the subalgebra of $\mathbf{S}_{\mathfrak{n}, d}^{\imath \jmath}$ generated by the Chevalley generators $\hat{\mathbf{e}}_{i}, \hat{\mathbf{f}}_{i}, \hat{\mathbf{k}}_{i}^{ \pm 1}$, for $i \in[1, r]$, and $\hat{\mathbf{t}}_{0}$.

Let us also formulate a type $A$ version which is compatible with the above construction. Let

$$
\Theta_{\mathfrak{n}, d}^{\imath \jmath}=\left\{A \in \Theta_{n, d} \mid a_{0, j}=0, a_{i, 0}=0, \quad \forall i, j \in \mathbb{Z}\right\}
$$


Using the idempotent $\mathbf{J}_{0}$ in $\mathbf{S}_{n, d}$ given by $\mathbf{J}_{0}=\sum_{A \in \Theta_{n, d}^{\imath \jmath} \text { diagonal }}[A]$, we form the subalgebra $\mathbf{J}_{0} \mathbf{S}_{n, d} \mathbf{J}_{0}$ of $\mathbf{S}_{n, d}$, which is isomorphic to the algebra $\mathbf{S}_{\mathfrak{n}, d}$ defined earlier. We shall always identify $\mathbf{S}_{\mathfrak{n}, d} \equiv \mathbf{J}_{0} \mathbf{S}_{n, d} \mathbf{J}_{0}$ below. We introduce the following elements in $\mathbf{S}_{\mathfrak{n}, d}$ :

$$
\begin{aligned}
\hat{\mathbf{E}}_{i} & = \begin{cases}\mathbf{J}_{0} \mathbf{E}_{0} \mathbf{E}_{-1} \mathbf{J}_{0}, & \text { if } i=0, \\
\mathbf{J}_{0} \mathbf{E}_{i} \mathbf{J}_{0}, & \text { if } i \in[1, \mathfrak{n}-1] .\end{cases} \\
\hat{\mathbf{F}}_{i} & = \begin{cases}\mathbf{J}_{0} \mathbf{F}_{-1} \mathbf{F}_{0} \mathbf{J}_{0}, & \text { if } i=0, \\
\mathbf{J}_{0} \mathbf{F}_{i} \mathbf{J}_{0}, & \text { if } i \in[1, \mathfrak{n}-1] .\end{cases} \\
\hat{\mathbf{K}}_{i}^{ \pm 1} & = \begin{cases}\mathbf{J}_{0} \mathbf{K}_{0}^{ \pm 1} \mathbf{K}_{-1}^{ \pm 1} \mathbf{J}_{0}, & \text { if } i=0, \\
\mathbf{J}_{0} \mathbf{K}_{i}^{ \pm 1} \mathbf{J}_{0}, & \text { if } i \in[1, \mathfrak{n}-1] .\end{cases} \\
\hat{\mathbf{H}}_{a}^{ \pm 1} & = \begin{cases}\mathbf{J}_{0} \mathbf{H}_{-1}^{ \pm 1} \mathbf{J}_{0}, & \text { if } a=0, \\
\mathbf{J}_{0} \mathbf{H}_{i}^{ \pm 1} \mathbf{J}_{0}, & \text { if } a \in[1, \mathfrak{n}] .\end{cases}
\end{aligned}
$$

We can extend the interval $i \in[0, \mathfrak{n}-1]$ to $i \in \mathbb{Z}$ by setting $\hat{\mathbf{E}}_{i}=\hat{\mathbf{E}}_{i+\mathfrak{n}}$, etc. We observe

$$
\mathbf{J}_{0} \mathbf{E}_{-1} \mathbf{J}_{0}=0, \quad \mathbf{J}_{0} \mathbf{F}_{-1} \mathbf{J}_{0}=0, \quad \mathbf{J}_{0} \mathbf{E}_{-1} \mathbf{F}_{-1} \mathbf{J}_{0}=0, \quad \mathbf{J}_{0} \mathbf{F}_{0} \mathbf{E}_{0} \mathbf{J}_{0}=0 .
$$

We identify $\mathbf{U}_{\mathfrak{n}, d}$ with the subalgebra generated by the Chevalley generators $\hat{\mathbf{E}}_{i}, \hat{\mathbf{F}}_{i}$ and $\hat{\mathbf{K}}_{i}^{ \pm 1}$ for all $i \in[0, \mathfrak{n}-1]$.

\subsection{Comultiplication And transfer map of TyPe $\vartheta$}

We shall study the restriction to Lusztig algebra $\mathbf{U}_{\mathfrak{n}, d}^{\imath \jmath}$ (denoted by the same notation) of $\widetilde{\Delta}^{\mathfrak{c}}: \mathbf{S}_{n, d}^{\mathfrak{c}} \rightarrow \mathbf{S}_{n, d^{\prime}}^{\mathfrak{c}} \otimes \mathbf{S}_{n, d^{\prime \prime}}$ from (5.2.1), for arbitrary $d^{\prime}, d^{\prime \prime}$ such that $d=d^{\prime}+d^{\prime \prime}$.

Proposition 8.2.1. We have an algebra homomorphism

$$
\widetilde{\Delta}^{\mathfrak{c}}: \mathbf{U}_{\mathfrak{n}, d}^{\imath \jmath} \longrightarrow \mathbf{U}_{\mathfrak{n}, d^{\prime}}^{\imath \jmath} \otimes \mathbf{U}_{\mathfrak{n}, d^{\prime \prime}}
$$

More explicitly, for all $i \in[1, r]$, we have

$$
\begin{aligned}
\widetilde{\Delta}^{\mathfrak{c}}\left(\hat{\mathbf{e}}_{i}\right) & =\hat{\mathbf{e}}_{i}^{\prime} \otimes \hat{\mathbf{H}}_{i+1}^{\prime \prime} \hat{\mathbf{H}}_{n-1-i}^{\prime \prime-1}+\hat{\mathbf{h}}_{i+1}^{\prime-1} \otimes \hat{\mathbf{E}}_{i}^{\prime \prime} \hat{\mathbf{H}}_{n-1-i}^{\prime \prime-1}+\hat{\mathbf{h}}_{i+1}^{\prime} \otimes \hat{\mathbf{F}}_{n-1-i}^{\prime \prime} \hat{\mathbf{H}}_{i+1}^{\prime \prime}, \\
\widetilde{\Delta}^{\mathfrak{c}}\left(\hat{\mathbf{f}}_{i}\right) & =\hat{\mathbf{f}}_{i}^{\prime} \otimes \hat{\mathbf{H}}_{i}^{\prime \prime-1} \hat{\mathbf{H}}_{n-i}^{\prime \prime}+\hat{\mathbf{h}}_{i}^{\prime} \otimes \hat{\mathbf{F}}_{i}^{\prime \prime} \hat{\mathbf{H}}_{n-i}^{\prime \prime}+\hat{\mathbf{h}}_{i}^{\prime-1} \otimes \hat{\mathbf{E}}_{n-1-i}^{\prime \prime} \hat{\mathbf{H}}_{i}^{\prime \prime-1}, \\
\widetilde{\Delta}^{\mathfrak{c}}\left(\hat{\mathbf{k}}_{i}\right) & =\hat{\mathbf{k}}_{i}^{\prime} \otimes \hat{\mathbf{K}}_{i}^{\prime \prime} \hat{\mathbf{K}}_{n-1-i}^{\prime \prime-1}, \\
\widetilde{\Delta}^{\mathfrak{c}}\left(\hat{\mathbf{t}}_{0}\right) & =\hat{\mathbf{t}}_{0}^{\prime} \otimes \hat{\mathbf{K}}_{0}^{\prime \prime}+v^{2} \hat{\mathbf{k}}_{0}^{\prime} \otimes \hat{\mathbf{H}}_{1}^{\prime \prime} \hat{\mathbf{F}}_{0}^{\prime \prime}+v^{-2} \hat{\mathbf{k}}_{0}^{\prime-1} \otimes \hat{\mathbf{H}}_{0}^{\prime \prime-1} \hat{\mathbf{E}}_{0}^{\prime \prime} .
\end{aligned}
$$

Proof. The inclusion $\widetilde{\Delta}^{\mathfrak{c}}\left(\mathbf{U}_{\mathfrak{n}, d}^{\imath \jmath}\right) \subseteq \mathbf{U}_{\mathfrak{n}, d^{\prime}}^{\imath \jmath} \otimes \mathbf{U}_{\mathfrak{n}, d^{\prime \prime}}$ follows once the formulas are established. The superscripts ' and " will be dropped throughout the proof for the sake of simplicity. The first three formulas follow from Proposition 5.2.3. To prove the last one, we proceed similarly as in the $\jmath$-version. By using $\mathbf{j}_{0} \mathbf{e}_{0} \mathbf{j}_{0}=0$ and $\mathbf{j}_{0} \mathbf{f}_{0} \mathbf{j}_{0}=0$, we have

$$
\begin{aligned}
\widetilde{\Delta}^{\mathfrak{c}}\left(\mathbf{j}_{0} \mathbf{e}_{0} \mathbf{f}_{0} \mathbf{j}_{0}\right) & =\mathbf{j}_{0} \otimes \mathbf{J}_{0} \widetilde{\Delta}^{\mathfrak{c}}\left(\mathbf{e}_{0}\right) \widetilde{\Delta}^{\mathfrak{c}}\left(\mathbf{f}_{0}\right) \mathbf{j}_{0} \otimes \mathbf{J}_{0} \\
& =\mathbf{j}_{0} \mathbf{e}_{0} \mathbf{f}_{0} \mathbf{j}_{0} \otimes \mathbf{J}_{0} \mathbf{H}_{1} \mathbf{H}_{-1}^{-1} \mathbf{J}_{0}+\mathbf{j}_{0} \mathbf{h}_{1}^{-1} \mathbf{h}_{0} \mathbf{j}_{0} \otimes \mathbf{J}_{0} \mathbf{E}_{0} \mathbf{H}_{-1}^{-1} \mathbf{F}_{0} \mathbf{H}_{0} \mathbf{J}_{0} \\
& +\mathbf{j}_{0} \mathbf{h}_{1} \mathbf{h}_{0} \mathbf{j}_{0} \otimes \mathbf{J}_{0} \mathbf{F}_{-1} \mathbf{H}_{1} \mathbf{F}_{0} \mathbf{H}_{0} \mathbf{J}_{0}+\mathbf{j}_{0} \mathbf{h}_{1}^{-1} \mathbf{h}_{0}^{-1} \mathbf{j}_{0} \otimes \mathbf{J}_{0} \mathbf{E}_{0} \mathbf{H}_{-1}^{-1} \mathbf{E}_{-1} \mathbf{H}_{0}^{-1} \mathbf{J}_{0} \\
& +\mathbf{j}_{0} \mathbf{h}_{1} \mathbf{h}_{0}^{-1} \mathbf{j}_{0} \otimes \mathbf{J}_{0} \mathbf{F}_{-1} \mathbf{H}_{1} \mathbf{E}_{-1} \mathbf{H}_{0}^{-1} \mathbf{J}_{0} .
\end{aligned}
$$


By using $\mathbf{J}_{0} \mathbf{H}_{0} \mathbf{J}_{0}=1, \mathbf{J}_{0} \mathbf{K}_{0} \mathbf{J}_{0}=\hat{\mathbf{H}}_{1}$, and $\mathbf{J}_{0} \mathbf{F}_{0} \mathbf{E}_{0} \mathbf{J}_{0}=0$, we have

$$
\begin{aligned}
\mathbf{J}_{0} \mathbf{E}_{0} \mathbf{H}_{-1}^{-1} \mathbf{F}_{0} \mathbf{H}_{0} \mathbf{J}_{0} & =\mathbf{J}_{0} \mathbf{E}_{0} \mathbf{H}_{-1}^{-1} \mathbf{F}_{0} \mathbf{J}_{0}=\mathbf{J}_{0} \mathbf{H}^{-1} \mathbf{E}_{0} \mathbf{F}_{0} \mathbf{J}_{0} \\
& =\hat{\mathbf{H}}_{0}^{-1} \mathbf{J}_{0} \mathbf{E}_{0} \mathbf{F}_{0} \mathbf{J}_{0}=\hat{\mathbf{H}}_{0}^{-1} \mathbf{J}_{0} \frac{\mathbf{K}_{0}-\mathbf{K}_{0}^{-1}}{v-v^{-1}} \mathbf{J}_{0}=\hat{\mathbf{H}}_{0}^{-1} \frac{\hat{\mathbf{H}}_{1}-\hat{\mathbf{H}}_{1}^{-1}}{v-v^{-1}} .
\end{aligned}
$$

We also have

$$
\begin{aligned}
\mathbf{J}_{0} \mathbf{F}_{-1} \mathbf{H}_{1} \mathbf{F}_{0} \mathbf{H}_{0} \mathbf{J}_{0} & =\mathbf{J}_{0} \mathbf{F}_{-1} \mathbf{H}_{1} \mathbf{F}_{0} \mathbf{J}_{0}=\hat{\mathbf{H}}_{1} \hat{\mathbf{F}}_{0}, \\
\mathbf{J}_{0} \mathbf{E}_{0} \mathbf{H}_{-1}^{-1} \mathbf{E}_{-1} \mathbf{H}_{0}^{-1} \mathbf{J}_{0} & =\hat{\mathbf{H}}_{0}^{-1} \hat{\mathbf{E}}_{0}, \\
\mathbf{J}_{0} \mathbf{F}_{-1} \mathbf{H}_{1} \mathbf{E}_{-1} \mathbf{H}_{0}^{-1} \mathbf{J}_{0} & =\mathbf{J}_{0} \mathbf{H}_{1} \mathbf{J}_{0} \mathbf{J}_{0} \mathbf{F}_{-1} \mathbf{E}_{-1} \mathbf{J}_{0}=\hat{\mathbf{H}}_{1} \frac{\hat{\mathbf{H}}_{0}-\hat{\mathbf{H}}_{0}^{-1}}{v-v^{-1}} .
\end{aligned}
$$

Observe that $\mathbf{j}_{0} \mathbf{h}_{1} \mathbf{h}_{0} \mathbf{j}_{0}=v^{2} \hat{\mathbf{k}}_{0}$. By the above analysis, we have

$$
\begin{aligned}
\widetilde{\Delta}^{\mathfrak{c}}\left(\mathbf{j}_{0} \mathbf{e}_{0} \mathbf{f}_{0} \mathbf{j}_{0}\right) & =\mathbf{j}_{0} \mathbf{e}_{0} \mathbf{f}_{0} \mathbf{j}_{0} \otimes \hat{\mathbf{K}}_{0}+\hat{\mathbf{k}}_{0}^{-1} \otimes \hat{\mathbf{H}}_{0}^{-1} \frac{\hat{\mathbf{H}}_{1}-\hat{\mathbf{H}}_{1}^{-1}}{v-v^{-1}} \\
& +v^{2} \hat{\mathbf{k}}_{0} \otimes \hat{\mathbf{H}}_{1} \hat{\mathbf{F}}_{0}+v^{-2} \hat{\mathbf{k}}_{0}^{-1} \otimes \hat{\mathbf{H}}_{0}^{-1} \hat{\mathbf{E}}_{0}+\hat{\mathbf{k}}_{0} \otimes \hat{\mathbf{H}}_{1} \frac{\hat{\mathbf{H}}_{0}-\hat{\mathbf{H}}_{0}^{-1}}{v-v^{-1}}
\end{aligned}
$$

By definition, we also have

$$
\widetilde{\Delta}^{\mathfrak{c}}\left(\mathbf{j}_{0} \frac{\mathbf{k}_{0}^{-1}-\mathbf{k}_{0}}{v-v^{-1}} \mathbf{j}_{0}\right)=-\hat{\mathbf{k}}_{0} \otimes \frac{\hat{\mathbf{H}}_{1} \hat{\mathbf{H}}_{0}}{v-v^{-1}}+\hat{\mathbf{k}}_{0}^{-1} \otimes \frac{\hat{\mathbf{H}}_{1}^{-1} \hat{\mathbf{H}}_{0}^{-1}}{v-v^{-1}} .
$$

By adding the last two equalities, we have established the formula for $\widetilde{\Delta}^{\mathfrak{c}}\left(\hat{\mathbf{t}}_{0}\right)$.

We define the transfer map $\phi_{d, d-\mathfrak{n}}^{\imath \jmath}: \mathbf{S}_{\mathfrak{n}, d}^{\imath \jmath} \longrightarrow \mathbf{S}_{\mathfrak{n}, d-\mathfrak{n}}^{\imath \jmath}$ to be the composition

$$
\phi_{d, d-\mathfrak{n}}^{\imath \jmath}: \mathbf{S}_{\mathfrak{n}, d}^{\imath \jmath} \stackrel{\widetilde{\Delta}^{\mathfrak{c}}}{\longrightarrow} \mathbf{S}_{\mathfrak{n}, d-\mathfrak{n}}^{\imath \jmath} \otimes \mathbf{S}_{\mathfrak{n}, \mathfrak{n}} \stackrel{1 \otimes \chi_{\mathfrak{n}}}{\longrightarrow} \mathbf{S}_{\mathfrak{n}, d-\mathfrak{n}}^{\imath \jmath} .
$$

Recall that the "signed" homomorphism $\chi_{\mathfrak{n}}: \mathbf{S}_{\mathfrak{n}, \mathfrak{n}} \rightarrow \mathbb{Q}(v)$ satisfies that $\chi_{\mathfrak{n}}\left(\hat{\mathbf{E}}_{i}\right)=0, \chi_{\mathfrak{n}}\left(\hat{\mathbf{F}}_{i}\right)=$ 0 and $\chi_{\mathfrak{n}}\left(\hat{\mathbf{H}}_{i}\right)=v$. It follows by Proposition 8.2.1 that, for all $i \in[1, r]$,

$$
\phi_{d, d-\mathfrak{n}}^{\imath \jmath}\left(\hat{\mathbf{e}}_{i}\right)=\hat{\mathbf{e}}_{i}^{\prime}, \quad \phi_{d, d-\mathfrak{n}}^{\imath \jmath}\left(\hat{\mathbf{f}}_{i}\right)=\hat{\mathbf{f}}_{i}^{\prime}, \quad \phi_{d, d-\mathfrak{n}}^{\imath \jmath}\left(\hat{\mathbf{k}}_{i}^{ \pm 1}\right)=\hat{\mathbf{k}}_{i}^{\prime \pm 1}, \quad \phi_{d, d-\mathfrak{n}}^{\imath \jmath}\left(\hat{\mathbf{t}}_{0}\right)=\hat{\mathbf{t}}_{0}^{\prime} .
$$

Hence we have constructed projective systems $\left\{\left(\mathbf{S}_{\mathfrak{n}, d}^{\imath \jmath}, \phi_{d, d-\mathfrak{n}}^{\imath \jmath}\right)\right\}_{d \geqslant 0}$ and $\left\{\left(\mathbf{U}_{\mathfrak{n}, d}^{\imath \jmath}, \phi_{d, d-\mathfrak{n}}^{\imath \jmath}\right)\right\}_{d \geqslant 0}$.

We now describe the restriction of $\Delta^{\mathfrak{c}}: \mathbf{S}_{n, d}^{\mathfrak{c}} \longrightarrow \mathbf{S}_{n, d^{\prime}}^{\mathfrak{c}} \otimes \mathbf{S}_{n, d^{\prime \prime}}$ defined in (5.3.6) to the subalgebra $\mathbf{S}_{\mathfrak{n}, d}^{\imath \jmath}$, which shall be denoted by $\Delta^{\imath \jmath}$.

Proposition 8.2.2. We have a homomorphism $\Delta^{\imath \jmath}: \mathbf{S}_{\mathfrak{n}, d}^{\imath \jmath} \longrightarrow \mathbf{S}_{\mathfrak{n}, d^{\prime}}^{\imath \jmath} \otimes \mathbf{S}_{\mathfrak{n}, d^{\prime \prime}}$ and by restriction $\Delta^{\imath \jmath}: \mathbf{U}_{\mathfrak{n}, d}^{\imath \jmath} \longrightarrow \mathbf{U}_{\mathfrak{n}, d^{\prime}}^{\imath \jmath} \otimes \mathbf{U}_{\mathfrak{n}, d^{\prime \prime}}$. More precisely, for all $i \in[1, r]$, we have

$$
\begin{aligned}
\Delta^{\imath \jmath}\left(\hat{\mathbf{e}}_{i}\right) & =\hat{\mathbf{e}}_{i}^{\prime} \otimes \hat{\mathbf{K}}_{i}^{\prime \prime}+1 \otimes \hat{\mathbf{E}}_{i}^{\prime \prime}+\hat{\mathbf{k}}_{i}^{\prime} \otimes \hat{\mathbf{F}}_{n-1-i}^{\prime \prime} \hat{\mathbf{K}}_{i}^{\prime \prime}, \\
\Delta^{\imath \jmath}\left(\hat{\mathbf{f}}_{i}\right) & =\hat{\mathbf{f}}_{i}^{\prime} \otimes \hat{\mathbf{K}}_{n-1-i}^{\prime \prime}+\hat{\mathbf{k}}_{i}^{\prime-1} \otimes \hat{\mathbf{K}}_{n-1-i}^{\prime \prime} \hat{\mathbf{F}}_{i}^{\prime \prime}+1 \otimes \hat{\mathbf{E}}_{n-1-i}^{\prime \prime}, \\
\Delta^{\imath \jmath}\left(\hat{\mathbf{k}}_{i}\right) & =\hat{\mathbf{k}}_{i}^{\prime} \otimes \hat{\mathbf{K}}_{i}^{\prime \prime} \hat{\mathbf{K}}_{n-1-i}^{\prime \prime-1}, \\
\Delta^{\imath \jmath}\left(\hat{\mathbf{t}}_{0}\right) & =\hat{\mathbf{t}}_{0}^{\prime} \otimes \hat{\mathbf{K}}_{0}^{\prime \prime}+1 \otimes v \hat{\mathbf{K}}_{0}^{\prime \prime} \hat{\mathbf{F}}_{0}^{\prime \prime}+1 \otimes \hat{\mathbf{E}}_{0}^{\prime \prime}
\end{aligned}
$$

Proof. The first three formulas follow by $\Delta^{\imath \jmath}\left(\mathbf{j}_{0}\right)=\mathbf{j}_{0}^{\prime} \otimes \mathbf{J}_{0}^{\prime \prime}$ and Proposition 5.3.4. The last one can be obtained as that of Proposition 8.2.1, and we skip the detail. 
Since $\hat{\mathbf{e}}_{i}=\hat{\mathbf{f}}_{i}=0, \hat{\mathbf{t}}_{0}=1, \hat{\mathbf{k}}_{i}=v^{\delta_{i, r}} \in \mathbf{S}_{\mathfrak{n}, 0}^{\imath \jmath}$ for all $i \in[1, r]$, we have the following degenerate version of Proposition 8.2.2.

Proposition 8.2.3. We have an imbedding of algebras

$$
\vartheta_{\mathfrak{n}, d}=\left.\Delta^{\imath \jmath}\right|_{d^{\prime}=0}: \mathbf{S}_{\mathfrak{n}, d}^{\imath \jmath} \longrightarrow \mathbf{S}_{\mathfrak{n}, d}
$$

such that, for all $i \in[1, r]$,

$$
\begin{gathered}
\vartheta_{\mathfrak{n}, d}\left(\hat{\mathbf{e}}_{i}\right)=\hat{\mathbf{E}}_{i}+v^{\delta_{i, r}} \hat{\mathbf{F}}_{n-1-i} \hat{\mathbf{K}}_{i}, \quad \vartheta_{\mathfrak{n}, d}\left(\hat{\mathbf{f}}_{i}\right)=\hat{\mathbf{E}}_{n-1-i}+v^{-\delta_{i, r}} \hat{\mathbf{K}}_{n-1-i} \hat{\mathbf{F}}_{i}, \\
\vartheta_{\mathfrak{n}, d}\left(\hat{\mathbf{k}}_{i}\right)=v^{\delta_{i, r}} \hat{\mathbf{K}}_{i} \hat{\mathbf{K}}_{n-1-i}^{-1}, \quad \vartheta_{\mathfrak{n}, d}\left(\hat{\mathbf{t}}_{0}\right)=\hat{\mathbf{E}}_{0}+v \hat{\mathbf{K}}_{0} \hat{\mathbf{F}}_{0}+\hat{\mathbf{K}}_{0} .
\end{gathered}
$$

In particular, we have by restriction an imbedding of algebras $\vartheta_{\mathfrak{n}, d}: \mathbf{U}_{\mathfrak{n}, d}^{\imath \jmath} \longrightarrow \mathbf{U}_{\mathfrak{n}, d}$.

Following Definition 7.3.1, a notation of a $\vartheta$-aperiodic matrix in $\Xi_{\mathfrak{n}, d}^{\imath \jmath}$ is self-explanatory. The following is a counterpart of Proposition 7.3.4, whose proof will be skipped.

Proposition 8.2.4. The algebra $\mathbf{U}_{\mathfrak{n}, d}^{\imath \jmath}$ has a canonical basis $\left\{\{A\}_{d} \mid A \in \Xi_{\mathfrak{n}, d}^{\imath \jmath}\right.$ ๖-aperiodic $\}$.

\subsection{QUANTUM SYMMETRIC PAIR $\left(\mathbf{U}\left(\widehat{\mathfrak{s l}}_{\mathfrak{n}}\right), \mathbf{U}_{\mathfrak{n}}^{\imath \jmath}\right)$ AND CANONICAL BASIS ON $\dot{\mathbf{U}}_{\mathfrak{n}}^{\imath \jmath}$}

The results in Chapter 7, in particular those in Sections 7.3 7.4, admit $\urcorner$-counterparts with basically identical proofs; we shall outline these below.

Starting with the projective system $\left\{\left(\mathbf{U}_{\mathfrak{n}, d}^{\imath \jmath}, \phi_{d, d-\mathfrak{n}}^{\imath \jmath}\right)\right\}_{d \in \mathbb{N}}$, we construct two distinguished algebras $\mathbf{U}_{\mathfrak{n}}^{\imath \jmath}$ and $\dot{\mathbf{U}}_{\mathfrak{n}}^{\imath \jmath}$ out of its associated projective limit algebra $\mathbf{U}_{\mathfrak{n}, \infty}^{\imath \jmath}$; the Chevalley generators of $\mathbf{U}_{\mathfrak{n}}^{\imath \jmath}$ are denoted again by $\hat{\mathbf{e}}_{i}, \hat{\mathbf{f}}_{i}, \hat{\mathbf{k}}_{i}^{ \pm 1}$, for $i \in[1, r]$, and $\hat{\mathbf{t}}_{0}$. The family of imbeddings $\left\{\vartheta_{\mathfrak{n}, d}: \mathbf{U}_{\mathfrak{n}, d}^{\imath \jmath} \rightarrow \mathbf{U}_{\mathfrak{n}, d}\right\}_{d \geqslant 1}$ induces an algebra imbedding $\vartheta_{\mathfrak{n}}: \mathbf{U}_{\mathfrak{n}}^{\imath \jmath} \rightarrow \mathbf{U}_{\mathfrak{n}}$. The family of $\Delta^{\imath \jmath}$ (for various $d^{\prime}, d^{\prime \prime}$ ) induces an algebra homomorphism $\Delta^{\imath \jmath}: \mathbf{U}_{\mathfrak{n}}^{\imath \jmath} \rightarrow \mathbf{U}_{\mathfrak{n}}^{\imath \jmath} \otimes \mathbf{U}_{\mathfrak{n}}$, whose action on the Chevalley generators can be presented explicitly. Recall the algebra isomorphism $\mathbf{U}_{\mathfrak{n}} \cong \mathbf{U}\left(\widehat{\mathfrak{s l}}_{\mathfrak{n}}\right)$. Summarizing we have established the following.

Theorem 8.3.1. The pair $\left(\mathbf{U}\left(\widehat{\mathfrak{s l}}_{\mathfrak{n}}\right), \mathbf{U}_{\mathfrak{n}}^{\imath \jmath}\right)$ forms a quantum symmetric pair of affine type. (see Figure 3 for the relevant involution.)

Recalling $\widetilde{\Xi}_{n}$ from (6.2.2), we introduce the following subsets of $\widetilde{\Xi}_{n}$ :

$$
\begin{aligned}
\widetilde{\Xi}_{\mathfrak{n}}^{\imath \jmath} & =\left\{A=\left(a_{i j}\right) \in \widetilde{\Xi}_{n} \mid a_{0, j}=\delta_{0, j}, a_{i, 0}=\delta_{i, 0}\right\}, \\
\widetilde{\Xi}_{\mathfrak{n}}^{\imath, a p} & =\left\{A \in \widetilde{\Xi}_{\mathfrak{n}}^{\imath \jmath} \mid A \text { is } \imath \text {-aperiodic }\right\} .
\end{aligned}
$$

We define an equivalence relation $\approx$ on $\widetilde{\Xi}_{\mathfrak{n}}^{\imath \jmath}, a p$ as in (6.2.4) and let $\hat{A}$ be the equivalence class of $A$. A hybrid monomial basis $\left\{h_{A}\right\}$ for $\mathbf{S}_{\mathfrak{n}, d}^{\imath \jmath}$ can be constructed (similar to Proposition 7.3.5 in $\jmath$ type), and it gives rise to a monomial basis $\left\{h_{\hat{A}} \mid \widehat{A} \in \widetilde{\Xi}_{\mathfrak{n}}^{\imath, a p} / \approx\right\}$ for the algebra $\dot{\mathbf{U}}_{\mathfrak{n}}^{\imath \jmath}$. A bilinear form $\langle\cdot, \cdot\rangle$ on $\dot{\mathbf{U}}_{\mathfrak{n}}^{\imath \jmath}$ can be defined similarly as in Section 6.3 and shown to be nondegenerate. We have the following analogue of Theorem 7.4 .3 (and also of Theorems 6.4.3 and 6.4.5).

Theorem 8.3.2. There exists a canonical basis $\dot{\mathbf{B}}_{\mathfrak{n}}^{\imath \jmath}=\left\{b_{\widehat{A}} \mid \widehat{A} \in \widetilde{\Xi}_{\mathfrak{n}}^{\imath \jmath}\right.$,ap $\left./ \approx\right\}$ for $\dot{\mathbf{U}}_{\mathfrak{n}}^{\imath \jmath}$, whose transition matrix with respect to the monomial basis is uni-triangular. Moreover, the structure constants of the canonical basis $\dot{\mathbf{B}}_{\mathfrak{n}}^{\text {ju }}$ are positive integral, i.e., they all lie in $\mathbb{N}\left[v, v^{-1}\right]$ with respect to the multiplication and comultiplication, and lie in $v^{-1} \mathbb{N}\left[\left[v^{-1}\right]\right]$ with respect to the bilinear pairing . 


\subsection{The SChur Algebras OF TYPE $\imath \imath$}

Recall $\eta=n-2$, and so $\eta=\mathfrak{n}-1=2 r$ for $r \geqslant 1$. We set

$$
\Xi_{\eta, d}^{\imath \imath}=\Xi_{\mathfrak{n}, d}^{\jmath \imath} \cap \Xi_{\mathfrak{n}, d}^{\imath \jmath}, \quad \mathbf{j}_{r, 0}=\mathbf{j}_{r} \mathbf{j}_{0} .
$$

The idempotent $\mathbf{j}_{r, 0}$ gives rise to the subalgebra $\mathbf{S}_{\eta, d}^{n}$ :

$$
\mathbf{S}_{\eta, d}^{n}=\mathbf{j}_{r, 0} \mathbf{S}_{n, d}^{\mathfrak{c}} \mathbf{j}_{r, 0}=\mathbf{S}_{\mathfrak{n}, d}^{\jmath \imath} \cap \mathbf{S}_{\mathfrak{n}, d}^{\imath \jmath}
$$

Let $\mathbf{U}_{\eta, d}^{\imath}$ be the subalgebra of $\mathbf{S}_{\eta, d}^{\imath}$ generated by the following Chevalley generators:

$$
\begin{aligned}
\tilde{\mathbf{e}}_{i} & =\mathbf{j}_{r, 0} \check{\mathbf{e}}_{i} \mathbf{j}_{r, 0}, \quad \tilde{\mathbf{f}}_{i}=\mathbf{j}_{r, 0} \check{\mathbf{f}}_{i} \mathbf{j}_{r, 0}, \\
\tilde{\mathbf{k}}_{i}^{ \pm 1} & =\check{\mathbf{j}}_{r, 0} \check{\mathbf{k}}_{i}^{ \pm 1} \mathbf{j}_{r, 0}, \quad \forall i \in[1, r-1], \\
\tilde{\mathbf{h}}_{a}^{ \pm 1} & =\mathbf{j}_{r, 0} \check{\mathbf{h}}_{a}^{ \pm 1} \mathbf{j}_{r, 0}, \quad \forall a \in[0, r], \\
\tilde{\mathbf{t}}_{0} & =\mathbf{j}_{r, 0}\left(\check{\mathbf{e}}_{0} \check{\mathbf{f}}_{0}+\frac{\check{\mathbf{k}}_{0}^{-1}-\check{\mathbf{k}}_{0}}{v-v^{-1}}\right) \mathbf{j}_{r, 0}=\mathbf{j}_{r, 0} \hat{\mathbf{t}}_{0} \mathbf{j}_{r, 0}, \\
\tilde{\mathbf{t}}_{r} & =\mathbf{j}_{r, 0} \check{\mathbf{t}}_{r} \mathbf{j}_{r, 0} .
\end{aligned}
$$

Note that $\tilde{\mathbf{e}}_{i}=\mathbf{j}_{r, 0} \mathbf{e}_{i} \mathbf{j}_{r, 0}=\mathbf{j}_{r, 0} \hat{\mathbf{e}}_{i} \mathbf{j}_{r, 0}$, etc.

We shall also need a type $A$ counterpart of the above construction as follows. We set

$$
\Theta_{\eta, d}^{\imath}=\Theta_{\mathfrak{n}, d}^{\jmath \imath} \cap \Theta_{\mathfrak{n}, d}^{\imath \jmath}, \quad \mathbf{J}_{r, 0}=\mathbf{J}_{r} \mathbf{J}_{0}, \quad \mathbf{S}_{\eta, d}=\mathbf{J}_{r, 0} \mathbf{S}_{n, d} \mathbf{J}_{r, 0} .
$$

Let $\mathbf{U}_{\eta, d}$ be the subalgebra of $\mathbf{S}_{\eta, d}$ generated by the following Chevalley generators:

$$
\begin{aligned}
\tilde{\mathbf{E}}_{i} & = \begin{cases}\mathbf{J}_{r, 0} \check{\mathbf{E}}_{0} \check{\mathbf{E}}_{-1} \mathbf{J}_{r, 0}, & \text { if } i=0, \\
\mathbf{J}_{r, 0} \check{\mathbf{E}}_{i} \mathbf{J}_{r, 0}, & \text { if } i \in[1, \eta-1] .\end{cases} \\
\tilde{\mathbf{F}}_{i} & = \begin{cases}\mathbf{J}_{r, 0} \check{\mathbf{F}}_{-1} \check{\mathbf{F}}_{0} \mathbf{J}_{r, 0}, & \text { if } i=0, \\
\mathbf{J}_{r, 0} \check{\mathbf{F}}_{i} \mathbf{J}_{r, 0}, & \text { if } i \in[1, \eta-1] .\end{cases} \\
\tilde{\mathbf{K}}_{i}^{ \pm 1} & = \begin{cases}\mathbf{J}_{r, 0} \check{\mathbf{K}}_{0}^{ \pm 1} \check{\mathbf{K}}_{-1}^{ \pm 1} \mathbf{J}_{r, 0}, & \text { if } i=0, \\
\mathbf{J}_{r, 0} \check{\mathbf{K}}_{i}^{ \pm 1} \mathbf{J}_{r, 0}, & \text { if } i \in[1, \eta-1] .\end{cases} \\
\tilde{\mathbf{H}}_{a}^{ \pm 1} & = \begin{cases}\mathbf{J}_{r, 0} \check{\mathbf{H}}_{-1}^{ \pm 1} \mathbf{J}_{r, 0}, & \text { if } a=0, \\
\mathbf{J}_{r, 0} \check{\mathbf{H}}_{a}^{ \pm 1} \mathbf{J}_{r, 0}, & \text { if } a \in[1, \eta] .\end{cases}
\end{aligned}
$$

We can make the indices periodic by setting $\tilde{\mathbf{E}}_{i}=\tilde{\mathbf{E}}_{i+\eta}$, etc, i.e., $i \in \mathbb{Z} / \eta \mathbb{Z}$.

Let us describe the restriction to the subalgebra $\mathbf{U}_{\eta, d}^{n}$ (denoted by the same notation) of $\widetilde{\Delta}^{\mathfrak{c}}: \mathbf{S}_{n, d}^{\mathfrak{c}} \rightarrow \mathbf{S}_{n, d^{\prime}}^{\mathfrak{c}} \otimes \mathbf{S}_{n, d^{\prime \prime}}$ from (5.2.1), for arbitrary $d^{\prime}, d^{\prime \prime}$ such that $d=d^{\prime}+d^{\prime \prime}$. The proof is similar to that for Proposition 8.2.1 and will be skipped. 
Proposition 8.4.1. We have an algebra homomorphism $\widetilde{\Delta}^{\mathfrak{c}}: \mathbf{U}_{\eta, d}^{\imath \imath} \rightarrow \mathbf{U}_{\eta, d^{\prime}}^{\imath \imath} \otimes \mathbf{U}_{\eta, d^{\prime \prime}}$. More precisely, for all $i \in[1, r-1]$, we have

$$
\begin{aligned}
\widetilde{\Delta}^{\mathfrak{c}}\left(\tilde{\mathbf{e}}_{i}\right) & =\tilde{\mathbf{e}}_{i}^{\prime} \otimes \tilde{\mathbf{H}}_{i+1}^{\prime \prime} \tilde{\mathbf{H}}_{\mathfrak{n}-1-i}^{\prime \prime-1}+\tilde{\mathbf{h}}_{i+1}^{\prime-1} \otimes \tilde{\mathbf{E}}_{i}^{\prime \prime} \hat{\mathbf{H}}_{\mathfrak{n}-1-i}^{\prime \prime-1}+\tilde{\mathbf{h}}_{i+1}^{\prime} \otimes \tilde{\mathbf{F}}_{\mathfrak{n}-1-i}^{\prime \prime} \tilde{\mathbf{H}}_{i+1}^{\prime \prime}, \\
\widetilde{\Delta}^{\mathfrak{c}}\left(\tilde{\mathbf{f}}_{i}\right) & =\tilde{\mathbf{f}}_{i}^{\prime} \otimes \tilde{\mathbf{H}}_{i}^{\prime \prime-1} \tilde{\mathbf{H}}_{\mathfrak{n}-i}^{\prime \prime}+\tilde{\mathbf{h}}_{i}^{\prime} \otimes \tilde{\mathbf{F}}_{i}^{\prime \prime} \tilde{\mathbf{H}}_{\mathfrak{n}-i}^{\prime \prime}+\tilde{\mathbf{h}}_{i}^{\prime-1} \otimes \tilde{\mathbf{E}}_{\mathfrak{n}-1-i}^{\prime \prime} \tilde{\mathbf{H}}_{i}^{\prime \prime-1}, \\
\tilde{\Delta}^{\mathfrak{c}}\left(\tilde{\mathbf{k}}_{i}\right) & =\tilde{\mathbf{k}}_{i}^{\prime} \otimes \tilde{\mathbf{K}}_{i}^{\prime \prime} \tilde{\mathbf{K}}_{\mathfrak{n}-1-i}^{\prime \prime-1} \\
\widetilde{\Delta}^{\mathfrak{c}}\left(\tilde{\mathbf{t}}_{0}\right) & =\tilde{\mathbf{t}}_{0}^{\prime} \otimes \tilde{\mathbf{K}}_{0}^{\prime \prime}+v^{2} \tilde{\mathbf{k}}_{0}^{\prime} \otimes \tilde{\mathbf{H}}_{1}^{\prime \prime} \tilde{\mathbf{F}}_{0}^{\prime \prime}+v^{-2} \tilde{\mathbf{k}}_{0}^{\prime-1} \otimes \tilde{\mathbf{H}}_{0}^{\prime \prime-1} \tilde{\mathbf{E}}_{0}^{\prime \prime} \\
\widetilde{\Delta}^{\mathfrak{c}}\left(\tilde{\mathbf{t}}_{r}\right) & =\tilde{\mathbf{t}}_{r}^{\prime} \otimes \tilde{\mathbf{K}}_{r}^{\prime \prime}+v^{2} \tilde{\mathbf{k}}_{r}^{\prime-1} \otimes \tilde{\mathbf{H}}_{r+1}^{\prime \prime} \tilde{\mathbf{F}}_{r}^{\prime \prime}+v^{-2} \tilde{\mathbf{k}}_{r}^{\prime} \otimes \tilde{\mathbf{H}}_{r}^{\prime \prime-1} \tilde{\mathbf{E}}_{r}^{\prime \prime}
\end{aligned}
$$

We define the transfer map $\phi_{d, d-\eta}^{\imath \imath}: \mathbf{S}_{\eta, d}^{\imath \imath} \longrightarrow \mathbf{S}_{\eta, d-\eta}^{\imath \imath}$ to be the composition of the following homomorphisms

$$
\phi_{d, d-\eta}^{\imath \imath}: \mathbf{S}_{\eta, d}^{\imath \imath} \stackrel{\widetilde{\Delta}^{c}}{\longrightarrow} \mathbf{S}_{\eta, d-\eta}^{\imath \imath} \otimes \mathbf{S}_{\eta, \eta} \stackrel{1 \otimes \chi_{\eta}}{\longrightarrow} \mathbf{S}_{\eta, d-\eta}^{\imath \imath} .
$$

Noting that $\chi_{\eta}\left(\tilde{\mathbf{E}}_{i}\right)=0, \chi_{\eta}\left(\tilde{\mathbf{F}}_{i}\right)=0$ and $\chi_{\eta}\left(\tilde{\mathbf{H}}_{i}\right)=v$, we have, for all $i \in[1, r-1]$,

$$
\begin{array}{ll}
\phi_{d, d-\eta}^{\imath \imath}\left(\tilde{\mathbf{e}}_{i}\right)=\tilde{\mathbf{e}}_{i}^{\prime}, & \phi_{d, d-\eta}^{\imath \imath}\left(\tilde{\mathbf{f}}_{i}\right)=\tilde{\mathbf{f}}_{i}^{\prime}, \quad \phi_{d, d-\eta}^{\imath \imath}\left(\tilde{\mathbf{k}}_{i}^{ \pm 1}\right)=\tilde{\mathbf{k}}_{i}^{\prime \pm 1}, \\
\phi_{d, d-\eta}^{\imath \imath}\left(\tilde{\mathbf{t}}_{0}\right)=\tilde{\mathbf{t}}_{0}^{\prime}, & \phi_{d, d-\eta}^{\imath \imath}\left(\tilde{\mathbf{t}}_{r}\right)=\tilde{\mathbf{t}}_{r}^{\prime} .
\end{array}
$$

We now describe the restriction of $\Delta^{\mathfrak{c}}(5.3 .6)$ to the subalgebra $\mathbf{S}_{\eta, d}^{\imath \imath}$, which shall be denoted by $\Delta^{\imath}$. We shall skip the proof, as it is similar to earlier cases.

Proposition 8.4.2. We have a homomorphism $\Delta^{\imath \imath}: \mathbf{S}_{\eta, d}^{\imath \imath} \rightarrow \mathbf{S}_{\eta, d^{\prime}}^{\imath \imath} \otimes \mathbf{S}_{\eta, d^{\prime \prime}}$, and by restriction, a homomorphism $\Delta^{\imath \imath}: \mathbf{U}_{\eta, d}^{\imath \imath} \rightarrow \mathbf{U}_{\eta, d^{\prime}}^{\imath \imath} \otimes \mathbf{U}_{\eta, d^{\prime \prime}}$. More precisely, for all $i \in[1, r-1]$, we have

$$
\begin{aligned}
\Delta^{\imath}\left(\tilde{\mathbf{e}}_{i}\right) & =\tilde{\mathbf{e}}_{i}^{\prime} \otimes \tilde{\mathbf{K}}_{i}^{\prime \prime}+1 \otimes \tilde{\mathbf{E}}_{i}^{\prime \prime}+\tilde{\mathbf{k}}_{i}^{\prime} \otimes \tilde{\mathbf{F}}_{\mathfrak{n}-1-i}^{\prime \prime} \tilde{\mathbf{K}}_{i}^{\prime \prime}, \\
\Delta^{\imath \imath}\left(\tilde{\mathbf{f}}_{i}\right) & =\tilde{\mathbf{f}}_{i}^{\prime} \otimes \tilde{\mathbf{K}}_{\mathfrak{n}-1-i}^{\prime \prime}+\tilde{\mathbf{k}}_{i}^{\prime-1} \otimes \tilde{\mathbf{K}}_{\mathfrak{n}-1-i}^{\prime \prime} \tilde{\mathbf{F}}_{i}^{\prime \prime}+1 \otimes \tilde{\mathbf{E}}_{\mathfrak{n}-1-i}^{\prime \prime}, \\
\Delta^{\imath}\left(\tilde{\mathbf{k}}_{i}\right) & =\tilde{\mathbf{k}}_{i}^{\prime} \otimes \tilde{\mathbf{K}}_{i}^{\prime \prime} \tilde{\mathbf{K}}_{\mathfrak{n}-1-i}^{\prime \prime}, \\
\Delta^{\imath}\left(\tilde{\mathbf{t}}_{0}\right) & =\tilde{\mathbf{t}}_{0}^{\prime} \otimes \tilde{\mathbf{K}}_{0}^{\prime \prime}+1 \otimes v \tilde{\mathbf{K}}_{0}^{\prime \prime} \tilde{\mathbf{F}}_{0}^{\prime \prime}+1 \otimes \tilde{\mathbf{E}}_{0}^{\prime \prime}, \\
\Delta^{\imath}\left(\tilde{\mathbf{t}}_{r}\right) & =\tilde{\mathbf{t}}_{r}^{\prime} \otimes \tilde{\mathbf{K}}_{r}^{\prime \prime}+1 \otimes v \tilde{\mathbf{K}}_{r}^{\prime \prime} \tilde{\mathbf{F}}_{r}^{\prime \prime}+1 \otimes \tilde{\mathbf{E}}_{r}^{\prime \prime} .
\end{aligned}
$$

A degenerate version of Proposition 8.4 .2 gives us the following description for the homomorphism $u_{\eta, d}=\left.\Delta^{\imath \imath}\right|_{d^{\prime}=0}: \mathbf{S}_{\eta, d}^{\imath \imath} \longrightarrow \mathbf{S}_{\eta, d}$.

Proposition 8.4.3. We have imbeddings of algebras

$$
u_{\eta, d}: \mathbf{S}_{\eta, d}^{\imath \imath} \longrightarrow \mathbf{S}_{\eta, d}, \quad u_{\eta, d}: \mathbf{U}_{\eta, d}^{\imath \imath} \longrightarrow \mathbf{U}_{\eta, d}
$$

Moreover, for all $i \in[1, r-1]$, we have

$$
\begin{aligned}
u_{\eta, d}\left(\tilde{\mathbf{e}}_{i}\right) & =\tilde{\mathbf{E}}_{i}+\tilde{\mathbf{F}}_{\mathfrak{n}-1-i} \tilde{\mathbf{K}}_{i}=\tilde{\mathbf{E}}_{i}+\tilde{\mathbf{F}}_{-i} \tilde{\mathbf{K}}_{i}, \\
u_{\eta, d}\left(\tilde{\mathbf{f}}_{i}\right) & =\tilde{\mathbf{E}}_{\mathfrak{n}-1-i}+\tilde{\mathbf{K}}_{\mathfrak{n}-1-i} \tilde{\mathbf{F}}_{i}=\tilde{\mathbf{E}}_{-i}+\tilde{\mathbf{K}}_{-i} \tilde{\mathbf{F}}_{i}, \\
u_{\eta, d}\left(\tilde{\mathbf{k}}_{i}\right) & =\tilde{\mathbf{K}}_{i} \tilde{\mathbf{K}}_{\mathfrak{n}-1-i}^{-1}=\tilde{\mathbf{K}}_{i} \tilde{\mathbf{K}}_{-i}^{-1} \\
u_{\eta, d}\left(\tilde{\mathbf{t}}_{0}\right) & =\tilde{\mathbf{E}}_{0}+v \tilde{\mathbf{K}}_{0} \tilde{\mathbf{F}}_{0}+\tilde{\mathbf{K}}_{0} \\
u_{\eta, d}\left(\tilde{\mathbf{t}}_{r}\right) & =\tilde{\mathbf{E}}_{r}+v \tilde{\mathbf{K}}_{r} \tilde{\mathbf{F}}_{r}+\tilde{\mathbf{K}}_{r}
\end{aligned}
$$




\subsection{Realization of A NEW COIDEAL SUBAlgEBRA $\mathbf{U}_{\eta}^{\imath \imath}$}

We first formulate quickly results on monomial and canonical bases for $\mathbf{U}_{\eta, d}^{\imath \imath}$ analogous to Lusztig algebras of types $\jmath \jmath, \jmath, \imath$ treated earlier. Recall $\Xi_{\eta, d}^{\imath \imath}$ from (8.4.1). Following Definition 7.3.1, a notation of a $u$-aperiodic matrix $A$ in $\Xi_{\eta, d}^{\imath \imath}$ is self-explanatory. Similar to Proposition 7.3.4 (also see Proposition 8.2.4) we can establish the canonical basis for $\mathbf{U}_{\eta, d}^{\imath \imath}$. This is again based on the existence of a monomial basis $\left\{y_{A}\right\}$ for $\mathbf{U}_{\eta, d}^{\imath \imath}$, which can be established in a way similar to Proposition 7.3.3. A hybrid monomial basis $\left\{h_{A}\right\}$ for $\mathbf{U}_{\eta, d}^{\imath \imath}$ can also be established in a way similar to Proposition 7.3.5. We summarize these as follows.

Proposition 8.5.1. The algebra $\mathbf{U}_{\eta, d}^{\imath \imath}$ admits a monomial basis $\left\{y_{A} \mid A \in \Xi_{\eta, d}^{\imath \imath}\right.$ n-aperiodic $\}$ as well as a hybrid monomial basis $\left\{h_{A} \mid A \in \Xi_{\eta, d}^{n \imath}\right.$ u-aperiodic $\}$. Also, the set $\left\{\{A\}_{d} \mid A \in\right.$ $\Xi_{\eta, d}^{\imath \imath}$ u-aperiodic $\}$ forms a canonical basis for $\mathbf{U}_{\eta, d}^{\imath \imath}$.

Example 8.5.2. Let $r=1$, hence $\eta=2$. Consider the following matrix $A$ in $\Xi_{d}^{\imath 2}$ after deleting zero and second row and columns.

\begin{tabular}{c|c|c|c|c|c|c|c|c|c|c} 
& $\mathrm{c}-3$ & $\mathrm{c}-2$ & $\mathrm{c}-1$ & $\mathrm{c} 0$ & $\mathrm{c} 1$ & $\mathrm{c} 2$ & $\mathrm{c} 3$ & $\mathrm{c} 4$ & $\mathrm{c} 5$ & $\mathrm{c} 6$ \\
\hline & & & & & & & & & & \\
\hline $\mathrm{r} 0$ & 0 & 0 & 2 & $*$ & 0 & 3 & 4 & & & \\
\hline $\mathrm{r} 1$ & & 4 & 3 & 0 & $*$ & 2 & 0 & 0 & & \\
\hline $\mathrm{r} 2$ & & & 0 & 0 & 2 & $*$ & 0 & 3 & 4 & \\
\hline $\mathrm{r} 3$ & & & & 4 & 3 & 0 & $*$ & 2 & 0 & 0 \\
\hline & & & & & & & & & &
\end{tabular}

Then we have

$$
\tilde{\mathbf{t}}_{0}^{\langle 7\rangle} * \tilde{\mathbf{t}}_{1}^{\langle 9\rangle} * \tilde{\mathbf{t}}_{0}^{\langle 4\rangle} * 1_{\operatorname{co}(A)}=[A]+\text { lower terms }
$$

where

$$
\tilde{\mathbf{t}}_{0}^{\langle R\rangle}=\sum_{X: X-R E_{\theta, \eta}^{0,1} \text { diagonal }}[X], \quad \tilde{\mathbf{t}}_{1}^{\langle R\rangle}=\sum_{X: X-R E_{\theta, \eta}^{2,1} \text { diagonal }}[X] .
$$

This is a typical monomial appearing in a monomial basis of $\mathbf{S}_{2, d}^{\imath 2}$.

Now we shall formulate the $\imath$-counterparts of the results on coideal algebras arising from families of Lusztig algebras in Sections 7.4 and 8.3. Again we skip the proofs as they are analogous to the earlier cases.

Starting with the projective system $\left\{\left(\mathbf{U}_{\eta, d}^{\imath \imath}, \phi_{d, d-\eta}^{\imath \imath}\right)\right\}_{d \in \mathbb{N}}$, we construct two distinguished algebras $\mathbf{U}_{\eta}^{\imath \imath}$ and $\dot{\mathbf{U}}_{\eta}^{\imath \imath}$ out of its associated limit algebra $\mathbf{U}_{\eta, \infty}^{\imath}$; the Chevalley generators of $\mathbf{U}_{\eta}^{\imath \imath}$ are denoted again by $\tilde{\mathbf{t}}_{0}, \tilde{\mathbf{t}}_{r}, \tilde{\mathbf{e}}_{i}, \tilde{\mathbf{f}}_{i}, \tilde{\mathbf{k}}_{i}^{ \pm 1}$, for $i \in[1, r-1]$. The family of imbeddings $\left\{\imath_{\eta, d}: \mathbf{U}_{\eta, d}^{\imath \imath} \rightarrow \mathbf{U}_{\eta, d}\right\}_{d \geqslant 1}$ induces an algebra imbedding $\imath_{\eta}: \mathbf{U}_{\eta}^{\imath \imath} \rightarrow \mathbf{U}_{\eta}$. The family of $\Delta^{\imath}$ (for various $d^{\prime}, d^{\prime \prime}$ ) induces an algebra homomorphism $\Delta^{\imath \imath}: \mathbf{U}_{\eta}^{\imath \imath} \rightarrow \mathbf{U}_{\eta}^{\imath \imath} \otimes \mathbf{U}_{\eta}$, whose action on the Chevalley generators can be presented explicitly. Recall the algebra isomorphism $\mathbf{U}_{\eta} \cong \mathbf{U}\left(\hat{\mathfrak{s l}}_{\eta}\right)$. Summarizing we have established the following.

Theorem 8.5.3. The pair $\left(\mathbf{U}\left(\widehat{\mathfrak{s l}}_{\eta}\right), \mathbf{U}_{\eta}^{\imath \imath}\right)$ forms a quantum symmetric pair of affine type. (see Figure 4 for the relevant involution.) 
Recalling $\widetilde{\Xi}_{\mathfrak{n}}^{\jmath}$ from (7.4.1) and $\widetilde{\Xi}_{\mathfrak{n}}^{\imath \jmath}$ from (8.3.1), we introduce the following subsets of $\widetilde{\Xi}_{n}$ :

$$
\widetilde{\Xi}_{\eta}^{\imath \imath}=\widetilde{\Xi}_{\mathfrak{n}}^{\jmath} \cap \widetilde{\Xi}_{\mathfrak{n}}^{\imath \jmath}, \quad \widetilde{\Xi}_{\eta}^{\imath, a p}=\left\{A \in \widetilde{\Xi}_{\eta}^{\imath \imath} \mid A \text { is } \imath \text {-aperiodic }\right\} .
$$

We have the following $\imath$-analogue of Theorem 7.4 .3 and Theorems 8.3 .2 ,

Theorem 8.5.4. There exists a canonical basis $\dot{\mathbf{B}}_{\eta}^{\imath \imath}=\left\{b_{\widehat{A}} \mid \widehat{A} \in \widetilde{\Xi}_{\eta}^{\imath \imath, a p} / \approx\right\}$ for $\dot{\mathbf{U}}_{\eta}^{\imath \imath}$, whose transition matrix with respect to the monomial basis is uni-triangular. Moreover, the structure constants of the canonical basis $\dot{\mathbf{B}}_{\eta}^{\imath}$ all lie in $\mathbb{N}\left[v, v^{-1}\right]$ with respect to the multiplication and comultiplication, and in $v^{-1} \mathbb{N}\left[\left[v^{-1}\right]\right]$ with respect to the bilinear pairing .

Recall the Cartan integers $c_{i j}$ from (5.1.7). We now give a presentation for the algebra $\mathbf{U}_{\eta}^{\imath}$, which is a counterpart of Propositions 6.1.5 and 7.4.2. This presentation is again a variant of [Ko14, Theorem 7.1] in our setting and our notation.

Proposition 8.5.5. Let $r \geqslant 2$ and so $\eta=2 r \geqslant 4$. The $\mathbb{Q}(v)$-algebra $\mathbf{U}_{\eta}^{\imath 2}$ has a presentation with generators $\tilde{\mathbf{e}}_{i}, \tilde{\mathbf{f}}_{i}, \tilde{\mathbf{k}}_{i}^{ \pm 1}$ for $i \in[1, r-1]$ and $\check{\mathbf{t}}_{k}$ for $k=0, r$ and the following relations for all $i, j \in[1, r-1], k \in\{0, r\}$ :

$$
\begin{aligned}
& \tilde{\mathbf{k}}_{1}^{2} \cdots \tilde{\mathbf{k}}_{r-1}^{2}=1, \\
& \tilde{\mathbf{k}}_{i} \tilde{\mathbf{k}}_{i}^{-1}=1, \quad \tilde{\mathbf{k}}_{i} \tilde{\mathbf{k}}_{j}=\tilde{\mathbf{k}}_{j} \tilde{\mathbf{k}}_{i}, \\
& \tilde{\mathbf{k}}_{i} \tilde{\mathbf{e}}_{j} \tilde{\mathbf{k}}_{i}^{-1}=v^{c_{i j}} \tilde{\mathbf{e}}_{j}, \quad \tilde{\mathbf{k}}_{i} \tilde{\mathbf{f}}_{j} \tilde{\mathbf{k}}_{i}^{-1}=v^{-c_{i j}} \tilde{\mathbf{f}}_{j}, \\
& \tilde{\mathbf{k}}_{i} \tilde{\mathbf{t}}_{k}=\tilde{\mathbf{t}}_{k} \tilde{\mathbf{k}}_{i}, \quad \tilde{\mathbf{t}}_{0} \tilde{\mathbf{t}}_{r}=\tilde{\mathbf{t}}_{r} \tilde{\mathbf{t}}_{0}, \\
& \tilde{\mathbf{e}}_{i} \tilde{\mathbf{e}}_{j}=\tilde{\mathbf{e}}_{j} \tilde{\mathbf{e}}_{i}, \quad \tilde{\mathbf{f}}_{i} \tilde{\mathbf{f}}_{j}=\tilde{\mathbf{f}}_{j} \tilde{\mathbf{f}}_{i}, \quad \forall|i-j|>1, \\
& \tilde{\mathbf{e}}_{i} \tilde{\mathbf{t}}_{k}=\tilde{\mathbf{t}}_{k} \tilde{\mathbf{e}}_{i}, \quad \tilde{\mathbf{f}}_{i} \tilde{\mathbf{t}}_{k}=\tilde{\mathbf{t}}_{k} \tilde{\mathbf{f}}_{i}, \quad \forall|i-k|>1, \\
& \tilde{\mathbf{e}}_{i}^{2} \tilde{\mathbf{e}}_{j}+\tilde{\mathbf{e}}_{j} \tilde{\mathbf{e}}_{i}^{2}=\left(v+v^{-1}\right) \tilde{\mathbf{e}}_{i} \tilde{\mathbf{e}}_{j} \tilde{\mathbf{e}}_{i}, \quad \forall|i-j|=1, \\
& \tilde{\mathbf{f}}_{i}^{2} \tilde{\mathbf{f}}_{j}+\tilde{\mathbf{f}}_{j} \tilde{\mathbf{f}}_{i}^{2}=\left(v+v^{-1}\right) \tilde{\mathbf{f}}_{i} \tilde{\mathbf{f}}_{j} \tilde{\mathbf{f}}_{i}, \quad \forall|i-j|=1, \\
& \tilde{\mathbf{e}}_{i}^{2} \tilde{\mathbf{t}}_{k}+\tilde{\mathbf{t}}_{k} \tilde{\mathbf{e}}_{i}^{2}=\left(v+v^{-1}\right) \tilde{\mathbf{e}}_{i} \tilde{\mathbf{t}}_{k} \tilde{\mathbf{e}}_{i}, \quad \forall|i-k|=1, \\
& \tilde{\mathbf{f}}_{i}^{2} \tilde{\mathbf{t}}_{k}+\tilde{\mathbf{t}}_{k} \tilde{\mathbf{f}}_{i}^{2}=\left(v+v^{-1}\right) \tilde{\mathbf{f}}_{i} \tilde{\mathbf{t}}_{k} \tilde{\mathbf{f}}_{i}, \quad \forall|i-k|=1, \\
& \tilde{\mathbf{t}}_{k}^{2} \tilde{\mathbf{e}}_{j}+\tilde{\mathbf{e}}_{j} \tilde{\mathbf{t}}_{k}^{2}=\left(v+v^{-1}\right) \tilde{\mathbf{e}}_{j} \tilde{\mathbf{t}}_{k} \tilde{\mathbf{e}}_{j}+\tilde{\mathbf{e}}_{j}, \forall|k-j|=1, \\
& \tilde{\mathbf{t}}_{k}^{2} \tilde{\mathbf{f}}_{j}+\tilde{\mathbf{f}}_{j} \tilde{\mathbf{t}}_{k}^{2}=\left(v+v^{-1}\right) \tilde{\mathbf{f}}_{j} \tilde{\mathbf{t}}_{k} \tilde{\mathbf{f}}_{j}+\tilde{\mathbf{f}}_{j}, \forall|k-j|=1, \\
& \tilde{\mathbf{e}}_{i} \tilde{\mathbf{f}}_{j}-\tilde{\mathbf{f}}_{j} \tilde{\mathbf{e}}_{i}=\delta_{i j} \frac{\tilde{\mathbf{k}}_{i}-\tilde{\mathbf{k}}_{i}^{-1}}{v-v^{-1}} .
\end{aligned}
$$

The case for $\eta=2$ is excluded from Proposition 8.5.5 above. The algebra $\mathbf{U}_{2}^{\imath \imath}$ is generated by $\tilde{\mathbf{t}}_{0}$ and $\tilde{\mathbf{t}}_{1}$, and we have an imbedding $\imath_{d}: \mathbf{U}_{2}^{\imath \imath} \rightarrow \mathbf{U}\left(\widehat{\mathfrak{s l}_{2}}\right)$ defined by

$$
\tilde{\mathbf{t}}_{0} \mapsto \tilde{\mathbf{E}}_{0}+v \tilde{\mathbf{K}}_{0} \tilde{\mathbf{F}}_{0}+\tilde{\mathbf{K}}_{0}, \quad \tilde{\mathbf{t}}_{1} \mapsto \tilde{\mathbf{E}}_{1}+v \tilde{\mathbf{K}}_{1} \tilde{\mathbf{F}}_{1}+\tilde{\mathbf{K}}_{1}
$$

Proposition 8.5.6. The $\mathbb{Q}(v)$-algebra $\mathbf{U}_{2}^{\imath \imath}$ has a presentation with generators $\tilde{\mathbf{t}}_{0}$ and $\tilde{\mathbf{t}}_{1}$, and the following relations:

$$
\begin{aligned}
& \tilde{\mathbf{t}}_{0}^{3} \tilde{\mathbf{t}}_{1}-\llbracket 3 \rrbracket \tilde{\mathbf{t}}_{0}^{2} \tilde{\mathbf{t}}_{1} \tilde{\mathbf{t}}_{0}+\llbracket 3 \rrbracket \tilde{\mathbf{t}}_{0} \tilde{\mathbf{t}}_{1} \tilde{\mathbf{t}}_{0}^{2}-\tilde{\mathbf{t}}_{1} \tilde{\mathbf{t}}_{0}^{3}=\llbracket 2 \rrbracket^{2}\left(\tilde{\mathbf{t}}_{0} \tilde{\mathbf{t}}_{1}-\tilde{\mathbf{t}}_{1} \tilde{\mathbf{t}}_{0}\right), \\
& \tilde{\mathbf{t}}_{1}^{3} \tilde{\mathbf{t}}_{0}-\llbracket 3 \rrbracket \tilde{\mathbf{t}}_{1}^{2} \tilde{\mathbf{t}}_{0} \tilde{\mathbf{t}}_{1}+\llbracket 3 \rrbracket \tilde{\mathbf{t}}_{1} \tilde{\mathbf{t}}_{0} \tilde{\mathbf{t}}_{1}^{2}-\tilde{\mathbf{t}}_{0} \tilde{\mathbf{t}}_{1}^{3}=\llbracket 2 \rrbracket^{2}\left(\tilde{\mathbf{t}}_{1} \tilde{\mathbf{t}}_{0}-\tilde{\mathbf{t}}_{0} \tilde{\mathbf{t}}_{1}\right) .
\end{aligned}
$$


Here $\llbracket n \rrbracket=\frac{v^{n}-v^{-n}}{v-v^{-1}}$.

Proof. We first prove (8.5.2). Since $u_{2}$ is injective, it suffices to show that (8.5.2) holds in $\mathbf{U}\left(\widehat{\mathfrak{s l}_{2}}\right)$ after applying $u_{2}$. So we can assume that we are working in $\mathbf{U}\left(\widehat{\mathfrak{s l}}_{2}\right)$. Let $S\left(\tilde{\mathbf{t}}_{0}, \tilde{\mathbf{t}}_{1}\right)$ denote the term on the left-hand side of (8.5.2). Similarly, we can define $S\left(\tilde{\mathbf{t}}_{0}, \tilde{\mathbf{E}}_{1}\right)$, etc., so that we have

$$
S\left(\tilde{\mathbf{t}}_{0}, \tilde{\mathbf{t}}_{1}\right)=S\left(\tilde{\mathbf{t}}_{0}, \tilde{\mathbf{E}}_{1}\right)+S\left(\tilde{\mathbf{t}}_{0}, v \tilde{\mathbf{K}}_{1} \tilde{\mathbf{F}}_{1}\right)+S\left(\tilde{\mathbf{t}}_{0}, \tilde{\mathbf{K}}_{1}\right) .
$$

By expanding out $S\left(\tilde{\mathbf{t}}_{0}, \tilde{\mathbf{E}}_{1}\right)$, which has $4 \times 3^{4}=324$ terms in total, and using the defining relations of $\mathbf{U}\left(\widehat{\mathfrak{s l}_{2}}\right)$, we have

$$
S\left(\tilde{\mathbf{t}}_{0}, \tilde{\mathbf{E}}_{1}\right)=\llbracket 2 \rrbracket^{2}\left(\tilde{\mathbf{E}}_{0} \tilde{\mathbf{E}}_{1}-\tilde{\mathbf{E}}_{1} \tilde{\mathbf{E}}_{0}-\left(v^{2}-1\right) \tilde{\mathbf{K}}_{0} \tilde{\mathbf{E}}_{1}-\left(v^{3}-v\right) \tilde{\mathbf{K}}_{0} \tilde{\mathbf{F}}_{0} \tilde{\mathbf{E}}_{1}\right) .
$$

More precisely, the term $\tilde{\mathbf{E}}_{0} \tilde{\mathbf{E}}_{1}-\tilde{\mathbf{E}}_{1} \tilde{\mathbf{E}}_{0}$ comes from simplifying the sum of the terms in $S\left(\tilde{\mathbf{t}}_{0}, \tilde{\mathbf{E}}_{1}\right)$ involving $\tilde{\mathbf{K}}_{0} \tilde{\mathbf{F}}_{0} \tilde{\mathbf{E}}_{0}^{2} \tilde{\mathbf{E}}_{1}$ or its variants such as $\tilde{\mathbf{K}}_{0} \tilde{\mathbf{E}}_{0} \tilde{\mathbf{F}}_{0} \tilde{\mathbf{E}}_{0} \tilde{\mathbf{E}}_{1}$. The term $\tilde{\mathbf{K}}_{0} \tilde{\mathbf{E}}_{1}$ comes from simplifying the sums of $\tilde{\mathbf{K}}_{0}^{2} \tilde{\mathbf{F}}_{0} \tilde{\mathbf{E}}_{0} \tilde{\mathbf{E}}_{1}, \tilde{\mathbf{K}}_{0}^{2} \tilde{\mathbf{F}}_{0} \tilde{\mathbf{E}}_{1} \tilde{\mathbf{E}}_{0}$ and theirs variants. The term $\tilde{\mathbf{K}}_{0} \tilde{\mathbf{F}}_{0} \tilde{\mathbf{E}}_{1}$ is a result of simplifying the sums of $\tilde{\mathbf{K}}_{0}^{2} \tilde{\mathbf{F}}_{0}^{2} \tilde{\mathbf{E}}_{0} \tilde{\mathbf{E}}_{1}, \tilde{\mathbf{K}}_{0}^{2} \tilde{\mathbf{F}}_{0}^{2} \tilde{\mathbf{E}}_{1} \tilde{\mathbf{E}}_{0}$ and their variants. The rest of the terms in $S\left(\tilde{\mathbf{t}}_{0}, \tilde{\mathbf{E}}_{1}\right)$ sums to zero.

Similarly, with a very lengthy calculation as above, we obtain

$$
\begin{gathered}
S\left(\tilde{\mathbf{t}}_{0}, v \tilde{\mathbf{K}}_{1} \tilde{\mathbf{F}}_{1}\right)=\llbracket 2 \rrbracket^{2}\left(\left(v^{3}-v\right) \tilde{\mathbf{K}}_{1} \tilde{\mathbf{E}}_{0} \tilde{\mathbf{F}}_{1}+\tilde{\mathbf{K}}_{0} \tilde{\mathbf{K}}_{1}\left(\tilde{\mathbf{F}}_{0} \tilde{\mathbf{F}}_{1}-\tilde{\mathbf{F}}_{1} \tilde{\mathbf{F}}_{0}\right)+\left(v-v^{-1}\right) \tilde{\mathbf{K}}_{0} \tilde{\mathbf{K}}_{1} \tilde{\mathbf{F}}_{1}\right), \\
S\left(\tilde{\mathbf{t}}_{0}, \tilde{\mathbf{K}}_{1}\right)=\llbracket 2 \rrbracket^{2}\left(\left(v^{2}-1\right) \tilde{\mathbf{K}}_{1} \tilde{\mathbf{E}}_{0}-\left(v-v^{-1}\right) \tilde{\mathbf{K}}_{0} \tilde{\mathbf{K}}_{1} \tilde{\mathbf{F}}_{0}\right) .
\end{gathered}
$$

From (8.5.4)-(8.5.6), it is straightforward to observe that $S\left(\tilde{\mathbf{t}}_{0}, \tilde{\mathbf{t}}_{1}\right)$ is equal to the right-hand side of (8.5.2).

The equality (8.5.3) can be proved similarly. By Theorem 8.5.3 and [Ko14, Theorem 7.1], we do not need more relations for the coideal subalgebra $\mathbf{U}_{2}^{n \imath}$ of $\mathbf{U}\left(\widehat{\mathfrak{s l}}_{2}\right)$.

Remark 8.5.7. The algebra $\mathbf{U}_{2}^{\imath}$ is the so-called $q$-Onsager algebra in the literature, see [Ko14, Example 7.6] and the references therein. 
Part 3. Schur algebras and coideal subalgebras of $\mathbf{U}\left(\widehat{\mathfrak{g l}}_{n}\right)$ 


\section{CHAPTER 9}

\section{The stabilization algebra $\dot{\mathbf{K}}_{n}^{\mathfrak{c}}$ arising from Schur algebras}

In this chapter we study the stabilization of the family of Schur algebras $\mathbf{S}_{n, d}^{\mathfrak{c}}$ (as $d$ varies), which leads to the formulation of the stabilization algebra $\dot{\mathbf{K}}_{n}^{\mathfrak{c}}$ as well as its monomial and stably canonical bases. One difficulty of working with the Schur algebra $\mathbf{S}_{n, d}^{\mathfrak{c}}$ directly is that it does not have a good generating set. We overcome the difficulty by embedding $\mathbf{S}_{n, d}^{\mathfrak{c}}$ into a Lusztig algebra of higher rank. This allows us to understand monomial bases, multiplication, comultiplication and bar operators of the Schur algebras and their stabilization properties in a conceptual way and lift these structures to $\dot{\mathbf{K}}_{n}^{\mathfrak{c}}$. We show that the pair $\left(\dot{\mathbf{K}}_{n}, \dot{\mathbf{K}}_{n}^{\mathfrak{c}}\right)$ forms a quantum symmetric pair in an idempotented form, where $\dot{\mathbf{K}}_{n}$ is isomorphic to the idempotented quantum affine $\mathfrak{g l}_{n}$.

\subsection{Monomial BASES For SCHur Algebras}

Recall $n=2 r+2$ for $r \geqslant 0$. We set

$$
\breve{r}=r+1, \quad \breve{n}=2 \breve{r}+2 .
$$

We consider the subset $\Xi_{\breve{n}, d}^{J \jmath}$ which consists of all matrices $A \in \Xi_{\breve{n}, d}$ such that $a_{1, j}=a_{i, 1}=0$ for all $i, j \in \mathbb{Z}$. Then the deleting operator $\operatorname{dlt}_{1}$ of the row and column $\pm 1 \bmod \breve{n}$ defines

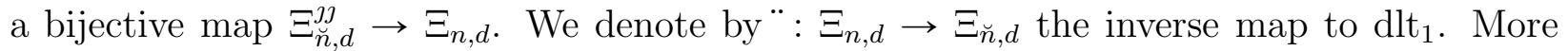
generally, we may regard " as an imbedding

$$
\cdot: \Xi_{n, d} \longrightarrow \Xi_{\breve{n}, d}, \quad A \mapsto \ddot{A},
$$

by adding suitable rows and columns of zeros.

As we will study the behavior of the various bases in $\mathbf{S}_{n, d}^{\mathfrak{c}}$ and $\mathbf{S}_{\breve{n}, d}$ under stabilization, we shall put a subscript $d$ to emphasize the dependence of $d$, e.g., $[A]_{d}$.

Just like our study of $\imath \jmath, \jmath$ and $\imath$ versions, we consider the following idempotent in $\mathbf{S}_{\breve{n}, d}^{\mathfrak{c}}$ and its associated subalgebra:

$$
\ddot{\mathbf{S}}_{n, d}^{\mathfrak{c}}=\breve{\mathbf{j}}_{1} \mathbf{S}_{\breve{n}, d}^{\mathfrak{c}} \breve{\mathbf{j}}_{1}, \quad \text { where } \quad \breve{\mathbf{j}}_{1}=\sum_{A \in \Xi_{n, d}: A \text { diagonal }}[\ddot{A}]_{d} .
$$

Proposition 9.1.1. There is an algebra imbedding $\rho: \mathbf{S}_{n, d}^{\mathfrak{c}} \rightarrow \mathbf{S}_{\breve{n}, d}^{\mathfrak{c}},[A]_{d} \mapsto[\ddot{A}]_{d}$, for $A \in \Xi_{n, d}$, and an induced algebra isomorphism $\rho: \mathbf{S}_{n, d}^{\mathfrak{c}} \stackrel{\simeq}{\longrightarrow} \ddot{\mathbf{S}}_{n, d}^{\mathfrak{c}}$, which are compatible with the canonical bases.

Proof. We define an imbedding $\mathcal{X}_{n, d}^{\mathfrak{c}} \rightarrow \mathcal{X}_{\breve{n}, d}^{\mathfrak{c}}, L \mapsto \breve{L}$ by adding to $L$ an extra copy of $L_{1}$ and $L_{-2} \bmod n$. Specifically, the lattice chains from 0 to $\breve{n}$ in $\breve{L}$ are

$$
\left(L_{0}, L_{0}, L_{1}, \cdots, L_{n-1}, L_{n-1}, L_{n}\right) \text {. }
$$


This imbedding clearly induces an injective algebra homomorphism $\mathbf{S}_{n, d}^{\mathfrak{c}} \rightarrow \mathbf{S}_{\breve{n}, d}^{\mathfrak{c}}$, with image being $\ddot{\mathbf{S}}_{n, d}^{\mathfrak{c}}$.

By Proposition 9.1.1, we can study Schur algebra $\mathbf{S}_{n, d}^{\mathfrak{c}}$ through $\ddot{\mathbf{S}}_{n, d}^{\mathfrak{c}}$, which has an advantage that it admits an inclusion

$$
\ddot{\mathbf{S}}_{n, d}^{\mathfrak{c}} \subseteq \mathbf{U}_{\breve{n}, d}^{\mathfrak{c}},
$$

since $\ddot{\mathbf{S}}_{n, d}^{\mathfrak{c}}$ is spanned by canonical basis elements parametrized by matrices whose second columns are zero; such matrices are automatically aperiodic. Hence we have

$$
\rho: \mathbf{S}_{n, d}^{\mathfrak{c}} \longrightarrow \mathbf{U}_{\breve{n}, d}^{\mathfrak{c}}
$$

The pair $\left(\mathbf{S}_{n, d}^{\mathfrak{c}}, \mathbf{U}_{\breve{n}, d}^{\mathfrak{c}}\right)$ for $\mathbf{S}_{n, d}^{\mathfrak{c}}$ plays a similar role as what the pairs $\left(\mathbf{S}_{\mathfrak{n}, d}^{\jmath \imath}, \mathbf{S}_{n, d}^{\mathfrak{c}}\right),\left(\mathbf{S}_{\mathfrak{n}, d}^{\imath \jmath}, \mathbf{S}_{n, d}^{\mathfrak{c}}\right)$ and $\left(\mathbf{S}_{\eta, d}^{\imath \imath}, \mathbf{S}_{n, d}^{\mathfrak{c}}\right)$ do for $\mathbf{S}_{\mathfrak{n}, d}^{\jmath \imath}, \mathbf{S}_{\mathfrak{n}, d}^{\imath \jmath}$, and $\mathbf{S}_{\eta, d}^{\imath \imath}$, respectively.

We shall put a superscript ${ }^{\triangleleft}$ on the Chevalley generators of $\mathbf{S}_{\breve{n}, d}^{\mathfrak{c}}$. For convenience, let $\breve{\mathbf{f}}_{i}=\breve{\mathbf{e}}_{\breve{n}-(i+1)}$ if $r+1 \leqslant i \leqslant \breve{n}-1$ and $\breve{\mathbf{f}}_{i}=\breve{\mathbf{f}}_{\breve{n}+i}$ for all $i \in \mathbb{Z}$. To each tridiagonal matrix $A \in \Xi_{n, d}$ such that $A-\sum_{1 \leqslant i \leqslant n} \alpha_{i} E_{\theta, n}^{i, i+1}$ is diagonal, we set $\alpha_{0}=\alpha_{n}$ and

$$
\ddot{\mathbf{f}}_{A ; d}=\breve{\mathbf{f}}_{0}^{\left(\alpha_{0}\right)} * \breve{\mathbf{f}}_{n}^{\left(\alpha_{n-1}\right)} * \breve{\mathbf{f}}_{n+1}^{\left(\alpha_{n-1}\right)} *\left(\breve{\mathbf{f}}_{n-1}^{\left(\alpha_{n-2}\right)} * \breve{\mathbf{f}}_{n-2}^{\left(\alpha_{n-3}\right)} * \cdots * \breve{\mathbf{f}}_{1}^{\left(\alpha_{0}\right)}\right) * 1_{\operatorname{co}(\ddot{A})},
$$

where the idempotent $1_{\operatorname{co}(\ddot{A})}$ is the standard basis element attached to the diagonal matrix in $\Xi_{\breve{n}, d}$ with diagonal $\operatorname{co}(\ddot{A})$. Note that the product is taken in $\mathbf{S}_{\breve{n}, d}^{\mathfrak{c}}$. Since it lies in the component $\mathbf{S}_{\breve{n}, d}^{\mathfrak{c}}(\operatorname{ro}(\ddot{A}), \operatorname{co}(\ddot{A}))$ and hence lies in the image of $\rho$, we can define an element $\mathbf{f}_{A ; d}$ in $\mathbf{S}_{n, d}^{\mathfrak{c}}$ to be its preimage under $\rho$, i.e.,

$$
\mathbf{f}_{A ; d}=\rho^{-1}\left(\ddot{\mathbf{f}}_{A ; d}\right)
$$

Lemma 9.1.2. For each tridiagonal matrix $A$ in $\Xi_{n, d}$, we have $[A]_{d}=\mathbf{f}_{A ; d}+$ lower terms.

Proof. It is reduced to showing a similar statement for $\ddot{\mathbf{f}}_{A ; d}$ in $\mathbf{S}_{\breve{n}, d}^{\mathfrak{c}}$ via $\rho$. We first observe that the monomial $\breve{\mathbf{f}}_{n+1}^{\left(\alpha_{n-1}\right)} *\left(\breve{\mathbf{f}}_{n-1}^{\left(\alpha_{n-2}\right)} * \breve{\mathbf{f}}_{n-2}^{\left(\alpha_{n-3}\right)} * \cdots * \breve{\mathbf{f}}_{1}^{\left(\alpha_{0}\right)}\right) * 1_{\operatorname{co}(\ddot{A})}$ (a part of (9.1.4)) has a leading term $\left[A^{\prime}\right]_{d}$ of a certain tridiagonal matrix $A^{\prime}$ such that $A^{\prime}-\sum_{1 \leqslant i \leqslant n-1} \alpha_{i-1} E_{\theta, \breve{n}}^{i, i+1}-\alpha_{n-1} E_{\theta, \breve{n}}^{n+1, n+2}$ is diagonal. In particular, the off-diagonal upper triangular entries of $A^{\prime}$ are the same as those of $\ddot{A}$ except at $(0,2),(1,2),(n, n+2),(n+1, n+2) \bmod \breve{n}$. After composing with $\breve{\mathbf{f}}_{0}^{\left(\alpha_{0}\right)} * \breve{\mathbf{f}}_{n}^{\left(\alpha_{n-1}\right)}$ and using Lemma 4.4.1, we see that the leading term of $\ddot{\mathbf{f}}_{A ; d}$ is exactly $[\ddot{A}]_{d}$. Transporting back via $\rho^{-1}$, the lemma is thus proved.

As a product of bar-invariant Chevalley generators in $\mathbf{S}_{\breve{n}, d}^{\mathfrak{c}}, \ddot{\mathbf{f}}_{A ; d}$ is bar invariant in $\mathbf{S}_{\breve{n}, d}^{\mathfrak{c}}$. Since the imbedding $\rho$ is compatible with the bar operators in $\mathbf{S}_{n, d}^{\mathfrak{c}}$ and $\mathbf{S}_{\breve{n}, d}^{\mathfrak{c}}$, the preimage $\rho^{-1}\left(\mathbf{f}_{A ; d}\right)$ must be bar invariant in $\mathbf{S}_{n, d}^{\mathfrak{c}}$. Thus we have the following.

Lemma 9.1.3. One has $\overline{\mathbf{f}_{A ; d}}=\mathbf{f}_{A ; d}$ for all tridiagonal $A \in \Xi_{n, d}$.

To a matrix, we define the depth of $A$ by

$$
\operatorname{dep}(A)=\max \left\{l \in \mathbb{N} \mid a_{i, i+l} \neq 0 \text { for some } i\right\} .
$$


The following description of leading terms leads to the determination of a set of multiplicative generators for the Schur algebra $\mathbf{S}_{n, d}^{\mathfrak{c}}$.

Proposition 9.1.4. Let $A, B \in \Xi_{n, d}$ such that $\operatorname{ro}(A)=\operatorname{co}(B)$ and $\operatorname{dep}(A) \leqslant m$ for some positive integer $m$. Assume further that $B-\sum_{1 \leqslant i \leqslant n} \beta_{i} E_{\theta}^{i, i+1}$ is diagonal for some $\beta \in \mathbb{Z}_{n}$ and $a_{i+1, i+m} \geqslant \beta_{i} \geqslant 0$ for all $i$. Then we have

$$
[B]_{d} *[A]_{d}=\left[A+\sum_{1 \leqslant i \leqslant n} \beta_{i}\left(E_{\theta}^{i, i+m}-E_{\theta}^{i+1, i+m}\right)\right]_{d}+\text { lower terms. }
$$

Proof. It is enough to show a similar statement with $[B]_{d}$ replaced by $\mathbf{f}_{B ; d}$ by Lemma 9.1.2, We then transport this problem to the setting of $\mathbf{S}_{\breve{n}, d}^{\mathfrak{c}}$ and use Lemma 4.4.2. Now the order in (9.1.4) allows us to push $\beta_{0}$ and $\beta_{n-1}$ across rows 1 and $n+1$ respectively to the desired positions. The statement then follows by pulling back to $\mathbf{S}_{n, d}^{\mathfrak{c}}$ via $\rho$.

Let us present an example explaining the proof of Proposition 9.1.4.

Example 9.1.5. Let $A$ be the following matrix in $\Xi_{4, d}$ with $n=4$ and $\breve{n}=6$.

\begin{tabular}{c|c|c|c|c|c|c|c|c|c|c|c} 
& $\mathrm{c}-3$ & $\mathrm{c}-2$ & $\mathrm{c}-1$ & $\mathrm{c} 0$ & $\mathrm{c} 1$ & $\mathrm{c} 2$ & $\mathrm{c} 3$ & $\mathrm{c} 4$ & $\mathrm{c} 5$ & $\mathrm{c} 6$ & $\mathrm{c} 7$ \\
\hline & & & & & & & & & & & \\
\hline $\mathrm{r} 0$ & & 7 & $*$ & $d_{0}$ & $*$ & 7 & & & & & \\
\hline $\mathrm{r} 1$ & & & 6 & $*$ & $d_{1}$ & $*$ & 5 & & & & \\
\hline $\mathrm{r} 2$ & & & & 4 & $*$ & $d_{2}$ & $*$ & 4 & & & \\
\hline $\mathrm{r} 3$ & & & & & 5 & $*$ & $d_{3}$ & $*$ & 6 & & \\
\hline $\mathrm{r} 4$ & & & & & & 7 & $*$ & $d_{0}$ & $*$ & 7 & \\
\hline & & & & & & & & & & &
\end{tabular}

where $d_{i}$ is the diagonal entries of $A$ and $*$ are some nonnegative integers irrelevant to the discussion. Now let $\left(\beta_{i}\right)_{1 \leqslant i \leqslant 4}=(2,4,5,3)$ such that $B-\sum_{1 \leqslant i \leqslant 4} \beta_{i} E_{\theta}^{i, i+1}$ is diagonal and $\operatorname{ro}(A)=\operatorname{co}(B)$. We want to determine the leading term of $\mathbf{f}_{B ; d} *[A]_{d}$. By definition, we have

$$
\ddot{\mathbf{f}}_{B ; d}=\breve{\mathbf{f}}_{0}^{(3)} * \breve{\mathbf{f}}_{4}^{(5)} * \breve{\mathbf{f}}_{5}^{(5)} * \breve{\mathbf{f}}_{3}^{(4)} * \breve{\mathbf{f}}_{2}^{(2)} * \breve{\mathbf{f}}_{1}^{(3)} * 1_{\operatorname{co}(\ddot{B})}
$$

Now we expand $A$ at row/column \pm 1 to get the matrix $\ddot{A}$ in $\Xi_{6, d}$, which is completely determined by its upper triangular part as follows.

\begin{tabular}{c|c|c|c|c|c|c|c|c|c|c|c} 
& $\mathrm{c}-1$ & $\mathrm{c} 0$ & $\mathrm{c} 1$ & $\mathrm{c} 2$ & $\mathrm{c} 3$ & $\mathrm{c} 4$ & $\mathrm{c} 5$ & $\mathrm{c} 6$ & $\mathrm{c} 7$ & $\mathrm{c} 8$ & $\mathrm{c} 9$ \\
\hline & & & & & & & & & & & \\
\hline $\mathrm{r} 0$ & & $d_{0}$ & 0 & $*$ & 7 & & & & & & \\
\hline $\mathrm{r} 1$ & & & 0 & 0 & 0 & 0 & 0 & & & & \\
\hline $\mathrm{r} 2$ & & & & $d_{1}$ & $*$ & 5 & 0 & & & & \\
\hline $\mathrm{r} 3$ & & & & & $d_{2}$ & $*$ & 0 & 4 & & & \\
\hline $\mathrm{r} 4$ & & & & & & $d_{3}$ & 0 & $*$ & 0 & 6 & \\
\hline $\mathrm{r} 5$ & & & & & & & 0 & 0 & 0 & 0 & \\
\hline $\mathrm{r} 6$ & & & & & & & & $d_{0}$ & 0 & $*$ & 7 \\
\hline & & & & & & & & & & &
\end{tabular}


Then we apply $\ddot{\mathbf{f}}_{B ; d}$ to $[\ddot{A}]_{d}$ to get the following leading term.

\begin{tabular}{l|l|l|l|l|c|c|c|c|c|c|c|c|c} 
& $\mathrm{c}-1$ & $\mathrm{c} 0$ & $\mathrm{c} 1$ & $\mathrm{c} 2$ & $\mathrm{c} 3$ & $\mathrm{c} 4$ & $\mathrm{c} 5$ & $\mathrm{c} 6$ & $\mathrm{c} 7$ & $\mathrm{c} 8$ & $\mathrm{c} 9$ & $\mathrm{c} 10$ & $\mathrm{c} 11$ \\
\hline & & & & & & & & & & & & & \\
\hline $\mathrm{r} 0$ & & $d_{0}$ & 0 & 2 & 3 & & & & & & & & \\
\hline $\mathrm{r} 1$ & & & 0 & 0 & 0 & 0 & & & & & & & \\
\hline $\mathrm{r} 2$ & & & & $d_{1}$ & $*$ & 2 & 0 & 2 & & & & & \\
\hline $\mathrm{r} 3$ & & & & & $d_{2}$ & $*$ & 0 & 2 & 0 & 4 & & & \\
\hline $\mathrm{r} 4$ & & & & & & $d_{3}$ & 0 & $*$ & 0 & 2 & 5 & & \\
\hline $\mathrm{r} 5$ & & & & & & & 0 & 0 & 0 & 0 & 0 & & \\
\hline $\mathrm{r} 6$ & & & & & & & & $d_{0}$ & 0 & $*$ & 2 & 3 & \\
\hline & & & & & & & & & & & & &
\end{tabular}

This leading term is corresponding to the expected matrix in $\Xi_{4, d}$ whose upper triangular part is as follows.

\begin{tabular}{c|c|c|c|c|c|c|c|c|c} 
& $\mathrm{c}-1$ & $\mathrm{c} 0$ & $\mathrm{c} 1$ & $\mathrm{c} 2$ & $\mathrm{c} 3$ & $\mathrm{c} 4$ & $\mathrm{c} 5$ & $\mathrm{c} 6$ & $\mathrm{c} 7$ \\
\hline & & & & & & & & & \\
\hline $\mathrm{r} 0$ & & $d_{0}$ & $*$ & 2 & 3 & & & & \\
\hline $\mathrm{r} 1$ & & & $d_{1}$ & $*$ & 2 & 2 & & & \\
\hline $\mathrm{r} 2$ & & & & $d_{2}$ & $*$ & 2 & 4 & & \\
\hline $\mathrm{r} 3$ & & & & & $d_{3}$ & $*$ & 2 & 5 & \\
\hline $\mathrm{r} 4$ & & & & & & $d_{0}$ & $*$ & 2 & 3 \\
\hline & & & & & & & & &
\end{tabular}

The following theorem is obtained by applying Proposition 9.1.4 repeatedly.

Theorem 9.1.6. For any matrix $A=\left(a_{i j}\right) \in \Xi_{n, d}$ of depth $m$, there exist unique tridiagonal matrices $A_{1}, A_{2}, \ldots, A_{m} \in \Xi_{n, d}$ satisfying $\operatorname{ro}\left(A_{m}\right)=\operatorname{ro}(A), \operatorname{co}\left(A_{1}\right)=\operatorname{co}(A), \operatorname{ro}\left(A_{i}\right)=$ $\operatorname{co}\left(A_{i+1}\right)$ for $1 \leqslant i \leqslant m-1$ and $A_{i}-\sum_{1 \leqslant j \leqslant n}\left(\sum_{k \leqslant j-i+1} a_{k, j+1}\right) E_{\theta}^{j, j+1}$ is diagonal for all $1 \leqslant i \leqslant m$ such that the following formulas hold in $\mathbf{S}_{n, d}^{\mathfrak{c}}$ :

$$
\begin{aligned}
{\left[A_{m}\right]_{d} *\left[A_{m-1}\right]_{d} * \cdots *\left[A_{1}\right]_{d} } & =[A]_{d}+\text { lower terms }, \\
\mathbf{f}_{A ; d}:=\mathbf{f}_{A_{m} ; d} * \mathbf{f}_{A_{m-1} ; d} * \cdots * \mathbf{f}_{A_{1} ; d} & =[A]_{d}+\text { lower terms. }
\end{aligned}
$$

Proof. We prove (9.1.7) by induction with respect to the $\operatorname{depth}$ of $A$. If $\operatorname{dep}(A)=0$, the matrix $A$ is diagonal, and the statement is clearly true. Now assume that $\operatorname{dep}(A)=m>0$ and the statement holds for all matrices of depth $<m$. Set

$$
A^{\prime}=A-\sum_{1 \leqslant i \leqslant n} a_{i, i+m}\left(E_{\theta}^{i, i+m}-E_{\theta}^{i+1, i+m}\right) .
$$

Let $B$ be the unique tridiagonal matrix in $\Xi_{n, d}$ such that $B-\sum_{1 \leqslant i \leqslant n} a_{i, i+m} E_{\theta}^{i, i+1}$ is diagonal and $\operatorname{co}(B)=\operatorname{ro}\left(A^{\prime}\right)$. By Proposition 9.1 .4 , we have $[B]_{d} *\left[A^{\prime}\right]_{d}=[A]_{d}+$ lower terms. Now observing that $\operatorname{dep}\left(A^{\prime}\right)<m$, we complete the proof of (9.1.7) by induction.

The second formula (9.1.8) for $\mathbf{f}_{A ; d}$ follows from (9.1.7) and Lemma 9.1.2.

Corollary 9.1.7. The set $\left\{\mathbf{f}_{A ; d} \mid A \in \Xi_{n, d}\right\}$ forms a basis for $\mathbf{S}_{n, d}^{\mathfrak{c}}$ (called a monomial basis).

Corollary 9.1.8. The set $\left\{\mathbf{f}_{A ; d} \mid A \in \Xi_{n, d}\right.$ tridiagonal $\}$ (respectively, $\left\{[A]_{d} \mid A \in \Xi_{n, d}\right.$ tridiagonal $\}$ ) forms a generating set for the algebra $\mathbf{S}_{n, d}^{\mathfrak{c}}$. 


\subsection{Stabilization of the Schur algebras}

In this section, we study the stabilization of the multiplication and bar operator of the Schur algebras $\mathbf{S}_{n, d}^{\mathfrak{c}}$.

Recall that $I_{n}=\sum_{1 \leqslant i \leqslant n} E_{n}^{i i}$. Recall the operation “ from (9.1.1) so that $\ddot{I}_{n}=I_{\breve{n}}-E_{\theta, \breve{n}}^{1,1}$. We set

$$
\ddot{p} A=A+p \ddot{I}_{n}, \quad \forall A \in \widetilde{\Xi}_{\breve{n}} .
$$

Introduce the algebra $\mathscr{R}=\mathbb{Q}(v)\left[v^{\prime}, v^{\prime-1}\right]$ with a bar involution such that $\bar{v}=v^{-1}$ and $\overline{v^{\prime}}=v^{\prime-1}$. For $a \in \mathbb{Z}$ and $b \in \mathbb{N}$, we define the following polynomials in $\mathscr{R}$ :

$$
\left[\begin{array}{l}
a \\
b
\end{array}\right]_{v, v^{\prime}}=\prod_{1 \leqslant i \leqslant b} \frac{v^{2(a-i+1)} v^{\prime-2}-1}{v^{2 i}-1}, \quad \text { and } \quad[a]_{v, v^{\prime}}=\left[\begin{array}{l}
a \\
1
\end{array}\right]_{v, v^{\prime}} .
$$

For $0 \leqslant i \leqslant \breve{n}-1, A \in \widetilde{\Xi}_{\breve{n}, d}$ with $a_{1, j}=0$ for all $j \in \mathbb{Z}$ and $t=\left(t_{u}\right)_{u \in \mathbb{Z}} \in \mathbb{N}^{\mathbb{Z}}$ such that $\sum_{j \in \mathbb{Z}} t_{u}=$ $R$, we define a polynomial $Q_{i, R ; A}^{t}\left(v, v^{\prime}\right)$ in $\mathscr{R}$ as follows. For any $i \in[0, \breve{n}-1] \backslash\{0, \breve{r}+1,1, n+1\}$, we define

$$
Q_{i, R ; A}^{t}\left(v, v^{\prime}\right)=v^{\beta_{t}} \prod_{u \in \mathbb{Z}, u \neq i} \overline{\left[\begin{array}{c}
a_{i u}+t_{u} \\
t_{u}
\end{array}\right]} \cdot v^{\prime\left(\delta_{i, 1}+\delta_{i, \breve{n}-1}\right) \sum_{i+1 \geqslant u} t_{u}} \overline{\left[\begin{array}{c}
a_{i i}+t_{i} \\
t_{i}
\end{array}\right]_{v, v^{\prime}}},
$$

where

$$
\beta_{t}=\sum_{j \geqslant u} a_{i j} t_{u}-\sum_{j>u} a_{i+1, j} t_{u}+\sum_{j<u} t_{j} t_{u}+\frac{1}{2}\left(\delta_{i, \breve{r}}+\delta_{i, \breve{n}-1}\right)\left(\sum_{j+u<2(i+1)} t_{j} t_{u}+\sum_{j<i+1} t_{j}\right) .
$$

For $i=1$ or $n+1$, we define

$$
Q_{i, R ; A}^{t}\left(v, v^{\prime}\right)=v^{\beta_{t}} \prod_{u \in \mathbb{Z}, u \neq i} \overline{\left[\begin{array}{c}
a_{i u}+t_{u} \\
t_{u}
\end{array}\right]} \cdot v^{-\sum_{i \geqslant u} t_{u}} .
$$

For $i=0$ or $\breve{r}+1$, we define

$$
Q_{i, R ; A}^{t}\left(v, v^{\prime}\right)=v^{\beta_{t}^{\prime}} \prod_{u>i} \overline{\left[\begin{array}{c}
a_{i u}+t_{u}+t_{2 i-u} \\
t_{u}
\end{array}\right]} \prod_{u<i} \overline{\left[\begin{array}{c}
a_{i u}+t_{u} \\
t_{u}
\end{array}\right]} \cdot \prod_{u=0}^{t_{i}-1} \frac{\overline{\left[a_{i i}+1+2 u\right]_{v, v^{\prime}}}}{\overline{[u+1]}},
$$

where

$$
\beta_{t}^{\prime}=\sum_{j \geqslant u} a_{i j} t_{u}-\sum_{j>u} a_{i+1, j} t_{u}+\sum_{j<u, j+u \leqslant 2 i} t_{j} t_{u}-\sum_{j>i} \frac{t_{j}^{2}-t_{j}}{2}+\frac{R^{2}-R}{2} .
$$

The following lemma follows directly from the definition.

Lemma 9.2.1. We have $Q_{i, R ;{ }_{p} A}^{t}(v, 1)=Q_{i, R ; A}^{t}\left(v, v^{-p}\right)$, for all $p \in 2 \mathbb{Z}$ and all admissible $i, t, R, A$.

Given the same data $(i, A, t)$ as above, we define

$$
A_{i, R, t}=A+\sum_{u \in \mathbb{Z}} t_{u}\left(E_{\theta, \breve{n}}^{i, u}-E_{\theta, \breve{n}}^{i+1, u}\right) .
$$

It is convenient to introduce the following notations for later use.

$$
\mathbf{f}_{A ; d}=[A]_{d}=0, \quad \forall A \notin \Xi_{n, d} .
$$


The following lemma describes the stabilization behavior of the multiplication formulas in $\mathbf{S}_{\breve{n}, d}^{\mathfrak{c}}$ after adding $p \ddot{I}_{n}$.

Lemma 9.2.2. Assume $A, B \in \widetilde{\Xi}_{\breve{n}, d}$ and $R \in \mathbb{N}$ satisfy the following properties: $\operatorname{ro}(A)=$ $\operatorname{co}(B), B-R E_{\theta, \breve{n}}^{i, i+1}$ is diagonal for some $1 \leqslant i \leqslant \breve{n}$ and $a_{1, j}=0$ for all $j \in \mathbb{Z}$. Then we have

$$
[\ddot{p} B]_{d+\frac{p}{2} n} *[\ddot{p} A]_{d+\frac{p}{2} n}=\sum_{t} Q_{i, R ; A}^{t}\left(v, v^{-p}\right)\left[\ddot{p} A_{i, R, t}\right]_{d+\frac{p}{2} n}, \quad \forall p \in 2 \mathbb{Z} .
$$

where the sum runs over all sequences $t=\left(t_{u}\right) \in \mathbb{N}^{\mathbb{Z}}$ such that $\sum_{u \in \mathbb{Z}} t_{u}=R$ and $A_{i, R, t} \in \widetilde{\Xi}_{\breve{n}, d}$, independent of $p$.

Proof. We observe that the specialization $Q_{i, R ; A}^{t}(v, 1)$ of $Q_{i, R ; A}^{t}\left(v, v^{\prime}\right)$ at $v^{\prime}=1$ is exactly the structure constant of $\left[A_{i, R, t}\right]_{d}$ in the multiplication formulas in Proposition 4.3.3, modulo some changes of indexes for $\breve{r}+1 \leqslant i \leqslant \breve{n}-1$. The lemma follows then from Lemma 9.2.1 and the convention (9.2.5).

We shall need a stronger version of Lemma 9.2.2, Given tuples $\mathbf{i}=\left(i_{1}, \ldots, i_{s}\right)$ and $\mathbf{a}=$ $\left(a_{1}, \ldots, a_{s}\right) \in \mathbb{N}^{s}$, we introduce the notation

$$
\mathbf{i}_{\geqslant l}=\left(i_{l}, i_{l+1}, \ldots, i_{s}\right), \quad \mathbf{a}_{\geqslant l}=\left(a_{l}, a_{l+1}, \ldots, a_{s}\right), \quad \forall 1 \leqslant l \leqslant s .
$$

Given a tuple $\mathbf{t}=\left(t_{1}, \ldots, t_{s}\right)$ of sequences such that

$$
\text { the } l \text {-th component } t_{l}=\left(t_{l, j}\right)_{j \in \mathbb{Z}} \in \mathbb{N}^{\mathbb{Z}} \text { satisfies } \sum_{j \in \mathbb{Z}} t_{l, j}=a_{l} \text { for all } 1 \leqslant l \leqslant s
$$

and a matrix $A \in \widetilde{\Xi}_{\breve{n}, d}$ such that $a_{1, j}=0$ for all $j \in \mathbb{Z}$, we define inductively the matrix $A_{\mathbf{i}, \mathbf{a}, \mathbf{t}}$ and the polynomial $Q_{\mathbf{i}, \mathbf{a} ; A}^{\mathbf{t}}\left(v, v^{\prime}\right)$ in $\mathscr{R}$ via (9.2.4) as follows:

$$
\begin{aligned}
A_{\mathbf{i}, \mathbf{a}, \mathbf{t}} & =\left(A_{\mathbf{i}_{\geqslant 2}, \mathbf{a} \geqslant 2, \mathbf{t}_{\geqslant 2}}\right)_{i_{1}, a_{1}, t_{1}}, \\
Q_{\mathbf{i}, \mathbf{a} ; A}^{\mathbf{t}}\left(v, v^{\prime}\right) & =Q_{i_{1}, a_{1} ; A_{\mathbf{i}_{\geqslant}, \mathbf{a} \geqslant 2, \mathbf{t}_{\geqslant 2}}}^{t_{1}}\left(v, v^{\prime}\right) \cdot Q_{\mathbf{i}_{\geqslant 2}, \mathbf{a} \geqslant 2 ; A}^{\mathbf{t} \geqslant 2}\left(v, v^{\prime}\right) .
\end{aligned}
$$

By Lemma 9.2.1 and by induction on the length of $\mathbf{i}$, we have

$$
Q_{\mathbf{i}, \mathbf{a} ; A}^{\mathbf{t}}\left(v, v^{-p}\right)=Q_{\mathbf{i}, \mathbf{a} ; \ddot{p} A}^{\mathbf{t} A}(v, 1)
$$

Given a pair $(\mathbf{i}, \mathbf{a})$ and $A \in \widetilde{\Xi}_{\breve{n}, d}$ such that $a_{1, j}=0$ for all $j \in \mathbb{Z}$, we define the set $\mathcal{T}_{\mathbf{i}, \mathbf{a}, A}$ to be the set of all tuples $\mathbf{t}=\left(t_{1}, \ldots, t_{s}\right)$ of sequences in $\mathbb{N}^{\mathbb{Z}}$ such that the $l$-th component $t_{l}=\left(t_{l, j}\right)_{j \in \mathbb{Z}}$ satisfies $\sum_{j \in \mathbb{Z}} t_{l, j}=a_{j}$ for all $1 \leqslant j \leqslant s, A_{\mathbf{i}_{\geqslant l}, \mathbf{a} \geqslant l, \mathbf{t}_{\geqslant l}} \in \widetilde{\Xi}_{\breve{n}, d}$ for all $1 \leqslant l \leqslant s$. Clearly, we have $\mathcal{T}_{\mathbf{i}, \mathbf{a}, A}=\mathcal{T}_{\mathbf{i}, \mathbf{a}, \ddot{p} A}$ for all $p$.

Proposition 9.2.3. Assume $A, B_{j} \in \widetilde{\Xi}_{\breve{n}, d}$, for all $1 \leqslant j \leqslant s$ and pairs of tuples (i,a) satisfy the following properties: $\operatorname{ro}(A)=\operatorname{co}\left(B_{s}\right), \operatorname{ro}\left(B_{u}\right)=\operatorname{co}\left(B_{u-1}\right), \forall 1<u \leqslant s, B_{u}-a_{u} E_{\theta, \grave{n}}^{i_{u}, i_{u}+1}$ is diagonal for $1 \leqslant u \leqslant s$, and $a_{1, u}=0$ for all $j \in \mathbb{Z}$. Then we have

$$
\left[\ddot{p} B_{1}\right]_{d+\frac{p}{2} n} * \cdots *\left[\ddot{p} B_{s}\right]_{d+\frac{p}{2} n} *[\ddot{p} A]_{d+\frac{p}{2} n}=\sum_{\mathbf{t} \in \mathcal{T}_{\mathbf{i}, \mathbf{a}, A}} Q_{\mathbf{i}, \mathbf{a} ; A}^{\mathbf{t}}\left(v, v^{-p}\right)\left[\ddot{p} A_{\mathbf{i}, \mathbf{a}, \mathbf{t}}\right]_{d+\frac{p}{2} n}, \quad \forall p \in 2 \mathbb{Z} .
$$


Proof. Let $\mathcal{T}_{\mathbf{i}, \mathbf{a}, A ; d}$ be the subset of $\mathcal{T}_{\mathbf{i}, \mathbf{a}, A}$ consisting of all $\mathbf{t}$ such that $A_{\mathbf{i}_{\geqslant l}, \mathbf{a} \geqslant l, \mathbf{t}_{\geqslant l}} \in \Xi_{\breve{n}, d}$ for all $1 \leqslant l \leqslant s$, where $s$ is the length of $\mathbf{i}$. In view of Lemma 9.2.2, the left-hand side of the equality in the lemma is equal to

$$
\sum_{\mathbf{t} \in \mathcal{T}_{\mathbf{i}, \mathbf{a}, A ; d+p n}} Q_{\mathbf{i}, \mathbf{a} ; A}^{\mathbf{t}}\left(v, v^{-p}\right)\left[\ddot{p} A_{\mathbf{i}, \mathbf{a}, \mathbf{t}}\right]_{d+\frac{p}{2} n} .
$$

It is reduced to showing that if $\ddot{p} A_{\mathbf{i}, \mathbf{a}, \mathbf{t}} \in \Xi_{\breve{n}, d+\frac{p}{2} n}$ and $\ddot{p}_{\mathbf{i}_{\geqslant l}, \mathbf{a} \geqslant l, \mathbf{t}_{\geqslant l}} \notin \Xi_{\breve{n}, d+\frac{p}{2} n}$ for some $l$, then the structure constant of $\left[\ddot{p} A_{\mathbf{i}, \mathbf{a}, \mathbf{t}}\right]_{d+\frac{p}{2} n}$ is zero. In such a case, there is an $l_{0}$ such that $\ddot{p} A_{\mathbf{i}_{\geqslant}, l_{0}, \mathbf{a}_{\geqslant}, l_{0}, \mathbf{t}_{\geqslant l_{0}}} \notin \Xi_{\breve{n}, d+\frac{p}{2} n}$ and $\ddot{p}_{\mathbf{i}_{\geqslant l_{0}+1}, \mathbf{a}_{\geqslant} l_{0}+1}, \mathbf{t}_{\geqslant l_{0}+1} \in \Xi_{\breve{n}, d+\frac{p}{2} n}$; this implies that the $i_{l_{0}+1^{-}}$th diagonal entry of $\ddot{p} A_{\mathbf{i}_{\geqslant l_{0}}, \mathbf{a} \geqslant l_{0}}, \mathbf{t}_{\geqslant l_{0}}$ is negative, while nonnegative after adding the $i_{l_{0}+1}$-th entry of the tuple $t_{l_{0}+1}$. The latter condition further yields that the factor $Q_{i_{l_{0}+1}, a_{l_{0}+1 ; \ddot{p}} A_{\mathbf{i}_{1} \geqslant l_{0}, \mathbf{a} \geqslant l_{0}, \mathbf{t} \geqslant l_{0}}}^{t_{l_{0}+1}}(v, 1)$ of $Q_{\mathbf{i}, \mathbf{a} ; \ddot{p} A}^{\mathbf{t} A}(v, 1)$, and hence itself, is zero (see [BLW14, Lemma A.20]). Now the proposition follows by applying (9.2.8).

Now we discuss the stabilization of $\mathbf{S}_{n, d}^{\mathfrak{c}}$. We set ${ }_{p} A=A+p I_{n}$. The following proposition describes the relationship between the standard basis elements $[A]_{d}$ and the elements $\mathbf{f}_{A ; d}$ under the stabilization with respect to $p I_{n}$. Note that the partial orders $\leqslant$ alg and $\leqslant$ on $\Xi_{n, d}$ can be defined on $\tilde{\Xi}_{n, d}$ as well in exactly the same way.

Proposition 9.2.4. Let $A \in \widetilde{\Xi}_{n, d}$. There exist $Z_{i} \in \widetilde{\Xi}_{n, d}$, for $1 \leqslant i \leqslant m$, with $Z_{i}<A$, $Q_{i}\left(v, v^{\prime}\right) \in \mathscr{R}$ and $p_{0} \in \mathbb{N}$ such that

$$
\left[{ }_{p} A\right]_{d+\frac{p}{2} n}=\mathbf{f}_{p A ; d+\frac{p}{2} n}+\sum_{i=1}^{m} Q_{i}\left(v, v^{-p}\right)\left[{ }_{p} Z_{i}\right]_{d+\frac{p}{2} n}, \quad \forall p \geqslant p_{0}, p \in 2 \mathbb{N} .
$$

Proof. We transport the statement via $\rho$ to a similar one for $\ddot{\mathbf{f}}_{p A ; d+\frac{p}{2} n}$ in $\mathbf{S}_{\breve{n}, d+\frac{p}{2} n}^{\mathfrak{c}}$. The existence of $p_{0}, Z_{i}$ and $Q_{i}\left(v, v^{\prime}\right)$ follows by Proposition 9.2.3. The claim on the leading term follows from Theorem 9.1.6.

Now we can formulate the stabilization of the multiplication of $\mathbf{S}_{n, d}^{\mathfrak{c}}$.

Proposition 9.2.5. Assume that $A_{1}, \ldots, A_{l} \in \widetilde{\Xi}_{n, d}$ satisfy $\operatorname{co}\left(A_{i}\right)=\operatorname{ro}\left(A_{i+1}\right)$ for all $1 \leqslant i \leqslant$ $l-1$. There exist $Z_{1}, \ldots, Z_{m} \in \widetilde{\Xi}_{n, d}, G_{1}\left(v, v^{\prime}\right), \ldots, G_{m}\left(v, v^{\prime}\right) \in \mathscr{R}$, and $p_{0} \in \mathbb{N}$ such that

$$
\left.\left[{ }_{p} A_{1}\right]_{d+\frac{p}{2} n} *{ }_{p} A_{2}\right]_{d+\frac{p}{2} n} * \cdots *\left[{ }_{p} A_{l}\right]_{d+\frac{p}{2} n}=\sum_{i=1}^{m} G_{i}\left(v, v^{-p}\right)\left[{ }_{p} Z_{i}\right]_{d+\frac{p}{2} n}, \quad \forall p \geqslant p_{0}, p \in 2 \mathbb{N}
$$

Proof. By Proposition 9.2.3, we have a formula similar to (9.2.10) with ${ }_{p} A_{i}$ replaced by $\mathbf{f}_{p A_{i} ; d+\frac{p}{2} n}$. The proposition now follows by using Proposition 9.2.4 and an induction with respect to the partial order $\leqslant$ on the $A_{i}$ 's.

We have the following corollary to Theorem 9.1.6 and Proposition 9.2.5,

Corollary 9.2.6. For any matrix $A \in \widetilde{\Xi}_{n, d}$ of depth $m$, there exist tridiagonal matrices $A_{1}, A_{2}, \ldots, A_{m}$ in $\widetilde{\Xi}_{n, d}$ satisfying $\operatorname{ro}\left(A_{m}\right)=\operatorname{ro}(A), \operatorname{co}\left(A_{1}\right)=\operatorname{co}(A), \operatorname{ro}\left(A_{i}\right)=\operatorname{co}\left(A_{i+1}\right)$ for 
$1 \leqslant i \leqslant m-1$ and $A_{i}-\sum_{1 \leqslant j \leqslant n}\left(\sum_{k \leqslant j-i+1} a_{k, j+1}\right) E_{\theta}^{j, j+1}$ is diagonal for all $1 \leqslant i \leqslant m$ such that

$\left[{ }_{p} A_{m}\right]_{d+\frac{p}{2} n} *\left[{ }_{p} A_{m-1}\right]_{d+\frac{p}{2} n} * \cdots *\left[{ }_{p} A_{1}\right]_{d+\frac{p}{2} n}=\left[{ }_{p} A\right]_{d+\frac{p}{2} n}+\sum_{i=1}^{l} G_{i}\left(v, v^{-p}\right)\left[{ }_{p} Z_{i}\right]_{d+\frac{p}{2} n}, \forall p \in 2 \mathbb{N}, p \geqslant p_{0}$, where $p_{0}, G_{i}\left(v, v^{\prime}\right) \in \mathscr{R}$ and $Z_{1}, \ldots, Z_{l} \in \widetilde{\Xi}_{n, d}$ are as in Proposition 9.2.5 such that $Z_{i}<A$.

The following stabilization of the bar operator on $\mathbf{S}_{n, d}^{\mathfrak{c}}$ is a counterpart of [BLM90, Proposition 4.3]. It can be proved in the same way by induction with respect to the partial order $\leqslant$ on $A$, with the help of (9.2.9) and Corollary 9.2.6, we skip the detail.

Proposition 9.2.7. Assume that $A \in \widetilde{\Xi}_{n, d}$. Then there exist $Y_{i} \in \widetilde{\Xi}_{n, d}$ with $Y_{i}<A$, $H_{i}\left(v, v^{\prime}\right) \in \mathscr{R}$ for all $1 \leqslant i \leqslant s$ and $p_{0} \in \mathbb{N}$ such that

$$
{\overline{\left[{ }_{p} A\right.}}_{d+\frac{p}{2} n}=\left[{ }_{p} A\right]_{d+\frac{p}{2} n}+\sum_{i=1}^{s} H_{i}\left(v, v^{-p}\right)\left[{ }_{p} Y_{i}\right]_{d+\frac{p}{2} n}, \quad \forall p \geqslant p_{0}, p \in 2 \mathbb{N} .
$$

\subsection{Comultiplication And stabilization}

In the section, we take advantage of the embedding $\rho: \mathbf{S}_{n, d}^{\mathfrak{c}} \rightarrow \mathbf{U}_{\breve{n}, d}^{\mathfrak{c}}$ to study the coassociativity and stability behavior of the comultiplication $\Delta^{\mathfrak{c}}: \mathbf{S}_{n, d}^{\mathfrak{c}} \longrightarrow \mathbf{S}_{n, d^{\prime}}^{\mathfrak{c}} \otimes \mathbf{S}_{n, d^{\prime \prime}}\left(\right.$ recall $\Delta^{\mathfrak{c}}$ was defined in (5.3.6) ).

To avoid any ambiguity, we put a subscript $n$ to the comultiplication $\Delta^{\mathfrak{c}}$ of $\mathbf{S}_{n, d}^{\mathfrak{c}}$, and use $\Delta_{\breve{n}}^{\mathfrak{c}}$ for that on $\mathbf{S}_{\breve{n}, d}^{\mathfrak{c}}$. We apply the same convention to the imbedding $\rho$ too. Note that exactly the same definition gives rise to an imbedding $\mathbf{S}_{n, d} \rightarrow \mathbf{S}_{\breve{n}, d}$, which we shall again denote by $\rho_{d}$. The following lemma shows the compatibility of the comultiplications and the imbedding $\rho$.

Lemma 9.3.1. The following diagram is commutative:

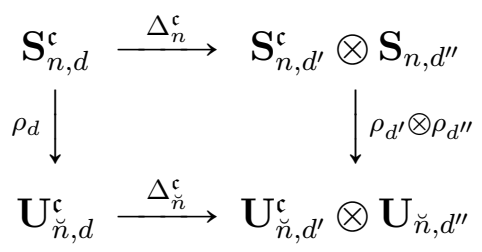

Proof. By definitions, we have a similar commutative diagram with the $\Delta^{\mathfrak{c}}$ 's replaced by the raw ones $\widetilde{\Delta}^{\mathfrak{c}}$; cf. (5.2.1). Now the twists $s\left(\mathbf{b}^{\prime}, \mathbf{a}^{\prime}, \mathbf{b}^{\prime \prime}, \mathbf{a}^{\prime \prime}\right)$ and $u\left(\mathbf{b}^{\prime \prime}, \mathbf{a}^{\prime \prime}\right)$ remain unchanged under the obvious imbeddings $\Lambda_{n, d} \rightarrow \Lambda_{\breve{n}, d}$ and $\Lambda_{n, d}^{\mathfrak{c}} \rightarrow \Lambda_{\breve{n}, d}^{\mathfrak{c}}$. This immediately shows that the commutative diagram for $\widetilde{\Delta}^{\mathfrak{c}}$ s can be extended to the one in the lemma.

Proposition 9.3.2. The comultiplication $\Delta^{\mathfrak{c}}$ on $\mathbf{S}_{n, d}^{\mathfrak{c}}$ is coassociative, that is,

$$
(1 \otimes \Delta) \Delta^{\mathfrak{c}}=\left(\Delta^{\mathfrak{c}} \otimes 1\right) \Delta^{\mathfrak{c}}
$$

Proof. By Lemma 9.3.1, this is a consequence of the fact that the restriction of $\Delta_{\breve{n}}^{\mathfrak{c}}$ to $\mathbf{U}_{\breve{n}, d}^{c}$ is coassociative in Proposition 5.3.5.

Remark 9.3.3. Recall the comultiplication $\Delta$ on $\mathbf{S}_{n, d}$ of affine type $A$ from (5.3.8). It follows by the same argument as above that the comultiplication $\Delta$ on $\mathbf{S}_{n, d}$ is coassociative, that is, $(1 \otimes \Delta) \Delta=(\Delta \otimes 1) \Delta$. 
Now, we study the stabilization behavior of the comultiplication $\Delta_{n}^{\mathfrak{c}}$ (cf. (5.3.6)) as $d$ varies. Recall the notation $\models$ from Section 6.5. We generalize it as follows. For any $\lambda^{\prime}, \lambda$ in $\mathbb{Z}_{n}^{\mathfrak{c}}$ and $\lambda^{\prime \prime} \in \mathbb{Z}_{n}$, we say that $\left(\lambda^{\prime}, \lambda^{\prime \prime}\right) \models \lambda$ if $\lambda_{i}=\lambda_{i}^{\prime}+\lambda_{i}^{\prime \prime}+\lambda_{-i}^{\prime \prime}$ for all $i$. Let

$$
\Delta_{\mathbf{b}^{\prime}, \mathbf{a}^{\prime}, \mathbf{b}^{\prime \prime}, \mathbf{a}^{\prime \prime}}^{\mathfrak{c}}: \mathbf{S}_{n, d}^{\mathfrak{c}}(\mathbf{b}, \mathbf{a}) \longrightarrow \mathbf{S}_{n, d}^{\mathfrak{c}} \stackrel{\Delta^{\mathfrak{c}}}{\longrightarrow} \mathbf{S}_{n, d^{\prime}}^{\mathfrak{c}} \otimes \mathbf{S}_{n, d^{\prime \prime}} \longrightarrow \mathbf{S}_{n, d}^{\mathfrak{c}}\left(\mathbf{b}^{\prime}, \mathbf{a}^{\prime}\right) \otimes \mathbf{S}_{n, d^{\prime \prime}}\left(\mathbf{b}^{\prime \prime}, \mathbf{a}^{\prime \prime}\right)
$$

be a component of $\Delta^{\mathfrak{c}}$ with $\left(\mathbf{b}^{\prime}, \mathbf{b}^{\prime \prime}\right) \models \mathbf{b},\left(\mathbf{a}^{\prime}, \mathbf{a}^{\prime \prime}\right) \models \mathbf{a}$, where the first and third maps are the natural inclusion and projection, respectively. Recall the notation ${ }_{p} \mathbf{a}=\mathbf{a}+(\ldots, p, p, p, \ldots)$. We put

$$
{ }_{p} \Delta_{\mathbf{b}^{\prime}, \mathbf{a}^{\prime}, \mathbf{b}^{\prime \prime}, \mathbf{a}^{\prime \prime}}^{\mathfrak{c}}=\Delta_{p}^{\mathfrak{c}} \mathbf{b}^{\prime},{ }_{p} \mathbf{a}^{\prime}, \mathbf{b}^{\prime \prime},{ }_{p} \mathbf{a}^{\prime \prime}
$$

Proposition 9.3.4. Assume that $d^{\prime}+d^{\prime \prime}=d$ and let $\mathbf{b}^{\prime}, \mathbf{a}^{\prime} \in \mathbb{Z}_{n}^{\mathfrak{c}}$ and $\mathbf{b}^{\prime \prime}, \mathbf{a}^{\prime \prime} \in \mathbb{Z}_{n}$ be so that ${ }_{p} \Delta_{\mathbf{b}^{\prime}, \mathbf{a}^{\prime}, \mathbf{b}^{\prime \prime}, \mathbf{a}^{\prime \prime}}$ is defined. Fix $A \in \widetilde{\Xi}_{n, d}$. There exist matrices $A_{i}^{\prime} \in \widetilde{\Xi}_{n, d^{\prime}}$ where $1 \leqslant i \leqslant l$ for some $l$, matrices $A_{j}^{\prime \prime}$ in $\widetilde{\Theta}_{n, d^{\prime \prime}}$ where $1 \leqslant j \leqslant m$ for some $m, C_{i, j}\left(v, v^{\prime}\right) \in \mathscr{R}$ for $1 \leqslant i \leqslant l$, $1 \leqslant j \leqslant m$, and $p_{0} \in \mathbb{N}$ such that

$$
{ }_{p} \Delta_{\mathbf{b}^{\prime}, \mathbf{a}^{\prime}, \mathbf{b}^{\prime \prime}, \mathbf{a}^{\prime \prime}}\left(\left[{ }_{2 p} A\right]_{d+p n}\right)=\sum_{1 \leqslant i \leqslant l, 1 \leqslant j \leqslant m} C_{i, j}\left(v, v^{-p}\right)\left[{ }_{p} A_{i}^{\prime}\right]_{d^{\prime}+\frac{p}{2} n} \otimes{ }^{\mathfrak{a}}\left[{ }_{p} A_{j}^{\prime \prime}\right]_{d^{\prime \prime}+p n}, \quad \forall p \geqslant p_{0}, p \in 2 \mathbb{N} .
$$

Proof. We prove this by induction with respect to the partial order on $A$. By Proposition 9.2.4, we have

$$
\left[{ }_{2 p} A\right]_{d+p n}=\mathbf{f}_{2 p} A ; d+p n+\sum_{i=1}^{m} Q_{i}\left(v, v^{-2 p}\right)\left[{ }_{2 p} Z_{i}\right]_{d+p n}, \quad \forall p \geqslant p_{0}, p \in 2 \mathbb{N} .
$$

If we define $\tilde{Q}_{i}\left(v, v^{\prime}\right)=Q_{i}\left(v, v^{\prime 2}\right)$ for all $i$, then we can rewrite the above equality as

$$
\left[{ }_{2 p} A\right]_{d+p n}=\mathbf{f}_{2 p} A ; d+p n+\sum_{i=1}^{m} \tilde{Q}_{i}\left(v, v^{-p}\right)\left[{ }_{2 p} Z_{i}\right]_{d+p n}, \quad \forall p \geqslant p_{0}, p \in 2 \mathbb{N} .
$$

With this equality and by induction, it is reduced to proving a similar statement with $[A]_{d}$ replaced by $\mathbf{f}_{A ; d}$. By Lemma 9.3.1, this is in turn reduced to proving a similar result for $\ddot{\mathbf{f}}_{A ; d}$, which is then a consequence of Proposition 9.2.3 and Proposition [5.3.4. The proposition follows.

\subsection{The ALGEBRA $\dot{\mathbf{K}}_{n}^{\mathfrak{c}}$ AND ITS STABly CANONICAL BASIS}

Let $\dot{\mathbf{K}}_{n}^{\mathfrak{c}}$ be the vector space over $\mathbb{Q}(v)$ spanned by the formal symbols $[A]$ where $A \in \widetilde{\Xi}_{n}$. By Proposition 9.2.5 and applying a standard argument, the space $\dot{\mathbf{K}}_{n}^{\mathfrak{c}}$ becomes an associative algebra without unit with the product

$$
\left[A_{1}\right] \cdot\left[A_{2}\right]=\sum_{i=1}^{m} G_{i}(v, 1)\left[Z_{i}\right], \quad \forall A_{1}, A_{2} \in \widetilde{\Xi}_{n},
$$

where $G_{i}\left(v, v^{\prime}\right)$ and $Z_{i}$ for all $1 \leqslant i \leqslant m$ are as in Proposition 9.2.5. Corollary 9.2.6 implies the following.

Proposition 9.4.1. For any matrix $A$ in $\widetilde{\Xi}_{n}$ of depth $m$, there exist tridiagonal matrices $A_{1}, A_{2}, \ldots, A_{m} \in \widetilde{\Xi}_{n}$ satisfying $\operatorname{ro}\left(A_{m}\right)=\operatorname{ro}(A), \operatorname{co}\left(A_{1}\right)=\operatorname{co}(A), \operatorname{ro}\left(A_{i}\right)=\operatorname{co}\left(A_{i+1}\right)$ for 
$1 \leqslant i \leqslant m-1$ and $A_{i}-\sum_{1 \leqslant j \leqslant n}\left(\sum_{k \leqslant j-i+1} a_{k, j+1}\right) E_{\theta}^{j, j+1}$ is diagonal for all $1 \leqslant i \leqslant m$ such that

$$
m_{A}^{\prime}:=\left[A_{m}\right] \cdot\left[A_{m-1}\right] \cdot \ldots \cdot\left[A_{1}\right]=[A]+\text { lower terms. }
$$

Thus $\left\{m_{A}^{\prime} \mid A \in \widetilde{\Xi}_{n}\right\}$ forms a basis for $\dot{\mathbf{K}}_{n}^{\mathfrak{c}}$ (called a semi-monomial basis). Notice that the element $m_{A}^{\prime}$ is not necessarily bar-invariant.

For each matrix $A \in \widetilde{\Xi}_{n}$, we define the element $\mathbf{f}_{A} \in \dot{\mathbf{K}}_{n}^{\mathfrak{c}}$ to be

$$
\mathbf{f}_{A}=[A]+\sum_{i=1}^{m} Q_{i}(v, 1)\left[Z_{i}\right]
$$

where $Q_{i}\left(v, v^{\prime}\right)$ and $Z_{i}$ are in (9.2.9). In particular, we have

$$
\mathbf{f}_{A}=[A]+\text { lower terms. }
$$

Moreover, we can give a more precise description of $Q_{i}\left(v, v^{\prime}\right)$ and $Z_{i}$. By the definition of $\mathbf{f}_{A}$ in (9.1.8) (also see (9.1.5)) and Proposition 9.2.3, we have the following.

Proposition 9.4.2. For any matrix $A \in \widetilde{\Xi}_{n, d}$, there exists a pair of tuples (i,a) such that

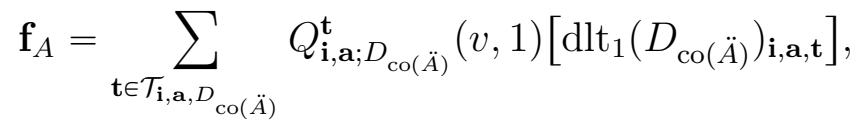

where $D_{\operatorname{co}(\ddot{A})}$ is the diagonal matrix in $\widetilde{\Xi}_{\breve{n}, d}$ with diagonal $\operatorname{co}(\ddot{A})$ and $\operatorname{dlt}_{1}$ is the deleting operation inverse to the operation .".

Assume that $B \in \widetilde{\Xi}_{n, d}$ and $B-\sum_{1 \leqslant i \leqslant n} \beta_{i} E_{\theta}^{i, i+1}$ is diagonal. Let $\mathbf{i}_{0}$ and $\mathbf{b}_{0}$ denote the sequences of subscripts and superscripts in the left hand side of (9.1.4) (with $A$ replaced by $B)$, respectively, that is,

$$
\mathbf{i}_{0}=(0, n, n+1, n-1, n, n-2, \cdots, 1), \quad \mathbf{b}_{0}=\left(\beta_{0}, \beta_{n-1}, \beta_{n-1}, \beta_{n-2}, \cdots, \beta_{0}\right),
$$

where $\beta_{0}=\beta_{n}$ The following multiplication formula in $\dot{\mathbf{K}}_{n}^{\mathfrak{c}}$ follows by Proposition 9.2 .3 .

Proposition 9.4.3. Let $A, B \in \widetilde{\Xi}_{n}$ be such that $\operatorname{co}(B)=\operatorname{ro}(A)$ and $B-\sum_{1 \leqslant i \leqslant n} \beta_{i} E_{\theta}^{i, i+1}$ is diagonal. Then the following multiplication formula holds in $\dot{\mathbf{K}}_{n}^{\mathfrak{c}}$ :

$$
\mathbf{f}_{B} \cdot[A]=\sum_{\mathbf{t} \in \mathcal{T}_{\mathbf{i}_{0}, \mathbf{b}_{0}, \ddot{A}}} Q_{\mathbf{i}_{0}, \mathbf{b}_{0} ; \ddot{A}}^{\mathbf{t}}(v, 1)\left[\operatorname{dlt}_{1}(\ddot{A})_{\mathbf{i}_{0}, \mathbf{b}_{0}, \mathbf{t}}\right] .
$$

Now we show that the element $\mathbf{f}_{A} \in \dot{\mathbf{K}}_{n}^{\mathfrak{c}}$ can be expressed as a monomial in $\mathbf{f}_{A_{i}}$ for various tridiagonal matrices $A_{i}$ (similar to the Schur algebra case).

Proposition 9.4.4. Let $A \in \widetilde{\Xi}_{n}$, and we retain the notations of tridiagonal matrices $A_{i}$ from Proposition 9.4.1. Then we have

$$
\mathbf{f}_{A}=\mathbf{f}_{A_{m}} \cdot \mathbf{f}_{A_{m-1}} \cdot \ldots \cdot \mathbf{f}_{A_{1}} .
$$

Moreover, we have $\overline{\mathbf{f}_{A}}=\mathbf{f}_{A}$. 
Proof. Let $\dot{\mathbf{K}}_{n, \mathscr{R}}^{\mathfrak{c}}$ be the free $\mathscr{R}$-module spanned by the matrices in $\widetilde{\Xi}_{n}$. Similar to (9.4.1), we can define an associative algebra over $\mathscr{R}$ by

$$
A_{1} \cdot{ }^{\prime} A_{2}=\sum_{i=1}^{m} G_{i}\left(v, v^{\prime}\right) Z_{i}, \quad \forall A_{1}, A_{2} \in \widetilde{\Xi}_{n} .
$$

Similar to (9.4.2), we can define

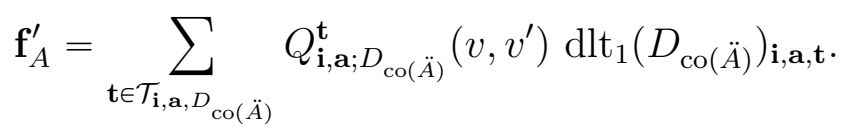

Then by Proposition 9.2.3, we have

$$
\mathbf{f}_{A}^{\prime}=\mathbf{f}_{A_{m}}^{\prime} \cdot \mathbf{f}_{A_{m-1}}^{\prime} \cdot \ldots \cdot \cdot^{\prime} \mathbf{f}_{A_{1}}^{\prime} .
$$

By specializing $v^{\prime}$ at $v^{\prime}=1$, we obtain the product formula for $\mathbf{f}_{A}$.

The bar invariance of $\mathbf{f}_{A}$ follows from the same fact on the Schur algebra level and the formal stabilization procedure as above. We skip the detail.

By Proposition 9.2.7. we can define a bar involution on $\dot{\mathbf{K}}_{n}^{\mathfrak{c}}$ by $\bar{v}=v^{-1}$ and letting

$$
\overline{[A]}=[A]+\sum_{i=1}^{s} H_{i}(v, 1)\left[Y_{i}\right], \quad \forall A \in \widetilde{\Xi}_{n},
$$

where $H_{i}\left(v, v^{\prime}\right)$ and $Y_{i}<A$ are as in Proposition 9.2.7. The next proposition follows by a standard argument.

Proposition 9.4.5. For any $A \in \widetilde{\Xi}_{n}$, there exists a unique element $\{A\}$ in $\dot{\mathbf{K}}_{n}^{\mathfrak{c}}$ such that

$$
\overline{\{A\}}=\{A\}, \quad\{A\}=[A]+\sum_{A^{\prime}<A} \pi_{A, A^{\prime}}\left[A^{\prime}\right], \quad \pi_{A, A^{\prime}} \in v^{-1} \mathbb{Z}\left[v^{-1}\right] .
$$

Moreover, $\left\{\{A\} \mid A \in \widetilde{\Xi}_{n}\right\}$ forms a basis for $\dot{\mathbf{K}}_{n}^{\mathfrak{c}}$ (called the stably canonical basis).

Let us summarize the main results of this section.

Theorem 9.4.6. The algebra $\dot{\mathbf{K}}_{n}^{\mathfrak{c}}$ admits a standard basis $\left\{[A] \mid A \in \widetilde{\Xi}_{n}\right\}$, a semi-monomial basis $\left\{m_{A}^{\prime} \mid A \in \widetilde{\Xi}_{n}\right\}$, a monomial basis $\left\{\mathbf{f}_{A} \mid A \in \widetilde{\Xi}_{n}\right\}$, and a stably canonical basis $\left\{\{A\} \mid A \in \widetilde{\Xi}_{n}\right\}$.

\subsection{The Algebra $\dot{\mathbf{K}}_{n}$ OF Affine type $A$ And its COMultiplication}

In this section, we revisit the Schur algebras of affine type $A$ and study its stabilization algebra $\dot{\mathbf{K}}_{n}$. The constructions in this section will serve as a prerequisites for the constructions of the comultiplication of the algebra $\dot{\mathbf{K}}_{n}^{\mathfrak{c}}$ in the following section.

Recall the comultiplication $\Delta$ from (5.3.8) of affine type $A$. The following stabilization for the comultiplication $\Delta$ at the Schur algebra level is the counterpart of Proposition 9.3.4 which can be proved in the same way.

Proposition 9.5.1. Assume that $d^{\prime}+d^{\prime \prime}=d$, and let $\mathbf{b}^{\prime}, \mathbf{a}^{\prime}, \mathbf{b}^{\prime \prime}, \mathbf{a}^{\prime \prime} \in \mathbb{Z}_{n}$ be so that ${ }_{p} \Delta_{\mathbf{b}^{\prime}, \mathbf{a}^{\prime}, \mathbf{b}^{\prime \prime}, \mathbf{a}^{\prime \prime}}$ is defined. For each $A \in \widetilde{\Theta}_{n, d}$, there exist $A_{i}^{\prime} \in \widetilde{\Theta}_{n, d^{\prime}}$ where $1 \leqslant i \leqslant l$ for some $l, A_{j}^{\prime \prime} \in \widetilde{\Theta}_{n, d^{\prime \prime}}$ where $1 \leqslant j \leqslant m$ for some $m,{ }^{\mathfrak{a}} C_{i, j}\left(v, v^{\prime}\right) \in \mathscr{R}$ for $1 \leqslant i \leqslant l, 1 \leqslant j \leqslant m$, and $p_{0} \in \mathbb{N}$ such that

$$
{ }_{p} \Delta_{\mathbf{b}^{\prime}, \mathbf{a}^{\prime}, \mathbf{b}^{\prime \prime}, \mathbf{a}^{\prime \prime}}\left({ }^{\mathfrak{a}}\left[{ }_{2 p} A\right]_{d+2 p n}\right)=\sum_{1 \leqslant i \leqslant l, 1 \leqslant j \leqslant m}{ }^{\mathfrak{a}} C_{i, j}\left(v, v^{-p}\right){ }^{\mathfrak{a}}\left[{ }_{p} A_{i}^{\prime}\right]_{d^{\prime}+p n} \otimes{ }^{\mathfrak{a}}\left[{ }_{p} A_{j}^{\prime \prime}\right]_{d^{\prime \prime}+p n}, \quad \forall p \geqslant p_{0} .
$$


Let

$$
\widetilde{\Theta}_{n}=\left\{A=\left(a_{i j}\right)_{i, j \in \mathbb{Z}} \mid a_{i j} \in \mathbb{N}, \forall i \neq j, a_{i i} \in \mathbb{Z}, \forall i \in \mathbb{Z}\right\} .
$$

Let $\dot{\mathbf{K}}_{n}$ be the vector space over $\mathbb{Q}(v)$ spanned by the symbols ${ }^{\mathfrak{a}}[A]$ for all $A \in \widetilde{\Theta}_{n}$. Replacing $\mathbf{S}_{n, d}^{\mathfrak{c}}$ by the Schur algebra $\mathbf{S}_{n, d}$ from Chapter 2 and repeating the constructions in the preceding sections, we can endow $\dot{\mathbf{K}}_{n}$ with an associative algebra structure, a bar involution, a canonical basis $\left\{\mathfrak{a}\{A\} \mid A \in \widetilde{\Theta}_{n}\right\}$. Indeed the treatment is much simpler in the current type $A$ setting since the analogous basis elements ${ }^{\mathfrak{a}} \mathbf{f}_{A}$ and ${ }^{\mathfrak{a}}[A]$ coincides when $A$ is tridiagonal.

Remark 9.5.2. The associative algebra structure on $\dot{\mathbf{K}}_{n}$ and its stably canonical basis were first introduced in [DF13] by a completely different and Hecke algebraic approach, (also see [LL15]). Moreover, they showed that $\dot{\mathbf{K}}_{n}$ is isomorphic to the idempotented quantum affine $\mathfrak{g l}_{n}, \dot{\mathbf{U}}\left(\widehat{\mathfrak{g l}}_{n}\right)$.

Moreover, by Proposition 9.5.1, we can define a comultiplication for $\dot{\mathbf{K}}_{n}$ as follows. Let ${ }_{\mathbf{b}} \dot{\mathbf{K}}_{\mathbf{a}}$, for any $\mathbf{b}, \mathbf{a} \in \mathbb{Z}_{n}$, be the subspace of $\dot{\mathbf{K}}_{n}$ spanned by the standard basis elements ${ }^{\mathfrak{a}}[A]$ such that $\operatorname{ro}(A)=\mathbf{b}$ and $\operatorname{co}(A)=\mathbf{a}$. For any $\mathbf{b}, \mathbf{a}, \mathbf{b}^{\prime}, \mathbf{a}^{\prime}, \mathbf{b}^{\prime \prime}, \mathbf{a}^{\prime \prime} \in \mathbb{Z}_{n}$ such that $\mathbf{b}^{\prime}+\mathbf{b}^{\prime \prime}=\mathbf{b}$ and $\mathbf{a}^{\prime}+\mathbf{a}^{\prime \prime}=\mathbf{a}$, we define a linear map

$$
\begin{aligned}
\Delta_{\mathbf{b}^{\prime}, \mathbf{a}^{\prime}, \mathbf{b}^{\prime \prime}, \mathbf{a}^{\prime \prime}} & :{ }_{\mathbf{b}} \dot{\mathbf{K}}_{\mathbf{a}} \longrightarrow{ }_{\mathbf{b}^{\prime}} \dot{\mathbf{K}}_{\mathbf{a}^{\prime}} \otimes{ }_{\mathbf{b}^{\prime \prime}} \dot{\mathbf{K}}_{\mathbf{a}^{\prime \prime}}, \\
\Delta_{\mathbf{b}^{\prime}, \mathbf{a}^{\prime}, \mathbf{b}^{\prime \prime}, \mathbf{a}^{\prime \prime}}\left({ }^{\mathfrak{a}}[A]\right) & =\sum_{1 \leqslant i \leqslant l, 1 \leqslant j \leqslant m}{ }^{\mathfrak{a}} C_{i, j}(v, 1){ }^{\mathfrak{a}}\left[A_{i}^{\prime}\right] \otimes{ }^{\mathfrak{a}}\left[A_{j}^{\prime \prime}\right],
\end{aligned}
$$

where $A_{i}^{\prime}, A_{j}^{\prime \prime},{ }^{\mathfrak{a}} C_{i j}\left(v, v^{\prime}\right)$ are from Proposition 9.5.1. We shall call the collection

$$
\dot{\Delta}=\left(\Delta_{\mathbf{b}^{\prime}, \mathbf{a}^{\prime}, \mathbf{b}^{\prime \prime}, \mathbf{a}^{\prime \prime}}\right)_{\mathbf{b}^{\prime}, \mathbf{a}^{\prime}, \mathbf{b}^{\prime \prime}, \mathbf{a}^{\prime \prime} \in \mathbb{Z}_{n}}
$$

the comultiplication of $\dot{\mathbf{K}}_{n}$. Let ${ }^{\mathfrak{a}} g_{A, B}^{C}$ and ${ }^{\mathfrak{a}} h_{A}^{B, C}$ denote the structure constants with respect to the multiplication and comultiplication in $\dot{\mathbf{K}}_{n}$, respectively, i.e.,

$$
\begin{aligned}
{ }^{\mathfrak{a}}[A] \cdot{ }^{\mathfrak{a}}[B] & =\sum_{C \in \widetilde{\Theta}_{n}}{ }^{\mathfrak{a}} g_{A, B}^{C}{ }^{\mathfrak{a}}[C], \\
\Delta_{\mathbf{b}^{\prime}, \mathbf{a}^{\prime}, \mathbf{b}^{\prime \prime}, \mathbf{a}^{\prime \prime}}\left({ }^{\mathfrak{a}}[A]\right) & =\sum_{B, C \in \widetilde{\Theta}_{n}}{ }^{\mathfrak{a}} h_{A}^{B, C}{ }^{\mathfrak{a}}[B] \otimes{ }^{\mathfrak{a}}[C] .
\end{aligned}
$$

Proposition 9.5.3. The comultiplication $\dot{\Delta}$ is an algebra homomorphism in the following sense: for all matrices $A, B, C^{\prime}, C^{\prime \prime} \in \widetilde{\Theta}_{n}$, one has

$$
\sum_{C \in \widetilde{\Theta}_{n}}{ }^{\mathfrak{a}} g_{A, B}^{C}{ }^{\mathfrak{a}} h_{C}^{C^{\prime}, C^{\prime \prime}}=\sum_{A^{\prime}, A^{\prime \prime}, B^{\prime}, B^{\prime \prime} \in \widetilde{\Theta}_{n}}{ }^{\mathfrak{a}} h_{A}^{A^{\prime}, A^{\prime \prime} \mathfrak{a}} h_{B}^{B^{\prime}, B^{\prime \prime} \mathfrak{a}} g_{A^{\prime}, B^{\prime}}^{C^{\prime}}{ }^{\mathfrak{a}} g_{A^{\prime \prime}, B^{\prime \prime}}^{C^{\prime \prime}}
$$

Proof. We first show that the sums in the two sides of the equation (9.5.1) are finite. For two fixed matrices $A, B$ in $\widetilde{\Theta}_{n}$, there are only finitely many $C \in \widetilde{\Theta}_{n}$ such that $g_{A, B}^{C} \neq 0$ by definition. So the sum on the left-hand side is finite. To see that the sum on the righthand side of (9.5.1) is finite, we first observe that for each $A \in \widetilde{\Theta}_{n}$, if the structure constant ${ }^{\mathfrak{a}} h_{A}^{A^{\prime}, A^{\prime \prime}}$ is nonzero, then $A^{\prime}, A^{\prime \prime} \leqslant$ alg $A$. Next we observe that for $\mathbf{c}^{\prime}, \mathbf{c}^{\prime \prime} \in \mathbb{Z}_{n}$, the set $\left\{\left(A^{\prime}, A^{\prime \prime}\right) \mid{ }^{\mathfrak{a}} h_{A}^{A^{\prime}, A^{\prime \prime}} \neq 0, \operatorname{ro}\left(A^{\prime}\right)=\mathbf{c}^{\prime}, \operatorname{co}\left(A^{\prime \prime}\right)=\mathbf{c}^{\prime \prime}\right\}$ is finite. This is because if $A^{\prime}, A^{\prime \prime} \leqslant$ alg $A$, then the number of the choices for the entry $(i, j)$ for $i \neq j$ of $A^{\prime}$ and $A^{\prime \prime}$ is finite. Now the row and column vectors of $A^{\prime}$ and $A^{\prime \prime}$ are fixed respectively, forcing the choice of the diagonal 
entries of $A^{\prime}$ and $A^{\prime \prime}$ to be finite. In the sum of the right-hand side of (9.5.1), we must have that $\operatorname{ro}\left(A^{\prime}\right)=\operatorname{ro}\left(C^{\prime}\right), \operatorname{co}\left(B^{\prime}\right)=\operatorname{co}\left(C^{\prime}\right), \operatorname{ro}\left(A^{\prime \prime}\right)=\operatorname{ro}\left(C^{\prime \prime}\right)$ and $\operatorname{co}\left(B^{\prime \prime}\right)=\operatorname{co}\left(C^{\prime \prime}\right)$, which are fixed. So the sum on the righthand side of (9.5.1) is indeed finite.

Once we observe that both sums in (9.5.1) are finite, the proof of the equation is reduced to showing a similar equation on the level of the Schur algebra $\mathbf{S}_{n, d}$ for very large $d$, which is in turn equivalent to the fact that the comultiplication $\Delta$ on $\mathbf{S}_{n, d}$ is an algebra homomorphism in [FL15]. The proposition is thus proved.

Proposition 9.5.3 can be equivalently reformulated as the following commutative diagram: for all tuples $\mathbf{a}, \mathbf{a}^{\prime}, \mathbf{a}^{\prime \prime}, \mathbf{b}, \mathbf{b}^{\prime}, \mathbf{b}^{\prime \prime}, \mathbf{c} \in \mathbb{Z}_{n}$ such that $\mathbf{b}^{\prime}+\mathbf{b}^{\prime \prime}=\mathbf{b}$ and $\mathbf{a}^{\prime}+\mathbf{a}^{\prime \prime}=\mathbf{a}$, we have

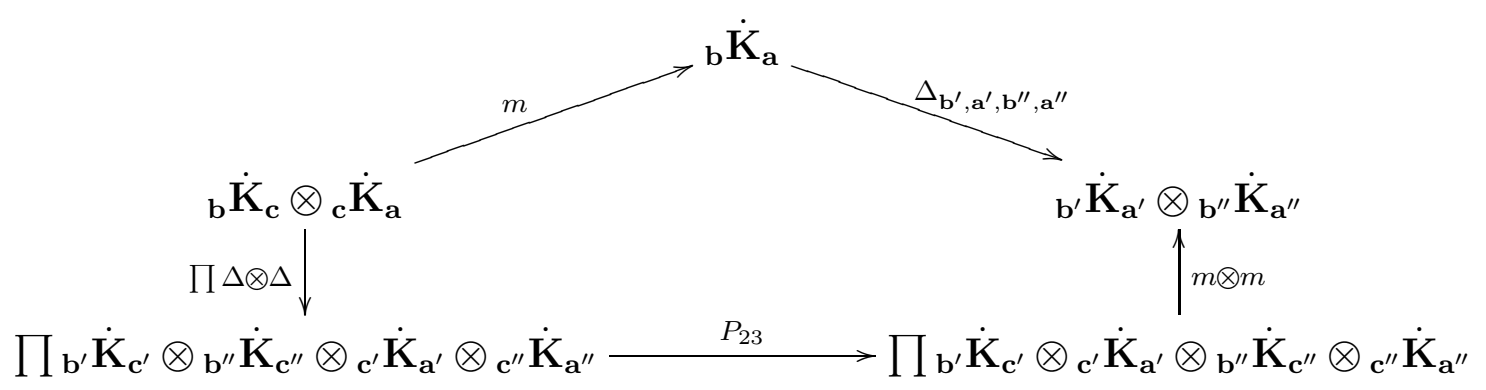

where $m$ represents the multiplication of $\dot{\mathbf{K}}_{n}$, all products run over all tuples $\left(\mathbf{c}^{\prime}, \mathbf{c}^{\prime \prime}\right)$ such that $\mathbf{c}^{\prime}+\mathbf{c}^{\prime \prime}=\mathbf{c}, \prod \Delta \otimes \Delta$ stands for the product of $\Delta_{\mathbf{b}^{\prime}, \mathbf{c}^{\prime}, \mathbf{b}^{\prime \prime}, \mathbf{c}^{\prime \prime}} \otimes \Delta_{\mathbf{c}^{\prime}, \mathbf{a}^{\prime}, \mathbf{c}^{\prime \prime}, \mathbf{a}^{\prime \prime}}$ and $P_{23}$ permutes the second and third entries.

Proposition 9.5.4. The comultiplication $\dot{\Delta}$ is coassociative in the following sense: for any matrices $A, A^{\prime}, A^{\prime \prime}, A^{\prime \prime \prime} \in \widetilde{\Theta}_{n}$, we have

$$
\sum_{B \in \widetilde{\Theta}_{n}}{ }^{\mathfrak{a}} h_{A}^{B, A^{\prime \prime \prime} \mathfrak{a}} h_{B}^{A^{\prime}, A^{\prime \prime}}=\sum_{B \in \widetilde{\Theta}_{n}}{ }^{\mathfrak{a}} h_{A}^{A^{\prime}, B} \mathfrak{a}^{\mathfrak{a}} h_{B}^{A^{\prime \prime}, A^{\prime \prime \prime}} .
$$

Proof. By arguing in a similar way as in the proof of Proposition 9.5.3, we see that both sums in (9.5.3) are finite. The equality can then be reduced to proving a similar equation on the Schur algebra level as in the proof of Proposition 9.5.3, which in turn follows by the coassociativity in Remark 9.3.3.

Proposition 9.5.4 can be equivalently reformulated as the following commutative diagram: for all sequences $\mathbf{a}, \mathbf{a}^{\prime}, \mathbf{a}^{\prime \prime}, \mathbf{a}^{\prime \prime \prime}, \mathbf{b}, \mathbf{b}^{\prime}, \mathbf{b}^{\prime \prime}, \mathbf{b}^{\prime \prime \prime} \in \mathbb{Z}_{n}$, we have

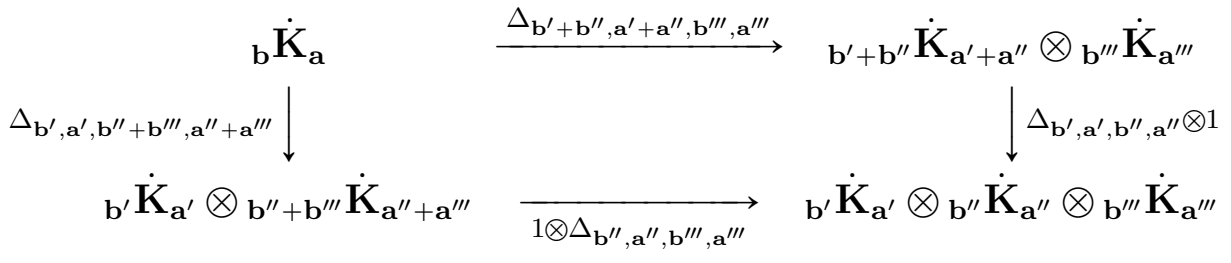

\subsection{The COMultiplication ON $\dot{\mathbf{K}}_{n}^{\mathfrak{c}}$}

Recall $\dot{\mathbf{K}}_{n}^{\mathfrak{c}}$ from Section 9.4 . For any $\mathbf{a}, \mathbf{b} \in \mathbb{Z}_{n}^{\mathfrak{c}}$, let ${ }_{\mathbf{b}} \dot{\mathbf{K}}_{\mathbf{a}}^{\mathfrak{c}}$ denote the subspace of $\dot{\mathbf{K}}_{n}^{\mathfrak{c}}$ spanned by the standard basis element $[A]$ such that $\operatorname{ro}(A)=\mathbf{b}$ and $\operatorname{co}(A)=\mathbf{a}$. For any 
$\mathbf{b}, \mathbf{a}, \mathbf{b}^{\prime}, \mathbf{a}^{\prime} \in \mathbb{Z}_{n}^{\mathfrak{c}}$ and $\mathbf{b}^{\prime \prime}, \mathbf{a}^{\prime \prime} \in \mathbb{Z}_{n}$ such that $\left(\mathbf{b}^{\prime}, \mathbf{b}^{\prime \prime}\right) \models \mathbf{b}$ and $\left(\mathbf{b}^{\prime \prime}, \mathbf{a}^{\prime \prime}\right) \models \mathbf{a}$, we define a linear map

$$
\begin{aligned}
& \Delta_{\mathbf{b}^{\prime}, \mathbf{a}^{\prime}, \mathbf{b}^{\prime \prime}, \mathbf{a}^{\prime \prime}}^{\mathfrak{c}}:{ }_{\mathbf{b}} \dot{\mathbf{K}}_{\mathbf{a}}^{\mathfrak{c}} \longrightarrow{ }_{\mathbf{b}^{\prime}} \dot{\mathbf{K}}_{\mathbf{a}^{\prime}}^{\mathfrak{c}} \otimes{ }_{\mathbf{b}^{\prime \prime}} \dot{\mathbf{K}}_{\mathbf{a}^{\prime \prime}}, \\
& \Delta_{\mathbf{b}^{\prime}, \mathbf{a}^{\prime}, \mathbf{b}^{\prime \prime}, \mathbf{a}^{\prime \prime}}^{\mathfrak{c}}([A])=\sum_{i, j} C_{i, j}(v, 1)\left[A_{i}^{\prime}\right] \otimes{ }^{\mathfrak{a}}\left[A_{j}^{\prime \prime}\right],
\end{aligned}
$$

where $C_{i, j}\left(v, v^{\prime}\right), A_{i}^{\prime}$ and $A_{j}^{\prime \prime}$ are as in Proposition 9.3.4. We shall call the collection

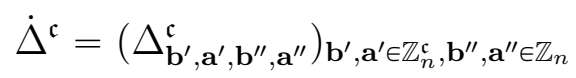

the comultiplication of $\dot{\mathbf{K}}_{n}^{\mathfrak{c}}$. Let $g_{A, B}^{C}$ and $h_{A}^{B, C}$ be the structure constants of the multiplication and comultiplication of $\dot{\mathbf{K}}_{n}^{\mathfrak{c}}$, respectively, with respect to the standard bases.

Proposition 9.6.1. The comultiplication $\dot{\Delta}^{\mathfrak{c}}$ on $\dot{\mathbf{K}}_{n}^{\mathfrak{c}}$ is an algebra homomorphism in the following sense: for all matrices $A, B, C^{\prime} \in \widetilde{\Xi}_{n}, C^{\prime \prime} \in \widetilde{\Theta}_{n}$ one has

$$
\sum_{C \in \widetilde{\Xi}_{n}} g_{A, B}^{C} h_{C}^{C^{\prime}, C^{\prime \prime}}=\sum_{A^{\prime}, B^{\prime} \in \widetilde{\Xi}_{n}, A^{\prime \prime}, B^{\prime \prime} \in \widetilde{\Theta}_{n}} h_{A}^{A^{\prime}, A^{\prime \prime}} h_{B}^{B^{\prime}, B^{\prime \prime}} g_{A^{\prime}, B^{\prime}}^{C^{\prime}} g_{A^{\prime \prime}, B^{\prime \prime}}^{C^{\prime \prime}}
$$

Proof. The proof is the same as that of Proposition 9.6.1, and shall not be repeated.

Proposition 9.6.2 can be equivalently presented in terms of the following commutative diagram: for all sequences $\mathbf{a}, \mathbf{a}^{\prime}, \mathbf{b}, \mathbf{b}^{\prime}, \mathbf{c} \in \mathbb{Z}_{n}^{\mathbf{c}}$ and $\mathbf{a}^{\prime \prime}, \mathbf{b}^{\prime \prime} \in \mathbb{Z}_{n}$ such that $\mathbf{a}^{\prime}+\mathbf{a}^{\prime \prime} \models \mathbf{a}$ and $\mathbf{b}^{\prime}+\mathbf{b}^{\prime \prime} \models \mathbf{b}$, we have

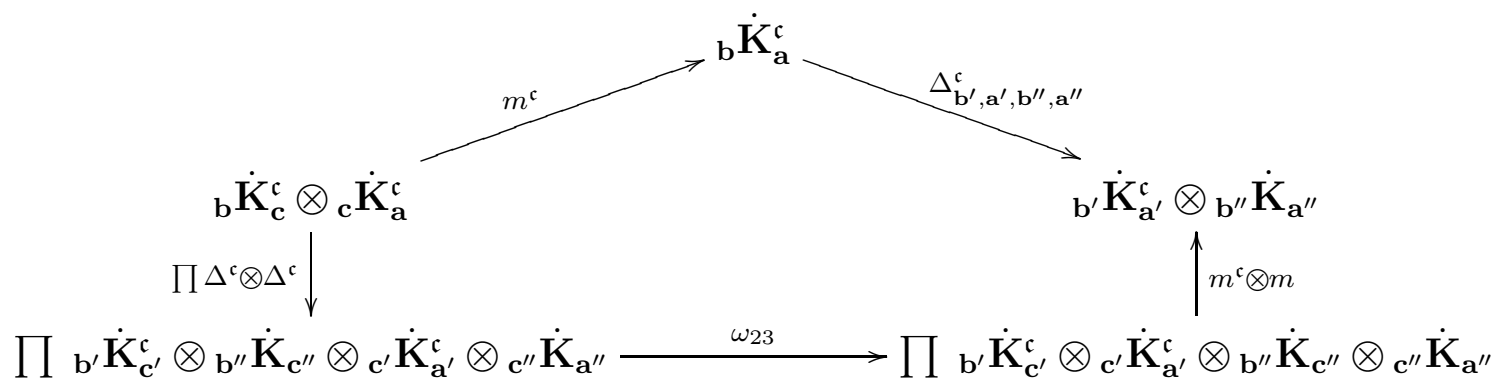

Here $m^{\mathfrak{c}}$ stands for the multiplication in $\dot{\mathbf{K}}_{n}^{\mathfrak{c}}$, all products run over all sequences $\mathbf{c}^{\prime} \in \mathbb{Z}_{n}^{\mathfrak{c}}$ and $\mathbf{c}^{\prime \prime} \in \mathbb{Z}_{n}$ such that $\mathbf{c}^{\prime}+\mathbf{c}^{\prime \prime}=\mathbf{c}$, and $\prod \Delta^{\mathfrak{c}} \otimes \Delta^{\mathfrak{c}}$ stands for the product of $\Delta_{\mathbf{b}^{\prime}, \mathbf{c}^{\prime}, \mathbf{b}^{\prime \prime}, \mathbf{c}^{\prime \prime}}^{\mathfrak{c}} \otimes \Delta_{\mathbf{c}^{\prime}, \mathbf{a}^{\prime}, \mathbf{c}^{\prime \prime}, \mathbf{a}^{\prime \prime}}$

Proposition 9.6.2. The comultiplication $\dot{\Delta}^{\mathfrak{c}}$ is coassociative in the following sense: for any matrices $A, A^{\prime} \in \widetilde{\Xi}_{n}, A^{\prime \prime}, A^{\prime \prime \prime} \in \widetilde{\Theta}_{n}$, we have

$$
\sum_{C \in \widetilde{\Xi}_{n}} h_{A}^{C, A^{\prime \prime \prime}} h_{C}^{A^{\prime}, A^{\prime \prime}}=\sum_{B \in \widetilde{\Theta}_{n}} h_{A}^{A^{\prime}, B}{ }^{a} h_{B}^{A^{\prime \prime}, A^{\prime \prime \prime}} .
$$

Proof. The proof is similar to that of Proposition 9.5.4, where we use Proposition 9.3.2 instead of Remark 9.3.3.

Proposition 9.6.2 can be equivalently formulated as the following commutative diagram: for $\tilde{\mathbf{a}}, \mathbf{a}, \mathbf{a}^{\prime}, \tilde{\mathbf{b}}, \mathbf{b}, \mathbf{b}^{\prime} \in \mathbb{Z}_{n}^{\mathbf{c}}, \mathbf{a}^{\prime \prime}, \mathbf{a}^{\prime \prime \prime}, \mathbf{b}^{\prime \prime}, \mathbf{b}^{\prime \prime \prime} \in \mathbb{Z}_{n}$ such that $\left(\mathbf{a}^{\prime}, \mathbf{a}^{\prime \prime}\right) \models \mathbf{a},\left(\mathbf{b}^{\prime}, \mathbf{b}^{\prime \prime}\right) \models \mathbf{b},\left(\mathbf{a}, \mathbf{a}^{\prime \prime \prime}\right) \models \tilde{\mathbf{a}}$ 
and $\left(\mathbf{b}, \mathbf{b}^{\prime \prime \prime}\right) \models \tilde{\mathbf{b}}$, we have

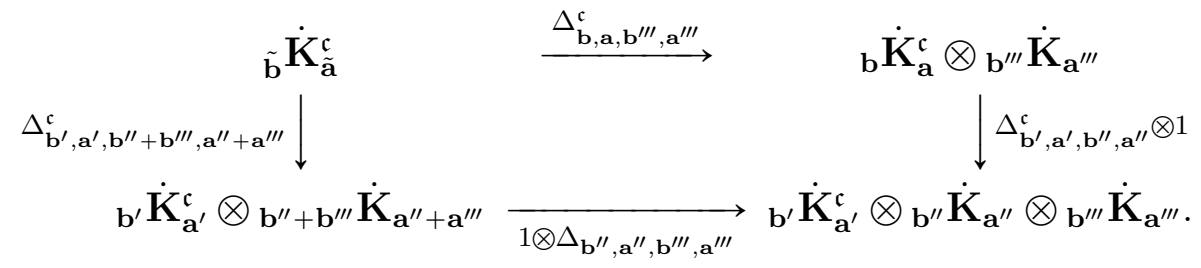

Remark 9.6.3. In light of Propositions 9.6.1 and 9.6.2, we say that the pair $\left(\dot{\mathbf{K}}_{n}, \dot{\mathbf{K}}_{n}^{\mathfrak{c}}\right)$ forms an idempotented quantum symmetric pair. Recall from Remark 9.5 .2 that $\dot{\mathbf{K}}_{n}$ is isomorphic to the idempotented quantum affine $\mathfrak{g l}_{n}, \dot{\mathbf{U}}\left(\hat{\mathfrak{g l}}_{n}\right)$.

\subsection{A hOMOMORPHiSM FROM $\dot{\mathbf{K}}_{n}^{\mathfrak{c}}$ TO $\mathbf{S}_{n, d}^{\mathfrak{c}}$}

Recall that we set $[A]_{d}=0$ and $\mathbf{f}_{A ; d}=0$ in $\mathbf{S}_{n, d}^{\mathfrak{c}}$ if $A \notin \Xi_{n, d}$. We define a linear map

$$
\begin{aligned}
& \Psi_{n, d}: \dot{\mathbf{K}}_{n}^{\mathfrak{c}} \longrightarrow \mathbf{S}_{n, d}^{\mathfrak{c}}, \\
& {[A] \mapsto[A]_{d}, \quad \text { for } A \in \widetilde{\Xi}_{n} .}
\end{aligned}
$$

Lemma 9.7.1. For all $A \in \widetilde{\Xi}_{n}$, we have $\Psi_{n, d}\left(\mathbf{f}_{A}\right)=\mathbf{f}_{A ; d}$. In particular, the map $\Psi_{n, d}$ commutes with the bar involutions.

Proof. By Proposition 9.2.3, we have

$$
\mathbf{f}_{A ; d}=\sum_{\mathbf{t} \in \mathcal{T}_{\mathbf{i}, \mathbf{a}, D_{c(\ddot{A})}}} Q_{\mathbf{i}, \mathbf{a} ; D_{c(\ddot{A})}}^{\mathbf{t}}(v, 1)\left[\operatorname{dlt}_{1}\left(D_{\operatorname{co}(\ddot{A})}\right)_{\mathbf{i}, \mathbf{a}, \mathbf{t}}\right]_{d} .
$$

The equality $\Psi_{n, d}\left(\mathbf{f}_{A}\right)=\mathbf{f}_{A ; d}$ follows readily by comparing (9.7.1) and (9.4.2).

Since $\mathbf{f}_{A}$ and $\mathbf{f}_{A ; d}$ are bar-invariant, it follows that $\Psi_{n, d}$ commutes with the bar maps.

Proposition 9.7.2. The map $\Psi_{n, d}$ is a surjective algebra homomorphism.

Proof. By Theorem 9.4.6 and Lemma 9.7.1, it suffices to show that

$$
\Psi_{n, d}\left(\mathbf{f}_{A_{1}} \cdot \mathbf{f}_{A_{2}}\right)=\mathbf{f}_{A_{1} ; d} * \mathbf{f}_{A_{2} ; d}, \quad \forall A_{1}, A_{2} \in \widetilde{\Xi}_{n} .
$$

Let $\left(\mathbf{i}_{1}, \mathbf{a}_{1}\right)$ and $\left(\mathbf{i}_{2}, \mathbf{a}_{2}\right)$ be the pairs of tuples associated to $\mathbf{f}_{A_{1}}$ and $\mathbf{f}_{A_{2}}$, respectively, in (9.4.2). The product $\mathbf{f}_{A_{1}} \cdot \mathbf{f}_{A_{2}}$ can then be written in a similar form as (9.4.2) with (i, a) replaced by $\left(\mathbf{i}_{1} \mathbf{i}_{2}, \mathbf{a}_{1} \mathbf{a}_{2}\right)$, by Proposition 9.4.4. Similarly, the product $\mathbf{f}_{A_{1} ; d} * \mathbf{f}_{A_{2} ; d}$ admits a similar form of (9.7.1) with $(\mathbf{i}, \mathbf{a})$ replaced by $\left(\mathbf{i}_{1} \mathbf{i}_{2}, \mathbf{a}_{1} \mathbf{a}_{2}\right)$. By arguing in a similar fashion as the proof of Lemma 9.7.1, we see that $\Psi_{n, d}$ sends the product $\mathbf{f}_{A_{1}} \cdot \mathbf{f}_{A_{2}}$ to $\mathbf{f}_{A_{1} ; d} * \mathbf{f}_{A_{2} ; d}$.

By a standard argument such as the proof of [BKLW14, Theorem A.21], we reach at the following result.

Theorem 9.7.3. We have $\Psi_{n, d}(\{A\})=\{A\}_{d}$ if $A \in \Xi_{n, d}$, and zero otherwise. 


\subsection{The Algebra $\dot{\mathbf{K}}_{n}^{\mathfrak{c}}$ AS A SUbquotient OF $\dot{\mathbf{K}}_{\breve{n}}^{\mathfrak{c}}$}

Let $\dot{\mathbf{K}}_{\breve{n}, 1,0}^{\mathfrak{c}}$ be the subalgebra of $\dot{\mathbf{K}}_{\breve{n}}^{\mathfrak{c}}$ spanned by the elements $[A]$ such that $\operatorname{ro}(A)_{1}=$ $\operatorname{co}(A)_{1}=0$. Let $\mathcal{I}$ be the subspace of $\dot{\mathbf{K}}_{\breve{n}, 1,0}^{\mathfrak{c}}$ spanned by those elements $[A]$ such that $a_{1,1}<0$. Then a similar argument for Lemma 9.7.1 (see also [BLW14, A.3]) gives us the following.

Lemma 9.8.1. The subspace $\mathcal{I}$ is a two-sided ideal of $\dot{\mathbf{K}}_{\breve{n}, 1,0}^{\mathfrak{c}}$ and $\mathcal{I} \cap\left\{\{A\} \mid A \in \widetilde{\Xi}_{\breve{n}}\right\}$ forms a basis of $\mathcal{I}$.

Let $\dot{\mathbf{K}}_{\breve{n}, 1,0}^{\mathfrak{c}} / \mathcal{I}$ be the quotient algebra of $\dot{\mathbf{K}}_{\tilde{n}, 1,0}^{\mathfrak{c}}$ by $\mathcal{I}$. By Lemma 9.8.1, the set

$$
\left\{\{A\}+\mathcal{I} \mid A \in \widetilde{\Xi}_{\breve{n}}, a_{1, i}=a_{i, 1}=0, \forall i \in \mathbb{Z}\right\}
$$

is a stably canonical basis of $\dot{\mathbf{K}}_{\breve{n}, 1,0}^{\mathfrak{c}} / \mathcal{I}$. We shall identify the subquotient $\dot{\mathbf{K}}_{\tilde{n}, 1,0}^{\mathfrak{c}} / \mathcal{I}$ with the stabilization algebra $\dot{\mathbf{K}}_{n}^{\mathrm{c}}$.

Proposition 9.8.2. The assignment $\tilde{\rho}:[A] \mapsto[\ddot{A}]+\mathcal{I}$, for all $A \in \widetilde{\Xi}_{n}$, defines an isomorphism from the algebra $\dot{\mathbf{K}}_{n}^{\mathrm{c}}$ to the subquotient $\dot{\mathbf{K}}_{\tilde{n}, 1,0}^{\mathfrak{c}} / \mathcal{I}$ of $\dot{\mathbf{K}}_{\breve{n}}^{\mathfrak{c}}$ with compatible stably canonical bases.

Proof. By a similar argument as in the proof of Lemma 9.7.1, we have

$$
\tilde{\rho}\left(\mathbf{f}_{A}\right)=\mathbf{f}_{\ddot{A}}+\mathcal{I}, \quad \forall A \in \widetilde{\Xi}_{n} .
$$

A similar argument as in the proof of Proposition 9.7.2 shows that $\tilde{\rho}$ is an algebra homomorphism by showing that $\rho\left(\mathbf{f}_{A_{1}} \cdot \mathbf{f}_{A_{2}}\right)=\mathbf{f}_{\ddot{A}_{1}} \cdot \mathbf{f}_{\ddot{A}_{2}}+\mathcal{I}$ for all $A_{1}, A_{2} \in \widetilde{\Xi}_{n}$. By Lemma 9.8.1 we know that $\tilde{\rho}$ is an algebra isomorphism. A standard argument shows the compatibility with the canonical bases. The proposition is thus proved.

Clearly, the projection $\Psi_{\breve{n}, d}: \dot{\mathbf{K}}_{\breve{n}}^{c} \rightarrow \mathbf{S}_{\breve{n}, d}^{\mathfrak{c}}$ induces a projection $\Psi_{\breve{n}, d}: \dot{\mathbf{K}}_{\breve{n}, 1,0}^{\mathfrak{c}} / \mathcal{I} \rightarrow \mathbf{S}_{\breve{n}, d}^{\mathfrak{c}}$. We have the following commutative diagram:

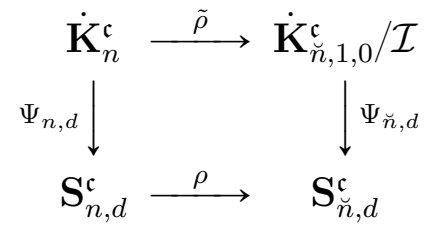

Remark 9.8.3. The construction of $\dot{\mathbf{K}}_{n}^{\mathfrak{c}}$ as a subquotient of $\dot{\mathbf{K}}_{\breve{n}}^{\mathfrak{c}}$ here is modeled on the construction in [BLW14] (see also [FL14]), where an algebra $\dot{\mathbf{U}}^{\imath}$ is realized as a subquotient of an algebra $\dot{\mathbf{U}}^{\jmath}$ with compatible stably canonical bases. 


\section{CHAPTER 10}

\section{Stabilization algebras arising from other Schur algebras}

In this chapter, the approach to the stabilization of the family of Schur algebras $\mathbf{S}_{n, d}^{\mathfrak{c}}$ (as $d$ varies) in the preceding Chapter 9 will be adapted with modifications to study the remaining 3 families of Schur algebras of types $\jmath$, $\imath$ and $\imath \imath$. We will present more details for the type $\jmath$ while merely formulating the main statements for types $\imath \jmath$ and $\imath$.

\subsection{A mONOMial BASIS FOR Schur ALGEBRA $\mathbf{S}_{\mathfrak{n}, d}^{\jmath \imath}$}

Recall that $\mathfrak{n}=n-1=2 r+1$. Recall the set $\Xi_{\mathfrak{n}, d}^{\jmath \imath}$ from (7.1.1), the set $\check{\Xi}_{\mathfrak{n}, d}^{\jmath}$ from (7.3.2), and the bijection from (7.3.3)

$$
\operatorname{dlt}_{r+1}: \Xi_{\mathfrak{n}, d}^{\jmath \imath} \longrightarrow \check{\Xi}_{\mathfrak{n}, d}^{\jmath 2}
$$

We also set $\ddot{A}=\operatorname{dlt}_{r+1}^{-1}(A)$ for all $A \in \check{\Xi}_{\mathfrak{n}, d}^{\jmath}$.

Recall the subalgebra $\mathbf{S}_{\mathfrak{n}, d}^{\jmath n}$ of $\mathbf{S}_{n, d}^{\mathfrak{c}}$ from (7.1.3). Since the comultiplication $\Delta^{\mathfrak{c}}$ on $\mathbf{S}_{n, d}^{\mathfrak{c}}$ is coassociative, so is the comultiplication $\Delta^{\jmath \imath}$ on $\mathbf{S}_{\mathfrak{n}, d}^{\jmath \imath}$.

For each tridiagonal matrix $A \in \check{\Xi}_{\mathfrak{n}, d}^{\mathfrak{n}}$ such that $\operatorname{dlt}_{r+1}(A)-\sum_{1 \leqslant i \leqslant \mathfrak{n}} \alpha_{i} E_{\theta, \mathfrak{n}}^{i, i+1}$ is diagonal, we define

$$
\mathbf{f}_{A ; d}^{\jmath \imath}=\mathbf{f}_{r}^{\left(\alpha_{r}\right)} * \mathbf{f}_{r-1}^{\left(\alpha_{r-1}\right)} * \cdots * \mathbf{f}_{-(r+1)}^{\left(\alpha_{-(r+1)}\right)} * 1_{\mathrm{co}(A)} \in \mathbf{S}_{\mathfrak{n}, d}^{\jmath \imath}
$$

We call a matrix $A \in \Xi_{\mathfrak{n}, d}^{\jmath} \jmath$-tridiagonal, if the associated matrix $\operatorname{dlt}_{r+1}(A)$ is tridiagonal. Given any matrix $A=\left(a_{i j}\right)$ in $\Xi_{\mathfrak{n}, d}^{\jmath n}$ of depth $m \geqslant 1$ and $\operatorname{dlt}_{r+1}(A)=\left(a_{i j}^{\prime}\right)$, we define $\jmath^{\prime-}$ tridiagonal matrices $A_{1}, A_{2}, \ldots, A_{m} \in \Xi_{\mathfrak{n}, d}^{\jmath n}$ by the conditions that $\operatorname{ro}\left(A_{m}\right)=\operatorname{ro}(A), \operatorname{co}\left(A_{1}\right)=$ $\operatorname{co}(A), \operatorname{ro}\left(A_{i}\right)=\operatorname{co}\left(A_{i+1}\right)$ for $1 \leqslant i \leqslant m-1$ and $\operatorname{dlt}_{r+1}\left(A_{i}\right)-\sum_{1 \leqslant j \leqslant n}\left(\sum_{k \leqslant j-i+1} a_{k, j+1}^{\prime}\right) E_{\theta, \mathfrak{n}}^{j, j+1}$ is diagonal for all $1 \leqslant i \leqslant m$. Then we set

$$
\mathbf{f}_{A ; d}^{\jmath \imath}=\mathbf{f}_{A_{m} ; d}^{\jmath \imath} * \mathbf{f}_{A_{m-1} ; d}^{\jmath \imath} * \cdots * \mathbf{f}_{A_{1} ; d}^{\jmath \imath} .
$$

By definition, the element $\mathbf{f}_{A ; d}^{\jmath \imath}$ is bar-invariant.

By an argument similar to Theorem 9.1.6, we have the following.

Proposition 10.1.1. (1) We have $\mathbf{f}_{A ; d}^{\jmath \mathfrak{l}}=[A]_{d}+$ lower terms, for all $A \in \Xi_{\mathfrak{n}, d}^{\jmath n}$.

(2) The set $\left\{\mathbf{f}_{A ; d}^{\jmath} \mid A \in \Xi_{\mathfrak{n}, d}^{\jmath}\right\}$ forms a bar-invariant basis of $\mathbf{S}_{\mathfrak{n}, d}^{\jmath}$ (called a monomial basis).

\subsection{Stabilization of Schur Algebras of type $\imath$}

Now we shall formulate the stabilization of the family of Schur algebras $\left\{\mathbf{S}_{\mathfrak{n}, d}^{\jmath \imath}\right\}_{d \geqslant 1}$, analogous to the family of Schur algebras $\left\{\mathbf{S}_{n, d}^{\mathfrak{c}}\right\}_{d \geqslant 1}$ treated in Section 9.2 .

Recall $\widetilde{\Xi}_{\mathfrak{n}, d}^{\jmath}$ in (7.4.2) is a variant of $\Xi_{\mathfrak{n}, d}^{\jmath \imath}$ which does not require the diagonal entries to be nonnegative. 
Recall the set $\widetilde{\Xi}_{n, d}$ from (6.2.3). For $0 \leqslant i \leqslant n-1, A \in \widetilde{\Xi}_{n, d}$ for all $j \in \mathbb{Z}, t=\left(t_{u}\right)_{u \in \mathbb{Z}} \in \mathbb{N}^{\mathbb{Z}}$ such that $\sum_{j \in \mathbb{Z}} t_{u}=R$, we define the polynomials $Q_{i, R ; A}^{j l, t} \in \mathscr{R}$ as follows. For any $i \in$ $[1, n-1] \backslash\{r, r+1\}$, we define

$$
Q_{i, R ; A}^{\jmath \imath, t}\left(v, v^{\prime}\right)=v^{\beta_{t}} \prod_{u \in \mathbb{Z}, u \neq i} \overline{\left[\begin{array}{c}
a_{i u}+t_{u} \\
t_{u}
\end{array}\right]} \cdot v^{\prime\left(\delta_{i, 1}+\delta_{i, n-1}\right) \sum_{i+1 \geqslant u} t_{u}} \overline{\left[\begin{array}{c}
a_{i i}+t_{i} \\
t_{i}
\end{array}\right]_{v, v^{\prime}}},
$$

where

$$
\beta_{t}=\sum_{j \geqslant u} a_{i j} t_{u}-\sum_{j>u} a_{i+1, j} t_{u}+\sum_{j<u} t_{j} t_{u}+\frac{1}{2}\left(\delta_{i, r}+\delta_{i, n-1}\right)\left(\sum_{j+u<2(i+1)} t_{j} t_{u}+\sum_{j<i+1} t_{j}\right) .
$$

We further define

$$
\begin{aligned}
& Q_{r, R ; A}^{\jmath, t}\left(v, v^{\prime}\right)=v^{\beta_{t}} \prod_{u \in \mathbb{Z}, u \neq i} \overline{\left[\begin{array}{c}
a_{i u}+t_{u} \\
t_{u}
\end{array}\right]} \cdot v^{\prime-\sum_{i \geqslant u} t_{u}}, \quad \text { for } i=r \\
& Q_{r+1, R ; A}^{\jmath n, t}\left(v, v^{\prime}\right)=v^{\beta_{t}^{\prime}} \prod_{u>i} \overline{\left[\begin{array}{c}
a_{i u}+t_{u}+t_{2 i-u} \\
t_{u}
\end{array}\right]} \prod_{u<i} \overline{\left[\begin{array}{c}
a_{i u}+t_{u} \\
t_{u}
\end{array}\right]} \cdot v^{\prime \sum_{i+1 \geqslant u} t_{u}}, \quad \text { for } i=r+1
\end{aligned}
$$

where

$$
\beta_{t}^{\prime}=\sum_{j \geqslant u} a_{i j} t_{u}-\sum_{j>u} a_{i+1, j} t_{u}+\sum_{j<u, j+u \leqslant 2 i} t_{j} t_{u}-\sum_{j>i} \frac{t_{j}^{2}-t_{j}}{2}+\frac{R^{2}-R}{2} .
$$

Given tuples $\mathbf{i}=\left(i_{1}, \ldots, i_{s}\right)$ and $\mathbf{a}=\left(a_{1}, \ldots, a_{s}\right) \in \mathbb{N}^{s}$ and a tuple $\mathbf{t}=\left(t_{1}, \ldots, t_{s}\right)$ satisfying (9.2.6), we defined the polynomials $Q_{\mathbf{i}, \mathbf{a} ; A}^{\mathbf{t}}$ in (9.2.7). We can similarly define the polynomials $Q_{\mathbf{i}, \mathbf{a} ; A}^{\jmath \imath, \mathbf{t}}\left(v, v^{\prime}\right)$ in $\mathscr{R}$, inductively on $s$ starting with (10.2.1)-(10.2.3), for $A \in \widetilde{\Xi}_{\mathfrak{n}, d}^{\jmath \imath}$.

Propositions 10.2.1 10.2.5 are the $\jmath$-counterparts of Propositions 9.2.3 9.2.7. We skip the similar proofs. The notations are understood in this section that $\ddot{I}_{\mathfrak{n}}=I_{n}-E_{n}^{r+1, r+1}$, and $\ddot{p} A=A+p \ddot{I}_{\mathfrak{n}}$.

Proposition 10.2.1. Assume $A, B_{j} \in \widetilde{\Xi}_{n, d}$, for $1 \leqslant j \leqslant s$, and a pair of tuples (i,a) satisfy the following properties: $\operatorname{ro}(A)=\operatorname{co}\left(B_{s}\right), \operatorname{ro}\left(B_{j}\right)=\operatorname{co}\left(B_{i-1}\right), \forall 1<i \leqslant s, B_{j}-a_{j} E_{\theta, n}^{i_{j}, i_{j}+1}$ is diagonal and $a_{r+1, j}=\delta_{j, r+1}$ for all $j \in \mathbb{Z}$. Then we have

$$
\left[\ddot{p} B_{1}\right]_{d+\frac{p}{2} \mathfrak{n}} * \cdots *\left[\ddot{p} B_{s}\right]_{d+\frac{p}{2} \mathfrak{n}} *[\ddot{p} A]_{d+\frac{p}{2} \mathfrak{n}}=\sum_{\mathbf{t} \in \mathcal{T}_{\mathbf{i}, \mathbf{a}, A}} Q_{\mathbf{i}, \mathbf{a} ; A}^{\jmath \jmath, \mathbf{t}}\left(v, v^{-p}\right)\left[\ddot{p} A_{\mathbf{i}, \mathbf{a}, \mathbf{t}}\right]_{d+\frac{p}{2} \mathfrak{n}}, \quad \forall p \in 2 \mathbb{Z} .
$$

Proposition 10.2.2. Let $A \in \widetilde{\Xi}_{\mathfrak{n}, d}^{\jmath}$. There exist $Z_{i} \in \widetilde{\Xi}_{\mathfrak{n}, d}^{\jmath}$, for $1 \leqslant i \leqslant m$, with $Z_{i}<A$, $Q_{i}\left(v, v^{\prime}\right) \in \mathscr{R}$ and $p_{0} \in \mathbb{N}$ such that

$$
[\ddot{p} A]_{d+\frac{p}{2} \mathfrak{n}}=\mathbf{f}_{\ddot{p} A ; d+\frac{p}{2} \mathfrak{n}}^{\jmath \imath}+\sum_{i=1}^{m} Q_{i}\left(v, v^{-p}\right)\left[\ddot{p} Z_{i}\right]_{d+\frac{p}{2} \mathfrak{n}}, \quad \forall p \geqslant p_{0}, p \in 2 \mathbb{N} .
$$


Proposition 10.2.3. Assume that $A_{1}, \ldots, A_{l} \in \widetilde{\Xi}_{\mathfrak{n}, d}^{\jmath n}$ satisfy $\operatorname{co}\left(A_{i}\right)=\operatorname{ro}\left(A_{i+1}\right)$ for all $1 \leqslant$ $i \leqslant l-1$. There exist $Z_{1}, \ldots, Z_{m} \in \widetilde{\Xi}_{\mathfrak{n}, d}^{n}, G_{1}\left(v, v^{\prime}\right), \ldots, G_{m}\left(v, v^{\prime}\right) \in \mathscr{R}$, and $p_{0} \in \mathbb{N}$ such that

$$
\left[\ddot{p} A_{1}\right]_{d+\frac{p}{2} \mathfrak{n}} *\left[\ddot{p} A_{2}\right]_{d+\frac{p}{2} \mathfrak{n}} * \cdots *\left[\ddot{p} A_{l}\right]_{d+\frac{p}{2} \mathfrak{n}}=\sum_{i=1}^{m} G_{i}\left(v, v^{-p}\right)\left[\ddot{p} Z_{i}\right]_{d+\frac{p}{2} \mathfrak{n}}, \quad \forall p \geqslant p_{0}, p \in 2 \mathbb{N} .
$$

Corollary 10.2.4. For any matrix $A \in \widetilde{\Xi}_{\mathfrak{n}, d}^{n \imath}$ of depth $m$ and $\operatorname{dlt}_{r+1}(A)=\left(a_{i j}^{\prime}\right)$, there exist

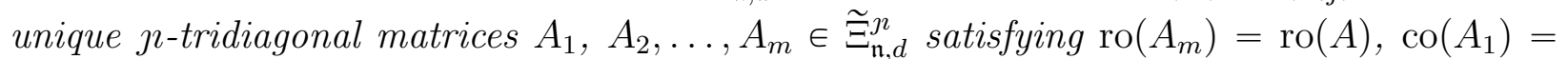
$\mathrm{co}(A), \operatorname{ro}\left(A_{i}\right)=\operatorname{co}\left(A_{i+1}\right)$ for $1 \leqslant i \leqslant m-1$ and $\operatorname{dlt}_{r+1}\left(A_{i}\right)-\sum_{1 \leqslant j \leqslant n}\left(\sum_{k \leqslant j-i+1} a_{k, j+1}^{\prime}\right) E_{\theta, \mathfrak{n}}^{j, j+1}$ is diagonal for all $1 \leqslant i \leqslant m$ such that

$\left[\ddot{p} A_{m}\right]_{d+\frac{p}{2} \mathfrak{n}} *\left[\ddot{p} A_{m-1}\right]_{d+\frac{p}{2} \mathfrak{n}} * \cdots *\left[\ddot{p} A_{1}\right]_{d+\frac{p}{2} \mathfrak{n}}=[\ddot{p} A]_{d+\frac{p}{2} \mathfrak{n}}+\sum_{i=1}^{l} G_{i}\left(v, v^{-p}\right)\left[\ddot{p} Z_{i}\right]_{d+\frac{p}{2} \mathfrak{n}}, \forall p \in 2 \mathbb{N}, p \geqslant p_{0}$, where $p_{0}, G_{i}\left(v, v^{\prime}\right) \in \mathscr{R}$ and $Z_{1}, \ldots, Z_{l} \in \widetilde{\Xi}_{\mathfrak{n}, d}^{\jmath}$ are given in Proposition 10.2.3 such that $Z_{i}<A$.

Proposition 10.2.5. Assume that $A \in \widetilde{\Xi}_{\mathfrak{n}, d}^{\jmath n}$. Then there exist $Y_{i} \in \widetilde{\Xi}_{\mathfrak{n}, d}^{\jmath \imath}$ with $Y_{i}<A$, $H_{i}\left(v, v^{\prime}\right) \in \mathscr{R}$ for all $1 \leqslant i \leqslant s$ and $p_{0} \in \mathbb{N}$ such that

$$
\overline{[\ddot{p} A}_{d+\frac{p}{2} \mathfrak{n}}=[\ddot{p} A]_{d+\frac{p}{2} \mathfrak{n}}+\sum_{i=1}^{s} H_{i}\left(v, v^{-p}\right)\left[\ddot{p} Y_{i}\right]_{d+\frac{p}{2} \mathfrak{n}}, \quad \forall p \geqslant p_{0}, p \in 2 \mathbb{N} .
$$

The following is a counterpart of Proposition 9.3.4.

Proposition 10.2.6. Assume that $d^{\prime}+d^{\prime \prime}=d$ and that $\mathbf{b}^{\prime}, \mathbf{a}^{\prime} \in \mathbb{Z}_{n}^{\mathfrak{c}}$ and $\mathbf{b}^{\prime \prime}, \mathbf{a}^{\prime \prime} \in \mathbb{Z}_{\mathfrak{n}}$ so that ${ }_{p} \Delta_{\mathbf{b}^{\prime}, \mathbf{a}^{\prime}, \mathbf{b}^{\prime \prime}, \mathbf{a}^{\prime \prime}}^{\jmath s}$ defined. Let $A \in \widetilde{\Xi}_{\mathfrak{n}, d}^{n}$. There exist $A_{i}^{\prime} \in \widetilde{\Xi}_{\mathfrak{n}, d^{\prime}}^{\jmath}$ where $1 \leqslant i \leqslant l$ for some $l$, $A_{j}^{\prime \prime} \in \widetilde{\Theta}_{\mathfrak{n}, d^{\prime \prime}}^{\jmath n}$ where $1 \leqslant j \leqslant m$ for some $m, C_{i, j}\left(v, v^{\prime}\right) \in \mathscr{R}$ for $1 \leqslant i \leqslant l, 1 \leqslant j \leqslant m$, and $p_{0} \in \mathbb{N}$ such that

$$
\ddot{p} \Delta_{\mathbf{b}^{\prime}, \mathbf{a}^{\prime}, \mathbf{b}^{\prime \prime}, \mathbf{a}^{\prime \prime}}\left([\ddot{2 p} A]_{d+p \mathfrak{n}}\right)=\sum_{1 \leqslant i \leqslant l, 1 \leqslant j \leqslant m} C_{i, j}\left(v, v^{-p}\right)\left[\ddot{p} A_{i}^{\prime}\right]_{d^{\prime}+\frac{p}{2} \mathfrak{n}} \otimes{ }^{\mathfrak{a}}\left[\ddot{p} A_{j}^{\prime \prime}\right]_{d^{\prime \prime}+p \mathfrak{n}}, \quad \forall p \geqslant p_{0}, p \in 2 \mathbb{N} \text {. }
$$

\subsection{The Stabilization ALGEBRA $\dot{\mathbf{K}}_{n}^{\jmath \imath}$}

Recall the set $\widetilde{\Xi}_{\mathfrak{n}}^{\jmath}$ and $\widetilde{\Xi}_{\mathfrak{n}, d}^{\jmath}$ from (7.4.2). Consider the $\mathbb{Q}(v)$-space $\dot{\mathbf{K}}_{\mathfrak{n}}^{\jmath}$ spanned by the formal symbols $[A]$ for all $A \in \widetilde{\Xi}_{\mathfrak{n}}^{\jmath n}$. We define an associative algebra structure on $\dot{K}_{\mathfrak{n}}^{\jmath n}$ by

$$
\left[A_{1}\right] \cdot\left[A_{2}\right]=\sum_{i=1}^{m} G_{i}(v, 1)\left[Z_{i}\right], \quad \forall A_{1}, A_{2} \in \widetilde{\Xi}_{\mathfrak{n}}^{\mathfrak{n}},
$$

where $G_{i}\left(v, v^{\prime}\right) \in \mathscr{R}$ and $Z_{i}$ are from Proposition 10.2.3.

For each $A \in \widetilde{\Xi}_{\mathfrak{n}}^{\jmath}$, we define

$$
\mathbf{f}_{A}^{\jmath \imath}=[A]+\sum_{i_{1}}^{m} G_{i}(v, 1)\left[Z_{i}\right],
$$

where $G_{i}\left(v, v^{\prime}\right) \in \mathscr{R}$ and $Z_{i}$ are from Corollary 10.2.4. It follows by definition that $\left\{\mathbf{f}_{A}^{\jmath \imath} \mid A \in\right.$ $\left.\widetilde{\Xi}_{\mathfrak{n}}^{\jmath}\right\}$ forms a basis of $\dot{\mathbf{K}}_{\mathfrak{n}}^{\jmath \imath}$ (called a monomial basis).

By Proposition 10.2.1, we can establish the following. 
Proposition 10.3.1. (1) For any $A \in \widetilde{\Xi}_{\mathfrak{n}}^{\jmath n}$, there exists a pair $(\mathbf{i}, \mathbf{a})$ of tuples such that

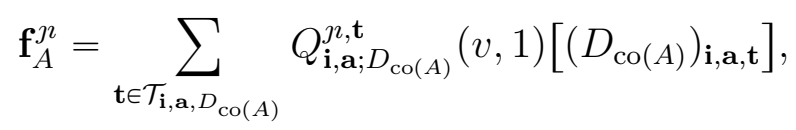

where $D_{\operatorname{co}(A)}$ is the diagonal matrix in $\widetilde{\Xi}_{\mathfrak{n}}^{\mathfrak{n}}$ with diagonal $\operatorname{co}(A)$.

(2) The element $\mathbf{f}_{A}^{\jmath \imath}$ can be written in a product form as

$$
\mathbf{f}_{A}^{\jmath \imath}=\mathbf{f}_{A_{m}}^{\jmath \imath} \cdot \mathbf{f}_{A_{m-1}}^{\jmath \imath} \cdot \ldots \cdot \mathbf{f}_{A_{1}}^{\jmath \imath}
$$

where $A_{i}$ are $u$-tridiagonal matrices defined similarly as in (9.4.4).

(3) We have $\overline{\mathbf{f}_{A}^{\jmath \imath}}=\mathbf{f}_{A}^{\jmath \imath}$, for $A \in \widetilde{\Xi}_{\mathfrak{n}}^{\jmath \imath}$.

Similarly, for $A \in \widetilde{\Xi}_{\mathfrak{n}}^{\jmath}$, we set

$$
m_{A}^{\jmath \imath}=\left[A_{m}\right] \cdot\left[A_{m-1}\right] \cdots\left[A_{1}\right]
$$

where $A_{i}$ 's are the same as in (10.3.4). One also has $m_{A}^{\jmath \imath}=\mathbf{f}_{A}^{\jmath \imath}+$ lower terms. Thus $\left\{m_{A}^{\jmath \imath} \mid A \in \widetilde{\Xi}_{\mathfrak{n}}^{\jmath n}\right\}$ forms a basis for $\dot{\mathbf{K}}_{\mathfrak{n}}^{\jmath \imath}$ (called a semi-monomial basis). Just like its J-sibling, the monomial $m_{A}^{\jmath}$ is not necessarily bar-invariant.

The following multiplication formula on $\dot{\mathbf{K}}_{\mathfrak{n}}^{\jmath \imath}$ follows from Proposition 10.2.1,

Proposition 10.3.2. Assume the matrices $A, B \in \widetilde{\Xi}_{\mathfrak{n}}^{n}$ satisfy that $\operatorname{co}(B)=\operatorname{ro}(A)$ and $\operatorname{dlt}_{r+1}(B)-\sum_{1 \leqslant i \leqslant n} \beta_{i} E_{\theta, \mathfrak{n}}^{i, i+1}$ is diagonal. Then we have a multiplication formula of the form

$$
\mathbf{f}_{B}^{\jmath \imath} \cdot[A]=\sum_{\mathbf{t} \in \mathcal{T}_{\mathbf{i}_{0}^{\jmath}, \mathbf{b}_{0}^{j n}, A}} Q_{\mathbf{i}_{0}^{j \imath}, \mathbf{b}_{0}^{j \imath} ; A}^{\jmath, \mathbf{t}}(v, 1)\left[A_{\mathbf{i}_{0}^{j \imath}, \mathbf{b}_{0}^{\jmath n}, \mathbf{t}}\right],
$$

where $\mathbf{i}_{0}^{\jmath \imath}=(r, r-1, \ldots, 1-r)$ and $\mathbf{b}_{0}^{\jmath \imath}=\left(\beta_{r}, \beta_{r-1}, \ldots, \beta_{-r}\right)$.

We define a bar involution on $\dot{\mathbf{K}}_{\mathfrak{n}}^{\mathfrak{n}}$ by

$$
\overline{[A]}=[A]+\sum_{i=1}^{s} H_{i}(v, 1)\left[Y_{i}\right], \quad \forall A \in \widetilde{\Xi}_{\mathfrak{n}}^{\jmath},
$$

where $H_{i}\left(v, v^{\prime}\right)$ and $Y_{i}<A$ are from Proposition 10.2.5. By a standard argument, we can now establish the existence of the stably canonical basis for $\dot{\mathbf{K}}_{\mathfrak{n}}^{j n}$.

Proposition 10.3.3. (1) For any $A \in \widetilde{\Xi}_{\mathfrak{n}}^{\jmath}$, there exists a unique element $\{A\}$ in $\dot{\mathbf{K}}_{\mathfrak{n}}^{\jmath n}$ such that

$$
\overline{\{A\}}=\{A\}, \quad\{A\}=[A]+\sum_{A^{\prime}<A} \pi_{A, A^{\prime}}^{\jmath}\left[A^{\prime}\right], \quad \pi_{A, A^{\prime}}^{\jmath} \in v^{-1} \mathbb{Z}\left[v^{-1}\right]
$$

(2) The set $\left\{\{A\} \mid A \in \widetilde{\Xi}_{\mathfrak{n}}^{\jmath}\right\}$ forms a basis for $\dot{\mathbf{K}}_{\mathfrak{n}}^{\jmath n}$ (called the stably canonical basis).

Let us summarize the main results of this section as follows.

Theorem 10.3.4. The algebra $\dot{\mathbf{K}}_{\mathfrak{n}}^{\jmath \imath}$ admits a standard basis $\left\{[A] \mid A \in \widetilde{\Xi}_{\mathfrak{n}}^{\jmath n}\right\}$, a semi-monomial basis $\left\{m_{A}^{\jmath} \mid A \in \widetilde{\Xi}_{\mathfrak{n}}^{\jmath}\right\}$, a monomial basis $\left\{\mathbf{f}_{A}^{\jmath \imath} \mid A \in \widetilde{\Xi}_{\mathfrak{n}}^{\jmath \imath}\right\}$, and a canonical basis $\left\{\{A\} \mid A \in \widetilde{\Xi}_{\mathfrak{n}}^{\jmath\urcorner}\right\}$.

Recall our convention that $[A]_{d}=0$ in $\mathbf{S}_{\mathfrak{n}, d}^{\jmath}$, for all $A \in \widetilde{\Xi}_{\mathfrak{n}}^{\jmath\urcorner} \Xi_{\mathfrak{n}, d}^{\jmath \imath}$. The following is a counterpart of Theorem 9.7.3. 
Theorem 10.3.5. The assignment $[A] \mapsto[A]_{d}$, for all $A \in \widetilde{\Xi}_{\mathfrak{n}}^{\jmath}$, defines a surjective algebra homomorphism $\Psi_{\mathfrak{n}, d}^{\jmath \imath}: \dot{\mathbf{K}}_{\mathfrak{n}}^{\jmath \imath} \rightarrow \mathbf{S}_{\mathfrak{n}, d}^{\jmath \imath}$. Moreover, we have $\Psi_{\mathfrak{n}, d}^{\jmath \imath}(\{A\})=\{A\}_{d}$ if $A \in \Xi_{\mathfrak{n}, d}^{\jmath \imath}$ and zero otherwise.

We have developed the current Chapter 10 on the stabilization algebra $\dot{\mathbf{K}}_{\mathfrak{n}}^{\jmath}$ which is based on the imbeddings $\mathbf{S}_{\mathfrak{n}, d}^{\jmath \imath} \rightarrow \mathbf{S}_{n, d}^{\mathfrak{c}}$, in analogy to the stabilization algebra $\dot{\mathbf{K}}_{n}^{\mathfrak{c}}$ in Chapter 9 which was based on the imbeddings $\mathbf{S}_{n, d}^{\mathfrak{c}} \rightarrow \mathbf{S}_{\breve{n}, d}^{\mathfrak{c}}$. Just as the imbeddings $\mathbf{S}_{n, d}^{\mathfrak{c}} \rightarrow \mathbf{S}_{\breve{n}, d}^{\mathfrak{c}}$ lead to a realization of $\dot{\mathbf{K}}_{n}^{\mathfrak{c}}$ as a subquotient of $\dot{\mathbf{K}}_{\breve{n}}^{\mathfrak{c}}$ (see Proposition 9.8.2), the imbeddings $\mathbf{S}_{\mathfrak{n}, d}^{\jmath \imath} \rightarrow \mathbf{S}_{n, d}^{\mathfrak{c}}$ lead to a realization of $\dot{\mathbf{K}}_{\mathfrak{n}}^{\jmath \imath}$ as a subquotient of $\dot{\mathbf{K}}_{n}^{\mathfrak{c}}$.

We shall simply formulate the statement below and skip the detail (compare with [BLW14]). Let $\mathcal{J}_{<}^{\jmath}$ be the $\mathbb{Q}(v)$-subspace of $\dot{\mathbf{K}}_{n}^{\mathfrak{c}}$ spanned by $[A]$ for $A=\left(a_{i j}\right) \in \widetilde{\Xi}_{\mathfrak{n}}^{\jmath \imath}$ with $a_{r+1, r+1}<0$. Then one shows that $\mathcal{J}_{<}^{\jmath \imath}$ is a two-sided ideal of $\dot{\mathbf{K}}_{n}^{\mathfrak{c}}$ with a stably canonical basis

$$
\left\{\{A\} \mid A=\left(a_{i j}\right) \in \widetilde{\Xi}_{\mathfrak{n}}^{\jmath \imath}, a_{r+1, r+1}<0\right\} .
$$

Moreover, the natural linear map

$$
\dot{\mathbf{K}}_{\mathfrak{n}}^{\jmath \imath} \longrightarrow \dot{\mathbf{K}}_{n}^{\mathfrak{c}} / \mathcal{J}_{<}^{\jmath \imath}, \quad[A] \mapsto[A]+\mathcal{J}_{<}^{\jmath \imath}
$$

is an algebra isomorphism, and it preserves the stably canonical bases. We summarize these as follows.

Theorem 10.3.6. The algebra $\dot{\mathbf{K}}_{\mathfrak{n}}^{\mathfrak{n}}$ is a subquotient of the algebra $\dot{\mathbf{K}}_{n}^{\mathfrak{c}}$ with compatible stably canonical bases.

We finally discuss the comultiplication on $\dot{\mathbf{K}}_{\mathfrak{n}}^{\mathfrak{n}}$. Let

$$
\mathbb{Z}_{\mathfrak{n}}^{j n}=\left\{\lambda=\left(\lambda_{i}\right)_{i \in \mathbb{Z}} \in \mathbb{Z}_{n}^{\mathfrak{c}} \mid \lambda_{r+1}=1\right\}, \quad \mathbb{Z}_{\mathfrak{n}}^{\jmath \imath \mathfrak{a}}=\left\{\lambda \in \mathbb{Z}_{n} \mid \lambda_{r+1}=0\right\} .
$$

Note that there is a canonical bijection $\mathbb{Z}_{\mathfrak{n}}^{\jmath n} \simeq \mathbb{Z}_{\mathfrak{n}}$, which we shall identify. For any $\mathbf{a}, \mathbf{b} \in \mathbb{Z}_{\mathfrak{n}}^{\jmath \imath}$, let $\dot{\mathbf{K}}_{\mathbf{b}}^{\jmath \imath}$ denote the subspace of $\dot{\mathbf{K}}_{\mathfrak{n}}^{\jmath \imath}$ spanned by the standard basis element $[A]$ such that $\operatorname{ro}(A)=\mathbf{b}$ and $\operatorname{co}(A)=\mathbf{a}$. For any $\mathbf{b}, \mathbf{a}, \mathbf{b}^{\prime}, \mathbf{a}^{\prime} \in \mathbb{Z}_{\mathfrak{n}}^{\jmath \imath}$ and $\mathbf{b}^{\prime \prime}, \mathbf{a}^{\prime \prime} \in \mathbb{Z}_{\mathfrak{n}}^{\jmath \imath, a}$ such that $\left(\mathbf{b}^{\prime}, \mathbf{b}^{\prime \prime}\right) \models \mathbf{b}$ and $\left(\mathbf{a}^{\prime}, \mathbf{a}^{\prime \prime}\right) \models \mathbf{a}$, we define a linear map

$$
\Delta_{\mathbf{b}^{\prime}, \mathbf{a}^{\prime}, \mathbf{b}^{\prime \prime}, \mathbf{a}^{\prime \prime}}^{\jmath}:{ }_{\mathbf{b}} \dot{\mathbf{K}}_{\mathbf{a}}^{\jmath \imath} \longrightarrow{ }_{\mathbf{b}^{\prime}} \dot{\mathbf{K}}_{\mathbf{a}^{\prime}}^{\jmath \imath} \otimes{ }_{\mathbf{b}^{\prime \prime}} \dot{\mathbf{K}}_{\mathbf{a}^{\prime \prime}}
$$

by

$$
\Delta_{\mathbf{b}^{\prime}, \mathbf{a}^{\prime}, \mathbf{b}^{\prime \prime}, \mathbf{a}^{\prime \prime}}^{\jmath}([A])=\sum_{i, j} C_{i, j}(v, 1)\left[A_{i}^{\prime}\right] \otimes{ }^{\mathfrak{a}}\left[A_{j}^{\prime \prime}\right],
$$

where $\mathbf{b}^{\prime \prime} \dot{\mathbf{K}}_{\mathbf{a}^{\prime \prime}}$ is a component of $\dot{\mathbf{K}}_{\mathfrak{n}}, C_{i, j}\left(v, v^{\prime}\right), A_{i}^{\prime}$ and $A_{j}^{\prime \prime}$ are given in Proposition 10.2.6, We shall call the collection

$$
\dot{\Delta}^{\jmath}=\left(\Delta_{\mathbf{b}^{\prime}, \mathbf{a}^{\prime}, \mathbf{b}^{\prime \prime}, \mathbf{a}^{\prime \prime}}^{\imath}\right)_{\mathbf{b}^{\prime}, \mathbf{a}^{\prime} \in \mathbb{Z}_{\mathfrak{n}}^{\jmath \imath}, \mathbf{b}^{\prime \prime}, \mathbf{a}^{\prime \prime} \in \mathbb{Z}_{\mathfrak{n}}}
$$

the comultiplication of $\dot{\mathbf{K}}_{\mathfrak{n}}^{\jmath}$. Let $g_{A, B}^{C}$ and $h_{C}^{C^{\prime}, C^{\prime \prime}}$ be the structure constants of $\dot{\mathbf{K}}_{\mathfrak{n}}^{\jmath}$ of the multiplication and comultiplication, respectively, with respect to the standard bases. We have the following $\urcorner$-counterparts of the commutative diagrams (9.6.2) and (9.6.4) for the comultiplication $\dot{\Delta}^{\mathfrak{c}}$. 
Proposition 10.3.7. (1) The $\dot{\Delta}^{n}$ is an algebra homomorphism in the following sense: for all $A, B, C^{\prime} \in \widetilde{\Xi}_{\mathfrak{n}}^{\jmath n}, C^{\prime \prime} \in \widetilde{\Theta}_{\mathfrak{n}}$ one has

$$
\sum_{C \in \widetilde{\Xi}_{\mathfrak{n}}^{\jmath n}} g_{A, B}^{C} h_{C}^{C^{\prime}, C^{\prime \prime}}=\sum_{A^{\prime}, B^{\prime} \in \widetilde{\Xi}_{\mathfrak{n}}^{\jmath 2}, A^{\prime \prime}, B^{\prime \prime} \in \widetilde{\Theta}_{\mathfrak{n}}} h_{A}^{A^{\prime}, A^{\prime \prime}} h_{B}^{B^{\prime}, B^{\prime \prime}} g_{A^{\prime}, B^{\prime}}^{C^{\prime}} g_{A^{\prime \prime}, B^{\prime \prime}}^{C^{\prime \prime}}
$$

(2) The $\dot{\Delta}^{\jmath}$ is coassociative in the following sense: for all $A, A^{\prime} \in \widetilde{\Xi}_{\mathfrak{n}}^{\jmath \imath}, A^{\prime \prime}, A^{\prime \prime \prime} \in \widetilde{\Theta}_{\mathfrak{n}}$, one has

$$
\sum_{C \in \widetilde{\Xi}_{\mathfrak{n}}^{\jmath \imath}} h_{A}^{C, A^{\prime \prime \prime}} h_{C}^{A^{\prime}, A^{\prime \prime}}=\sum_{B \in \widetilde{\Theta}_{\mathfrak{n}}} h_{A}^{A^{\prime}, B}{ }^{\mathfrak{a}} h_{B}^{A^{\prime \prime}, A^{\prime \prime \prime}}
$$

Recall from Remark 9.5 .2 that $\dot{\mathbf{K}}_{\mathfrak{n}}$ is isomorphic to an idempotented quantum $\widehat{\mathfrak{g} l_{\mathfrak{n}}}$.

Proposition 10.3.8. The pair $\left(\dot{\mathbf{K}}_{\mathfrak{n}}, \dot{\mathbf{K}}_{\mathfrak{n}}^{\jmath n}\right)$ forms a quantum symmetric pair.

\subsection{Stabilization Algebra of TyPE $\vartheta$}

Recall the subalgebra $\mathbf{S}_{\mathfrak{n}, d}^{\imath \jmath}$ of $\mathbf{S}_{n, d}^{\mathfrak{c}}$ from (8.1.2). In analogue with the operator dlt ${ }_{r+1}$, we can define the operator $\mathrm{dlt}_{0}$. For each $\imath$-tridiagonal matrix $A \in \Xi_{\mathfrak{n}, d}^{\imath \jmath}$ (cf. (8.1.1)) such that $\operatorname{dlt}_{0}(A)-\sum_{1 \leqslant i \leqslant \mathfrak{n}} \alpha_{i} E_{\theta, \mathfrak{n}}^{i, i+1}$ is diagonal, we introduce the following element in $\mathbf{S}_{\mathfrak{n}, d}^{\imath \jmath}$ :

$$
\ddot{\mathbf{f}}_{A ; d}^{\jmath \jmath}=\mathbf{f}_{\mathfrak{n}}^{\left(\alpha_{\mathfrak{n}}\right)} * \mathbf{f}_{\mathfrak{n}-1}^{\left(\alpha_{\mathfrak{n}-1}\right)} * \cdots * \mathbf{f}_{1}^{\left(\alpha_{1}\right)} * \mathbf{f}_{0}^{\left(\alpha_{\mathfrak{n}}\right)} 1_{\operatorname{co}(A)} \in \mathbf{S}_{\mathfrak{n}, d}^{\imath \jmath} .
$$

Now repeat the process of the $\jmath$-version. We obtain an associative algebra $\dot{\mathbf{K}}_{\mathfrak{n}}^{\imath}$ with a basis $[A]$ parametrized by the matrices $A$ in $\widetilde{\Xi}_{\mathfrak{n}}^{\jmath}$, (which is defined exactly the same as $\widetilde{\Xi}_{\mathfrak{n}}^{\jmath}$, with the roles of $r+1$ and 0 switched). Moreover, to each matrix $A$ in $\widetilde{\Xi}_{\mathfrak{n}, d}^{\imath \jmath}$, we can define elements $\mathbf{f}_{A}^{\imath \jmath}, m_{A}^{\imath \jmath}$ and $\{A\}$ in $\dot{\mathbf{K}}_{\mathfrak{n}}^{\imath \jmath}$, similar to those elements indexed by $\jmath$ in $\dot{\mathbf{K}}_{\mathfrak{n}}^{\jmath}$, now starting with (10.4.1). Then all the main results for $\dot{\mathbf{K}}_{\mathfrak{n}}^{\jmath}$ admit counterparts for the algebra $\dot{\mathbf{K}}_{\mathfrak{n}}^{\imath \jmath}$.

Theorem 10.4.1. (1) The algebra $\dot{\mathbf{K}}_{\mathfrak{n}}^{\imath \jmath}$ admits a standard basis $\left\{[A] \mid A \in \widetilde{\Xi}_{\mathfrak{n}}^{\imath \jmath}\right\}$, a semimonomial basis $\left\{m_{A}^{\imath \jmath} \mid A \in \widetilde{\Xi}_{\mathfrak{n}}^{\imath \jmath}\right\}$, a monomial basis $\left\{\mathbf{f}_{A}^{\imath \jmath} \mid A \in \widetilde{\Xi}_{\mathfrak{n}}^{\imath \jmath}\right\}$, and a canonical basis $\left\{\{A\} \mid A \in \widetilde{\Xi}_{\mathfrak{n}}^{\imath \jmath}\right\}$.

(2) The assignment $[A] \mapsto[A]_{d}$, for all $A \in \widetilde{\Xi}_{\mathfrak{n}}^{\imath \jmath}$, defines a surjective algebra homomorphism $\Psi_{\mathfrak{n}, d}^{\imath \jmath}: \dot{\mathbf{K}}_{\mathfrak{n}}^{\imath \jmath} \rightarrow \mathbf{S}_{\mathfrak{n}, d}^{\imath \jmath}$ such that $\Psi_{\mathfrak{n}, d}^{\imath \jmath}(\{A\})=\{A\}_{d}$ if $A \in \Xi_{\mathfrak{n}, d}^{\imath \jmath}$ and zero otherwise.

(3) $\dot{\mathbf{K}}_{\mathfrak{n}}^{\imath \jmath}$ is a subquotient of $\dot{\mathbf{K}}_{n}^{\mathfrak{c}}$ with compatible stably canonical bases.

(4) The pair $\left(\dot{\mathbf{K}}_{\mathfrak{n}}, \dot{\mathbf{K}}_{\mathfrak{n}}^{\imath \jmath}\right)$ forms an idempotented quantum symmetric pair.

\subsection{StabilizATION ALGEBRA OF TYPE $\imath$}

Recall the subalgebra $\mathbf{S}_{\eta, d}^{\imath \imath}$ of $\mathbf{S}_{n, d}^{\mathfrak{c}}$ from (8.4.2). For each $\imath$-tridiagonal matrix $A \in \Xi_{\eta, d}^{\imath \imath}$ (cf. (8.1.1) ) such that the matrix $\operatorname{dlt}_{0, r}(A)-\sum_{1 \leqslant i \leqslant \eta} \alpha_{i} E_{\theta, \eta}^{i, i+1}$ is diagonal, we introduce the following element $\mathbf{f}_{A ; d}^{\imath u}$ in $\mathbf{S}_{\eta, d}^{\imath \imath}$ :

$$
\mathbf{f}_{A ; d}^{\imath \imath}=\mathbf{f}_{\eta+1}^{\left(\alpha_{\eta}\right)} * \mathbf{f}_{r}^{\left(\alpha_{r-1}\right)} *\left(\mathbf{f}_{\eta}^{\left(\alpha_{\eta-1}\right)} * \cdots * \mathbf{f}_{r+1}^{\left(\alpha_{r}\right)}\right) *\left(\mathbf{f}_{r-1}^{\left(\alpha_{r-1}\right)} * \cdots * \mathbf{f}_{0}^{\left(\alpha_{0}\right)}\right) * 1_{\operatorname{co}(A)} \in \mathbf{S}_{\eta, d}^{\imath \imath}
$$

We collect the main results of $\dot{\mathbf{K}}_{\eta}^{\imath}$ in the following. The proofs are very similar to the previous cases, and so we shall skip them to avoid redundancy. 
Theorem 10.5.1. (1) The algebra $\dot{\mathbf{K}}_{\eta}^{\imath \imath}$ admits a standard basis $\left\{[A] \mid A \in \widetilde{\Xi}_{\eta}^{\imath \imath}\right\}$, a semimonomial basis $\left\{m_{A}^{\imath \imath} \mid A \in \widetilde{\Xi}_{\eta}^{\imath \imath}\right\}$, a monomial basis $\left\{\mathbf{f}_{A}^{\imath \imath} \mid A \in \widetilde{\Xi}_{\eta}^{\imath \imath}\right\}$, and a canonical basis $\left\{\{A\} \mid A \in \widetilde{\Xi}_{\eta}^{n}\right\}$.

(2) The assignment $[A] \mapsto[A]_{d}$, for all $A \in \widetilde{\Xi}_{\eta}^{\imath 2}$, defines a surjective algebra homomorphism $\Psi_{\eta, d}^{\imath \imath}: \dot{\mathbf{K}}_{\eta}^{\imath \imath} \rightarrow \mathbf{S}_{\eta, d}^{\imath \imath}$ such that $\Psi_{\eta, d}^{\imath \imath}(\{A\})=\{A\}_{d}$ if $A \in \Xi_{\eta, d}^{\imath \imath}$ and zero otherwise.

(3) $\dot{\mathbf{K}}_{\eta}^{\imath \imath}$ is a subquotient of $\dot{\mathbf{K}}_{\mathfrak{n}}^{\jmath n}$ and $\dot{\mathbf{K}}_{\mathfrak{n}}^{\imath \jmath}$, with compatible stably canonical bases.

(4) The pair $\left(\dot{\mathbf{K}}_{\eta}, \dot{\mathbf{K}}_{\eta}^{\imath \imath}\right)$ is an idempotented quantum symmetric pair.

Let us summarize the interrelations among different family of Schur algebras, as well as the interrelations among different family of stabilization algebras of types $\jmath \jmath, \jmath, \imath \jmath, \imath$.

Recall $\breve{n}=n+2, n=\mathfrak{n}+1$, and $\mathfrak{n}=\eta+1$, where $n$ is even. On the Schur algebra level, we have the following commutative diagram for natural inclusions of Schur algebras:

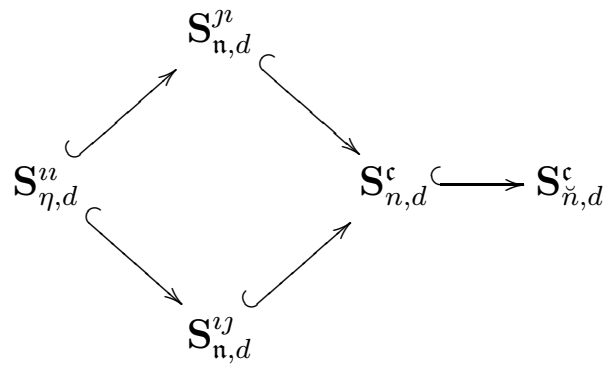

On the stabilization algebra level, we have the following diagram of subquotients:

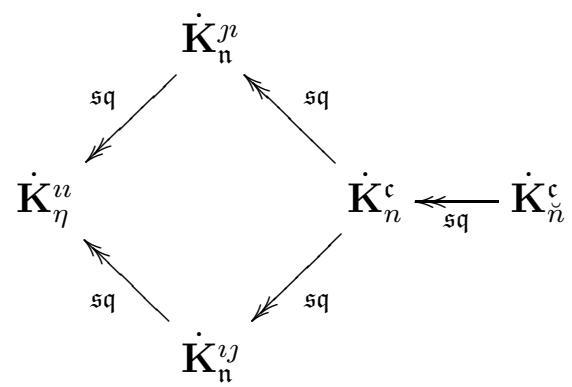

where the notation $\mathbf{K}_{1} \stackrel{\text { sq }}{\rightarrow} \mathbf{K}_{2}$ stands for the statement that $\mathbf{K}_{2}$ is a subquotient of $\mathbf{K}_{1}$. Remarkably, all the subquotients between various pairs of algebras preserve the stably canonical bases.

Remark 10.5.2. One can show that the Schur algebras $\mathbf{S}_{\mathfrak{n}, d}^{\jmath \imath}$ and $\mathbf{S}_{\mathfrak{n}, d}^{\imath \jmath}$ are isomorphic with compatible standard and canonical bases. This isomorphism can be further lifted to the stabilization level. The proofs of these isomorphisms is given in [FL17]. 


\section{APPENDIX A}

\section{Constructions in finite type $C$}

We shall present more details on results in finite type $C$ which was only sketched in [BKLW14]. In addition, we will present details on comultiplications and transfer maps in finite type $C$, adapting the finite type $B$ formulation in [FL15]. This will serve as a helpful preparation for formulation and computations in affine type $C$ which are presented in the main text.

\section{A.1. Multiplication Formulas}

Recall that $\mathfrak{n}=2 r+1$. We fix a non-degenerate skew-symmetric bilinear form $Q$ : $\mathbb{F}_{q}^{2 d} \times \mathbb{F}_{q}^{2 d} \rightarrow \mathbb{F}_{q}$. Let $\operatorname{Sp}(2 d)$ be the symplectic subgroup of $G L(2 d)$ which consists of all elements $g$ such that $Q\left(g u, g u^{\prime}\right)=Q\left(u, u^{\prime}\right), \forall u, u^{\prime} \in \mathbb{F}_{q}^{2 d}$. Consider the following sets

$$
\begin{aligned}
& X_{\mathfrak{c}}=\left\{0=L_{0} \subset L_{1} \subset \ldots \subset L_{\mathfrak{n}}=\mathbb{F}_{q}^{2 d} \mid L_{\mathfrak{n}-i}=L_{i}^{\perp}\right\}, \\
& Y_{\mathfrak{c}}=\left\{0=L_{0} \stackrel{1}{\subset} L_{1} \stackrel{1}{\subset} \ldots \stackrel{1}{\subset} L_{2 d}=\mathbb{F}_{q}^{2 d} \mid L_{2 d-i}=L_{i}^{\perp}\right\}, \\
& { }^{\mathfrak{c}} \Xi=\left\{A=\left(a_{i j}\right) \in \operatorname{Mat}_{\mathfrak{n} \times \mathfrak{n}}(\mathbb{N}) \mid \sum_{i, j \in[1, \mathfrak{n}]} a_{i j}=2 d, a_{i j}=a_{\mathfrak{n}+1-i, \mathfrak{n}+1-j}, \forall i, j \in[1, \mathfrak{n}]\right\}, \\
& { }^{\mathfrak{c}} \Pi=\left\{B=\left(b_{i j}\right) \in \operatorname{Mat}_{\mathfrak{n} \times 2 d}(\mathbb{N}) \mid \sum_{i \in[1, \mathfrak{n}]} b_{i j}=1, b_{i j}=b_{\mathfrak{n}+1-i, 2 d+1-j}, \forall i \in[1, \mathfrak{n}], j \in[1,2 d]\right\}, \\
& { }^{\mathfrak{c}} \Sigma=\left\{\sigma=\left(\sigma_{i j}\right) \in \operatorname{Mat}_{2 d \times 2 d}(\mathbb{N}) \mid \sum_{i \in[1,2 d]} \sigma_{i j}=1=\sum_{j \in[1,2 d]} \sigma_{i j},\right. \\
& \left.\sigma_{i j}=\sigma_{2 d+1-i, 2 d+1-j}, \forall i, j \in[1,2 d]\right\} .
\end{aligned}
$$

The notation $\stackrel{1}{\subset}$ above denotes inclusion of codimension 1 as before. The action of $\operatorname{Sp}(2 d)$ on $\mathbb{F}_{q}^{2 d}$ induces a well-defined action of $\operatorname{Sp}(2 d)$ on $X_{\mathfrak{c}}$ and $Y_{\mathfrak{c}}$. Let $\operatorname{Sp}(2 d)$ act diagonally on $X_{\mathfrak{c}} \times X_{\mathfrak{c}}$ and $Y_{\mathfrak{c}} \times Y_{\mathfrak{c}}$.

Lemma A.1.1. [BKLW14, Lemma 6.5] There are natural bijections $\operatorname{Sp}(2 d) \backslash X_{\mathfrak{c}} \times X_{\mathfrak{c}} \longleftrightarrow{ }^{\mathfrak{c}} \Xi$, $\operatorname{Sp}(2 d) \backslash X_{\mathfrak{c}} \times Y_{\mathfrak{c}} \longleftrightarrow{ }^{\mathfrak{c}} \Pi$, and $\operatorname{Sp}(2 d) \backslash Y_{\mathfrak{c}} \times Y_{\mathfrak{c}} \longleftrightarrow{ }^{\mathfrak{c}} \Sigma$.

Let ${ }^{\mathrm{c}} \mathrm{S}_{d}^{\jmath}=\mathcal{A}_{\mathrm{Sp}(2 d)}\left(X_{\mathfrak{c}} \times X_{\mathfrak{c}}\right)$ be the algebra of $\operatorname{Sp}(2 d)$-invariant $\mathcal{A}$-valued functions on $X_{\mathfrak{c}} \times X_{\mathfrak{c}}$, where $\mathcal{A}=\mathbb{Z}\left[v, v^{-1}\right]$ and the multiplication is given by a convolution product.

The most typical phenomenon of type $C$ already shows up when $\mathfrak{n}=5$, and so let us consider this case in detail. Let $V_{k}$ be a $2 d$-dimensional vector space over $k=\mathbb{F}_{q}$ equipped with a non-degenerate symplectic form. Let $\left(L_{i} \mid 0 \leqslant i \leqslant 5\right)$ be a flag of vector subspaces in $V_{k}$ such that $L_{i}^{\perp}=L_{5-i}$ for $i \in[0,5]$. Consider the set

$$
Z_{i}=\left\{U \subseteq V_{k} \mid \operatorname{dim}_{k} U=1, U \subseteq L_{i}, U \nsubseteq L_{i-1}\right\}, \quad \forall i \in[1,4] .
$$

The following lemma is an analogue of [FL14, Lemma 3.1.3] with an easier proof. 
Lemma A.1.2. For $\mathfrak{n}=5$, we have $\# Z_{3}=q^{\operatorname{dim} L_{2}} \frac{q^{\operatorname{dim} L_{3} / L_{2}-1}}{q-1}$ and $\# Z_{4}=q^{\operatorname{dim} L_{3}} \frac{q^{\operatorname{dim} L_{4} / L_{3}-1}}{q-1}$.

Proof. Because all lines in $V_{k}$ are isotropic, we have

$$
\# Z_{3}=\frac{q^{\operatorname{dim} L_{3}}-1}{q-1}-\frac{q^{\operatorname{dim} L_{2}}-1}{q-1}=q^{\operatorname{dim} L_{2}} \frac{q^{\operatorname{dim} L_{3} / L_{2}}-1}{q-1} .
$$

The counting for $Z_{4}$ is the same.

We have the following multiplication formula in finite type $C$. Let $E_{i, j}$ for all $1 \leqslant i, j \leqslant \mathfrak{n}$, the standard basis of the space of $\mathfrak{n}$ by $\mathfrak{n}$ matrices. We set $E_{i, j}^{\theta}=E_{i, j}+E_{\mathfrak{n}-i, \mathfrak{n}-j}$ for all $1 \leqslant i, j \leqslant \mathfrak{n}$.

Proposition A.1.3. Suppose that $h \in[1, r]$ and $R \in \mathbb{N}$.

(1) For $A, B \in{ }^{\mathfrak{c}} \Xi$ such that $\operatorname{ro}(A)=\operatorname{co}(B)$ and $B-R E_{h, h+1}^{\theta}$ is diagonal, we have

$$
e_{B} * e_{A}=\sum_{t} v^{2 \sum_{j>u} a_{h j} t_{u}} \prod_{u=1}^{\mathfrak{n}}\left[\begin{array}{c}
a_{h u}+t_{u} \\
t_{u}
\end{array}\right] e_{A+\sum_{u=1}^{\mathfrak{n}} t_{u}\left(E_{h u}^{\theta}-E_{h+1, u}^{\theta}\right)}
$$

where $t=\left(t_{u}\right) \in \mathbb{N}^{\mathfrak{n}}$ such that $\sum_{u=1}^{\mathfrak{n}} t_{u}=R$ and $\begin{cases}t_{u} \leqslant a_{h+1, u}, & \text { if } h<r \\ t_{u}+t_{\mathfrak{n}+1-u} \leqslant a_{h+1, u}, & \text { if } h=r .\end{cases}$

(2) For $A, C \in{ }^{\mathfrak{c}} \Xi$ such that $\operatorname{ro}(A)=\operatorname{co}(C)$ and $C-R E_{h+1, h}^{\theta}$ is diagonal, we have

$$
\begin{aligned}
e_{C} * e_{A} & =\sum_{t} v^{2 \sum_{j<u} a_{h+1, j} t_{u}} \prod_{u=1}^{\mathfrak{n}}\left[\begin{array}{c}
a_{h+1, u}+t_{u} \\
t_{u}
\end{array}\right] e_{A-\sum_{u=1}^{\mathfrak{n}} t_{u}\left(E_{h u}^{\theta}-E_{h+1, u}^{\theta}\right)}, \text { if } h<r ; \\
e_{C} * e_{A} & =\sum_{t} v^{2 \sum_{j<u} a_{r+1, j} t_{u}+2 \sum_{\mathfrak{n}+1-j<u<j} t_{u} t_{j}+\sum_{u>r+1} t_{u}\left(t_{u}+1\right)} \prod_{u<r+1}\left[\begin{array}{c}
a_{r+1, u}+t_{u} \\
t_{u}
\end{array}\right] \\
& \cdot \prod_{u>r+1}\left[\begin{array}{c}
a_{r+1, u}+t_{u}+t_{\mathfrak{n}+1-u} \\
t_{u}
\end{array}\right] \prod_{i=1}^{t_{r+1}} \frac{\left[a_{r+1, r+1}+2 i\right]}{[i]} e_{A-\sum_{u=1}^{\mathfrak{n}} t_{u}\left(E_{r u}^{\theta}+E_{r+1, u}^{\theta}\right)} \text { if } h=r,
\end{aligned}
$$

where $t=\left(t_{u}\right) \in \mathbb{N}^{\mathfrak{n}}$ such that $\sum_{u=1}^{\mathfrak{n}} t_{u}=R$ and $t_{u} \leqslant a_{h u}$.

Proof. We only give a sketch as it is similar to [BKLW14, FL14. First the proposition is proved for $R=1$ with the help of Lemma A.1.2 (which takes care of a genuine type $C$ counting). Then a similar argument using induction as in [BKLW14, Proposition 3.3] or [FL14, Corollary 4.3.4] proves the general case.

For $A=\left(a_{i j}\right) \in{ }^{\mathfrak{c}} \Xi$, we set

$$
d(A)=\operatorname{dim} \mathcal{O}_{A} \quad \text { and } \quad d_{A}=d(A)-d(B),
$$

where $B=\left(b_{i j}\right)$ is the diagonal matrix such that $b_{i i}=\sum_{k} a_{i k}$.

Lemma A.1.4. For any $A=\left(a_{i j}\right) \in{ }^{\mathfrak{c}} \Xi$, we have

$$
d_{A}=\frac{1}{2}\left(\sum_{i \geqslant k, j<l} a_{i j} a_{k l}+\sum_{i \geqslant r+1>j} a_{i j}\right) .
$$

Proof. The proof is similar to the proof of [BKLW14, Lemma 3.5] or [FL14, Lemma 4.5.1]. See also the proof of Lemma 4.1.1. 
We set

$$
[A]=v^{-d_{A}} e_{A} . \quad \forall A \in{ }^{\mathfrak{c}} \Xi .
$$

It is clear that $\left\{[A] \mid A \in{ }^{\mathfrak{c}} \Xi\right\}$ form an $\mathcal{A}$-basis of ${ }^{\mathfrak{c}} \mathbf{S}_{d}^{\jmath}$, which is called a standard basis.

By a direct calculation using (A.1.4), we have the following reformulation of Proposition A.1.3 in terms of $[A]$.

Proposition A.1.5. Suppose that $A, B, C \in{ }^{\mathfrak{c}} \Xi, h \in[1, r]$ and $R \in \mathbb{N}$.

(1) If $\operatorname{ro}(A)=\operatorname{co}(B)$ and $B-R E_{h, h+1}^{\theta}$ is diagonal, then we have

$$
[B] *[A]=\sum_{t} v^{\beta(t)} \prod_{u=1}^{\mathfrak{n}} \overline{\left[\begin{array}{c}
a_{h u}+t_{u} \\
t_{u}
\end{array}\right]}\left[A+\sum_{u=1}^{\mathfrak{n}} t_{u}\left(E_{h u}^{\theta}-E_{h+1, u}^{\theta}\right)\right],
$$

where the sum over $t$ is as in Proposition A.1.3(1) and

$$
\beta(t)=\sum_{u \leqslant j} a_{h j} t_{u}-\sum_{u<j} a_{h+1, j} t_{u}+\sum_{u<j} t_{u} t_{j}+\delta_{h n}\left(\sum_{\substack{u<j \\ u+j<\mathfrak{n}+1}} t_{u} t_{j}+\sum_{u<r+1} \frac{t_{u}\left(t_{u}-1\right)}{2}\right) .
$$

(2) Assume that $\operatorname{ro}(A)=\operatorname{co}(C)$ and $C-R E_{h+1, h}^{\theta}$ is diagonal. Then for $h<r$ we have

$$
[C] *[A]=\sum_{t} v^{\beta^{\prime}(t)} \prod_{u=1}^{\mathfrak{n}} \overline{\left[\begin{array}{c}
a_{h+1, u}+t_{u} \\
t_{u}
\end{array}\right]}\left[A-\sum_{u=1}^{\mathfrak{n}} t_{u}\left(E_{h u}^{\theta}-E_{h+1, u}^{\theta}\right)\right],
$$

where the sum over $t$ is as in Proposition A.1.3(2) and

$$
\beta^{\prime}(t)=\sum_{u \geqslant j} a_{h+1, j} t_{u}-\sum_{u>j} a_{h j} t_{u}+\sum_{u>j} t_{u} t_{j}
$$

For $h=r$, we have

$$
\begin{aligned}
{[C] *[A]=} & \sum_{t} v^{\gamma(t)} \prod_{u>r+1} \overline{\left[\begin{array}{c}
a_{r+1, u}+t_{u} \\
t_{u}
\end{array}\right]} \prod_{u<r+1} \overline{\left[\begin{array}{c}
a_{r+1, u}+t_{u}+t_{\mathfrak{n}+1-u} \\
t_{u}
\end{array}\right]} \\
& \cdot \prod_{i=1}^{t_{r+1}} \frac{\overline{\left[a_{r+1, r+1}+2 i\right]}}{\overline{[i]}}\left[A-\sum_{u=1}^{\mathfrak{n}} t_{u}\left(E_{r u}^{\theta}+E_{r+1, u}^{\theta}\right)\right],
\end{aligned}
$$

where $\gamma(t)=\sum_{u \leqslant j} a_{r+1, j} t_{u}-\sum_{u>j} a_{h j} t_{u}+\sum_{\mathfrak{n}+1-j \leqslant u<j} t_{u} t_{j}-\sum_{u<r+1} \frac{t_{u}^{2}}{2}+\frac{R^{2}}{2}+\sum_{u \geqslant r+1} \frac{t_{u}}{2}$.

Let

$$
\begin{aligned}
\widetilde{\Xi}_{\mathfrak{c}}=\left\{A=\left(a_{i j}\right) \in \operatorname{Mat}_{\mathfrak{n} \times \mathfrak{n}}(\mathbb{Z}) \mid a_{i j} \geqslant 0 \text { if } i \neq j,\right. \\
\left.a_{i j}=a_{\mathfrak{n}+1-i, \mathfrak{n}+1-j}, \quad \forall i, j, \text { and } a_{r+1, r+1} \in 2 \mathbb{Z}\right\} .
\end{aligned}
$$

Denoted by ${ }^{\mathfrak{c}} \mathbf{K}^{\jmath}$ the free $\mathcal{A}$-module spanned by $\left\{[A] \mid A \in \widetilde{\Xi}_{\mathfrak{c}}\right\}$. For any matrix $A$, we set

$$
{ }_{2 p} A=A+2 p I \text {. }
$$

Here $I=\sum_{1 \leqslant i \leqslant \mathfrak{n}} E_{i i}$. By a similar argument as that for Proposition 4.2 in [BLM90], we have the following proposition. 
Proposition A.1.6. Suppose that $A_{1}, \ldots, A_{s} \in \widetilde{\Xi}_{\mathfrak{c}}(s \geqslant 2)$ satisfy that $\operatorname{co}\left(A_{i}\right)=\operatorname{ro}\left(A_{i+1}\right)$ for all $i$. Then there exist $Z_{1}, \ldots, Z_{m} \in \widetilde{\Xi}_{\mathfrak{c}},{ }^{\mathfrak{c}} G_{i}\left(v, v^{\prime}\right) \in \mathbb{Q}(v)\left[v^{\prime}\right]$ such that

$$
\left[{ }_{2 p} A_{1}\right] *\left[{ }_{2 p} A_{2}\right] * \cdots *\left[{ }_{2 p} A_{s}\right]=\sum_{i=1}^{m}{ }^{\mathfrak{c}} G_{i}\left(v, v^{-2 p}\right)\left[{ }_{2 p} Z_{i}\right], \quad \text { for } p \gg 0 .
$$

By specialization at $v^{\prime}=1$, we have the following corollary.

Corollary A.1.7. Retain the assumption in Proposition A.1.6. There is a unique associative $\mathcal{A}$-algebra structure on ${ }^{\mathfrak{c}} \mathbf{K}^{\mathfrak{J}}$ given by

$$
\left[A_{1}\right] *\left[A_{2}\right] * \cdots *\left[A_{s}\right]=\sum_{i=1}^{m} G_{i}(v, 1)\left[Z_{i}\right] .
$$

\section{A.2. Isomorphisms BetWeEn TYPe C AND TYPE B}

Recall that $\mathbf{S}^{\jmath}=\mathbf{S}_{d}^{\jmath}$ is the convolution algebra on $\mathfrak{n}$-step type $B$ flags defined in [BKLW14], and it admits a standard basis $\left\{[A] \mid A \in{ }^{\mathfrak{b}} \Xi\right\}$, where $\left({ }^{\mathfrak{b}} \Xi\right.$ is denoted by $\Xi_{d}$ in loc. cit.)

$$
{ }^{\mathfrak{b}} \Xi=\left\{A=\left(a_{i j}\right) \in \operatorname{Mat}_{\mathfrak{n} \times \mathfrak{n}}(\mathbb{N}) \mid \sum_{i, j \in[1, \mathfrak{n}]} a_{i j}=2 d+1, a_{i j}=a_{\mathfrak{n}+1-i, \mathfrak{n}+1-j}, \forall i, j \in[1, \mathfrak{n}]\right\} .
$$

Clearly sending $A \mapsto A-E_{r+1, r+1}$ defines a bijection ${ }^{\mathfrak{b}} \Xi \stackrel{\sim}{\longrightarrow}{ }^{\mathfrak{c}} \Xi$. Let $\psi: \mathbf{S}_{d}^{\jmath} \rightarrow{ }^{\mathfrak{c}} \mathbf{S}_{d}^{\jmath}$ be the $\mathcal{A}$-linear map sending $[A] \mapsto\left[A-E_{r+1, r+1}\right]$ for all $A \in{ }^{\mathfrak{c}} \Xi$. It is clear that $\psi$ is an $\mathcal{A}$-linear isomorphism.

Proposition A.2.1. The map $\psi: \mathbf{S}_{d}^{\jmath} \longrightarrow{ }^{\mathrm{c}} \mathbf{S}_{d}^{\jmath}$ is an $\mathcal{A}$-algebra isomorphism.

Proof. Since the structure of the two algebras are completely determined by the multiplication formulas in Proposition A.1.5 and [BKLW14, Proposition 3.7], we only need to see if they match under the correspondence $[A] \mapsto\left[A-E_{r+1, r+1}\right]$, which can be checked directly.

Let $\tilde{\psi}: \mathbf{K}^{\jmath} \rightarrow{ }^{\mathfrak{c}} \mathbf{K}^{\jmath}$ be the $\mathcal{A}$-linear map sending $[A] \mapsto\left[A-E_{r+1, r+1}\right]$ for all $A \in \widetilde{\Xi}_{\mathfrak{c}}$, where $\mathbf{K}^{\jmath}$ is the algebra defined in [BKLW14, Section 4], a finite type $B$ counterpart of ${ }^{\mathfrak{c}} \mathbf{K}^{\jmath}$. The algebra isomorphisms $\psi: \mathbf{S}_{d}^{\jmath} \longrightarrow{ }^{\mathfrak{c}} \mathbf{S}_{d}^{\jmath}$ (for varies $d$ ) and the stabilization procedure (Proposition A.1.6 and Corollary A.1.7) which defines the algebra ${ }^{\mathfrak{c}} \mathbf{K}^{\jmath}$ (and similar for $\mathbf{K}^{\jmath}$ ) lead readily to the following identification.

Proposition A.2.2. The map $\tilde{\psi}: \mathbf{K}^{\jmath} \longrightarrow{ }^{\mathfrak{c}} \mathbf{K}^{\jmath}$ is an $\mathcal{A}$-algebra isomorphism.

\section{A.3. The COMUltiplication}

We define $\mathbf{e}_{i}, \mathbf{f}_{i}, \mathbf{h}_{a}^{ \pm 1} \in{ }^{\mathfrak{c}} \mathbf{S}_{d}^{\jmath}$, for $i \in[1, r]$ and $a \in[1, r+1]$, as follows: for all $L, L^{\prime} \in X_{\mathfrak{c}}$,

$$
\begin{aligned}
\mathbf{e}_{i}\left(L, L^{\prime}\right) & = \begin{cases}v^{-\left|L_{i+1}^{\prime} / L_{i}^{\prime}\right|-\delta_{i, r},} & \text { if } L_{i} \stackrel{1}{\subset} L_{i}^{\prime}, L_{j}=L_{j}^{\prime}, \forall j \in[1, r] \backslash\{i\} ; \\
0, & \text { otherwise. }\end{cases} \\
\mathbf{f}_{i}\left(L, L^{\prime}\right) & = \begin{cases}v^{-\left|L_{i}^{\prime} / L_{i-1}^{\prime}\right|}, & \text { if } L_{i} \stackrel{1}{\supset} L_{i}^{\prime}, L_{j}=L_{j}^{\prime}, \forall j \in[1, r] \backslash\{i\} ; \\
0, & \text { otherwise. }\end{cases} \\
\mathbf{h}_{a}^{ \pm 1}\left(L, L^{\prime}\right) & =v^{ \pm\left(\left|L_{a}^{\prime} / L_{a-1}^{\prime}\right|+\delta_{a, r+1}\right)} \delta_{L, L^{\prime}} .
\end{aligned}
$$


Also set $\mathbf{k}_{i}=\mathbf{h}_{i+1} \mathbf{h}_{i}^{-1}$ in $\mathbf{S}_{d}^{J}$. Note that our $\mathbf{h}_{a}$ corresponds to $\mathbf{d}_{a}^{-1}$ in [BKLW14, (3.3)], and the definitions of $\mathbf{e}_{i}, \mathbf{f}_{i}, \mathbf{h}_{a}$ above for finite type $C$ formally coincide with those for finite type $B$ [BKLW14, (3.1)-(3.3)] (except $\left.\mathbf{e}_{r}, \mathbf{f}_{r}, \mathbf{h}_{r+1}\right)$.

Proposition A.3.1. The isomorphism $\psi: \mathbf{S}_{d}^{\jmath} \longrightarrow{ }^{\mathfrak{c}} \mathbf{S}_{d}^{\jmath}$ sends $\mathbf{e}_{i}, \mathbf{f}_{i}, \mathbf{h}_{a}:=\mathbf{d}_{a}^{-1}$ for $i \in[1, r]$ and $a \in[1, r+1]$ in $\mathbf{S}_{d}^{j}$ to the elements in ${ }^{\mathrm{c}} \mathbf{S}_{d}^{j}$ in the same notations, respectively.

Proof. The element $\mathbf{e}_{i}$ on both sides is a sum of all standard matrices $A$ such that $A-E_{i+1, i}^{\theta}$ is diagonal. Hence we have the result for $\mathbf{e}_{i}$ by the definition of $\psi$. Similarly, one can prove the results for $\mathbf{f}_{i}$ and $\mathbf{h}_{a}$.

We shall denote by $\mathbf{S}_{d}$ the Schur algebra of finite type $A$ arising from $\mathfrak{n}$-step flags in an $d$-dimensional space. For any $i \in[1, \mathfrak{n}-1], a \in[1, \mathfrak{n}]$, we define the following elements in $\mathbf{S}_{d}$ :

$$
\begin{aligned}
\mathbf{E}_{i}\left(V, V^{\prime}\right) & = \begin{cases}v^{-\left|V_{i+1}^{\prime} / V_{i}^{\prime}\right|}, & \text { if } V_{i} \stackrel{1}{\subset} V_{i}^{\prime}, V_{j}=V_{j^{\prime}}, \forall j \neq i ; \\
0, & \text { otherwise. }\end{cases} \\
\mathbf{F}_{i}\left(V, V^{\prime}\right) & = \begin{cases}v^{-\left|V_{i}^{\prime} / V_{i-1}^{\prime}\right|}, & \text { if } V_{i} \stackrel{1}{\supset} V_{i}^{\prime}, V_{j}=V_{j^{\prime}}, \forall j \neq i ; \\
0, & \text { otherwise, }\end{cases} \\
\mathbf{H}_{a}^{ \pm 1}\left(V, V^{\prime}\right) & =v^{ \pm\left|V_{a} / V_{a-1}\right|} \delta_{V, V^{\prime}}, \quad \forall V, V^{\prime} \in X_{d} . \\
\mathbf{K}_{i}^{ \pm 1} & =\mathbf{H}_{i+1}^{ \pm 1} \mathbf{H}_{i}^{\mp 1} .
\end{aligned}
$$

In a completely analogous way to the definition $\widetilde{\Delta}^{\jmath}$ in [FL15, §3.2], for a composition $d=d^{\prime}+d^{\prime \prime}$, we have a comultiplication

$$
\widetilde{\Delta}^{\mathfrak{c}}: \mathbf{S}_{d}^{\mathfrak{c}} \longrightarrow \mathbf{S}_{d^{\prime}}^{\mathfrak{c}} \otimes \mathbf{S}_{d^{\prime \prime}}
$$

Then we have the following proposition, similar to [FL15, Proposition 3.2.4].

Proposition A.3.2. For any $i \in[1, r]$, we have

$$
\begin{aligned}
\widetilde{\Delta}^{\mathfrak{c}}\left(\mathbf{e}_{i}\right) & =\mathbf{e}_{i}^{\prime} \otimes \mathbf{H}_{i+1}^{\prime \prime} \mathbf{H}_{\mathfrak{n}-i}^{\prime \prime-1}+\mathbf{h}_{i+1}^{\prime-1} \otimes \mathbf{E}_{i}^{\prime \prime} \mathbf{H}_{\mathfrak{n}-i}^{\prime \prime-1}+\mathbf{h}_{i+1}^{\prime} \otimes \mathbf{F}_{\mathfrak{n}-i}^{\prime \prime} \mathbf{H}_{i+1}^{\prime \prime} . \\
\widetilde{\Delta}^{\mathfrak{c}}\left(\mathbf{f}_{i}\right) & =\mathbf{f}_{i}^{\prime} \otimes \mathbf{H}_{i}^{\prime \prime-1} \mathbf{H}_{\mathfrak{n}+1-i}^{\prime \prime}+\mathbf{h}_{i}^{\prime} \otimes \mathbf{F}_{i}^{\prime \prime} \mathbf{H}_{\mathfrak{n}+1-i}^{\prime \prime}+\mathbf{h}_{i}^{\prime-1} \otimes \mathbf{E}_{\mathfrak{n}-i}^{\prime \prime} \mathbf{H}_{i}^{\prime \prime-1} . \\
\widetilde{\Delta}^{\mathfrak{c}}\left(\mathbf{k}_{i}\right) & =\mathbf{k}_{i}^{\prime} \otimes \mathbf{K}_{i}^{\prime \prime} \mathbf{K}_{\mathfrak{n}-i}^{\prime \prime-1} .
\end{aligned}
$$

Proof. With the help of Lemma A.1.2, the proof of [FL15, Proposition 3.2.4] can be essentially repeated here.

By checking the image of algebra generators of $\mathbf{S}_{d}^{J}$, we have the following proposition.

Proposition A.3.3. The following diagram is commutative:

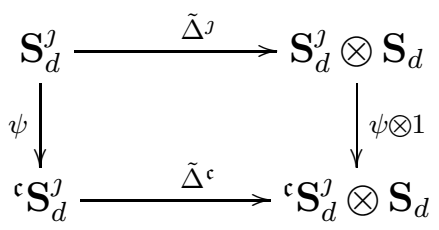

Following [FL15], we introduce the following notation

$$
\Lambda_{\mathfrak{n}, d}^{\jmath}=\left\{\mathbf{a}=\left(a_{i}\right) \in \mathbb{N}^{\mathfrak{n}} \mid \sum a_{i}=2 d+1, a_{i}=a_{\mathfrak{n}+1-i}\right\} .
$$


An isotropic flag $L$ of type $C$ defines a unique element $\alpha(L) \in \Lambda_{\mathfrak{n}, d}^{J}$ by

$$
\alpha(L)_{i}=\operatorname{dim} L_{i} / L_{i-1}+\delta_{i, r+1}, \forall i .
$$

Then we have the following partition:

$$
X_{\mathfrak{c}}=\bigsqcup_{\mathbf{a} \in \Lambda_{\mathfrak{n}, d}^{J}} X_{\mathfrak{c}}(\mathbf{a}), \quad X_{\mathfrak{c}}(\mathbf{a})=\{L \mid \alpha(L)=\mathbf{a}\} .
$$

For any $\mathbf{a}, \mathbf{b} \in \Lambda_{\mathfrak{n}, d}^{\jmath}$, let ${ }^{\mathfrak{c}} \mathbf{S}_{d}^{\jmath}(\mathbf{b}, \mathbf{a})$ be the subspace of ${ }^{\mathrm{c}} \mathbf{S}_{d}^{\jmath}$ spanned by all functions supported on $X_{\mathfrak{c}}(\mathbf{b}) \times X_{\mathfrak{c}}(\mathbf{a})$. Then we have

$$
{ }^{\mathfrak{c}} \mathbf{S}_{d}^{\mathcal{J}}=\oplus_{\mathbf{b}, \mathbf{a} \in \Lambda_{\mathfrak{n}, d}^{\mathcal{J}}} \mathbf{S}_{d}^{\mathcal{J}}(\mathbf{b}, \mathbf{a}) .
$$

We shall denote $\iota_{\mathbf{b}, \mathbf{a}}$ and $p_{\mathbf{b}, \mathbf{a}}$ the embedding of ${ }^{\mathrm{c}} \mathbf{S}_{d}^{\jmath}(\mathbf{b}, \mathbf{a})$ into ${ }^{\mathrm{c}} \mathbf{S}_{d}^{\jmath}$ and the projection of ${ }^{\mathrm{c}} \mathbf{S}_{d}^{\jmath}$ to ${ }^{\mathbf{c}} \mathbf{S}_{d}^{\jmath}(\mathbf{b}, \mathbf{a})$, respectively. By abuse of notations, the projection from $\mathbf{S}_{d}$ to $\mathbf{S}_{d}(\mathbf{b}, \mathbf{a})$ is still denoted by $p_{\mathbf{b}, \mathbf{a}}$. For any $\mathbf{b}, \mathbf{a}, \mathbf{b}^{\prime}, \mathbf{a}^{\prime}, \mathbf{b}^{\prime \prime}$ and $\mathbf{a}^{\prime \prime}$ satisfying that

$$
b_{i}=b_{i}^{\prime}+b_{i}^{\prime \prime}+b_{\mathfrak{n}+1-i}^{\prime \prime}, \quad \text { and } \quad a_{i}=a_{i}^{\prime}+a_{i}^{\prime \prime}+a_{\mathfrak{n}+1-i}^{\prime \prime}, \quad \forall i \in[1, \mathfrak{n}],
$$

we set $\tilde{\Delta}_{\mathbf{b}^{\prime}, \mathbf{a}^{\prime}, \mathbf{b}^{\prime \prime}, \mathbf{a}^{\prime \prime}}^{\mathfrak{c}}=\left(p_{\mathbf{b}^{\prime}, \mathbf{a}^{\prime}} \otimes p_{\mathbf{b}^{\prime \prime}, \mathbf{a}^{\prime \prime}}\right) \circ \tilde{\Delta}^{\mathfrak{c}} \circ \iota_{\mathbf{b}, \mathbf{a}}$. Let

$$
\Delta^{\mathfrak{c}}=\bigoplus_{\mathbf{b}, \mathbf{a}, \mathbf{b}^{\prime}, \mathbf{a}^{\prime}, \mathbf{b}^{\prime \prime}, \mathbf{a}^{\prime \prime}} \Delta_{\mathbf{b}^{\prime}, \mathbf{a}^{\prime}, \mathbf{b}^{\prime \prime}, \mathbf{a}^{\prime \prime}}^{\mathfrak{c}}
$$

where $\Delta_{\mathbf{b}^{\prime}, \mathbf{a}^{\prime}, \mathbf{b}^{\prime \prime}, \mathbf{a}^{\prime \prime}}^{\mathfrak{c}}=v^{\sum_{1 \leqslant i \leqslant j \leqslant n} b_{i}^{\prime} b_{j}^{\prime \prime}-a_{i}^{\prime} a_{j}^{\prime \prime}} v^{u\left(\mathbf{b}^{\prime \prime}, \mathbf{a}^{\prime \prime}\right)} \tilde{\Delta}_{\mathbf{b}^{\prime}, \mathbf{a}^{\prime}, \mathbf{b}^{\prime \prime}, \mathbf{a}^{\prime \prime}}^{\mathfrak{c}}$, and $u(\mathbf{b}, \mathbf{a})$ is the function defined in [FL15, (44)] in finite type $B$ setting. The definition of $\Delta^{\mathfrak{c}}$ is completely analogous to the definition of $\Delta_{\mathbf{v}}^{J}$ in [FL15, (45)]. The following proposition follows by comparing the definitions.

Proposition A.3.4. Given $d=d^{\prime}+d^{\prime \prime}$, we have the following commutative diagram:

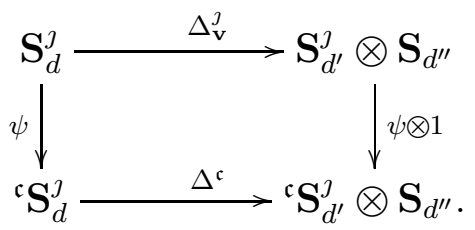

The transfer map

$$
\phi_{d, d-\mathfrak{n}}^{\mathfrak{c}}:{ }^{\mathfrak{c}} \mathrm{S}_{d} \longrightarrow{ }^{\mathfrak{c}} \mathbf{S}_{d-\mathfrak{n}}
$$

is defined to be the composition ${ }^{\mathfrak{c}} \mathbf{S}_{d} \stackrel{\tilde{\Delta}^{\mathfrak{c}}}{\longrightarrow}{ }^{\mathfrak{c}} \mathbf{S}_{d} \otimes \mathbf{S}_{\mathfrak{n}} \stackrel{1 \otimes \chi}{\longrightarrow}{ }^{\mathfrak{c}} \mathbf{S}_{d-\mathfrak{n}} \otimes \mathcal{A}={ }^{\mathfrak{c}} \mathbf{S}_{d-\mathfrak{n}}$, where $\chi\left(\eta_{A}\right)=$ $\operatorname{det}(A)$ for any $A \in{ }^{\mathfrak{c}} \Xi$ and $\eta_{A}$ is the characteristic function on the orbit corresponding the matrix $A$. This is analogous to the transfer map $\phi_{d, d-\mathfrak{n}}^{\jmath}: \mathbf{S}_{d}^{J} \rightarrow \mathbf{S}_{d-\mathfrak{n}}^{\jmath}$ defined in [FL15, §3.6] in the finite type $B$ setting. By Proposition A.3.3, we have the following proposition.

Proposition A.3.5. The following diagram is commutative:

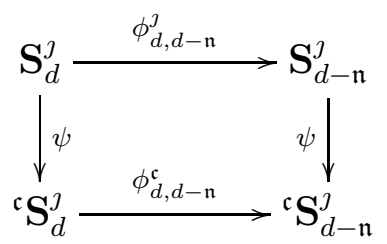


Finally, we address the $\imath$-version. Recall that $\eta=\mathfrak{n}-1=2 r$. Let

$$
X_{\mathfrak{c}}^{\imath}=\left\{0=V_{0} \subseteq V_{1} \subseteq \ldots \subseteq V_{\eta}=\mathbb{F}_{q}^{2 d} \mid V_{\eta-i}=V_{i}^{\perp}\right\} .
$$

The convolution algebra on $X_{\mathfrak{c}}^{\imath} \times X_{\mathfrak{c}}^{\imath}$ is denoted by ${ }^{\mathfrak{c}} \mathbf{S}_{d}^{\imath}$. We shall naturally embed $X_{\mathfrak{c}}^{\imath}$ into $X_{\mathfrak{c}}$ by sending a $\eta$-step flag in $X_{\mathfrak{c}}^{\imath}$ as above to an $\mathfrak{n}$-step flag

$$
0=V_{0} \subseteq V_{1} \subseteq \ldots \subseteq V_{r} \subseteq V_{r} \subseteq \ldots \subseteq V_{\eta}=\mathbb{F}_{q}^{2 d}
$$

(where the maximal isotropic subspace $V_{r}$ in the middle is repeated). Therefore, ${ }^{\mathrm{c}} \mathbf{S}_{d}^{\imath}$ is naturally a subalgebra of ${ }^{\mathfrak{c}} \mathbf{S}_{d}$. Consider the following set

$$
\Xi_{\mathfrak{c}}^{\imath}=\left\{A=\left(a_{i j} \in{ }^{\mathfrak{c}} \Xi \mid a_{r+1, j}=0=a_{i, r+1}, \forall i, j\right\} .\right.
$$

By [BKLW14, Lemma 6.1], we have a natural bijection $\operatorname{Sp}(2 d) \backslash X_{\mathfrak{c}}^{\imath} \times X_{\mathfrak{c}}^{\imath} \leftrightarrow{ }^{\mathfrak{c}} \Xi^{\imath}$, and moreover, $\left\{[A] \mid A \in \Xi_{\mathfrak{c}}^{\imath}\right\}$ forms a basis of ${ }^{\mathfrak{c}} \mathbf{S}_{d}^{\imath}$. Recall a completely analogous subalgebra $\mathbf{S}_{d}^{\imath}$ of $\mathbf{S}_{d}^{\jmath}$ was defined in [BKLW14, §5]. The standard basis of $\mathbf{S}_{d}^{\imath}$ is parametrized by a subset $\Xi^{\imath} \subset \Xi$, and there is a natural bijection $\Xi^{\imath} \longrightarrow \Xi_{\mathfrak{c}}^{\imath}, A \mapsto A-E_{r+1, r+1}$. The following proposition follows by the definition of $\psi$.

Proposition A.3.6. The restriction of $\psi: \mathbf{S}_{d}^{\jmath} \longrightarrow{ }^{\mathrm{c}} \mathbf{S}_{d}^{\jmath}$ induces an algebra isomorphism ${ }^{\mathrm{c}} \mathbf{S}_{d}^{\imath} \simeq \mathbf{S}_{d}^{\imath}$.

Remark A.3.7. It should be clear for the reader that the various canonical bases from finite type $\mathrm{B} / \mathrm{C}$ geometries are compatible under the isomorphism $\psi$. 


\section{List of Notations}

$\mathfrak{a}\{A\}_{d} \quad$ Canonical basis element of $\mathbf{S}_{n, d}$

$\S 2.2, \mathrm{p} 18$

${ }^{a}\{A\} \quad$ Canonical basis element of $\dot{\mathbf{K}}_{n}$

$\S 9.5, \mathrm{p} 89$

$\{A\}_{d} \quad$ Canonical basis element of $\mathbf{S}_{n, d}^{c}$

$\S 4.2$, p 31

$\{A\}$

$\mathfrak{a}[A]$

Stably canonical basis element of $\dot{\mathbf{K}}_{n}^{\mathrm{c}}$

$\S 9.4, \mathrm{p} 88$

$[A]$

$[A]$

$[A]$

$\mathcal{A}$

$\left[\begin{array}{l}a \\ b\end{array}\right]$

$\left[\begin{array}{c}a \\ b\end{array}\right]_{v, v^{\prime}}$

Standard basis element of $\dot{\mathbf{K}}_{n}$

$\S 9.5, \mathrm{p} 89$

Standard basis element of $\mathbf{S}_{n, d}^{\mathrm{c}}$

$\S 4.2$, p 31

Standard basis element of $\mathbf{S}_{n, d}$

$\S 2.2, \mathrm{p} 18$

Standard basis element of $\dot{\mathbf{K}}_{n}^{\mathfrak{c}}$

$\S 9.4, \mathrm{p} 86$

$\S 2.2, \mathrm{p} 17$

Ring of Laurent polynomials $\mathbb{Z}\left[v, v^{-1}\right]$

$\S 2.2, \mathrm{p} 18$

Quantum $v$-binomial coefficient

$\S 9.2, \mathrm{p} 82$

$A_{i, R, t} \quad$ Matrix obtained from $A$ by shifting entries up by one row

$\S 9.2, \mathrm{p} 82$

$\dot{\mathbf{B}}_{n}^{\mathrm{c}} \quad$ Canonical basis of $\dot{\mathbf{U}}_{n}^{\mathrm{c}}$

$\operatorname{co}(A) \quad$ Column sum vector of matrix $A$

$\S 6.4, \mathrm{p} 56$

$\S 2.2, \mathrm{p} 17$

. $\quad$ Bijection $\Xi_{n, d} \rightarrow \Xi_{\breve{n}, d}$ whose inverse is dlt ${ }_{1}$

$\Delta^{\mathfrak{c}} \quad$ Comultiplication $\mathbf{S}_{n, d}^{\mathrm{c}} \rightarrow \mathbf{S}_{n, d^{\prime}}^{\mathfrak{c}} \otimes \mathbf{S}_{n, d^{\prime \prime}}$

$\S 9.1, \mathrm{p} 78$

$\S 5.3, \mathrm{p} 44$

$\Delta_{\mathbf{b}^{\prime}, \mathbf{a}^{\prime}, \mathbf{b}^{\prime \prime}, \mathbf{a}^{\prime \prime}}^{\mathfrak{c}} \quad$ Component of $\Delta^{\mathfrak{c}}$ sending $\mathbf{S}_{n, d}^{\mathfrak{c}}(\mathbf{b}, \mathbf{a})$ to $\mathbf{S}_{n, d}^{\mathfrak{c}}\left(\mathbf{b}^{\prime}, \mathbf{a}^{\prime}\right) \otimes \mathbf{S}_{n, d^{\prime \prime}}\left(\mathbf{b}^{\prime \prime}, \mathbf{a}^{\prime \prime}\right)$

$\S 9.3, \mathrm{p} 86$

$\Delta^{\mathrm{c} \dagger} \quad$ Renormalized raw comultiplication $\mathbf{S}_{n, d}^{\mathfrak{c}} \rightarrow \mathbf{S}_{n, d^{\prime}}^{\mathfrak{c}} \otimes \mathbf{S}_{n, d^{\prime \prime}}$

$\Delta^{\mathfrak{c}} \quad$ Comultiplication $\mathbf{U}_{n, \infty}^{\mathfrak{c}} \longrightarrow \mathbf{U}_{n, \infty}^{\mathfrak{c}} \otimes \mathbf{U}_{n, \infty}$

$\S 5.3, \mathrm{p} 43$

$\S 6.1, \mathrm{p} 51$

$\dot{\Delta}^{\mathfrak{c}} \quad$ Comultiplication of $\dot{\mathbf{K}}_{n}^{\mathfrak{c}}$

$\S 9.6$, p 91

$\dot{\Delta} \quad$ Comultiplication of $\dot{\mathbf{K}}_{n}$

$\S 9.5, \mathrm{p} 89$

$\widetilde{\Delta}^{\mathfrak{c}} \quad$ Raw comultiplication $\mathbf{S}_{n, d}^{\mathfrak{c}} \rightarrow \mathbf{S}_{n, d^{\prime}}^{\mathfrak{c}} \otimes \mathbf{S}_{n, d^{\prime \prime}}$

$\S 5.2, \mathrm{p} 41$

$\operatorname{dep}(A) \quad$ Depth of matrix $A$

$\S 9.1, \mathrm{p} 79$

$\mathrm{dlt}_{i} \quad$ Operation of deleting $k$ th rows/columns for $k \equiv i \bmod n$

$\S 7.3, \mathrm{p} 63$

$E_{\theta}^{i j} \quad n$-periodic centrosymmetric elementary matrix

$E^{i j} \quad n$-periodic elementary matrix

$\mathbf{f}_{A ; d} \quad$ Monomial basis element of $\mathbf{S}_{n, d}^{\mathfrak{c}}$

$\S 4.3, \mathrm{p} 32$

$\S 2.2, \mathrm{p} 18$

$\S 9.1$, p 79

$\mathbf{f}_{A} \quad$ Monomial basis element of $\dot{\mathbf{K}}_{n}^{\mathrm{c}}$

$F \quad$ Field of formal Laurent series $k((\varepsilon))$

$\S 9.4, \mathrm{p} 87$

$\S 2.1, \mathrm{p} 16$

$\mathbf{J}_{0} \quad$ Idempotent of type $\vartheta$ in $\mathbf{S}_{n, d}$

$\S 8.1, \mathrm{p} 69$

$\mathbf{j}_{0} \quad$ Idempotent of type $\vartheta$ in $\mathbf{S}_{n, d}^{\mathfrak{c}}$

$\jmath_{n, d} \quad$ Monomorphism $\mathbf{S}_{n, d}^{\mathfrak{c}} \rightarrow \mathbf{S}_{n, d}$

$\S 8.1, \mathrm{p} 68$

$\jmath_{n} \quad$ Monomorphism $\mathbf{U}_{n}^{\mathfrak{c}} \rightarrow \mathbf{U}_{n}$

$\S 5.3, \mathrm{p} 46$

$\mathbf{J}_{r, 0} \quad$ Idempotent of type $u$ in $\mathbf{S}_{n, d}$

$\S 6.1, \mathrm{p} 51$

$\S 8.4, \mathrm{p} 72$

$\mathbf{j}_{r, 0} \quad$ Idempotent of type $u$ in $\mathbf{S}_{n, d}^{\mathfrak{c}}$

$\S 8.4, \mathrm{p} 72$ 


\begin{tabular}{|c|c|c|}
\hline $\mathbf{J}_{r}$ & Idempotent of type $\imath$ in $\mathbf{S}_{n, d}$ & $\S 7.1, \mathrm{p} 60$ \\
\hline $\mathbf{j}_{r}$ & Idempotent of type $\jmath$ in $\mathbf{S}_{n, d}^{\mathfrak{c}}$ & $\S 7.1, \mathrm{p} 59$ \\
\hline$\dot{\mathbf{K}}_{n}^{\mathfrak{c}}$ & Stabilization algebra of affine type $\mathrm{C}$ & $\S 9.4, \mathrm{p} 86$ \\
\hline$\dot{\mathbf{K}}_{\eta}^{\imath \imath}$ & Stabilization algebra of type $u$ & $\S 10.5, \mathrm{p} 100$ \\
\hline$\dot{\mathbf{K}}_{\mathfrak{n}}^{\imath \jmath}$ & Stabilization algebra of type $\vartheta$ & $\S 10.4, \mathrm{p} 99$ \\
\hline$\dot{\mathbf{K}}_{\mathfrak{n}}^{\jmath \imath}$ & Stabilization algebra of type $n$ & $\S 10.3, \overline{\mathrm{p} 96}$ \\
\hline$\dot{\mathbf{K}}_{n}$ & Stabilization algebra of affine type A & $\S 9.5, \mathrm{p} 89$ \\
\hline$k$ & Finite field of $q$ elements & $\S 2.1, \mathrm{p} 16$ \\
\hline$\Lambda_{n, d}^{\mathfrak{c}}$ & Set of $n$-periodic symmetric tuples in $\mathbb{N}^{\mathbb{Z}}$ of size $d$ & $\S 5.1, \mathrm{p} 40$ \\
\hline$\Lambda_{n, d}$ & Set of $n$-periodic tuples in $\mathbb{N}^{\mathbb{Z}}$ of size $d$ & $\S 2.2, \mathrm{p} 17$ \\
\hline$\leqslant$ alg & An algebraic partial order on $\Theta_{n, d}$ & $\S 2.2, \overline{\mathrm{p} 19}$ \\
\hline & Semi-monomial basis element of $\dot{\mathbf{K}}_{n}^{\mathfrak{c}}$ & $\S 9.4, \mathrm{p} 87$ \\
\hline$(\mathbf{c}, \mathbf{b}) \models \mathbf{a}$ & $a_{i}=c_{i}+b_{i}+b_{-i}$ for all $i$ & $\S 9.3, \mathrm{p} 86$ \\
\hline $\mathbf{b} \models \mathbf{a}$ & $a_{i}=b_{i}+b_{-i}+\delta_{i, n}+\delta_{i, r+1}$ for all $1 \leqslant i \leqslant n$ & $\S 5.4, \mathrm{p} 47$ \\
\hline$\breve{n}$ & $=2 \breve{r}+2=n+2$ & $\S 9.1, \mathrm{p} 78$ \\
\hline$\eta$ & $=\mathfrak{n}-1=n-2=2 r$ & $\S 8.0, \mathrm{p} 68$ \\
\hline $\mathfrak{n}$ & $=n-1=2 r+1$ & $\S 7.0, \overline{\mathrm{p} 59}$ \\
\hline $\mathfrak{o}$ & Ring of formal power series $k[[\varepsilon]]$ & $\S 2.1, \mathrm{p} 16$ \\
\hline${ }_{p} A$ & Matrix $A+p I_{n}$ & $\S 9.2, \overline{\mathrm{p} 84}$ \\
\hline$\ddot{p} A$ & Matrix $A+p\left(I_{\breve{n}}-E_{\theta, \breve{n}}^{1,1}\right)$ & $\S 9.2, \mathrm{p} 82$ \\
\hline$\phi_{d, d-n}^{\mathfrak{c}}$ & Transfer map between Schur algebras of affine type C & $\S 6.1, \mathrm{p} 50$ \\
\hline$\phi_{d, d-n}$ & Transfer map between Lusztig algebras of affine type A & $\S 2.3, \mathrm{p} 21$ \\
\hline$\prod_{n, d}$ & Set of certain $(0,1)$-matrices of affine type $\mathrm{C}$ & $\S 3.2, \overline{\mathrm{p} 25}$ \\
\hline$\Psi_{\eta, d}^{2 n}$ & Surjection $\dot{\mathbf{K}}_{\eta}^{\imath \imath} \rightarrow \mathbf{S}_{\eta, d}^{\imath \imath}$ & $\S 10.5, \mathrm{p} 100$ \\
\hline$\Psi_{\mathfrak{n}, d}^{\imath \jmath}$ & Surjection $\dot{\mathbf{K}}_{\mathfrak{n}}^{\imath \jmath} \rightarrow \mathbf{S}_{\mathfrak{n}, d}^{i \jmath}$ & $\S 10.4, \mathrm{p} 99$ \\
\hline$\Psi_{\mathfrak{n}, d}^{\jmath \imath}$ & Surjection $\dot{\mathbf{K}}_{\mathfrak{n}}^{\jmath \imath} \rightarrow \mathbf{S}_{\mathfrak{n}, d}^{\jmath \imath}$ & $\S 10.3, \mathrm{p} 98$ \\
\hline$\Psi_{n, d}$ & Surjection $\dot{\mathbf{K}}_{n}^{\mathfrak{c}} \rightarrow \mathbf{S}_{n, d}^{\mathfrak{c}}$ & $\S 9.7, \mathrm{p} 92$ \\
\hline$\breve{r}$ & $=r+1$ & $\S 9.1, \overline{\mathrm{p} 78}$ \\
\hline $\mathscr{R}$ & Algebra $\mathbb{Q}(v)\left[v^{\prime}, v^{\prime-1}\right]$ with bar involution & $\S 9.2, \mathrm{p} 82$ \\
\hline$\rho$ & Algebra imbedding $\mathbf{S}_{n, d}^{\mathfrak{c}} \rightarrow \mathbf{S}_{\breve{n}, d}^{\mathfrak{c}}$ & $\S 9.1, \mathrm{p} 78$ \\
\hline $\operatorname{ro}(A)$ & Row sum vector of matrix $A$ & $\S 2.2, \mathrm{p} 17$ \\
\hline $\mathbf{S}_{n, d}^{\mathfrak{c}}(\mathbf{b}, \mathbf{a})$ & Subspace of $\mathbf{S}_{n, d}^{\mathfrak{c}}$ of shape $(\mathbf{b}, \mathbf{a})$ & $\S 5.3, \underline{\mathrm{p} 43}$ \\
\hline $\begin{array}{l}\mathbf{S}_{n, d}^{\mathfrak{c}} \\
. .\end{array}$ & Schur algebra of affine type C & $\S 4.2, \mathrm{p} 31$ \\
\hline $\mathbf{S}_{n, d}^{\mathfrak{c}}$ & Subalgebra of $\mathbf{S}_{\breve{n}, d}^{\mathfrak{c}}$ isomorphic to $\mathbf{S}_{n, d}^{\mathfrak{c}}$ & $\S 9.1, \mathrm{p} 78$ \\
\hline $\mathbf{S}_{\eta, d}^{n \imath}$ & Schur algebra of type $n$ & $\S 8.4, \mathrm{p} 72$ \\
\hline $\mathbf{S}_{\mathfrak{n}, d}^{\imath j}$ & Schur algebra of type $\vartheta$ & $\S 8.1, \overline{\mathrm{p} 68}$ \\
\hline $\mathbf{S}_{\mathfrak{n}, d}^{j i}$ & Schur algebra of type $\imath$ & $\S 7.1, \mathrm{p} 59$ \\
\hline $\mathbf{S}_{n, d}(\mathbf{b}, \mathbf{a})$ & Subspace of $\mathbf{S}_{n, d}$ of shape $(\mathbf{b}, \mathbf{a})$ & $\S 5.3, \mathrm{p} 43$ \\
\hline $\mathbf{S}_{n, d}$ & Schur algebra of affine type $\mathrm{A}$ & $\S 2.2, \mathrm{p} 18$ \\
\hline${ }^{c} \Sigma_{d}$ & Set of permutation matrices of affine type $\mathrm{C}$ & $\S 3.1, \mathrm{p} 24$ \\
\hline$\Sigma_{d}$ & Set of permutation matrices of affine type B & $\S 4.2, \mathrm{p} 30$ \\
\hline$\stackrel{1}{\subset}, \stackrel{1}{\supset}$ & Inclusions of codimension 1 & $\S 5.1, \mathrm{p} 39$ \\
\hline$\Theta_{n, d}^{a p}$ & Set of all aperiodic matrices in $\Theta_{n, d}$ & $\S 2.2, \mathrm{p} 18$ \\
\hline
\end{tabular}


$\Theta_{\eta, d}^{\imath \imath} \quad$ Set of matrices in $\Theta_{n, d}$ whose 0 th $/ r+1$ th rows/columns are zero

$\S 8.4, \mathrm{p} 72$

$\Theta_{\mathfrak{n}, d}^{\imath \jmath}$

Set of matrices in $\Theta_{n, d}$ whose 0th row/column are zero

$\Theta_{\mathfrak{n}, d}^{j i}$

Set of matrices in $\Theta_{n, d}$ whose $r+1$ st row/column are zero

$\S 8.1, \mathrm{p} 69$

$\Theta_{n, d}$

Set of $n$-periodic $\mathbb{Z} \times \mathbb{Z} \mathbb{N}$-matrices of size $d$

$\S 7.1, \mathrm{p} 60$

$\S 2.2, \mathrm{p} 17$

$\widetilde{\Theta}_{n}$

Enlarged matrix set from $\Theta_{n}$ by allowing negative diagonal

$\S 9.5, \mathrm{p} 89$

Idempotented form of $\mathbf{U}_{n}^{\mathfrak{c}}$

$\S 6.2, \mathrm{p} 53$

Lusztig algebra of affine type $\mathrm{C}$

$\S 5.1, \mathrm{p} 39$

$\mathbf{U}_{n, d}^{\mathfrak{c}}$

$\mathbf{U}_{n, \infty}^{\mathfrak{c}, \infty}$

Projective limit of Lusztig algebras of affine type $\mathrm{C}$

$\mathbf{U}_{n}^{\mathfrak{c}}$

Subalgebra of $\mathbf{U}_{n, \infty}^{\mathfrak{c}}$ generated by the Chevalley generators

$\S 6.1, \mathrm{p} 50$

$\dot{\mathbf{U}}_{n} \quad$ Idempotented form of $\mathbf{U}_{n}$

$\S 6.1, \mathrm{p} 51$

$\dot{\mathrm{U}}\left(\widehat{\mathfrak{s l}}_{n}\right)$

$\mathbf{U}_{\eta, d}^{\imath \imath}$

Idempotented quantum affine $\mathfrak{s l}_{n}$

$\S 2.3, \mathrm{p} 22$

$\S 2.3, \mathrm{p} 22$

$\S 8.4, \mathrm{p} 72$

$\mathbf{U}_{\mathfrak{n}, \infty}^{\imath \jmath}$

$\mathbf{U}_{\mathfrak{n}}^{\imath j}$

$\mathbf{U}_{\mathfrak{n}, d}^{\jmath \imath}$

$\mathbf{U}_{\mathfrak{n}, \infty}^{\jmath l}$

$\mathbf{U}_{\mathfrak{n}}^{\jmath 2}$

Lusztig algebra of type $u$

$\S 8.3, \mathrm{p} 71$

Projective limit of Lusztig algebras of type $\vartheta$

Subalgebra of $\mathbf{U}_{\mathfrak{n}, \infty}^{\imath \jmath}$ generated by the Chevalley generators

$\S 8.3, \mathrm{p} 71$

Lusztig algebra of type $n$

Projective limit of Lusztig algebras of type $\imath$

$\S 7.1, \mathrm{p} 60$

$\S 7.4, \mathrm{p} 65$

Subalgebra of $\mathbf{U}_{\mathfrak{n}, \infty}^{\jmath}$ generated by the Chevalley generators

$\S 7.4, \mathrm{p} 65$

$\mathbf{U}_{n, d} \quad$ Lusztig algebra of affine type A

$\mathbf{U}_{n, \infty}$

$\mathbf{U}_{n}$

Projective limit of Lusztig algebras of affine type A

$\mathbf{U}\left(\widehat{\mathfrak{s l}}_{n}\right)$

Subalgebra of $\mathbf{U}_{n, \infty}$ generated by the Chevalley generators

$\S 2.2, \mathrm{p} 18$

$\S 2.3, \mathrm{p} 21$

$\S 2.3, \mathrm{p} 21$

Quantum affine $\mathfrak{s l}_{n}$

$\mathcal{X}_{n, d}^{\mathfrak{c}}(\mathbf{a})$

Affine type $\mathrm{C} n$-step partial flags of shape a

$\mathcal{X}_{n, d}^{\mathfrak{c}}$

Lattice model of $n$-step partial flags of affine type C

$\S 2.3, \mathrm{p} 22$

$\S 5.3, \mathrm{p} 43$

$\S 3.2, \mathrm{p} 25$

$\mathcal{X}_{n, d}(\mathbf{a})$

Affine type A $n$-step partial flags of shape a

$\mathcal{X}_{n, d}$

$\Xi_{n, d}^{a p}$

Lattice model of $n$-step partial flags of affine type A

$\S 5.3, \mathrm{p} 43$

$\S 2.2, \mathrm{p} 17$

Set of aperiodic matrices in $\Xi_{n, d}$

Set of $n$-periodic centrosymmetric $\mathbb{N}$-matrices of size $d$ of type $\mathrm{B}$

$\S 5.4, \mathrm{p} 47$

$\S 4.2, \mathrm{p} 29$

Set of $n$-periodic centrosymmetric $\mathbb{N}$-matrices of size $d$ of type $\mathrm{C}$

$\S 3.2, \mathrm{p} 26$

${ }^{\mathfrak{c}} \Xi_{n, d}$

$\Xi_{\eta, d}^{n,}$

$\Xi_{\mathfrak{n}, d}^{\imath j}$

Set of matrices in $\Xi_{n, d}$ whose 0 th $/ r+1$ th rows/columns are zero

$\S 8.4, \mathrm{p} 72$

Set of matrices in $\Xi_{n, d}$ whose 0 th row/column are zero

$\S 8.1, \mathrm{p} 68$

Set of matrices in $\Xi_{n, d}$ whose $r+1$ st row/column are zero

$\S 7.1, \mathrm{p} 59$

$\Xi_{\mathfrak{n}, d}^{\jmath n}$

$\Xi_{n, d}(A)$

$\widetilde{\Xi}_{n}^{a p}$

$\stackrel{\Xi}{\Xi}_{n, d}^{n}$

Set of matrices $B$ in $\Theta_{n, d}$ such that $\operatorname{co}(B) \models \operatorname{co}(A)$

$\S 5.4, \mathrm{p} 47$

Set of aperiodic matrices in $\widetilde{\Xi}_{n}$

$\S 6.2, \mathrm{p} 53$

Set of matrices of size $d$ in $\widetilde{\Xi}_{n}$

$\S 6.2, \mathrm{p} 53$

Enlarged matrix set from $\Xi_{n}$ by allowing negative diagonal

$\S 6.2, \mathrm{p} 53$

Lattice model of complete flags of affine type A

$\S 2.1, \mathrm{p} 16$

Lattice model of complete flags of affine type $\mathrm{C}$

$\S 3.1, \mathrm{p} 24$

Aperiodic monomial in $\mathbf{U}_{n, d}$ associated to a matrix $A$

$\S 2.2, \mathrm{p} 20$

Aperiodic monomial in $\mathbf{U}_{n, d}^{\mathfrak{c}}$ associated to a matrix $A$

$\S 5.4, \mathrm{p} 47$

Set of $n$-periodic tuples in $\mathbb{Z}^{\mathbb{Z}}$

$\S 2.3, \mathrm{p} 21$

Set of $n$-periodic symmetric tuples in $\mathbb{Z}^{\mathbb{Z}}$

$\S 6.2, \mathrm{p} 52$

$\mathbb{Z}_{n}$ 


\section{REFERENCES}

[BBD82] A.A. Beilinson, J. Bernstein and P. Deligne, Faisceaux pervers, Astérisque 100 (1982).

[BLM90] A. Beilinson, G. Lusztig and R. McPherson, A geometric setting for the quantum deformation of $G L_{n}$, Duke Math. J., 61 (1990), 655-677.

[Bao16] H. Bao, Kazhdan-Lusztig theory of super type D and quantum symmetric pairs, arXiv:1603.05105.

[BKLW14] H. Bao, J. Kujawa, Y. Li, and W. Wang, Geometric Schur duality of classical type, Transform. Groups (to appear), arXiv:1404.4000v3.

[BLW14] H. Bao, Y. Li, and W. Wang, A geometric setting for the coideal algebra $\dot{\mathbf{U}}^{\imath}$ and compatibility of canonical bases, Appendix to BKLW14, 15pp. Transform. Groups (to appear).

[Br03] T. Braden, Hyperbolic localization of intersection cohomology, Transform. Groups 8 (2003), 209216.

[BK15] M. Balagovic and S. Kolb, Universal K-matrix for quantum symmetric pairs, arXiv:1507.06276v2, J. Reine Angew. Math. (to appear), DOI 10.1515/crelle-2016-0012.

[BW13] H. Bao and W. Wang, A new approach to Kazhdan-Lusztig theory of type B via quantum symmetric pairs, arXiv:1310.0103v2.

[BW16] H. Bao and W. Wang, Canonical bases arising from quantum symmetric pairs, arXiv:1610.09271.

[CG97] N. Chriss and V. Ginzburg, Representation Theory and Complex Geometry, Birkhäuser, Boston (1997).

[CP96] V. Chari, A. Pressley, Quantum affine algebras and affine Hecke algebras, Pacific J. Math. 174 (1996), 295-326.

[DD91] R. Dipper and S. Donkin, Quantum $G L_{n}$, Proc. London Math. Soc. 63 (1991), 165-211.

[DD05] B. Deng and J. Du, Monomial bases for quantum affine sl ${ }_{n}$, Adv. in Math. 191 (2005), 276-304.

[DDF12] B. Deng, J. Du and Q. Fu, A double Hall algebra approach to affine quantum Schur-Weyl theory. London Mathematical Society Lecture Note Series, 401. Cambridge University Press, Cambridge, 2012.

[DDPW08] B. Deng, J. Du, B. Parshall and J. Wang, Finite dimensional algebras and quantum groups, Mathematical Surveys and Monographs 150, Amer. Math. Soc., Providence, RI, 2008.

[DF13] J. Du and Q. Fu, Quantum affine $\mathfrak{g l}_{n}$ via Hecke algebras, Adv. in Math. 282 (2015), 23-46.

[DF14] J. Du and Q. Fu, The integral quantum loop algebra of $\mathfrak{g l}_{n}$, arXiv:1404.5679.

[DJ89] R. Dipper and G. James, The q-Schur algebra, Proc. London Math. Soc. 59 (1989), 23-50.

[DJ91] R. Dipper and G. James, q-tensor space and $q$-Weyl modules, Trans. Amer. Math. Soc. 327 (1991), 251-282.

[Dr86] V. Drinfeld, Quantum groups, Proc. Int. Congr. Math. Berkeley 1986, vol. 1, Amer. Math. Soc. $1988,798-820$.

[Du92] J. Du, Kazhdan-Lusztig bases and isomorphism theorems for q-Schur algebras, In: KazhdanLusztig theory and related topics (Chicago, IL, 1989), 121-140, Contemp. Math., 139, Amer. Math. Soc., Providence, RI, 1992.

[ES13] M. Ehrig and C. Stroppel, Nazarov-Wenzl algebras, coideal subalgebras and categorified skew Howe duality, arXiv:1310.1972,

[FLLLW] Z. Fan, C. Lai, Y. Li, L. Luo and W. Wang, Affine Hecke algebras and quantum symmetric pairs, arXiv:1609.06199.

[FL14] Z. Fan and Y. Li, Geometric Schur duality of classical type, II, Trans. Amer. Math. Soc., Series B2 (2015), 51-92.

[FL15] Z. Fan and Y. Li, Positivity of canonical basis under comultiplication, arXiv:1511.02434.

[FL17] Z. Fan and Y. Li, Affine flag varieties and quantum symmetric pairs, II. Multiplication formula, arXiv:1701.06348

[G97] R. Green, Hyperoctahedral Schur algebras, J. Algebra 192 (1997), 418-438.

[G99] R. Green, The affine q-Schur algebra, J. Algebra 215 (1999), 379-411.

[GL92] I. Grojnowski and G. Lusztig, On bases of irreducible representations of quantum $G L_{n}$. In: Kazhdan-Lusztig theory and related topics (Chicago, IL, 1989), 167-174, Contemp. Math. 139, Amer. Math. Soc., Providence, RI, 1992. 
[GV93] V. Ginzburg and E. Vasserot, Langlands reciprocity for affine quantum groups of type $A_{n}$, Internat. Math. Res. Notices 3 (1993), 67-85.

[GRV93] V. Ginzburg, N. Reshetikhin and E. Vasserot, Quantum groups and flag varieties, Mathematical aspects of conformal and topological field theories and quantum groups (South Hadley, MA, 1992), 101-130, Contemp. Math., 175, Amer. Math. Soc., Providence, RI, 1994

[H99] R. Howe, Affine-like Hecke algebras and p-adic representation theory. in Iwahori-Hecke algebras and their representation theory (Martina-Franca, 1999), 27-69, Lecture Notes in Math., 1804, Springer, Berlin, 2002.

[Iw64] N. Iwahori, On the structure of a Hecke ring of a Chevalley group over a finite field, J. Fac. Sci. Univ. Tokyo Sect. I 10 (1964), 215-236.

[IM65] N. Iwahori and H. Matsumoto, On some Bruhat decomposition and the structure of the Hecke rings of p-adic Chevalley groups, Publications math. I.H.E.S. 25 (1965), 5-48.

[Jim86] M. Jimbo, A q-analogue of $U(\mathfrak{g l}(N+1))$, Hecke algebra, and the Yang-Baxter equation, Lett. Math. Phys. 11 (1986), 247-252.

[K91] M. Kashiwara, On crystal bases of the Q-analogue of universal enveloping algebras, Duke Math. J. 63 (1991), 456-516.

[K94] M. Kashiwara, Crystal bases of modified quantized enveloping algebra, Duke Math. J. 73 (1994) 383-413.

[Ko14] S. Kolb, Quantum symmetric Kac-Moody pairs, Adv. in Math. 267 (2014), 395-469.

[KL79] D. Kazhdan and G. Lusztig, Representations of Coxeter groups and Hecke algebras, Invent. Math. 53 (1979), 165-184.

[Le99] G. Letzter, Symmetric pairs for quantized enveloping algebras, J. Algebra 220 (1999), 729-767.

[Le02] G. Letzter, Coideal subalgebras and quantum symmetric pairs, New directions in Hopf algebras (Cambridge), MSRI publications, vol. 43, Cambridge Univ. Press, 2002, pp. 117-166.

[LL15] C. Lai and L. Luo, An elementary construction of monomial bases of quantum affine $\mathfrak{g l}_{n}$, J. London Math. Soc. (to appear). arXiv:1506.07263.

[Lu90] G. Lusztig, Canonical bases arising from quantized enveloping algebras, J. Amer. Math. Soc. 3 (1990), 447-498.

[Lu93] G. Lusztig, Introduction to quantum groups, Progress in Mathematics 10, Birkhäuser Boston, Inc., Boston, MA, 1993.

[Lu97] G. Lusztig, Cells in affine Weyl groups and tensor categories, Adv. in Math. 129 (1997), 85-98.

[Lu99] G. Lusztig, Aperiodicity in quantum affine $\mathfrak{g l}_{n}$, Asian J. Math. 3 (1999), 147-177.

[Lu00] G. Lusztig, Transfer maps for quantum affine $\mathfrak{s l}_{n}$, in Representations and quantizations, (ed. J. Wang et. al.), China Higher Education Press and Springer Verlag 2000, 341-356.

[Lu03] G. Lusztig, Hecke algebras with unequal parameters, CRM Monograph Series, 18, Amer. Math. Soc., Providence, RI, 2003.

[LW15] Y. Li and W. Wang, Positivity vs negativity of canonical basis, Proceedings for Lusztig's 70th birthday conference, Bulletin of Institute of Mathematics Academia Sinica (N.S.), to appear (2017). arXiv:1501.00688v4

[Mc12] K. McGerty, On the geometric realization of the inner product and canonical basis for quantum affine $\mathfrak{s l}_{n}$, Alg. and Number Theory 6 (2012), 1097-1131.

[N96] M. Noumi, Macdonalds symmetric polynomials as zonal spherical functions on some quantum homogeneous spaces, Adv. in Math. 123 (1996), 16-77.

[OV] A. Onishchik and E. Vinberg, Lie groups and algebraic groups, Translated from the Russian by D.A. Leites. Springer Series in Soviet Mathematics. Springer-Verlag, Berlin, 1990.

[P09] G. Pouchin, A geometric Schur-Weyl duality for quotients of affine Hecke algebras, J. Algebra 321 (2009), 230-247.

[R90] C. Ringel, Hall algebras and quantum groups, Invent. Math. 101 (1990), 583-592.

[Sa99] D. Sage, The geometry of fixed point varieties of affine flag manifolds, Trans. Amer. Math. Soc., 352 (1999), 2087-2119.

[Sch06] O. Schiffmann, Lectures on Hall algebras, arXiv:math/0611617. 
[SV00] O. Schiffmann and E. Vasserot, Geometric construction of the global base of the quantum modified algebra of $\hat{\mathfrak{g l}}_{n}$, Transform. Groups 5 (2000), 351-360.

[VV99] M. Varagnolo and E. Vasserot, On the decomposition matrices of the quantized Schur algebra, Duke Math. J. 100 (1999), 267-297.

School of science, Harbin Engineering University, Harbin, China 150001

E-mail address: fanz@math.ksu.edu (Fan)

Department of Mathematics, University of Virginia, Charlottesville, VA 22904

E-mail address: cl8ah@virginia.edu (Lai)

Department of Mathematics, University at Buffalo, SUNy, Buffalo, Ny 14260

E-mail address: yiqiang@buffalo.edu (Li)

Department of mathematics, East China Normal University, Shanghai, China 200241

E-mail address: lluo@math.ecnu.edu.cn (Luo), ww9c@virginia.edu (Wang) 